Daniel Makoto Tokunaga

Local Pose Estimation of Feature Points for Object Based Augmented Reality 
Daniel Makoto Tokunaga

\section{Local Pose Estimation of Feature Points for Object Based Augmented Reality}

Tese apresentada à Escola Politécnica da Universidade de São Paulo para obtenção do Título de Doutor em Ciências 
Daniel Makoto Tokunaga

\section{Local Pose Estimation of Feature Points for Object Based Augmented Reality}

Tese apresentada à Escola Politécnica da

Universidade de São Paulo para obtenção do Título de Doutor em Ciências

Área de Concentração:

Engenharia de Computação

Orientador:

Prof. Livre-Docente Romero Tori 
Este exemplar foi revisado e corrigido em relação à versão original, sob responsabilidade única do autor e com a anuência de seu orientador.

São Paulo, de de

Assinatura do autor:

Assinatura do orientador:

Catalogação-na-publicação

Tokunaga, Daniel Makoto

Local Pose Estimation of Feature Points for Object Based Augmented Reality / D. M. Tokunaga -- versão corr. -- São Paulo, 2016. $170 \mathrm{p}$.

Tese (Doutorado) - Escola Politécnica da Universidade de São Paulo. Departamento de Engenharia de Computação e Sistemas Digitais.

1.Realidade virtual 2.Computação gráfica 3.Processamento de imagens 4.Realidade Aumentada 5.Detecção de Poses I.Universidade de São Paulo. Escola Politécnica. Departamento de Engenharia de Computação e Sistemas Digitais II.t. 
For who those keep searching, researching and re-searching 


\section{Acknowledgements}

Initially, I wish to make clear that this work is a result of inputs which I received from several people that I have met during my $\mathrm{PhD}$ program. Thus, I will sample some here, but making sure that I should acknowledge a lot, lot, ton more of people.

First, my family, for supporting my $\mathrm{PhD}$ period.

I want also to acknowledge my PhD adviser, here in Escola Politécnica of USP, Romero Tori; for making this work possible. As well as, my adviser of the visiting period at MIT Media Lab, Ramesh Raskar; for the great experience, knowledge and chances given during my visiting period.

Also, special acknowledgement for Munehiko Sato, Dan Raviv and Victor Pamplona; for the advises given during the visiting period, which deeply influenced this work.

Now, listing other names; Cléber G. Corrêa, Mariza U. Leone, Ricardo Nakamura, João Bernardes, Fatima Nunes, Silvio R.R. Sanches, Valdinei Silva, Fernando Y. Obana; Achuta Kadanbi, Ayush Bandari, Refael Whyte, Hayato Ikoma, Chunglin Wen, Barmak Hesht; you are at middle; Micha Feigin, Moshe Ben-Ezra, Hisham Bedri, Belen Masia, Diego Gutierrez, John Werner; Tiago Lucena, Carolina Brum, Ruy de Deus, Fêrnanda Cimini; and PhD course teachers, Claudio S. Pinhanez, Carlos Humes Jr, Sérgio S. Furuie, Carlos H. Morimoto, Junior Barrera and Anna H. R. Costa.

For who those I could not list the names here, due to lack of space; I wish to remember that your input was also crucial for this work. Thus, I want to acknowledge for what you had done for me.... or at least, had not done to me.

At very last, I want to acknowledge you, reader of this work. For using your time to read this work (for whatever the reason). And hope that this turns to some kind of input for you too. 
... Now I do not know whether it was then I dreamt I was a butterfly, or whether I am now a butterfly dreaming I am a man... (Zhuangzi, "The Butterfly Dream", translation from "The Three Religions of China: Lectures Delivered" at Oxford (1913) by William Edward Soothill, p. 75) 


\section{Abstract}

Usage of real objects as links between real and virtual information is one key aspect in augmented reality. A central issue to achieve this link is the estimation of the visuospatial information of the observed object, or in other words, estimating the object pose. Different objects can have different behaviors when used for interaction. This not only encompasses changes in position, but also folding or deformations. Traditional researches in the area solve those pose estimation problems using different approaches, depending on the type of the object. Additionally, some researches are based only on positional information of observed feature points, simplifying the object information. In this work, we explore the pose estimation of different objects by gathering more information from the observed feature points, and obtaining the local poses of such points, which are not explored in other researches. We apply this local pose estimation idea in two different capturing scenarios, reaching two novel approaches of pose estimation: one based on RGB-D cameras, and another based on RGB and machine learning methods. In the RGB-D based approach, we use the feature point orientation and near surface to obtain its normal; then, find the local 6 degrees-of-freedom (DoF) pose. This approach gives us not only the rigid object pose, but also the approximated pose of deformed objects. On the other hand, our RGB based approach explores machine learning with local appearance changes. Unlike other RGB based works, we replace the complex non-linear systems solvers with a fast and robust method, reaching local rotation of the observed feature points, as well as, full 6 DoF rigid object pose with dramatically lower real-time calculation demands. Both approaches show us that gathering local poses can bring information for the pose estimation of different types of objects.

Keywords: Augmented Reality. Computer Vision. Pose Estimation. Local Pose. 


\section{Resumo}

O uso de objetos reais como meio de conexão entre informações reais e virtuais é um aspecto chave dentro da realidade aumentada. Uma questão central para tal conexão é a estimativa de informações visuo-espaciais do objeto, ou em outras palavras, a detecção da pose do objeto. Diferentes objetos podem ter diferentes comportamentos quando utilizados em interações. Não somente incluindo a mudança de posição, mas também sendo dobradas ou deformadas. Pesquisas tradicionais solucionam tais problemas de detecção usando diferentes abordagens, dependendo do tipo de objeto. Adicionalmente, algumas pesquisas se baseiam somente na informação posicional dos pontos de interesse, simplificando a informação do objeto. Neste trabalho, a detecção de pose de diferente objetos é explorada coletando-se mais informações dos pontos de interesse observados e, por sua vez, obtendo as poses locais de tais pontos, poses que não são exploradas em outras pesquisas. Este conceito da deteç̧ão de pose locais é aplicada em dois ambientes de capturas, estendendo-se em duas abordagens inovadoras: uma baseada em câmeras RGB-D, e outra baseada em câmeras RGB e métodos de aprendizado de maquinas. Na abordagem baseada em RGB-D, a orientação e superfície ao redor do ponto de interesse são utilizadas para obter a normal do ponto. Através de tais informações a pose local é obtida. Esta abordagem não só permite a obtenção de poses de objetos rígidos, mas também a pose aproximada de objetos deformáveis. Por outro lado, a abordagem baseada em RGB explora o aprendizado de máquina aplicado em alterações das aparências locais. Diferentemente de outros trabalhos baseados em câmeras RGB, esta abordagem substitui solucionadores não lineares complexos com um método rápido e robusto, permitindo a obtenção de rotações locais dos pontos de interesse, assim como, a pose completa (com 6 graus-de-liberdade) de objetos rígidos, com uma demanda computacional muito menor para cálculos em tempo-real. Ambas as abordagens mostram que a coleta de poses locais podem gerar informações para a detecção de poses de diferentes tipos de objetos.

Palavras-chave: Realidade Aumentada. Visão Computacional. Detecção de Poses. Poses Locais. 


\section{List of Figures}

Figure 1 - Results of our work applied in augmented reality applications scenarios. First row: examples of pose estimation applied in different objects; second row: concept images for applications in AR. First column: usage of real object as light source; second and third: usage of object deformation as interaction method for AR games; forth column: information visualization, extending real object visual information. . . . . . . . .

Figure 2 - Information loss in different cases. Conventional methods simplify the object full information $2 \mathrm{a}$ to simple points $2 \mathrm{~b}$. However, more information can be gathered, such as local surface normals $2 \mathrm{c}$ or local appearance $2 \mathrm{~d}$. 2

Figure 3 - Apparatus used in this work. . . . . . . . . . . . 6

Figure 4 - Examples of possible deformations of the same planar object. . . . . . . 10

Figure 5 - Possible deformation estimation based on points. First row: based on point positions; second row: based on local transformations. . . . . . . 11

Figure 6 - Local and global information differences. Feature point $\mathbf{p}_{i}$, has as local information of normal $\vec{n}_{i}$, orientation $\vec{o}_{i}$ and scale $s_{i}$, represented in red color. Other point information $\mathbf{p}_{j}$, normal $\vec{n}_{j}$, orientations $\vec{o}_{j}$ and scales $s_{j}$ are considered global information to $\mathbf{p}_{i}$, represented in black color. . 14

Figure 7 - ASIFT feature points process extraction and matching. . . . . . . . 17

Figure 8 - Definition1, 2-point constrain. . . . . . . . . . . . 25

Figure 9 - The 4 different solutions for the equation (3.4). All the 4 triangles, $\left(\mathbf{p}_{i}, \mathbf{p}_{j}, \mathbf{p}_{k}\right),\left(\mathbf{p}_{i}^{\prime}, \mathbf{p}_{j}, \mathbf{p}_{k}\right),\left(\mathbf{p}_{i}, \mathbf{p}_{j}^{\prime}, \mathbf{p}_{k}\right),\left(\mathbf{p}_{i}, \mathbf{p}_{j}, \mathbf{p}_{k}^{\prime}\right)$, can be solutions. . . . 26

Figure 10 - Locally planar surface assumption. . . . . . . . . . . . . . 47

Figure 11 - texture mapping function, $f_{o b j}\left(T_{t e x}\right)$, illustration. Using this function and its inverse, it is possible to convert the local planar transformation to the original object space and vice-versa. . . . . . . . . . . . . . 47

Figure 12 - Local poses relationship to the object global pose. . . . . . . . . . . . 52

Figure 13 - Appearance changes of the image patch with same location and same orientation. . . . . . . . . . . . . . . . 5 5 . . . . . . . . . . .

Figure 14 - Local RGB-D information to object pose. . . . . . . . . . . . 53

Figure 15 - Information flow. $F_{o b j}$ is the extracted feature points; $T_{l o c a l}$, the local transformation; $R_{\text {local }}$, the local rotation; and $T_{\text {final }}$ is the final object pose. The robust feature point extraction part will be presented in chapter 6; RGB process, in chapter 8; and RGB-D process, in chapter 7. 54

Figure 16 - Information flow of our robust feature points extraction. . . . . . . . . 55

Figure 17 - Perlin noise background used in this work, used to simulate different background conditions. . . . . . . . . . . . . . 56 
Figure 18 - Perlin noise creation process. Consecutive noise adding and resizing creates the sequential sum of noises with different frequencies. . . . . . 57

Figure 19 - Camera pose models. . . . . . . . . . . . . . . . . 58

Figure 20 - Converage area of feature points. Each color area represents the coverage area that a single feature can be observed. If only total area is considered, it can generate several feature points that are visible in the same area and not covering others. Our entropy maximization method tries to maximize the total observation area. . . . . . . . . . . . . . 6 60

Figure 21 - Information flow of our pose estimation approach based on RGB-D information. . . . . . . . . . . . . . . . . 63

Figure 22 - Calculation of $\mathbf{p}_{f t s}$ by $K_{\text {cam }}, \mathbf{p}_{\text {cam }}, \mathbf{p}^{3 D} \ldots \ldots \ldots \ldots$. . . . . . . 64

Figure 23 - Calculation of $\mathbf{p}_{f t s}$ by plane intersection. . . . . . . . . . . . 65

Figure 24 - Calculation of $\vec{v}_{\text {ori }}$. . . . . . . . . . . . . . . . . . 66

Figure 25 - Illustration of the feature points collected information. At right, information of a single feature point; at left, illustration of how are the local information spread over the object surface texture at this point. . . . . 66

Figure 26 - Information flow of our RegressAR pose estimation approach based on RGB information. . . . . . . . . . . . . . . . . . 73

Figure 27 - L2 distances between feature points views with fixed $\theta$, and varying $\phi$, $\psi$ values of camera. Zero valued distance in the graph is the reference view. It is possible to observe the similarity, which appears all across different $\psi$ and $\phi$, making the extension of 2 DoF rotation regression (fixed $\psi$ ) to 3 DoF rotation non-trivial. . . . . . . . . . . . . . . . . . 74

Figure 28 - Camera pose models for regression. Camera model in figure 28a is used in the training part, and figure 28b, for the pose estimation part. . . . 74

Figure 29 - Near feature point image patch extraction for training feature value $\mathbf{v}$ extraction. First row: extraction by image processing; second row: extraction based on coordinate system transformation. . . . . . . . . 77

Figure 30 - Training features values changes across $\theta$ and $\phi$ values, each one with different feature points. Illustrating the changes of pixel color across poses. Moreover, the difference between different feature points. . . . . 78

Figure $31-\psi$ calculation model. . . . . . . . . . . . . . . . . . . 79

Figure $32-z$ calculation model in order to estimate the translation of the object pose. 80 Figure 33 - Results obtained by our background robust feature extraction method. At left, conventional SURF point extraction method applied to the image; at right, our method. . . . . . . . . . . . . . . 85 
Figure 34 - Feature matching results using features extracted by different methods. At first row we show results using synthesized data, and second row, capture image from real environment. First column: feature extracted using conventional SURF methods; second column: our modified ASIFT; third column: our method of entropy maximization method. . . . . . . 86

Figure 35 - First row: feature matching results box plot across view latitude; second row: means plot in same graph. . . . . . . . . . . . . . . . 8 8

Figure 36 - First row: feature matching results box plot across number of extracted feature points use to match feature points at the new frame; second row: means with standard deviation plot in same graph. . . . . . . . . 88

Figure 37 - First row: feature matching results box plot across number of extracted feature points use to match feature points at the new frame with less numbers; second row: means with standard deviation . . . . . . . . . . 89

Figure 38 - Local pose of planar object calculated with our RGB-D local pose method. 90 Figure 39 - Local pose detected X,Y-axis angles between ground truth (for synthesized image) and minimum observation error pose (for real image). First row: poses from synthesized image; second row, from real image. First column: the estimated pose image; second column: graph of the angles.

Figure 40 - Depth maps with noise and result of the local pose estimation based on RGB-D. First row: depth maps with Gaussian noise added with sigma $=[0,15,45,75] \mathrm{mm}$. Second row: respective estimation results of local poses using the depth map above it. . . . . . . . . . . . . . . .

Figure 41 - Box plot of local poses errors across depth map noise and camera orthogonality. First row: errors across depth map noises with random camera orthogonality; second row: errors across camera orthogonality with random depth map noises. First column plots the rotation error in \%; second column, the translation error in \%; third column, $\mathrm{X}$ axis error angle between local and ground truth in rad; fourth column, Y axis error angle between local and ground truth in rad. . . . . . . . . .

Figure 42 - Surface plot of depth map noise and orthogonality interaction effect in local pose errors. . . . . . . . . . . . . . . . . . . . . . . 95

Figure 43 - Local pose of 3D object calculated with our RGB-D local pose method. First row: poses without the object global pose correction done by $f_{o b j}\left(T_{t e x}\right)$; second row: correct local poses to object poses. . . . . . . . . 96

Figure 44 - Local pose of deformable objects. . . . . . . . . . . . . . . . . . 96

Figure 45 - Results of global pose estimation method for planar objects obtained by RANSAC method. Results obtained using real images with camera positions less orthogonal to object plane from images 45a to 45d. There results are obtained by local poses estimated at figure 38 . . . . . . . . 
Figure 46 - Results of global pose estimation method for cylindrical 3D objects. . . 98

Figure 47 - Box plot evaluation of our method and conventional three-points based RANSAC pose. Results across different depth map noise. Upper row: rotation error; second row: translation errors. First column: our local pose approach; second column: three-points based RANSAC. . . . . . . 99

Figure 48 - Box plot evaluation of our method and conventional three-points based RANSAC pose. Results across 2D noise of the feature point position in the image space. Upper row: rotation error; second row: translation errors. First column: our local pose approach; second column: threepoints based RANSAC. . . . . . . . . . . . . . . . . . 100

Figure 49 - Mean process time across added error noise values. . . . . . . . . . . . 101

Figure 50 - Evaluation of our method and three-points based RANSAC, across RANSAC error threshold and depth map noise. Upper row: rotation error. Lower row: process time. . . . . . . . . . . . . . . . . . . 102

Figure 51 - Single feature point pose detection results, in different camera view conditions. The estimated local pose coordinate system is also rendered over the detected position. . . . . . . . . . . . . . . . . 103

Figure 52 - Deformable object pose estimation in several conditions. . . . . . . . . 103

Figure 53 - Deformable object pose estimation, other results. . . . . . . . . . . . . 104

Figure 54 - Trees regression L1 errors. Each row show errors returned by different feature points. First column: errors returned by regression of $\phi$ values; and second, by $\theta$ regression. . . . . . . . . . . . . . . . . . . 105

Figure 55 - Confusion matrix evaluation with 1000 non-trained images; Y axis represents the ground truth value, and $\mathrm{X}$ axis, the predicted value. Also illustrating the errors in $\phi$ regression. . . . . . . . . . . . . . . . . . 105

Figure 56 - Augmented reality results of planar objects obtained by RF-RANSAC. Red line grids represents the entire object pose estimation. First and second rows results are obtained with different cameras with different projection matrix values. . . . . . . . . . . . . . . . . . . . 107

Figure 57 - Approximated pose estimation of 3D rigid objects, obtained by extending our approach for 3D rigid objects. . . . . . . . . . . . . . . . . . 108

Figure 58 - Samples of images with and without background used for the evaluation of the methods. First row, with realistic background; second row, without background.

Figure 59 - Box plot of results evaluation of our approach, compared with EPnP and EPnP-RANSAC by Lepetit, Moreno-Noguer e Fua (2009) First row: evaluation based on images without background images(flat black background); second row: with realistic images as background. First column: rotation error; second:translation errors. . . . . . . . . . . . . . 110 
Figure 60 - Error values across different values of feature points. 60e shows the rotation errors; 60d, the translation error and 60b, the found poses across the evaluation images. . . . . . . . . . . . . . . 111

Figure 61 - Evaluation of translation and rotation errors, and number of founds across all the test set; across different forest trees numbers and different number of forest attached feature points. . . . . . . . . . . . . . . 112

Figure 62 - Deformed object local rotation estimated using our regression forest. . 113 Figure 63 - Light field representations. . . . . . . . . . . . . . . . . . 120

Figure 64 - Articulated objects pose estimation using our local poses. Note that in cases that few feature points are visible because of occlusions, conventional point based methods could not be used, figure 64b. Unlike our local poses cases, figure 64c. . . . . . . . . . . . . . . 126

Figure 65 - Camera parameters estimation impact in AR. Using the table as marker.127 Figure 66 - Usage of actuated objects as markers. Real robot as marker to AR, where the marker itself can sense and actuate over the real world. . . . 130

Figure 67 - View position dependent AR. Display table used as marker. In the same environment, different virtual information (drawn in green) can be rendered according to users positions. Adapting the virtual content to each user. . . . . . . . . . . . . . . . . . . . . . . 130

Figure 68 - Sobel filter (FAUGERAS, 1993) applied to the rendered image of our pose estimation result. The application of other concepts such as presenetd in (TOKUNAGA; NAKAMURA; TORI, 2009), can bring more results of object based AR . . . . . . . . . . . . . . . . . . . . 134 


\section{List of Tables}

Table 1 - Comparison of works based on RGB-D cameras for rigid object pose estimation. . . . . . . . . . . . . . . . . 2 23

Table 2 - Comparison of works based on machine learning for object pose estimation 37

Table 3 - Comparison of works for deformable object pose estimation and its

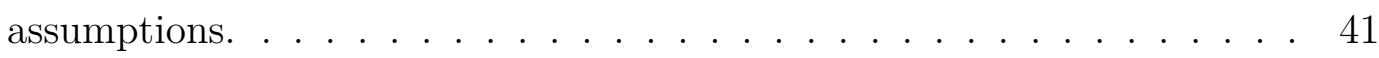

Table 4 - Correlation of object deformation assumptions. . . . . . . . . . 41

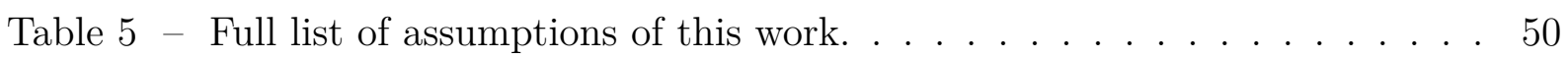

Table 6 - List of expected input information for our approach. . . . . . . . . 50 


\section{List of symbols}

\begin{tabular}{|c|c|}
\hline$I_{\text {color }}$ & captured RGB color image \\
\hline$I_{\text {depth }}$ & captured depth map image \\
\hline$I_{t e x}$ & object surface texture image \\
\hline $\mathbf{K}_{\text {color }}$ & color camera projection matrix \\
\hline $\mathbf{K}_{r}$ & synthesized images render projection matrix \\
\hline$f_{o b j}\left(\mathbf{T}_{t e x}\right)$ & $\begin{array}{l}\text { function to convert transformations between texture space to original } \\
\text { object space }\end{array}$ \\
\hline$\phi$ & zenith angle values in camera models \\
\hline$\theta$ & latitude angle values in camera models \\
\hline$\psi$ & camera axis rotation angles in camera models \\
\hline $\mathrm{p}$ & denotes a points in space \\
\hline$\vec{x}$ & all the variables with upper arrow denotes vectors in space \\
\hline$x^{I}$ & $\begin{array}{l}\text { upper } I \text { denotes that the variable is in the image space (generally a } 2 \mathrm{D} \\
\text { space) }\end{array}$ \\
\hline$x^{w}$ & $\begin{array}{l}\text { upper } w \text { denotes that the variable is in the global world space (generally } \\
\text { a } 3 \mathrm{D} \text { space) }\end{array}$ \\
\hline$x^{o}$ & $\begin{array}{l}\text { upper } o \text { denotes that the variable is in the original object centered } \\
\text { coordinates(generally a } 3 \mathrm{D} \text { space) }\end{array}$ \\
\hline$x^{H}$ & upper $H$ denotes that the variable is in the homogeneous coordinates \\
\hline $\mathbf{T}$ & a transformation matrix \\
\hline $\mathbf{T}_{f t s}$ & feature point local pose transformation matrix \\
\hline $\mathbf{T}_{o b j}$ & object global pose transformation matrix \\
\hline $\mathbf{f}_{o b j}$ & full feature point local information array \\
\hline $\mathbf{p}_{\text {fts }}$ & feature point position in object space \\
\hline$\vec{n}_{f t s}$ & feature point normal in object space position \\
\hline
\end{tabular}


$\vec{o}_{f t s} \quad$ feature point local texture surface orientation in object space position

$d s c_{f t s} \quad$ feature point descriptor

$s_{f t s} \quad$ feature point scale

P probability

$P\left(O_{o v^{j}}\right) \quad$ probability to find the object in view $\mathrm{j}$

$P\left(O_{v^{j}}\right) \quad$ probability to find feature point in view $\mathrm{j}$

$P\left(O_{o}\right) \quad$ probability to find the object

$P\left(\mathbf{T}_{o b j} \mid \mathbf{v}\right) \quad$ probability of the object pose given the input vector $\mathbf{v}$

$P_{i}\left(\mathbf{T}_{o b j} \mid \mathbf{v}\right) \quad$ probability of the object pose given the input vector $\mathbf{v}$, returned by the $i$ th decision tree

$P\left(\mathbf{T}_{o b j} \mid \mathbf{v}, \mathbf{p}_{m}^{I}\right) \quad$ probability of the object pose given the input vector $\mathbf{v}$ and the position of feature points projected in the image space

$P_{i}\left(\mathbf{T}_{o b j} \mid \mathbf{v}, \mathbf{p}_{m}^{I}\right) \quad$ probability of the object pose given the input vector $\mathbf{v}$ and the position of feature points projected in the image space, returned by the $i$ th decision tree

$P_{i}\left(\mathbf{p}_{m}^{I} \mid \mathbf{T}_{o b j}\right) \quad$ probability to find the position of feature points projected in the image space, given the object transformation $\mathbf{T}_{o b j}$

$\mathbf{T}_{o b j}^{*} \quad$ object global pose transformation matrix with maximum probability in the observation 


\section{Contents}

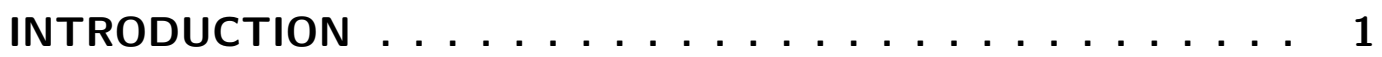

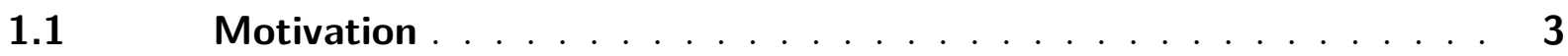

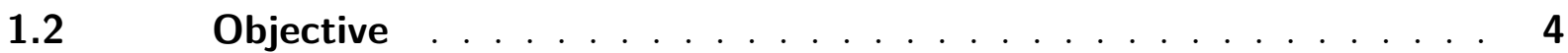

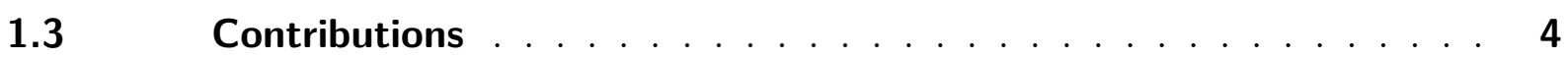

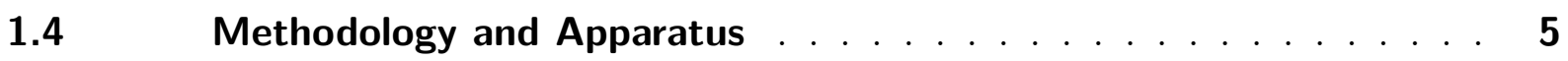

1.5 Text Organization . . . . . . . . . . . . . . . 7

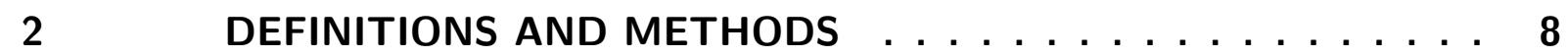

2.1 Object Based Augmented Reality and Object Classes . . . . . . . . 8

2.1.1 Rigid and Planar Objects . . . . . . . . . . . . . . . . . . 9

2.1 .2 Deformable Objects . . . . . . . . . . . . . . . . . . . . . 9

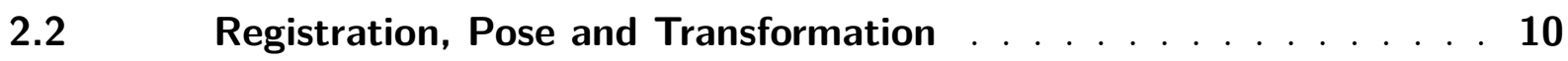

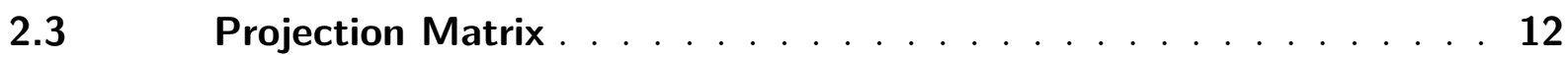

$2.4 \quad$ Local and Global Information . . . . . . . . . . . . . . 13

$2.5 \quad$ RGB frames, RGB-D frames and Depth Map . . . . . . . . . . 13

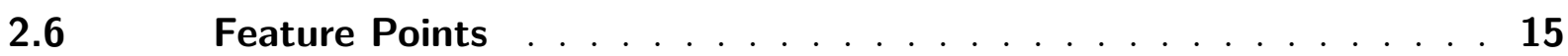

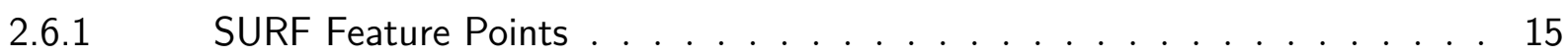

2.6.2 ASIFT: Affine transformation robust SIFT features . . . . . . . . . 16

$2.7 \quad$ Machine Learning and Decision Forest . . . . . . . . . . 17

2.7.1 Decision Forest . . . . . . . . . . . . . . . . . . . . . . . . 18

$2.8 \quad$ Chapter Conclusions . . . . . . . . . . . . . . . . . . . 19

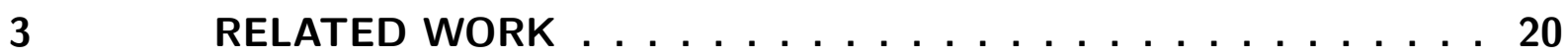

3.1 Rigid Object Pose Estimation based on RGB-D information . . . . . 20

3.2 Rigid Object Pose Estimation based on RGB information . . . . . . 24

3.2.1 Solve Perspective to $n$ Points Problem . . . . . . . . . . . . . . 24

3.3 Pose Estimation Based on Local Information and Machine Learning 31

3.4 Deformable Object Pose Estimation . . . . . . . . . . . . . . . 38

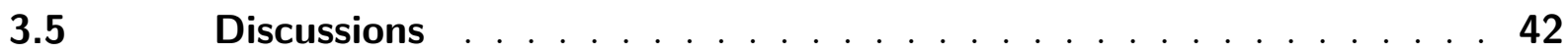

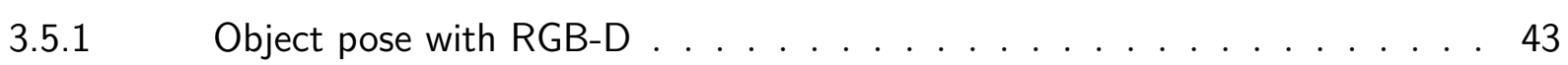

3.5.2 Object poses with RGB . . . . . . . . . . . . . . . . 44

3.6 Chapter Conclusion $\ldots \ldots \ldots \ldots \ldots$

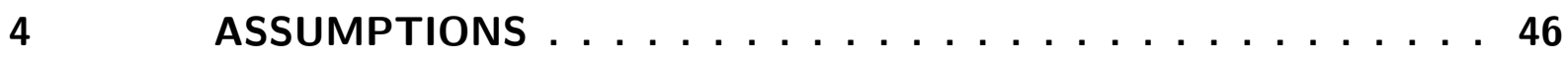

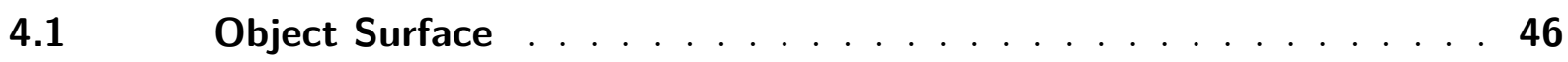

$4.2 \quad$ Capture Environment $\ldots \ldots \ldots \ldots \ldots$ 
$4.3 \quad$ Real World Scenarios _. . . . . . . . . . . . . . . . . . . 48

$4.4 \quad$ Chapter Conclusions . . . . . . . . . . . . . . . . 49

5 LOCAL POSE BASED APPROACH OVERVIEW . . . . . . . 51

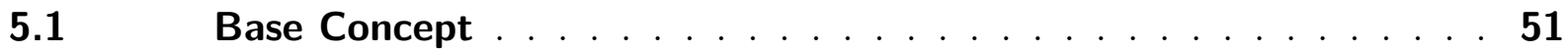

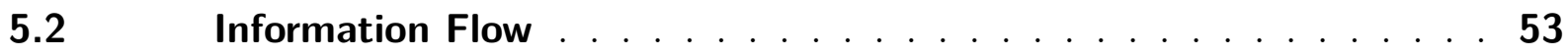

$5.3 \quad$ Chapter Conclusions . . . . . . . . . . . . . . 54

6 ROBUST FEATURE POINTS EXTRACTION . . . . . . . . 55

$6.1 \quad$ Locally Planar Assumption $A_{o 2}$ and feature Extraction . . . . . . 56

$6.2 \quad$ Background Robust Features Extraction . . . . . . . . . . . 56

6.3 Affine Transformation Robust Features Extraction . . . . . . . . 57

6.4 Entropy Maximization Feature Point Selection Method . . . . . . . 60

6.5 Chapter Conclusion . . . . . . . . . . . . . . 61

7 LOCAL POSE ESTIMATION BASED ON RGB-D INFORMATION 63

$7.1 \quad$ Pre-process of Object Feature Points . . . . . . . . . . . 63

$7.2 \quad$ Observed Feature Point Local Pose Estimation . . . . . . . . . . . 67

$7.3 \quad$ Global Pose Estimation based on Local Poses . . . . . . . . . . . . . 68

7.3.1 Planar and Rigid Objects . . . . . . . . . . . . . . . . . . . . 68

7.3.2 Deformable Objects . . . . . . . . . . . . . . . 72

$7.4 \quad$ Chapter Conclusions . . . . . . . . . . . . 72

8 REGRESSAR: DECISION FOREST LOCAL POSE REGRESSION BASED ON RGB INFORMATION . . . . . . . . . . . . . . . . 73

8.1 Regression Forest Pose Training . . . . . . . . . . . . . 74

8.1.1 Training Data Generation . . . . . . . . . . . . . . . . . 75

8.1.2 Training Feature Values Extraction . . . . . . . . . . . . . . . . . 75

8.1.3 Regression Forest Training . . . . . . . . . . . . . 76

$8.2 \quad$ Local Rotation Matrix Estimation . . . . . . . . . . . . . . 77

$8.3 \quad$ Full Transformations Matrix Estimation . . . . . . . . . . . . . 80

8.4 Global Pose of Rigid Objects: Random Forest Random Sample Consensus . . . . . . . . . . . . . . . . . . . 81

8.5 Chapter Conclusion . . . . . . . . . . . . . 83

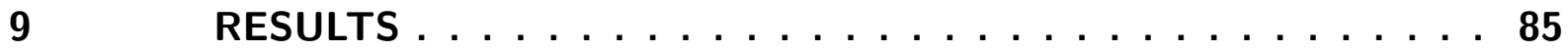

9.1 Robust Feature Point Extraction . . . . . . . . . . . . . . 85

9.2 Pose Estimation by Local Pose Based on RGB-D Information . . . . 90

9.2.1 Rigid Object Pose Estimation . . . . . . . . . . . . . . . . . . 96

9.2.1.1 Synthesized Data . . . . . . . . . . . . . . . . 97

9.2.2 Single Feature Pose Estimation . . . . . . . . . . . . . . . . . 100 
9.2.3 Deformable Object Rough Pose Estimation . . . . . . . . . . . . . . . . . 102

9.3 RegressAR: Decision Forest Local Pose Regression based on RGB Information . . . . . . . . . . . . . . . . . . . . . 104

9.3.1 Regression Forest . . . . . . . . . . . . . . . . . . . . 104

9.3.2 Rigid Object Pose Estimation based on RF-RANSAC . . . . . . . . . . 106

9.3.2.1 Synthesized Data . . . . . . . . . . . . . . . . 106

9.3.3 Deformable Object Local Rotation . . . . . . . . . . . . . . . . . . . 110

$9.4 \quad$ Result Discussions and Limitation . . . . . . . . . . . . . . . 111

9.5 Chapter Conclusion . . . . . . . . . . . . . . . . 116

10 FURTHER DISCUSSION . . . . . . . . . . . . 117

10.1 Human Perception, Feature Point Local Information and Local Pose Based Approach . . . . . . . . . . . . . . . . . . . . . . 117

$10.2 \quad$ Locally Planar Assumption $A_{o 2}$ Aside . . . . . . . . . . . . . 118

$10.3 \quad$ Light Field and RegressAR . . . . . . . . . . . . . . . 119

10.4 RF-RANSAC: Other Usage and Its Performance . . . . . . . . . 120

10.5 Local Pose And Other Methods . . . . . . . . . . . . . . . . . 121

10.5.1 Classification . . . . . . . . . . . . . . . 122

10.5.2 Tracking . . . . . . . . . . . . . . . . . . 123

10.5.3 Deformable Objects Pose . . . . . . . . . . . . . . . . . . 124

10.6 More Objects and Information for AR . . . . . . . . . . . 125

10.7 Markers for Interaction . . . . . . . . . . . . 128

10.8 Chapter Conclusion . . . . . . . . . . . . . . . . 130

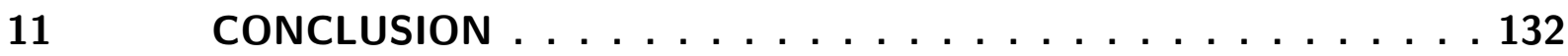

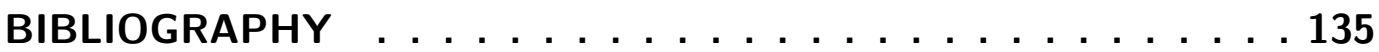




\section{Introduction}

In the past years, several applications and ideas for augmented reality (AR) have been developed (BENKO; WILSON; ZANNIER, 2014; KREVELEN; POELMAN, 2010). However, linking physical real objects with virtual information is still one central aspect on AR (KREVELEN; POELMAN, 2010). In those objects based AR, objects are mainly used as markers to obtain the visuospatial information.

This usage of real objects as link to virtual contents is not only limited to visual augmentation (COMPORT et al., 2006), but also with tactile sensations (BAU et al., 2012), as input methods (HEUN; HOBIN; MAES, 2013), or even output (LEITHINGER et al., 2014). And, a key issue for this link is the spatial registration of these objects, or in other words, the estimation of the objects poses.

Figure 1 - Results of our work applied in augmented reality applications scenarios. First row: examples of pose estimation applied in different objects; second row: concept images for applications in AR. First column: usage of real object as light source; second and third: usage of object deformation as interaction method for AR games; forth column: information visualization, extending real object visual information.
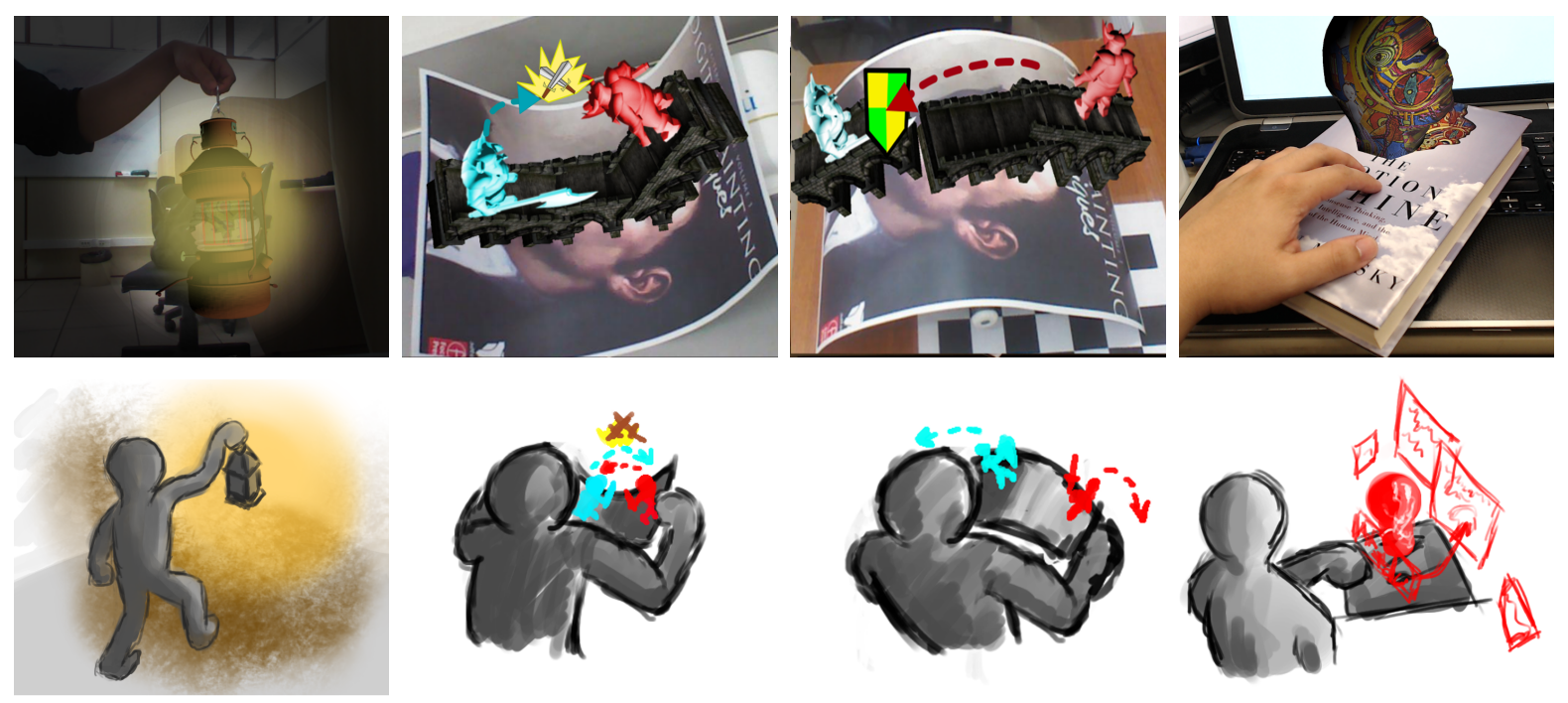

Source: Author.

On the other hand, different objects can have different behaviors when used by users. Not only restricted to rigid objects (CHOI; CHRISTENSEN, 2012) or planes (BILLINGHURST; KATO; POUPYREV, 2001) and its motion; but moreover, foldable (LEE; HUDSON; TSE, 2008), deformable (STEIMLE; JORDT; MAES, 2013), or state changing (MAGNENAT et al., 2015) objects. Such objects can offer different kinds of interaction possibilities, such as illustrated in Figure 1. However, for each class of objects, 
different methods had been presented in the literature in order to obtain such objects poses.

Moreover, conventional methods for pose estimation, rigid or deformable, are often based on correlation of known points of the object with the observed points (LEPETIT; MORENO-NOGUER; FUA, 2009; PILET; LEPETIT; FUA, 2008; HENRY et al., 2012). Those methods simplify the observed and known information to simple points in space. However, the object itself can contain much more information. Figure 2 illustrates this simplification. Other than the point position, characteristics such as appearance around interest points (figure 2d) or the surface information (figure 2c) also can be used for pose estimation.

Figure 2 - Information loss in different cases. Conventional methods simplify the object full information $2 \mathrm{a}$ to simple points $2 \mathrm{~b}$. However, more information can be gathered, such as local surface normals $2 \mathrm{c}$ or local appearance $2 \mathrm{~d}$.

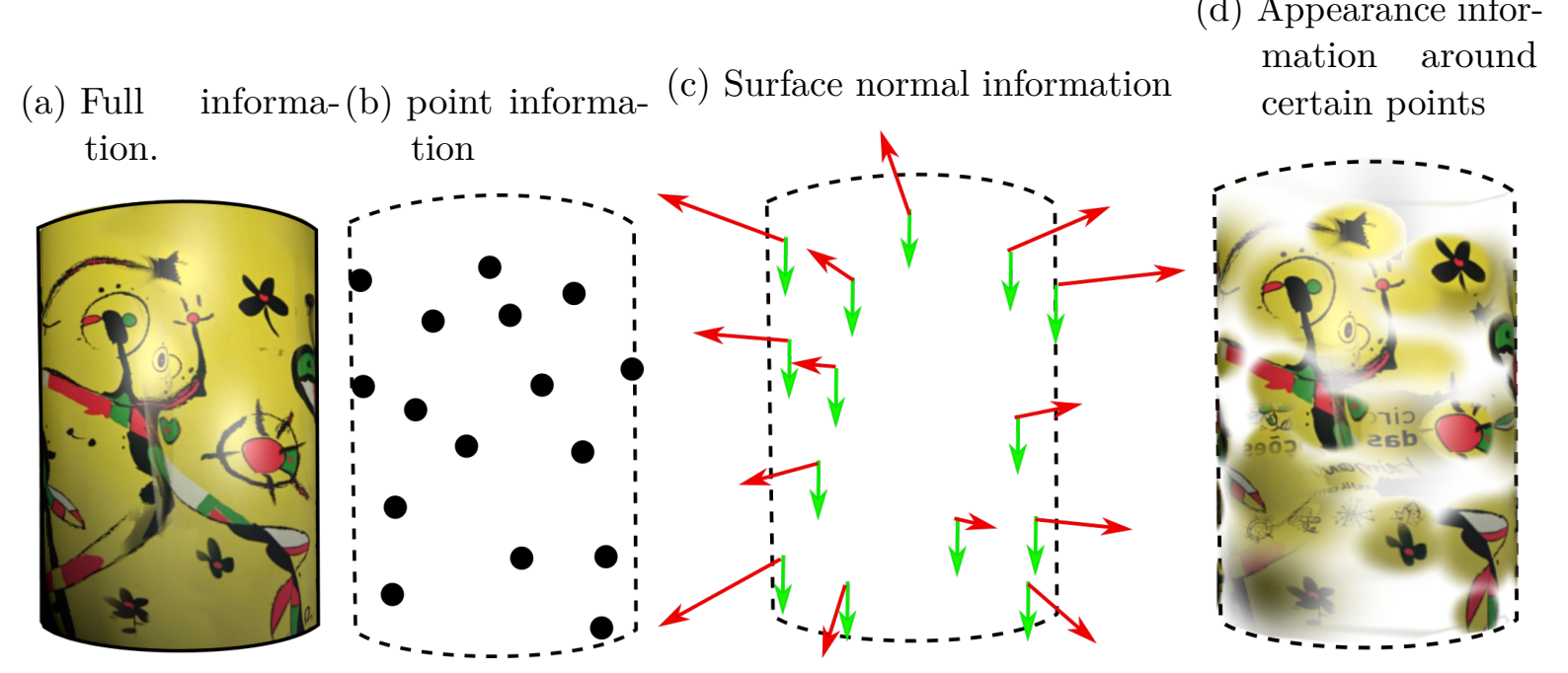

Source: Author.

Additionally, we observed through several works (chapter 3) that several object types share one common characteristic; to be locally rigid. This means that local properties of the object do not change, even when the object shape is changing. By knowing how locally the object pose is changing, it is possible to estimate the entire pose of these objects.

These characteristics give foundation to this work main idea; to obtain poses of different classes of objects, based on local poses of the object estimated by gathering local information. Full local poses or rotations are not explored in literature, although they can give rich information for several cases. Thus, our main hypothesis in this work is:

Gathering more local information from each feature point can give us rich information to estimate the pose several types of objects. 
In order to explore this hypothesis, we have applied this idea in two different capturing setups, using RGB-D and RGB cameras. In each setup, we have reached to create novel approaches of estimation of local and global object poses for augmented reality (chapters 7 and 8). We also extended feature points extraction methods in order to support our local pose estimation.

\subsection{Motivation}

Usage of real objects as markers to obtain the visuospatial registration is a widely studied problem, by several authors (XIAO; TOME; ISHII, 2014; ISHII; MAZALEK; LEE, 2001). Azuma (1997), one of the first works to define augmented reality, already explored the idea of registration of real object for augmentation using virtual content, even from the early time of AR.

Usage of fiducial markers for pose estimation was widely explored in AR (BILLINGHURST; KATO; POUPYREV, 2001). Those markers use known patterns to obtain the registration of the real world. However, they often use high-contrast pattens that appear unnatural at the scene (LIMA et al., 2010; CHIA; CHEOK; PRINCE, 2002).

Alternatively, scale and rotation invariant image feature points extraction and matching works (LOWE, 1999; BAY et al., 2008) enabled the use of natural objects (LIMA et al., 2010) or scenes to be used for registration (CHIA; CHEOK; PRINCE, 2002). Works such as (XIAO; TOME; ISHII, 2014; HINSKE; LANGHEINRICH; LAMPE, 2008; GRASSET; DüNSER; BILLINGHURST, 2008) explore those natural feature points for object pose estimation and its usage in augmented reality.

Now, if we point the spotlight to the object interaction itself, we can observe that the manipulation of those objects can bring rich interaction and visualization methods (RENDL et al., 2014; ISHII; MAZALEK; LEE, 2001). Even the straight forward usage of the object pose in the scene can be explored for data visualization and interaction (ISHII; MAZALEK; LEE, 2001).

Moreover, interaction with different types of real objects can also bring novel approaches for computer interaction and AR (RENDL et al., 2014; LEE; HUDSON; TSE, 2008; STEIMLE; JORDT; MAES, 2013). Several works explore the deformation of the object for visualization and content interaction (RENDL et al., 2014; LEE; HUDSON; TSE, 2008); folding of the object are also explored as interaction method (STEIMLE; JORDT; MAES, 2013). Changing the object appearance or state is also another way of interaction, such as painting a real image to change the virtual character appearance (MAGNENAT et al., 2015), or obtaining different simulations of the drawn objects (HAGBI et al., 2008).

Thus, we can observe that usage of real object for interaction as markers, linking 
the real world and the virtual information is one key factor in AR. And, in order to obtain this link, the estimation of the object pose plays central role (AZUMA, 1997). Despite that, literature lacks on a common method or approach to estimate the pose of different objects with different behaviors.

\subsection{Objective}

Our objective in this work is to explore local information of feature points to estimate local poses of a known object, in both RGB and RGB-D capture conditions. Then, based on those local information, obtain full pose of different classes of objects. In order to be able to use those objects as markers for augmented reality systems.

\subsection{Contributions}

Our main contribution in this work is the idea of global pose estimation based on local pose information of small patches of the object surface itself. Furthermore, our contribution extends to:

- robust feature point extraction method;

- local and global pose estimation approach based on RGB-D cameras and local surface normal and orientation;

- local and global pose estimation approach based on RGB cameras and decision forest regression and RF-RANSAC.

First, in order to gather information of feature points across several poses in realtime conditions, we extend an affine invariant feature point extraction method (MOREL; YU, 2009). Moreover, we apply an entropy based filtering of affine transformation robust feature points to obtain a balance between view coverage and observation probability.

Then, based on RGB-D cameras, we gather information about surfaces normal and orientation of feature points in order to fully estimate the local pose of feature points. Unlike other works in literature, we explicitly obtain the local poses, applying them for estimation of rigid and deformable objects poses.

Finally, we propose an approach for pose estimation based on RGB cameras and machine learning. Conventional methods do not rely on local information, thus, can not extract local poses; and also rely on complex non-linear equation solvers. Other works apply a machine learning approach to replace such solvers, but they do not obtain full 6 DoF pose. We are the very first to obtain full object pose based on machine learning approaches, and also, obtain local rotation of each feature point. Further discussions about 
the differences of our approaches for pose estimation with conventional methods will be presented at chapter 3 .

Also, as secondary contributions, we have achieved:

- single feature pose estimation of planar objects with RGB-D information;

- simple deformable object rough pose estimation in RGB-D information;

- full 6 DoF object pose based on machine learning and RGB information;

- local rotation estimation of deformable objects based on RGB information;

applying our methods in different classes of objects.

\subsection{Methodology and Apparatus}

Initially, we have conducted a literature review about methods for object pose estimation which can be used for AR. From this research, it was possible to observe that such works can be separated by two major factors; the capturing setups and the type of the object.

Capturing setups can vary for different works, which can apply doppler effec speed sensors (MIYASHITA et al., 2015), computational photography (VEERARAGHAVAN et al., 2007; MARWAH et al., 2013) or controlled illumination (HOLROYD et al., 2008; HERTZMANN; SEITZ, 2005). However, the most common setups are the ones based on conventional RGB cameras; and the ones based on depth and color cameras, RGB-D cameras.

Also, literature can vastly differ according to the object type. Several works focus on pose estimation of rigid objects (CHOI; CHRISTENSEN, 2013; HENRY et al., 2012; LEE; NGUYEN, 2014; LOWE, 1999; LEPETIT; MORENO-NOGUER; FUA, 2009). However, objects that can vary its shape across time are also focus on works such as (RENDL et al., 2014; PILET; LEPETIT; FUA, 2008; PAUWELS; RUBIO; ROS, 2014; STEIMLE; JORDT; MAES, 2013). Thus in this work, we have separated the object types in two categories, the rigid and deformable objects. This separation is done to be able to analyze if our approaches can be applied for poses estimation of different types of objects.

Both RGB and RGB-D setups had become popular in literature and even in consumer market. Thus, we also applied our local pose idea to both of the capturing setups. Generating two different approaches for object pose estimation from the same local pose idea. 
Once, both approaches are based on feature points, we also created a method for affine transformation robust feature points. This can give us feature points that can be observed across different scales, rotations and affine transformations of the object.

For the evaluation of our results, we had taken an approach of synthesizing the ground truth data using graphical render languages. By rendering images and depth maps of the object in different poses, we can obtain the ground truth data of those poses, and compare our results with the true pose values. This approach is similar to (LEPETIT; MORENO-NOGUER; FUA, 2009; LI; XU; XIE, 2012; ZHENG et al., 2013). Those synthesized data are also used in training and pre-processing parts of our methods.

Apparatus used in this work are mainly related to capturing image data from the object. For color (RGB) image capturing, we used three different cameras, illustrated in figure 3. First, the RGB camera used in Kinect sensors ${ }^{1}$, which can also capture the depth information of the scene (RGB-D).

The second camera is the Logitech C920 webcam ${ }^{2}$, a common, but with mid-quality optics for a small lens system. Webcams are widely used capturing system in AR, and also are similar to some optical system used in handheld devices, such as smartphones. Thus, we chose this camera to represent conventional AR capturing systems.

At last, we also captured the object RGB information using a Digital Single-Lens Reflex (DSLR) Nikon D3200 ${ }^{3}$ camera, which has superior optics system compared to the other two cameras. It also has a mid-size APS-C sensor, capable of high-resolution images. Despite that, we do not use its full resolution, and resize it to similar sizes to Kinect and Logitech webcams, to be able to obtain similar conditions.

Figure 3 - Apparatus used in this work.

(a) kinect camera

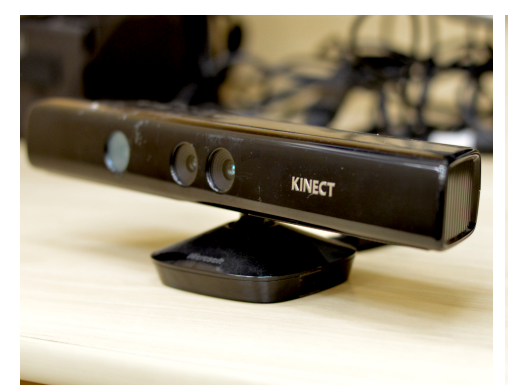

(b) logitech C920 webcam

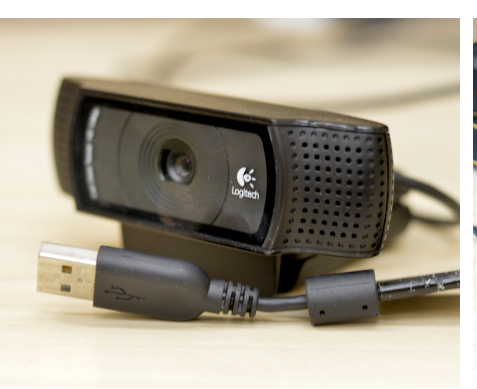

Source: Author. (c) Nikon D3200 camera

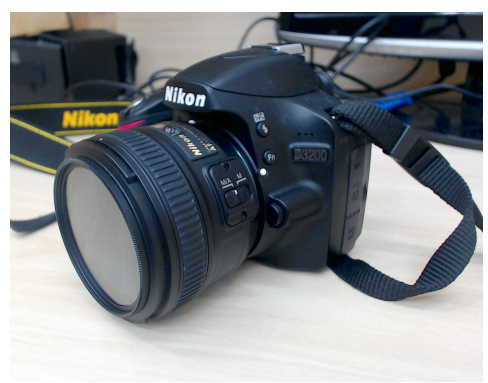

For the generation of synthesized images for test and training, we used the Processing

$<$ https://dev.windows.com/en-us/kinect>; last accessed at february of 2016

$2<$ http://www.logitech.com/en-us/product/hd-pro-webcam-c920>; last accessed at february of 2016

$3<$ http://www.nikonusa.com/en/nikon-products/product/dslr-cameras/d3200.html>; last accessed at february of 2016 
${ }^{4}$ programming language. Which is based on OpenGL ${ }^{5}$ graphical language. This rendering library generates similar results to real objects, if they can be modelled as Lambertian surfaces (ANGEL, 2006). Finally, most of the algorithms and methods of this work are implemented using MATLAB ${ }^{6}$ environment. Although, it lacks on computational performance, MATLAB provides a variety of methods and analysis tools; such as the decision forest training tool used in this work.

\subsection{Text Organization}

In the following chapters, we present our pose estimation methods. First, in chapter 2, we present definitions, concepts and some methods used in this work, in order to stablish a common knowledge base, used across this work. Then, chapter 3 presents state-of-art works in literature, and their different approaches for object pose estimations. Also, we present discussion about differences that our approaches have with those works.

Chapter 4 describes assumptions adopted in this work about the object model and capturing system. Also, about how these assumptions can be reached in real world scenarios.

We present the overview of our work in chapter 5; presenting the general idea behind this work, as well as, the approaches to obtain the object pose in different capture conditions, and those information flow.

Chapter 6 describes the method used to extract feature points of the object for the pose estimation process. Then, chapters 7 and 8 describe the approaches and methods created to estimate the local pose of feature points and the global pose of objects.

In chapter 7 , we describe the pose estimation method based on a setup using RGB-D cameras. On the other hand, in chapter 8, we describe the local pose estimation method using only conventional RGB cameras. All the results obtained in this work and their discussions are presented in chapter 9.

Chapter 10 presents further discussions about our approaches, how they are related with other approaches and methods in literature, as well as, possible applications of our approaches in augmented reality and entertainment. Finally, in chapter 11 we describe our conclusions in this work and possible future works.

\footnotetext{
$4<$ https://processing.org/>; last accessed at february of 2016

$5<$ https://www.opengl.org/>; last accessed at february of 2016

6 <http://www.mathworks.com/products/matlab/>; last accessed at february of 2016
} 


\section{Definitions and Methods}

In this chapter we present the essential definitions and methods, for the sake of understanding of this work. We present a detailed view of them, as well as, their characteristics, once they will be used across this text.

\subsection{Object Based Augmented Reality and Object Classes}

Augmented reality (AR) can be defined as the interactive combination of real and virtual information in real-time and registered to each other, as defined by Azuma (1997). From this definition, it is possible to observe that the 3D registration of objects is one of the key points in AR.

This registration can be achieved by knowing how the viewer (or camera) is placed in the world (HENRY et al., 2012), or by pose estimation and tracking of known objects in the scene (LIMA et al., 2012), used as marker, and combining the virtual information in reference to these markers. The first is often based on technologies as environment tracking and mapping as SLAM (simultaneous localization and mapping) (DAVISON, 2003) or PTAM (parallel tracking and mapping) (KLEIN; MURRAY, 2007), witch try to localize the viewer (camera) position and map the environment, independently of what objects constitutes this mapped environment.

On the other hand, the second is related with object tracking (YILMAZ; JAVED; SHAH, 2006), pose estimation (LEPETIT; MORENO-NOGUER; FUA, 2009) and classification methods (FENZI et al., 2013; LIN et al., 2015). These augmented reality based on object has the characteristics of being directly related with the object to be tracked as in (STEIMLE; JORDT; MAES, 2013; ISHII; ULLMER, 1997), once it also needs to classify the object. Also, once real objects are used to augment the world, they often are related to tangible interfaces (ISHII; ULLMER, 1997).

We define object based augmented reality as a ramification of AR which uses known objects as markers to obtain the 3D registration, as presented above. We also can observe that the object to be estimated or tracked is a central issue of object based AR. These objects can have different semantic and physical characteristics, providing different interactions, storytelling and contexts (ISHII; ULLMER, 1997; STEIMLE; JORDT; MAES, 2013; LEE; HUDSON; TSE, 2008).

In this work, we also separate these objects themselves in two major classes, according to its physical characteristic related to pose estimation: 
- rigid objects;

- deformable objects.

The first class are the rigid objects, which keeps its shape across the time (AKENINEMOLLER; MOLLER; HAINES, 2008). Rigid objects are often found in real world and are commonly used in AR systems (ISHII; ULLMER, 1997; LIMA et al., 2010; FISCHLER; BOLLES, 1981). The second type of objects are the deformable ones. Deformable objects are objects that can change its shape across observations (WATT, 1993; AKENINE-MOLLER; MOLLER; HAINES, 2008).

\subsubsection{Rigid and Planar Objects}

Rigid objects can be defined as objects which the spatial relation of its points does not change across time (AKENINE-MOLLER; MOLLER; HAINES, 2008). Meaning that, the spatial relation of the surface feature points also do not change from the pre-processed model and the estimation of the pose in the new frame. Also, this means that all the points has the same transformation across the capturing.

These characteristics makes this class of objects pose easier to be estimated, since only one transformation matrix needs to be found. Therefore, the object pose can be represented as only one transformation matrix $\mathbf{T}_{o b j}$ that represents all the transformation of all points.

Each point $\mathbf{p}^{o}$ in the object space can be converted to the camera space positions, $\mathbf{p}^{w}$, by multiplying the transformation matrix $\mathbf{T}_{o b j}$, as

$$
\mathbf{p}^{w}=\mathbf{T}_{o b j} \mathbf{p}^{o}
$$

Planar objects are a special case of rigid object, where all of its feature points are co-planar. This characteristic makes planar objects pose easier to be estimated, such as presented in works based on homography estimation (CHUM; PAJDLA; STURM, 2005), widely used on AR (PRINCE; XU; CHEOK, 2002; MALIK; MCDONALD; ROTH, 2002), or on fiducial markers (BILLINGHURST; KATO; POUPYREV, 2001) (which has a special pattern to be found and estimated). Despite that, we consider both objects, 3D or planar, as the same class of rigid objects in this work. Therefore, its pose can be estimated with same method.

\subsubsection{Deformable Objects}

Another class of object used in augmented reality are the deformable objects (LI; SUMNER; PAULY, 2008; SUMNER; SCHMID; PAULY, 2007). Deformable objects are objects that can change its shape freely. Thus, making these objects poses harder to be 
estimated, since can not be represented by a single transformation matrix. Several methods try to estiamte the pose using different assumptions about the object (PRITCHARD; HEIDRICH, 2003; PILET; LEPETIT; FUA, 2008; CHANG; ZWICKER, 2011; RENDL et al., 2014; JORDT; KOCH, 2013). On the other hand, these objects are also used as medium to novel interactions methods, since they can allow different transformation of the object as bending or folding (RENDL et al., 2014; STEIMLE; JORDT; MAES, 2013; LEE; HUDSON; TSE, 2008).

Defining deformation can be hard, once different types of deformations can happen. Thus, several constraints need to be considered for pose estimation. Figure 4 illustrates some of possible types of deformations. Each type of deformation needs different constraints, and needs different methods for pose estimation.

Figure 4 - Examples of possible deformations of the same planar object.

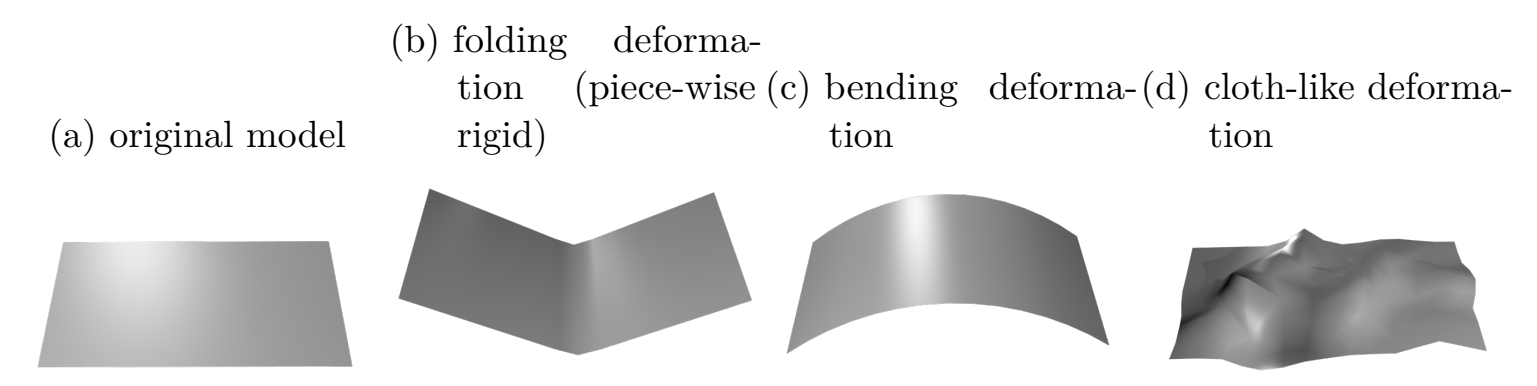

Source: Author.

One way to define the object deformation is by knowing the position of the points spread across the object surface (PILET; LEPETIT; FUA, 2008). By knowing those points position, the transformation applied to each polyogonal face formed by those points can be estimated. Thus, giving the transformation of the surface which gives the global deformation. Figures 5a and 5b illustrates this condition. However, this also means that knowledge of the local transformations of the surface can also gives us the deformed object pose, as illustrated in figures $5 \mathrm{c}$ and $5 \mathrm{~d}$.

\subsection{Registration, Pose and Transformation}

Due to research areas covered in this work, some definitions can have similarities with slight differences. One case, are the definitions of registration, pose and transformation. "Registration" is a definition used in AR, related specially to spatial coherence of the virtual object with the real world (AZUMA, 1997). This registration is also strongly connected with the relation of the user view with real objects.

On the other hand, "pose" is a definition specially used in computer vision researches, which is defined as the spatial state which some object is in some determined time 
Figure 5 - Possible deformation estimation based on points. First row: based on point positions; second row: based on local transformations.

(a) original model points information

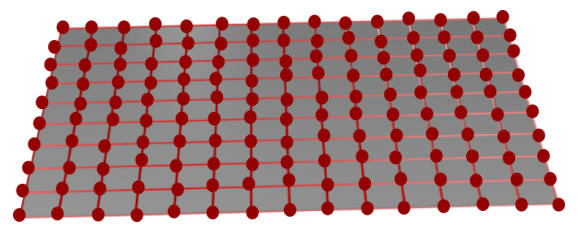

(c) original model local transformations

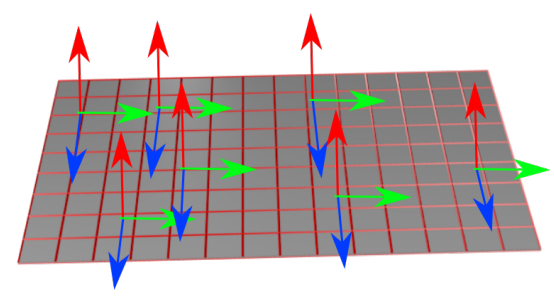

(b) deformed points information

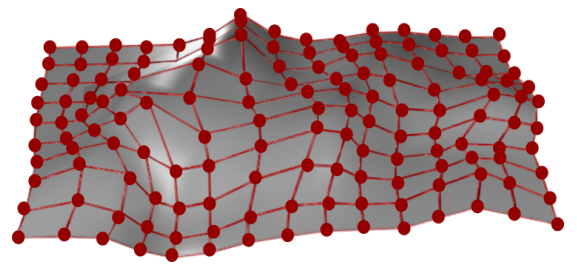

(d) deformed model local transformations

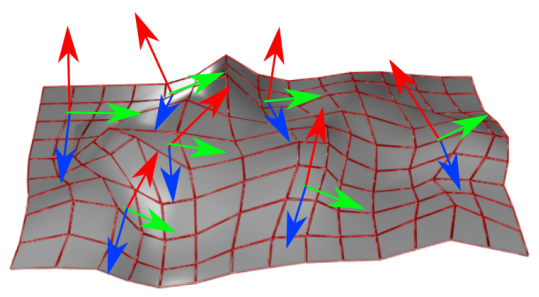

Source: Author.

(FAUGERAS, 1993). In case of rigid objects in 3D scenes, this pose can be expressed as the 3D position and rotation that defines where the object is (AKENINE-MOLLER; MOLLER; HAINES, 2008). In case of deformable objects, this pose is determined by how the shape is transformed, such as in human bodies pose estimation (SHOTTON et al., 2011). As well as, camera poses can be defined as the positioning of the camera related to some scene, as shown in some works as (HENRY et al., 2012; PRINCE; XU; CHEOK, 2002).

Finally, "transformation", or "transformation function", can be defined as a function that converts some known spatial state to another, and widely used in computer graphics (AKENINE-MOLLER; MOLLER; HAINES, 2008) and computer vision works (FAUGERAS, 1993). "Transformation matrices" are matrices that contains transformation information of translation and rotation, and when multiplied with some vector or point, returns the new position of that vector or point (AKENINE-MOLLER; MOLLER; HAINES, 2008).

The AR "registration" can be achieved by knowing the "pose" of the scene or objects. Yet, is important to observe that, "pose" is not the same as "registration" in AR. This is due to he fact that, if the virtual object is related with some specific object, knowing the pose of the camera in a scene, still does not gives us the registration, once the object can be freely moving across the scene. And the inverse is also true; when you need to register virtual information in the real scene, knowing the pose of an object does not gives the registration of this scene, once this object can be moving inside the scene. However, in this work we focus on object based augmented reality, where the virtual objects are 
always related with real objects. Therefore, knowing the real object pose leads us to the AR registration.

The object "pose" can be represented as a "transformation" function, that can be a "transformation matrix" or a collection of them. Such as in works of camera pose estimation or rigid object pose estimation, where the "transformation matrix" of the scene or object gives us the respective "poses" (LEPETIT; MORENO-NOGUER; FUA, 2009; LOWE, 1991).

Therefore, we can state that: object based AR "registration" can be achieved by knowing the "pose" of some object, which in turn, can be represented by a "transformation" function. Thus, in this work, we define "registration" as the coherence between virtual and real objects, "pose" as the spatial state of the real objects, and "transformation", the function that maps the known state of objects into some other spatial state, normally to the observed object state.

\subsection{Projection Matrix}

As defined by (AKENINE-MOLLER; MOLLER; HAINES, 2008; FAUGERAS, 1993), projection matrix is a $n \times n$ matrix that gives the projection of some point in a $\mathbb{R}^{n}$ space to a subspace $\mathbf{W}$. In computer graphics or vision, this projection matrix is often used in order to obtain the perspective projection $\mathbb{R}^{2}$ of some scene in $\mathbb{R}^{3}$, in order to obtain an image that represents the view of this scene, as in rendering pipelines (AKENINE-MOLLER; MOLLER; HAINES, 2008), also called as perspective projection matrix.

This matrix, also named as intrinsic matrix or camera matrix (FAUGERAS, 1993), contains parameters that converts the world scene into the image space, conventionally named as intrinsic parameters. This is done by multiplying the homogeneous coordinates of the scene to the matrix. The homogeneous coordinates are the Euclidean space coordinates divided by the last $\mathrm{Z}$ value, giving us:

$$
\begin{array}{r}
p^{I}=K_{p} \mathbf{p}^{H} ; \\
\mathbf{p}^{3 D}=\left[X^{3 D}, Y^{3 D}, Z^{3 D}\right]^{T} \\
\mathbf{p}^{H}=\left[X^{3 D} / Z^{3 D}, Y^{3 D} / Z^{3 D}, 1\right]^{T} .
\end{array}
$$

Here, $K_{p}$ is the perspective projection matrix, $\mathbf{p}^{3 D}$, the Euclidean position of some point and $\mathbf{p}^{H}$, the homogeneous coordinates of $\mathbf{p}^{3 D}$, and $p^{i}$, the position at the image.

One type of the intrinsic parameters is the focal length, $f_{x}$ in $\mathrm{x}$ dimension and $f_{y}$ in $\mathrm{y}$. These parameters are related with the projected position of some point at the image plane and focal position; also with the camera field of view. The field of view can be defined as the view angle of the projected image, defining how much are that the camera 
can collect light. Both can be converted to each other with the equation

$$
\phi=2 \arctan \left(\frac{w}{2 f}\right)
$$

where $\phi$ is the field of view angle, $w$ is the size of the projection plane, the width in pixels in case of the $\mathrm{x}$ filed of view of an digital image, and $f$ is the focal position (AKENINE-MOLLER; MOLLER; HAINES, 2008).

In this work, we define as "projection matrix" the perspective projection matrix that contains the intrinsic parameters and gives us the image position of a 3D point at the image plane, when multiplied as in equation 2.2 .

\subsection{Local and Global Information}

One key aspect explored in this entire work is the idea of exploring local information. In contrast to global information which cover the entire object, local information are ones visible only around a certain point (LEPETIT; LAGGER; FUA, 2005; LI; SUMNER; PAULY, 2008).

More specifically, in this work, we define the local information, information that can be collected or estimated around some feature point $\mathbf{p}$, within an area roughly formed by an circle with radius related to the scale $s$ which the feature point is found. On the other hand, other information out of this area are considered to be global information, such as other feature points. Figure 6 illustrates this local/global concept.

\subsection{RGB frames, RGB-D frames and Depth Map}

RGB image information returned by image sensors, such as CCD (HAIN; KAHLER; TROPEA, 2007; CHEN; GINOSAR, 1995) or CMOS sensors (GAMAL; ELTOUKHY, 2005; FOSSUM, 1995), can contain several visual information, and are widely used in order to obtain information of the scene, as classifying objects (LIN et al., 2015). However, once they are a projection of the incoming lights in a plane, they lack in 3D spatial information.

On the other hand, depth maps are information projected into a planar representation, as an image, which contains spatial distance information between the camera and some object or surface in a determined directions. Once they contain spatial information, they can be used in a wide variety of application, as 3D reconstruction of real environments and objects (HENRY et al., 2012; SHOTTON et al., 2011).

These depth maps can be achieved by several methods, as structured lights (SALVI; PAGES; BATLLE, 2004), which projects known patterns over the surface of objects and analyze the displacement of the pattern. Depth maps also can be achieved by time-of-flight 
Figure 6 - Local and global information differences. Feature point $\mathbf{p}_{i}$, has as local information of normal $\vec{n}_{i}$, orientation $\vec{o}_{i}$ and scale $s_{i}$, represented in red color. Other point information $\mathbf{p}_{j}$, normal $\vec{n}_{j}$, orientations $\vec{o}_{j}$ and scales $s_{j}$ are considered global information to $\mathbf{p}_{i}$, represented in black color.

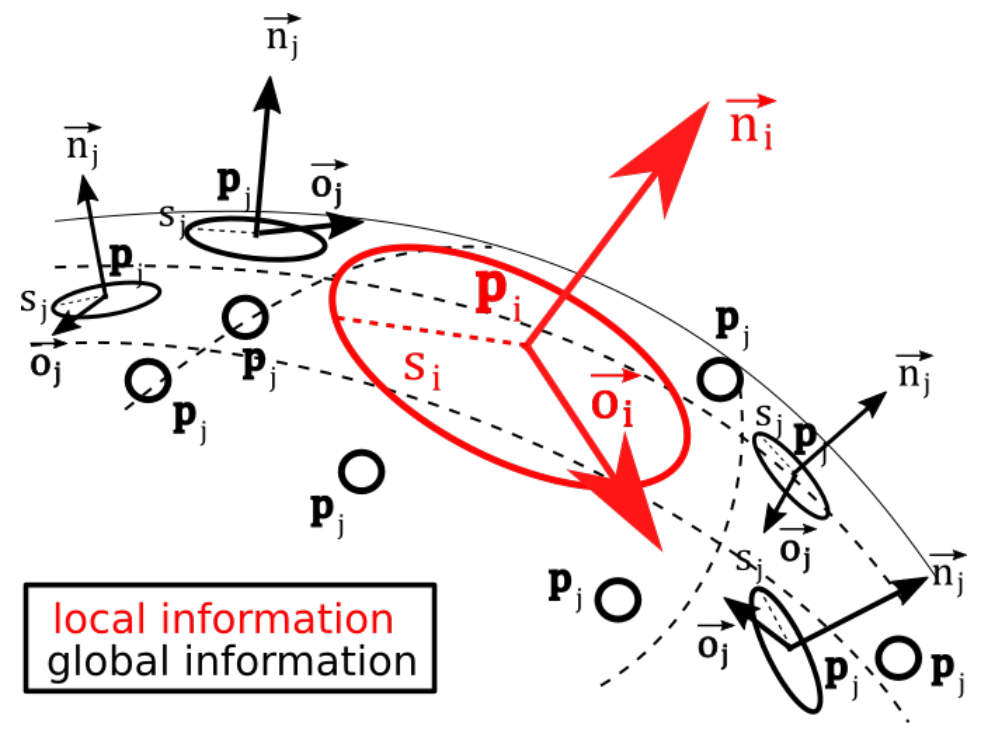

Source: Author.

(ToF) sensors, which project fast switching light pulse sequence and analyze the frequency offset by a convolution of the outgoing and incoming light. Laser patterns and scanning (MARSHALL; STUTZ, 2011), photometric stereo (HOLROYD et al., 2008), Light Field (WANNER; GOLDLUECKE, 2012; KIM et al., 2013), also are used in order to achieve the depth information, but they rarely can achieve real-time sampling of a entire image. More over, cameras based on structured light and time-of-flight can easily be found as off-the-shelf products today ${ }^{1}$.

Despite the wide usage, depth maps lack in photo reflectance information of the scene. Combination of both photo and spatial information can bring extremely rich information in order to classify (BRACHMANN et al., 2014), map and reconstruct (HENRY et al., 2012), and off course, estimate the pose of several objects (SHOTTON et al., 2013) and scenes (LIMA et al., 2012).

In this work we define as RGB frames or RGB information, image information returned by visual sensors; depth maps, as range information of the scene; and RGB-D frame, or RGB-D information, combinations of both depth and RGB information.

\footnotetext{
1 such as kinect 360 sensor <http://www.xbox.com/en-US/xbox-360/accessories/ kinect $>$ intel RealSense <https://software.intel.com/en-us/blogs/2015/05/21/ cappasity-3d-scan-with-intel-realsense-technology $>$, kinect one sensor < http://www.xbox.com/en-US/ xbox-one/accessories/kinect-for-xbox-one>, Mesa ToF SR4000 <http://www.adept.net.au/cameras/ Mesa/SR4000.shtml>.
} 


\subsection{Feature Points}

In order to estimate the object pose based on images, an important task is to detect interest points at the image. These interest points can be generated by several types of information, as edge (DALAL; TRIGGS, 2005), edgels (WERNECK; COSTA, 2013), blobs (BAY et al., 2008; LOWE, 1999), or ones that describe characteristics of certain points such as HoG features (DALAL; TRIGGS, 2005). One special type of interest point are the ones with features that can lead to scale and rotation invariant detection.

In this work, we use the term "feature points" as interest points that has scale and rotation invariant features, such as extracted by SIFT (LOWE, 1999) and SURF (BAY et al., 2008) methods. This characteristics are widely explored by several state-of-art works (HE; SIGAL; SCLAROFF, 2014; LEPETIT; MORENO-NOGUER; FUA, 2009; TORKI; ELGAMMAL, 2011; FENZI et al., 2013).

Conventionally, scale invariance is achieved by the detection of interest points in several scales. In case of SIFT features, this is done by calculating the differences of gaussians (DoG), which is a approximation of Laplacians of Gaussians (LoG), with several Gaussian variances values. And rotation invariance, by using major directions of the feature point and values relative to it (LOWE, 1991).

HoG features are also well known in the literature. However, they lack in rotation and scale invariance (STEFANOU; ARGYROS, 2012). Thus, we will refer to "feature points" whenever they are rotation and scale invariant, not including HoG features, unless explicitly written, in order to avoid confusions.

\subsubsection{SURF Feature Points}

SURF features is another feature points detection/description method that achieve rotation and scale invariance as SIFT features. However, its detection and matching speed is greater than SIFT, without precision lost in comparison to other feature detection/description methods. This characteristic makes SURF features better for real-time systems (BAY et al., 2008), and for this reason, we use SURF points as base method for feature points detector and descriptor in this work.

In SURF features, the interest points are detected by Laplacian of Gaussians (LoG). Bay et al. (2008) approximate the LoG by a set convolutions of Box Filters with several sizes $s$, that creates the scale invariance of the feature point. This approximation can be faster and enable the process in parallel of different scales, once the box filter can be easily calculated by integral images. Creating blob-like interest point detections.

After the detection, dominant orientation of the feature point is calculated by a Wavelet response of the image patch detected at scale $s$. The dominant orientation is 
calculated by the sum of all responses in $d_{x}$ and $d_{y}$, also calculated by the integral images.

Finally, the SURF descriptor is obtained by, again, the Wavelet response in the vertical $d_{y}$ and horizontal $d_{x}$ direction, using the integral image. For this purpose, SURF features take a $20 s \times 20 s$ neighbourhood of the interest point, where $s$ is the detected scale. SURF separates this region in $4 \times 4$ subregions, and creates a vector of $v=$ $\left(\sum d_{x}, \sum d_{y}, \sum\left\|d_{x}\right\|, \sum\left\|d_{y}\right\|\right)$ of each subregion in relation to the orientation, generating a descriptor vector of 64 dimensions with the rotation invariance.

As presented above, SURF features can reach higher performance than SIFT features, once the box filter convolution using integral images are used all across the detection and description extraction. This makes SURF features faster then SIFT features, a characteristic desired in real-time applications, such as in AR applications.

However, as pointed by (MOREL; YU, 2009), SURF feature points detector and descriptor alone still can not achieve full invariance to affine transformations, neighther SIFT feature descriptors. In order to extract full affine transformation robust interest points, other solutions need to be applied over these conventional feature point detection/description. In special, we focus in the ASIFT (MOREL; YU, 2009) approach for affine transformation robust feature points extraction in this work.

\subsubsection{ASIFT: Affine transformation robust SIFT features}

Several variants and extensions of SIFT features such as PCA-SIFT (KE; SUKTHANKAR, 2004), GLOH(gradient location-orientation histogram)(MIKOLAJCZYK; SCHMID, 2005), MSER(maximally stable extremal region) (MATAS et al., 2004) and LLD (level line descriptor)(MUSÉ et al., 2003) try to address the problem of affine invariant, yet, as pointed by (MOREL; YU, 2009), they are not fully affine invariant. On the other hand, ASIFT features (MOREL; YU, 2009) achieve full affine transformation of feature points by brute force detection and matching of fully simulated affine distortions between both images to match.

In order to achieve this full affine invariance, Morel e Yu (2009) initially render both images with several camera positions, simulating the affine distortions. In their work, virtual cameras are placed in positions with longitude values $\theta=\arccos (t)$, where, $t=a^{0}, \ldots, a^{n}$; and latitude values $\phi=b / t, \ldots, k b / t$; all pointing toward the image center. Figure 7a illustrates this camera model.

This cover a relatively dense sampling of affine deformations of the feature points. After both images are deformed, the algorithm compares all the extracted feature points of both image sets. Camera positions matching illustration are illustrated in figure 7b.

This simulated camera perspective changes, cover almost all of the affine space as pointed by authors (MOREL; YU, 2009), once the scale and rotation invariance of 
Figure 7 - ASIFT feature points process extraction and matching.

(b) ASIFT matching process

(a) ASIFT camera model
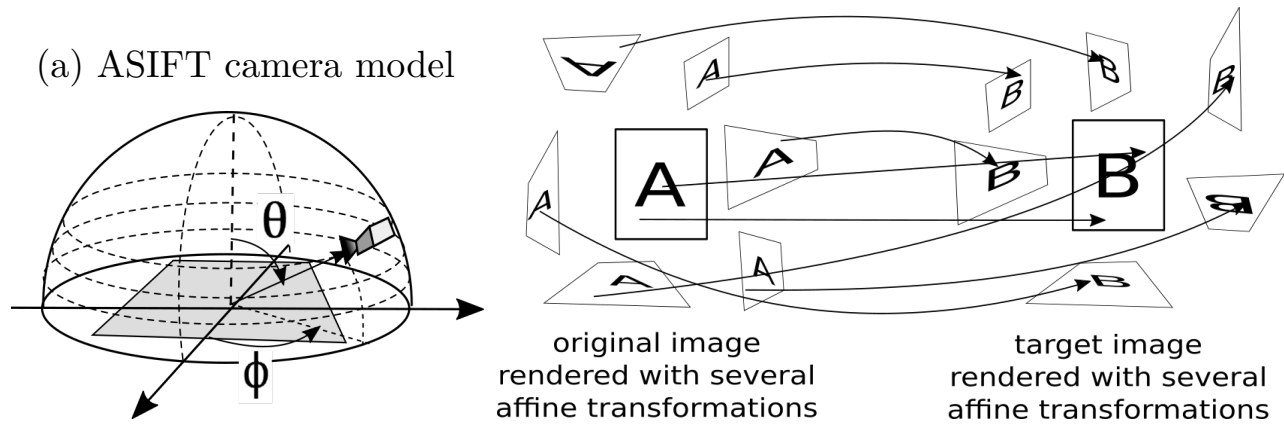

Source: Author, based on (BAY et al., 2008).

SIFT features already cover the rotation of camera axis and z position in theory. However, this approach creates a large number of feature points of the same images in order to compare, and also needs the simulation of perspectives, done by rendering the image. This characteristics makes the ASIFT hard to use in real-time applications. We present, in this work, a variant of the ASIFT method in order to be used in real-time applications, at chapter 6.

\subsection{Machine Learning and Decision Forest}

Murphy (MURPHY, 2012) defines machine learning as a set of methods to automatically detect and uncover patterns in data in order to perform decisions and predictions in new data. Therefore, machine learning methods are largely used in several areas as data mining (CURHAN; PENTLAND, 2007; EAGLE; PENTLAND, 2009), interaction (SATO; POUPYREV; HARRISON, 2012; SHOTTON et al., 2011) and specially computer vision (TORKI; ELGAMMAL, 2011; FENZI et al., 2013; HARA; CHELLAPPA, 2014).

Visual data can bring rich information of the scene, as seen in section 2.5, but also they can be highly complex(CRIMINISI; SHOTTON, 2013). Problems such as face recognition, which can be processed by human brain easily (FARAH, 1996; GAUTHIER et al., 2000), can be quite complex. However, with use of large supervised data set of human faces and machine learning methods, this recognition can be done with good accuracy (VIOLA; JONES, 2004). Moreover, problems of identification of numbers and words, finding human of object poses, and which type of objects is inside image data also can be solved by applying machine learning, illustrating the power of machine learning with visual data (MURPHY, 2012).

These recognition problems based on supervised training can be defined as a classification problem in machine learning, where the output of the machine is categorical or nominal (discrete and finite). Another type of problem, also solved based on supervised 
data, are the regression problems, where the output data is real valued (continuous space). These solutions can be used in problem such as pose estimation (PEPIK et al., 2012; GU; REN, 2010; HARA; CHELLAPPA, 2014; TORKI; ELGAMMAL, 2011)

Several methods of learning and prediction can be used for both problems, such as $k$-nearest neighbors $\left(k\right.$-NN), Expectation Maximization (EM), $l_{1}$ regularization, neural networks, supported vector machines (SVM), decision trees and decision forest, between others methods (MURPHY, 2012; CRIMINISI; SHOTTON, 2013).

\subsubsection{Decision Forest}

One special category of method of machine learning, that we explore in this work, are the decision forest (CRIMINISI; SHOTTON, 2013). However, in order to explain the decision forest concept, we first need to explain the idea of decision trees.

Decision trees (CRIMINISI; SHOTTON, 2013; MURPHY, 2012) are machine learning methods based on binary tree structures in order to perform classification/regression, based on multidimensional supervised observations. Each node of the decision tree contains a cut in the hyperspace of features, which can be a hyperplane or a hypercurve, to separate the groups of observations.

The decision itself is done by searching through the tree, and for each node a decision is made. This is repeated until a leaf node is found, which contains the possible returning value. Once only consecutive binary comparison are made at each node in the decision itself, decision trees process can reach good computation performance.

However, in the training stage, finding all the best separation for each node in the hyperspace can be computationally heavy. Moreover, reaching good accuracy can be hard for a single decision criteria (single tree). In order to address those problems, the decision forest approach uses several decision tree as weak learners.

By training several trees with different settings of observation, the training can be processed with less computation time. However, this makes each tree less accurate. This problem is avoided by combining the results returned by several trees. Those results are combined as

$$
P\left(\mathbf{T}_{o b j} \mid \mathbf{v}\right)=\frac{1}{Z} \prod_{i} P_{i}\left(\mathbf{T}_{o b j} \mid \mathbf{v}\right)
$$

where, each $P_{i}\left(\mathbf{T}_{o b j} \mid \mathbf{v}\right)$ are the probabilities returned by each tree. Those result combination can generates a faster training process, with more accurate result.

Also, once each tree is independent from each other, all the results can be processed in parallel, and then combined in the final process. This parallel process makes the decision forest have good performance, and suitable for real-time applications (SHOTTON et al., 2011; SHOTTON et al., 2013; CRIMINISI; SHOTTON, 2013). 


\subsection{Chapter Conclusions}

In this chapter, we have presented definitions used along this work. Some of methods used in this work are also presented with more detail. In the next chapter we present a study related to estimation of different objects poses. 


\section{Related Work}

In this chapter we present works in the literature related to estimation of object poses. Initially, methods of pose estimation of rigid objects based on RGB-D and RGB cameras, in sections 3.1 and 3.2 respectively, are presented. After that, we present works that do not rely on conventional global point information approaches, and focus on pose estimation of objects based on local information and machine learning, in section 3.3.

Then, we present works that focus on pose estimation of non-rigid objects, in section 3.4. In section 3.5, we discuss the similarities and differences, as well as, in what points our work is novel, when compared to all the works presented in this chapter. Finally, in section 3.6 we show our conclusions related to literature review.

\subsection{Rigid Object Pose Estimation based on RGB-D information}

RGB-D cameras became more accessible over the past years, becoming available as off the shelf products. As presented in section 2.5. These RGB-D cameras can bring valuable spatial information for object and camera tracking methods.

Several works for object/camera tracking based on these RGB-D spatial information find the object position by the correlation of the observed information with known information, for rigid objects pose estimation. Once the RGB-D frame contains depth information, every point observed points $\mathbf{p}^{c}$ are in $3 \mathrm{D}$ space $\left(\mathbf{p}^{c} \in \mathbb{R}^{3}\right)$. Thus, the camera rigid transformation $\mathbf{T}_{o b j}$ can be found directly as

$$
\mathbf{p}^{c}=\mathbf{T}_{o b j} \mathbf{p}^{o}
$$

and $\mathbf{p}^{o}$ are the known points of the object. This basic relation of the observed points and known points are the base for several works of pose estimation of rigid objects based on RGB-D information.

By equation (3.1), the object transformation can be fully solved using the Kabasch algorithm (KABSCH, 1976), which is based on singular value decomposition (SVD) (PRESS et al., 2007) of the rotation matrix. Works such as (CHOI; CHRISTENSEN, 2013) and (LEE; PARK; WOO, 2011) applies this solution in order to find the full object pose with feature points matching.

Choi e Christensen (2013) apply this solution with particle filter approach in order to obtain better accuracy of the final object pose. Here, the observation are calculated by the Kabsch method using the depth map point information; and the final particle likelihoods are calculated using information of object appearance, normal and position. 
On the other hand, Lee, Park e Woo (2011) combine this SVD based solution with templates matching method, using gradient values in RGB and depth images. This solution enables (LEE; PARK; WOO, 2011) to estimate both textured and non-textured objects poses. Note that, both (CHOI; CHRISTENSEN, 2013) and (LEE; PARK; WOO, 2011), use the Kabsch method with point positions in $\mathbb{R}^{3}$; although they also use other information for result refinement.

In contrast, another approach is taken in (CHOI; CHRISTENSEN, 2012). Unlike other works, color $c$, position $p$, normal $n$ and a secondary vector information $v$ are also applied, other than only the position information. The object pose is estimated by aligning the observed $\left(c^{w}, p^{w}, n^{w}, v^{w}\right)$ with the pre-stored $\left(c^{o}, p^{o}, n^{o}, v^{o}\right)$.

Marcon et al. (2012) also gather more local information of the surface, in order to apply in the feature points matching problem. Here, the principal component analysis (PCA) (JOLLIFFE, 2002) is used in order to extract normal vectors of the surface, and then, extracting planar regions of the RGB-D frame. Based on these planar regions, rectified images can be used to process matching of feature points with affine invariance.

Furthermore, Papazov e Burschka (2011) uses normal and vector between two points in order to process a fast pose estimation, based on RANSAC methods. Although, only depth information are used in this work.

Works such as (LIMA et al., 2012; PARK; LEPETIT; WOO, 2011) explores as additional information, $2 \mathrm{D}$ edges deformation information in order to estimate the poses of objects. Those 2D edges information not only can be used as a second observation to improve accuracy of the object (PARK; LEPETIT; WOO, 2011), but also, its deformation itself can gives prior knowledge of the object pose (LIMA et al., 2012).

Beside the works presented above, other authors focus on camera pose estimation and scene mapping based on the RGB-D frames. These works are mainly called simultaneous location and mapping (SLAM) works (KLEIN; MURRAY, 2007). Although they do not focus on object tracking for AR, camera pose estimation methods can be directly applied to object tracking.

Works such as (NEWCOMBE et al., 2011; WHELAN et al., 2013; HENRY et al., 2012; LEE; NGUYEN, 2014) achieve the camera pose estimation based on iterative closest point (ICP) methods (BESL; MCKAY, 1992). ICP methods take an approach to iteratively refining the pose of the desired model to the observed data, by finding the closest points between observed and desired model.

Although ICP based methods can give accurate results, they also has the disadvantage to depend on the proximity of the initial poses. Otherwise, being able to fall in wrong convergence at incorrect local minimums (HENRY et al., 2012). In order to avoid this problem and increase the computational speed, (HENRY et al., 2012) initially process 
a fast, feature point based RANSAC (FISCHLER; BOLLES, 1981) pose estimation with a model similar to equation (3.1). This estimation is, then, refined by ICP method. RANSAC based methods has the advantage of increasing the computational speed, and making the result robust to outliers (FISCHLER; BOLLES, 1981).

Use of RANSAC pose estimation with equation (3.1), can also be found in works such as (TAGUCHI et al., 2013) and (LEE; NGUYEN, 2014). Taguchi et al. (2013) applies the RANSAC methods to points and planes reconstructed from the observations. RGB information also can be directly used in the ICP cost function (WHELAN et al., 2013), increasing the dimensionality of the cost function from $\mathbb{R}^{3}$ to $\mathbb{R}^{6}$. Thus so, increasing the accuracy of the pose alignment.

Finally, Lee e Nguyen (2014) explores the use of RANSAC based initial pose alignment with ICP methods, but also gathering more local information. Here, authors also extract local normal vector from the surface, in order to be added in the ICP cost function. Creating a better point-to-plane error estimation between observed point information and reconstructed mesh model.

We show in table 1, the comparison between the works presented in this section. First we present the information used in the whole work (second column), and in the pose estimation process itself (third column); then, the method applied for the pose estimation is presented (fifth column). Finally, we also present if the work can obtain local pose from the input (forth column). 
Table 1 - Comparison of works based on RGB-D cameras for rigid object pose estimation.

\begin{tabular}{|c|c|c|c|c|c|c|c|c|c|c|c|c|}
\hline 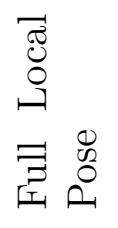 & $\stackrel{\circ}{Z}$ & $\stackrel{\circ}{z}$ & $\stackrel{\circ}{z}$ & $\stackrel{\circ}{Z}$ & $\stackrel{\circ}{Z}$ & $\stackrel{\circ}{\not}$ & $\stackrel{\circ}{Z}$ & $\stackrel{\circ}{z}$ & $\stackrel{\circ}{\not}$ & $\stackrel{\circ}{\not}$ & $\stackrel{\circ}{\not}$ & $\stackrel{0}{Z}$ \\
\hline 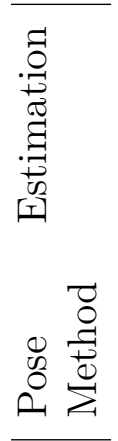 & 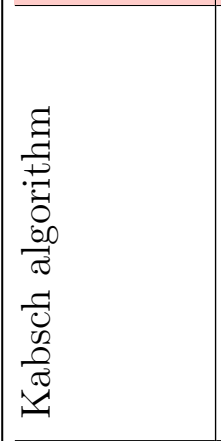 & 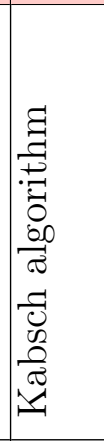 & 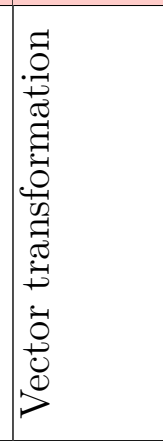 & 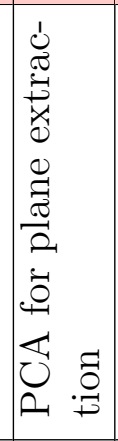 & 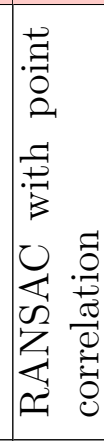 & 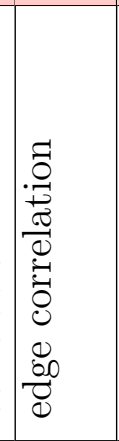 & 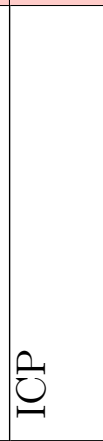 & 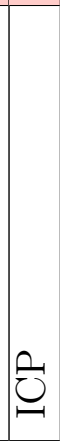 & 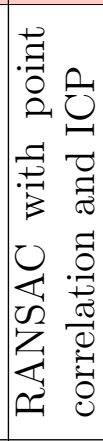 & 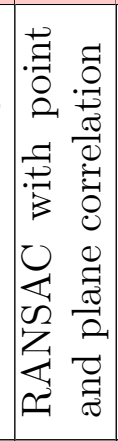 & $\underset{-1}{0}$ & $\underset{-1}{\circlearrowright}$ \\
\hline 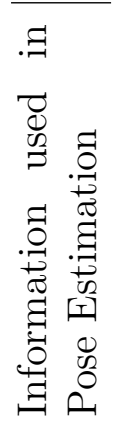 & 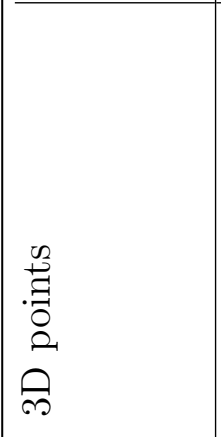 & 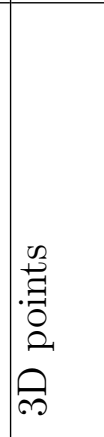 & 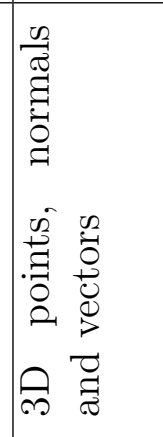 & & 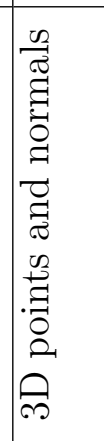 & 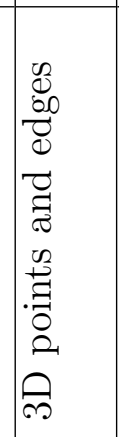 & $\begin{array}{l}0 \\
0 \\
00 \\
0 \\
0 \\
0 \\
0 \\
01 \\
0 \\
0 \\
.0 \\
0 \\
0 \\
2 \\
\infty\end{array}$ & 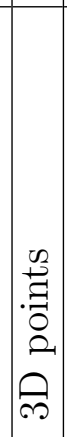 & 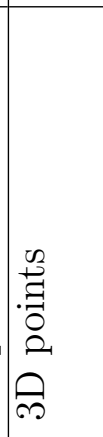 & 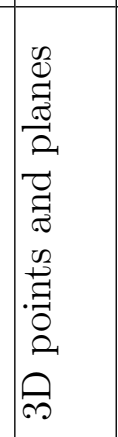 & 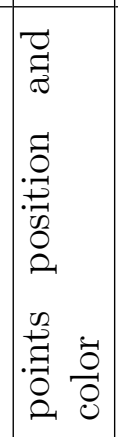 & 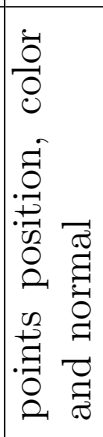 \\
\hline 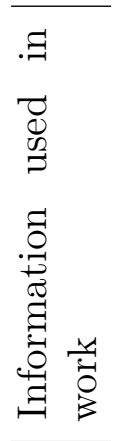 & 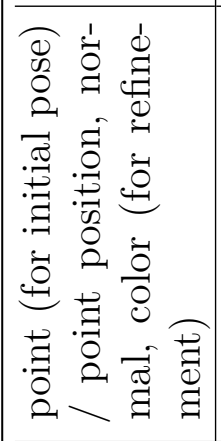 & 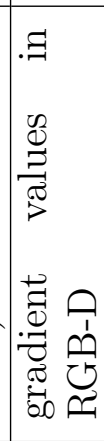 & 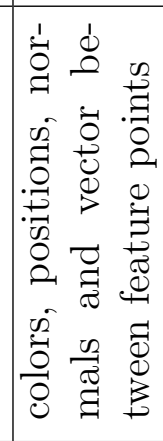 & 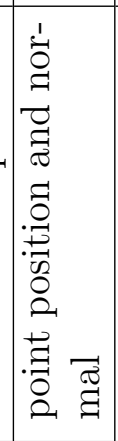 & 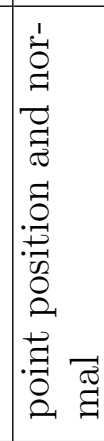 & 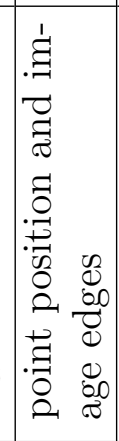 & 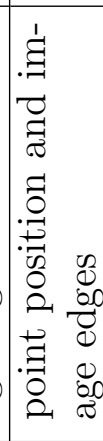 & 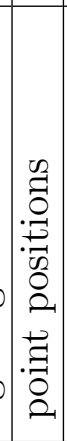 & 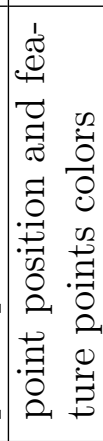 & 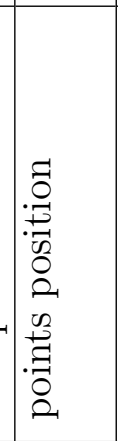 & 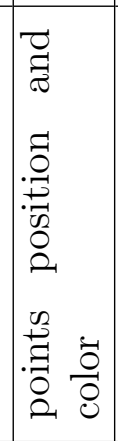 & 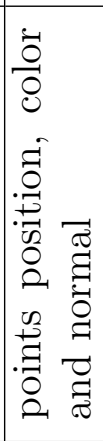 \\
\hline 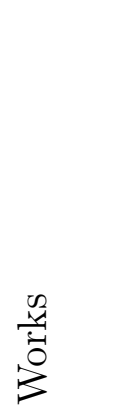 & 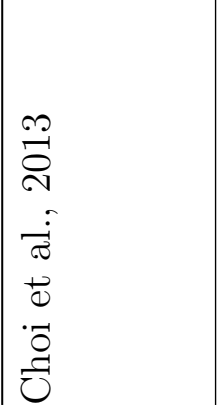 & 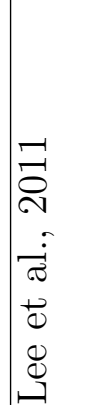 & 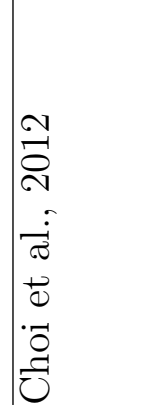 & 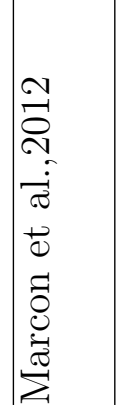 & 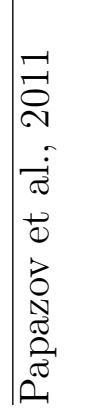 & 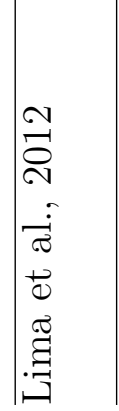 & 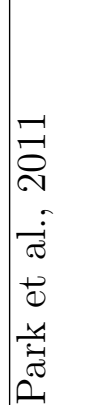 & 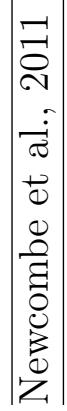 & 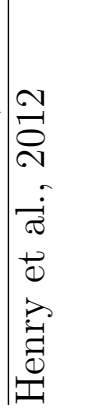 & 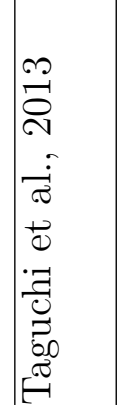 & 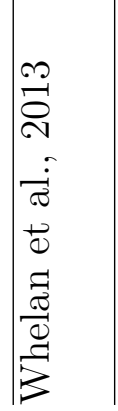 & 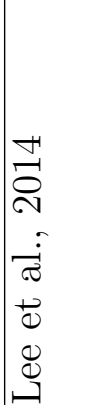 \\
\hline
\end{tabular}

We can observe, from this table, that none of the presented works can fully provide the local pose, although several are based on local information. Additionally, normal and point position are not sufficient in order to fully estimate the local pose; once orientation 
of the local feature is still not solvable. Despite (CHOI; CHRISTENSEN, 2012) does not apply its method for local information, it is the only method that could be directly applied to calculate the local poses.

Thus, from this section, we can observe that:

- Several works of camera/object pose estimation using RGB-D information simplify the observed object/scene information to simple points in space;

- Some of the works gather more local information in order to increase the computational speed or accuracy;

- None of works presented in this section fully achieve local pose of feature points.

\subsection{Rigid Object Pose Estimation based on RGB information}

In this section we presents works in literature that also focus on pose estimation, though, based only on RGB image information. However, RGB information lacks on full spatial information, unlike RGB-D images. Therefore, the several approaches in literature uses information such as 2D points and their spatial relationship (FISCHLER; BOLLES, 1981; LI; XU, 2011), edges (COSTA; SHAPIRO, 2000; HOLZER et al., 2009; VACCHETTI; LEPETIT; FUA, 2004) or edgels (WERNECK; COSTA, 2013; LOESCH et al., 2015) of the object.

Vacchetti, Lepetit e Fua (2004) present an approach to camera pose estimation based on edges and interest points. With this combined approach, authors can generate better accuracy and pose estimation even for textured and non-textured objects. Holzer et al. (2009), on the other hand, presents a object pose estimation based on edges, distance transformations and machine learning approach, also obtaining 6 DoF (degrees of freedom) texture-less object poses.

Werneck e Costa (2013) takes another approach for camera orientation estimation and uses edgels instead of full edges. Edgels (or edglets (DAMEN et al., 2012)) are short strain segments represented by its center and edge orientation. This information brings compromise between speed and accuracy for its extraction and camera rotation estimation. Loesch et al. (2015) also explore the use of edglets for 6 DoF object pose estimation. Thus, edge and edgel based approaches can generate pose estimations with good accuracy and even in real-time, specially for texture-less objects.

\subsubsection{Solve Perspective to $n$ Points Problem}

Despite the variety of different approaches for pose estimation of objects using RGB information, such as presented above, the approach that is mostly focus in several 
works are the approaches based on the perspective to $n$ points $(\mathrm{PnP})$ problem solvers (FISCHLER; BOLLES, 1981). Pose estimation approaches based on this approach shows accurate and precise results, and being largely used and studied in literature.

In these approaches, the problem is conventionally defined roughly as, given $n$ points observed in a perspective $2 \mathrm{D}$ projection of known $3 \mathrm{D}$ points, how we can retrieve the final 3D transformation or 3D data of these points. Thus, making the pose estimation problem a correlation problem between observed points in a RGB frame with the known $3 \mathrm{D}$ points of the object.

In more details, this problem can be defined in 2 different ways (WU; HU, 2006). The first way is defining this problem as (FISCHLER; BOLLES, 1981):

Given the relative spatial location of $n$ points $\mathbf{p}_{i}, i=1, \ldots, n$, and given the angle $\angle \mathbf{p}_{i} \mathbf{O} \mathbf{p}_{j}$ to every pair of these points from the perspective center $\mathbf{O}$ (the camera's optical center), find the lengths $x_{i}=\left|\mathbf{O} \mathbf{p}_{i}\right|$ of the line segments joining the perspective center to each of these points.

This definition (denominated as Definition1 in this work) conditions are illustrated in figure 8. From this figure and Definition1 itself, we can obtain the constraint equation as

$$
x_{i}^{2}+x_{j}^{2}-2 x_{i} x_{j} \cos \theta_{i j}=d_{i j}^{2}, \quad i \neq j
$$

where, $x_{i}$ and $x_{j}$ are the variables; in this case, the distance of the points $\mathbf{p}_{i}$ and $\mathbf{p}_{j}$ to the optics center; $\theta_{i j}=\angle \mathbf{p}_{i} \mathbf{O} \mathbf{p}_{j}$ is the angle formed by the optics center and the two points; and $d_{i j}=\left|\mathbf{p}_{i} \mathbf{p}_{j}\right|$ is the distance between points $\mathbf{p}_{i}$ and $\mathbf{p}_{j}$.

Figure 8-Definition1, 2-point constrain.

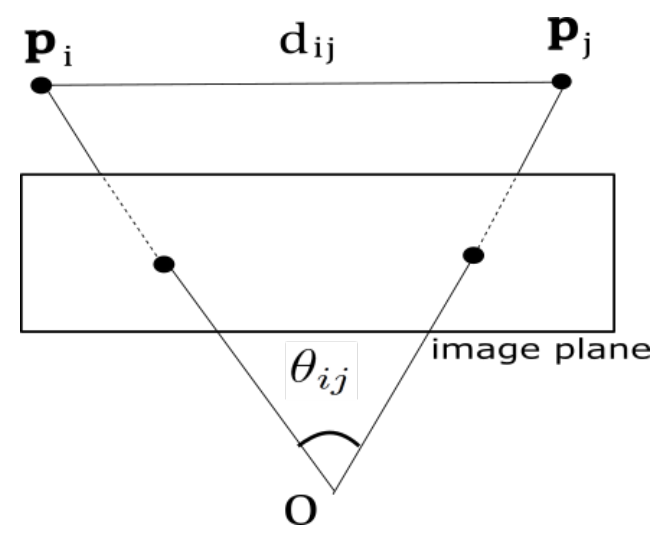

Source: Author, based on (LI; XU; XIE, 2012).

Note that this equation is based on a 2-point constrain, $\mathbf{p}_{i}$ and $\mathbf{p}_{j}$; and also that this equation cannot be computed by itself, since it has two unknown variables. However, 
when we consider a third point $\mathbf{p}_{k}$, we can obtain the 3-points constrain, such that:

$$
\left\{\begin{array}{l}
x_{i}^{2}+x_{j}^{2}-2 x_{i} x_{j} \cos \theta_{i j}=d_{i j}^{2}, \\
x_{i}^{2}+x_{k}^{2}-2 x_{i} x_{k} \cos \theta_{i k}=d_{i k}^{2} \\
x_{j}^{2}+x_{k}^{2}-2 x_{j} x_{k} \cos \theta_{j k}=d_{j k}^{2}
\end{array}\right.
$$

, with three unknown variables $x_{i}, x_{j}$ and $x_{k}$. This equation system can be converted into a forth order polynomial:

$$
f(x)=a x^{4}+b x^{3}+c x^{2}+d x+e=0 .
$$

This 3-point constrain, also known as P3P problem (LI; XU, 2011), can give us the depth of $\mathbf{p}_{i}, \mathbf{p}_{j}, \mathbf{p}_{k}$ when the polynomial (3.4) and (3.3) are solved. However, this polynomial can contain 4 solutions, when the 3 points are non-collinear, as pointed in works such as (LI; XU; XIE, 2012; LI; XU, 2011; WU; HU, 2006), thus the true object pose can not be determined in this point. Figure 9 illustrates the possible solutions that this equation can give.

Figure 9 - The 4 different solutions for the equation (3.4). All the 4 triangles, $\left(\mathbf{p}_{i}, \mathbf{p}_{j}, \mathbf{p}_{k}\right)$, $\left(\mathbf{p}_{i}^{\prime}, \mathbf{p}_{j}, \mathbf{p}_{k}\right),\left(\mathbf{p}_{i}, \mathbf{p}_{j}^{\prime}, \mathbf{p}_{k}\right),\left(\mathbf{p}_{i}, \mathbf{p}_{j}, \mathbf{p}_{k}^{\prime}\right)$, can be solutions.
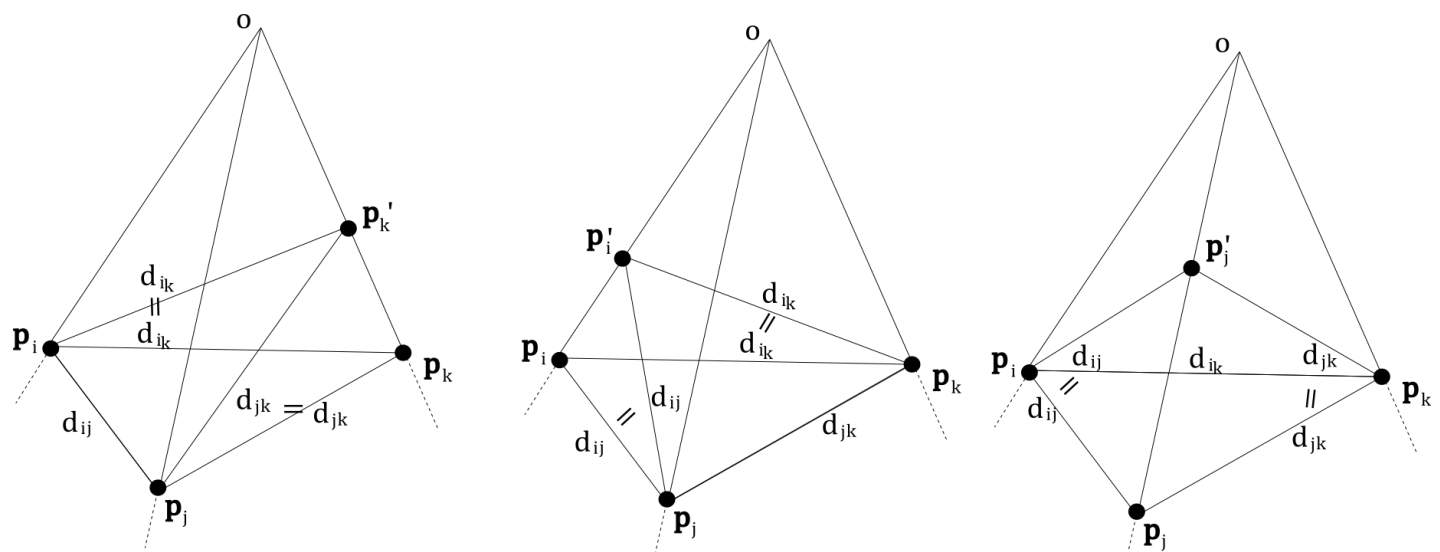

Source: Author, based on (FISCHLER; BOLLES, 1981).

In order determinate a unique solution for the object pose, more points $\mathbf{p}_{l}$ needs to be used (such as in P4P problem (GRAFAREND; SHAN, 1997; BUJNAK; KUKELOVA; PAJDLA, 2008)). As more general case, where $n$ points are used in the problem, as described in Definition1, the PnP problem turns to a error minimization problem, once, more observations are collected than necessary to obtain the unique solution. In order to solve those polynomial equations mathematical tools such as linearization techniques or by the eigenvalue method (PRESS et al., 2007) can be applied. However, they often fall in a seventh order polynomial solving problem (once that the forth order polynomial error minimum value is calculated by taking the derivation of the polynomial, becoming a seventh order polynomial solving problem). 
By using these constrains, which gives the correlation between known 3D points its observed projections in the image plane, the 3D information of points in space coordinates can be determined. Several works in literature are based on these constrains and rigid object characteristics, such as presented in section 2.1, in order to estimate known objects poses.

On the other hand, the PnP problem can also be defined as (HORAUD et al., 1989):

Given $n$ points $\mathbf{p}_{i}, i=1, \ldots, n$ with known coordinates in an object centred frame, and their corresponding projection $p_{i}^{I}, i=1, \ldots, n$ onto a image plane, and given the intrinsic camera parameters $\mathbf{K}$, find the transformation (a rotation matrix $\mathbf{R}$ and a translation vector $\mathbf{t}$ ) between the object frame and the camera frame.

In this definition (denominated as Definition2 in this work) the applied constraint equation is

$$
p_{i}^{I} \approx \mathbf{K}(\mathbf{R}, \mathbf{t}) \mathbf{p}_{i}^{H},
$$

where the rotation matrix $\mathbf{R}$ and translation vector $\mathbf{t}$ are the unknowns, and $\mathbf{K}, \mathbf{M}_{i}$ and $\mathbf{m}_{i}$ are known parameters.

From (3.5), and by projecting the image position $p_{i}^{I}$ in pixels to the image plane in space $\mathbf{p}_{i}^{I}=\left[u_{i} v_{i} 1\right]^{\top}$ using $\mathbf{K}$, we can obtain the equations

$$
u_{i}=\frac{\mathbf{r}_{1}^{\top} \mathbf{p}_{i}^{W}+t_{x}}{\mathbf{r}_{3}^{\top} \mathbf{p}_{i}^{W}+t_{z}}, \quad v_{i}=\frac{\mathbf{r}_{2}^{\top} \mathbf{p}_{i}^{W}+t_{y}}{\mathbf{r}_{3}^{\top} \mathbf{p}_{i}^{W}+t_{z}}
$$

, where $\mathbf{R}=\left[\mathbf{r}_{1}^{\top} \mathbf{r}_{2}^{\top} \mathbf{r}_{3}^{\top}\right]^{\top}$ and $\mathbf{t}=\left[t_{x} t_{y} t_{z}\right]$. Equation (3.6) also can be rewrite as

$$
\mathbf{p}_{i}^{I}=\frac{1}{\mathbf{r}_{3}^{\top} \mathbf{p}_{i}^{W}+t_{z}}\left(\mathbf{R}_{i}^{W}+\mathbf{t}\right)
$$

, this equation is known as collinearity equation in photogrammetry literature.

$\mathrm{Wu}$ e $\mathrm{Hu}(2006)$ analyses the relation ship between both definitions. As in (HU; WU, 2002), Wu e Hu (2006) show that both definition, although similar, are not equivalent. The authors also show that the major difference between both definitions are in the number of possible solutions in the case o $\mathrm{P} 4 \mathrm{P}$ problem. Under P3P and $\mathrm{P} 5 \mathrm{P}$ constrains, both definitions has the same number of solutions.

The pose estimation solutions also can be classified in two different types, the iterative and non-iterative methods. Iterative approaches conventionally uses equation (3.6) as base line and minimize the squared errors objective function

$$
\sum_{i=1}^{n}\left[\left(u_{i}-\frac{\mathbf{r}_{1}^{\top} \mathbf{p}_{i}^{w}+t_{x}}{\mathbf{r}_{3}^{\top} \mathbf{p}_{i}^{w}+t_{z}}\right)^{2}+\left(v_{i}-\frac{\mathbf{r}_{2}^{\top} \mathbf{p}_{i}^{w}+t_{y}}{\mathbf{r}_{3}^{\top} \mathbf{p}_{i}^{w}+t_{z}}\right)^{2}\right]
$$


with different iterative optimization methods such as Gauss-Newton () or LevenbergMarquardt ().

Furthermore, Lu, Hager e Mjolsness (2000) present a fast and accurate iterative method based on Definition2. Authors point that a minimization by equation (3.8) is actually over image-space collinearity. Therefore, in some conditions, this image-space minimization can converge slowly or even fail to converge. (LU; HAGER; MJOLSNESS, 2000) avoid these problem based on a more direct object space error function, and approaching as an absolute orientation problem.

(GARRO; CROSILLA; FUSIELLO, 2012) offers an alternative minimization methods based on algebraic error defined in the image space and defining the PnP as an anisotropic orthogonal procustes problem. However, these iterative methods has the risk of getting trapped in local minimums, providing poor results; and several of them, are highly dependent of the initialization pose of the iterative loop, as in (LU; HAGER; MJOLSNESS, 2000).

On the other hand, the solutions to (3.4) are calculated directly in case of noniterative approaches, making those solutions more efficient than iterative ones (LI; XU; XIE, 2012; LEPETIT; MORENO-NOGUER; FUA, 2009). Even (LU; HAGER; MJOLSNESS, 2000) method, that is fast in comparison to other iterative methods, is slow when compared to non-iterative solutions (LEPETIT; MORENO-NOGUER; FUA, 2009).

Fischler e Bolles (1981) is one of the first to define the PnP problem(Definition1); and solve it by scaling down the $n$ points problem to subset of 3 or 4 points. Those constrains are solved by closed form solutions (REES, 1922) or iterative techniques. In order to obtain the global consistency of the results, authors also introduce the RANSAC paradigm.

Here, the estimated result of P3P problem, of a randomly selected set of 3-points, is applied to a subset of the observed points, and the re-projected error is calculated. If a certain percentage of the points in this subset has smaller error then a predefined threshold value, than accept the local P3P solution. In case the percentage is smaller then expected, the P3P problem is re-solved with other randomly selected set of 3-points and the re-projection error percentage is calculated again, until the percentage is accepted.

This RANSAC paradigm can also be used in several other cases; and, as pointed by authors, it has the advantage of being more robust in data sets that contains a significant percentage of gross errors. This is achieved, once part of the data is randomly selected, and the consensus in this data set is calculated. Even, when the points selected to solve the problem contains gross errors, it is filtered by the recalculation approach in order to achieve a acceptable consensus. We also apply the RANSAC paradigm in our work in different conditions (sections 7.3.1 and 8.4). 
Differently, Lepetit, Moreno-Noguer e Fua (2009) presents the EPnP, a methods with $O(n)$, where the object world coordinate are represented by a weighted sum of control points. (LEPETIT; MORENO-NOGUER; FUA, 2009) first defines a set of four control points $\mathbf{c}_{j}, j=1, \ldots, 4$. In practice, authors points that stability can increase if the centroid of the object is selected as one control point, and the other three points are selected such as form a basis aligned with the principal directions.

All the object points are represented as a weighted sum of the control points such as

$$
\mathbf{p}_{i}^{w}=\sum_{j=1}^{4} \alpha_{i j} \mathbf{c}_{j}^{w}, \quad \text { with } \quad \sum_{j=1}^{4} \alpha_{i j}=1,
$$

where, $\alpha_{i j}$ are the homogeneous baricentric coordinates. Once the object is considered to have a rigid transformation in relation with to camera coordinates, the relation of $\alpha_{i j}$ does not changes. Thus in camera coordinates, this relationship can be write as

$$
\mathbf{p}_{i}^{c}=\sum_{j=1}^{4} \alpha_{i j} \mathbf{c}_{j}^{c},
$$

where, $\mathbf{c}_{j}^{c}$ are the transformed coordinate system to the camera coordinate system, and are the unknowns to be found.

Combining (3.10) with projection matrix, we can obtain the equation

$$
p_{i}^{I}=\left[\begin{array}{c}
x_{i}^{I} \\
y_{i}^{I} \\
1
\end{array}\right]=\mathbf{K}\left(\frac{\sum_{j=1}^{4} \alpha_{i j} \mathbf{c}_{j}^{c}}{\sum_{j=1}^{4} \alpha_{i j} z_{j}^{c}}\right),
$$

where $\mathbf{c}_{j}^{c}=\left[x_{j}^{c}, y_{j}^{c}, z_{j}^{c}\right]$, are the control points values. By concatenating and rearranging the equations of (3.11), we obtain $\mathbf{M x}=0$, where $x=\left[c_{1}^{c^{\top}}, c_{2}^{c^{\top}}, c_{3}^{c^{\top}}, c_{4}^{c^{\top}}\right]^{\top}$, and $\mathbf{M}$ a $2 n \times 12$ matrix. The solution of $\mathbf{x}$, as pointed by authors, belongs to the null space of $\mathbf{M}$. Therefore can be expressed and as

$$
\mathbf{x}=\sum_{i=1}^{N} \beta_{i} \mathbf{v}_{i}
$$

where $\mathbf{v}_{i}$ are the column of the right-singular vectors of $\mathbf{M}$, and can be found as the null eigenvectors of matrx $\mathbf{M}^{\top} \mathbf{M}$. By finding $\mathbf{v}$ and $\beta_{\mathbf{i}}$ based on relinearization technique (KIPNIS; SHAMIR, 1999), it is possible to retrieve $\mathbf{x}$, that contains the object observed coordinates.

Authors also suggest, in the paper, that methods of gauss-newton optimization can be applied in order to obtain further improvement in the results accuracy, by using the results obtained above as initialization parameter of the iterative algorithm (named as EPnP-GN). The result of EPnP and EPnP-GN are one of the state-of-art results in literature, even in accuracy and computational time. Thus its results are often used in performance comparison in other works. 
$\mathrm{Li}$, Xu e Xie (2012) present the robust non-iterative PnP solution (RPnP) approach, also with $O(n)$; which improves the accuracy based on Definition1, and calculating a rotation axis as reference coordinate system. Initially, a rotation axis is selected, by finding the longest vector $\overrightarrow{\mathbf{p}_{i 0}^{w} \mathbf{p}_{j 0}^{w}}$ formed by a pair of observed points $\mathbf{p}_{i}^{w}$.

Then, a coordinate system $O_{a} X_{a} Y_{a} Z_{a}$ is chosen such that the origin $O_{a}$ is placed in the middle of $\overrightarrow{\mathbf{p}_{i 0}^{w} \mathbf{p}_{j 0}^{w}}$ and $Z_{a}$ axis direction is the same as $\overrightarrow{\mathbf{p}_{i 0}^{w} \mathbf{p}_{j 0}^{w}}$. All the points within $\mathbf{p}_{i}^{w}$ are now represented in this new coordinate system $O_{a} X_{a} Y_{a} Z_{a}$. And now, the transformation between the camera coordinate system to object coordinate system can be represented as a rotation axis $Z_{a}$, rotation angle $\alpha$ around $Z_{a}$, and a translation vector between the origins.

By applying the 3 point constrain equation (3.4) of Definition1 in all the subsets formed by three points group $\left\{\mathbf{p}_{i 0}^{w} \mathbf{p}_{j 0} \mathbf{p}_{k}, k \neq i 0, k \neq j 0\right\}$, authors can obtain the 3D position of $\mathbf{p}_{i 0}^{w}$ and $\mathbf{p}_{j 0}^{w}$. For that purpose, the solution with minimal least square residual error $F=\sum_{i}(x)^{2}$ needs to be found. This is done by finding the roots of its derivative

$$
F^{\prime}=\sum_{i} f(x) f_{i}^{\prime}(x)
$$

a seventh order polynomial, solved by eigenvalue methods (PRESS et al., 2007).

After finding the rotation axis, the rotation angle and translation vector is calculated by transforming all the points as a rotation in $Z_{a}$ axis, and correlating with the projected points in the observed image. This is done by also calculating a linear equation system formed the the projections, using Singular Value Decomposition (SVD) techniques (PRESS et al., 2007).

Finally, Zheng et al. (2013) present the OPnP approach, also with $O(n)$ based on quaternion representation (AKENINE-MOLLER; MOLLER; HAINES, 2008) and a blind Gröbner solver (KUKELOVA; BUJNAK; PAJDLA, 2008), also based on Definition2. As pointed by authors, their approach can achieve better accuracy even compared to state-of-art iterative methods. They achieve this, by reformulating the $\mathrm{PnP}$ problem as an error minimization problem. First the observed points are represented as

$$
d_{i} \mathbf{p}_{i}^{p I}=\mathbf{R} \mathbf{p}_{i}^{w}+\mathbf{t}
$$

where $d_{i}$ is the depth of each observed point $\mathbf{p}_{i}^{w}$ projected on the image plane, represented as $p_{i}^{p I}=\left[u_{i}, v_{i}, 1\right]^{\top} ; \mathbf{t}$ is the translation vector; and $\mathbf{R}$ is the rotation matrix, represented as a non-unit quaternion

$$
\mathbf{R}=\frac{1}{s}\left[\begin{array}{ccc}
a^{2}+b^{2}-c^{2}-d^{2} & 2 b c-2 a d & 2 b d+2 a c \\
2 b c+2 a d & a^{2}-b^{2}+c^{2}-d^{2} & 2 c d-2 a b \\
2 b d-2 a c & 2 c d+2 a b & a^{2}-b^{2}-c^{2}+d^{2}
\end{array}\right]=\frac{1}{s}\left[\begin{array}{c}
\mathbf{r}_{1}^{\top} \\
\mathbf{r}_{2}^{\top} \\
\mathbf{r}_{3}^{\top}
\end{array}\right],
$$

and $a, b, c$ and $d$ are the unknown of the rotation matrix; and $s$ is the scale factor to make $\operatorname{det}(\mathbf{R})=1$. 
By combining (3.14) and (3.15), authors obtain the minimization function of the squared errors

$$
\underset{a, b, c, d, \hat{t}_{1}, \hat{t}_{2}}{\arg \min } \sum_{i=1}^{n}\left[\left(1+\mathbf{r}_{3}^{\top}\left(\mathbf{p}_{i}^{w}-\overline{\mathbf{p}}^{w}\right)\right) u_{i}-\mathbf{r}_{1}^{\top} \mathbf{p}_{i}^{w}-\hat{t}_{1}\right]^{2}+\sum_{i=1}^{n}\left[\left(1+\mathbf{r}_{3}^{\top}\left(\mathbf{p}_{i}^{w}-\overline{\mathbf{p}}^{w}\right)\right) v_{i}-\mathbf{r}_{2}^{\top} \mathbf{p}_{i}^{w}-\hat{t}_{2}\right]^{2},
$$

where $\overline{\mathbf{p}}^{w}$ represents the centroid of the object points; $\left[\hat{t}_{1}, \hat{t}_{2}, \hat{t}_{3}\right]^{\top}=\frac{1}{d} \mathbf{t}$ and $d=\frac{1}{n} \sum_{i=1}^{n} d_{i}$.

Now, by solving the equation (3.16), authors can obtain the rotation and translation of the object. In order to solve this equation, they apply a blind Gröbner basis technique (KUKELOVA; BUJNAK; PAJDLA, 2008). Gröbner basis are a set of generators of the ideal, set of all polynomials that can be generated by the polynomials functions to be solved. According to (ZHENG et al., 2013), using the automatic generator in (KUKELOVA; BUJNAK; PAJDLA, 2008), the polynomial derived by (3.16) has at most 81 solutions and can be solved with good computational time. Their final results shows to have better accuracy and computational time than other state-of-art methods.

However, as presented in this section, specially by the equations $(3.8)(3.12)(3.13)(3.16)$, most of the works based on the PnP problem are dependent on non-linear polynomial solvers. This comes from the 3-points constrain at equation (3.7), showing that this inverse problem has the nature to be a complex, polynomial and minimal problem solving with several variables. As pointed by Kukelova, Bujnak e Pajdla (2008), finding such solutions with accuracy and computational efficiency is not trivial.

Also, most of the works approach this problem by a global error minimization problem in order to obtain better stability. Moreover, they, by definition (Definition1 and Definition2), simplify the pose estimation by considering that the only known information of the object and observed scene are the object points and its projection to the image plane. These facts leads to solutions that throw away valid information of the object, specially local information around feature points, which can be explored for pose estimation.

Thus, we can observe from this section that:

- Conventional solve-PnP based approaches, in most cases, rely on complex non-linear polynomial solving;

- Conventional solve-PnP based approaches also simplify the observed object information to simple points in space.

\subsection{Pose Estimation Based on Local Information and Machine Learning}

In sections 3.2 and 3.2, we have presented several related works on object pose estimation. Most of the works presented use global information directly in order to estimate 
objects poses. Therefore, interest points are simplified to single point position or edge directions information.

On the other hand, some works in literature have the focus on gathering more local information, in order to estimate the object spatial pose. One of the approaches is to apply machine learning over those local information. This can be achieved by training changes of observed structures of the object, such as texture appearances or position changes of known projected points, across different views. Those approaches have the advantage of avoiding the complex non-linear optimization solving. Instead that, delegating the problem to machines to solve the pose related to the observations.

Murase e Nayar (1993) present an approach which collect a set images of the object, and uses those images to find the object and its pose. In this work, Murase and Nayar first collect images of the object in different poses with one dimensional rotation and in different illumination conditions, using a turning table and a moving light source.

Then, each image is converted to a vector $x_{r, l}^{(p)}$, where $p$ represents the object number, $r$ the pose, and $l$ are the illumination conditions, read in a raster scan manner. Each pixel brightness value in the image are normalized and written in $x_{r, l}^{(p)}$. The sets of all the images, $X_{R, L}^{P}$, is then computed to find the eigenvectors of this image set space and diminishes the search hyperspace.

Finally, authors compute a hypersurface formed by the $X_{R, L}^{P}$ in the eigenspace formed by the eigenvectors; $g^{(p)}(r, l)$ for the object $p$, and $f^{(p)}(r, l)$, for the different poses and illuminations. This is done to filter noises across different views, and can be done, once, each near views of the object has small changes.

Now, for each new input image $y$ with a object, its pose and classification is calculated by finding the projection of $y$ into the eigenspaces, $z$ for the object hyperspace and, $z^{(p)}$ into the pose space. The the distances to the hypersurfaces are calculated as:

$$
\begin{array}{r}
d_{o}=\min _{(r, l)}=\left\|z-g^{(p)}(r, l)\right\| ; \\
d_{r, l}=\min _{(r, l)}=\left\|z^{(p)}-f^{(p)}(r, l)\right\| .
\end{array}
$$

By finding those minimal distances to each hyperspace, authors can find the values of object class, $p$, pose and illumination, $(r, l)$, of the new image.

He, Sigal e Sclaroff (2014) presents another method based on the LLC encoding method (WANG et al., 2010). HoG features (DALAL; TRIGGS, 2005) are used in order to train the observations as feature vectors. LLC method is based on features, such as SIFT, and processed with K-means and pooling methods to extract the visual words. He, Sigal e Sclaroff (2014) present a cascade discrete-continuous algorithm to learning those visual words using structural SVMs. As results, authors can estimate continuous object poses with 2 rotation axes, tilt and pan; and a discrete poses in 2 rotation axes for 3D objects. 
As (HE; SIGAL; SCLAROFF, 2014), several works uses HoG features (DALAL; TRIGGS, 2005) as features to be trained. Pepik et al. (2012) approach also is based on HoG features. Here, the authors add 3D displacement model at the latent variable structured SVM objective, in order to train the deformation of appearance caused by 3D perspective, for multiple parts of one object. The training itself is based on data, which contains tuples $I_{i}, y_{i}$; where $I_{i}$ are the images, and the output variable consisting of three parts $y_{i}=\left(y^{l}, y^{v}, y^{b}\right) . y^{l}$ contains the object class information, $y^{v}$, the rotation around one axis, and $y^{b}$, the $3 \mathrm{D}$ bounding box of the object.

Other works, such as (GU; REN, 2010; HARA; CHELLAPPA, 2014; SHIMIZU et al., 2015; ZHEN et al., 2015) also exploit HoG features as input for the training.Gu e Ren (2010) combines HoG with latent SVM (FELZENSZWALB et al., 2010) training.

On the other hand, Hara e Chellappa (2014) and Shimizu et al. (2015), both use HoG features in combination with regression forest (CRIMINISI; SHOTTON, 2013) in order to train the object poses, in one axis of rotation (HARA; CHELLAPPA, 2014) and in two axis of rotations (SHIMIZU et al., 2015). Finally, (ZHEN et al., 2015) combines multiscale HoG features with supervised manifold regularization training, similar to Murase eigenspace, achieving two axis of rotation estimation.

Once HoG features are based on the gradient of the pixel brightness in several blocks, they can train the changes of edges of the object according to several views. Making works that uses HoG features for training, such as (GU; REN, 2010; HARA; CHELLAPPA, 2014; SHIMIZU et al., 2015; HE; SIGAL; SCLAROFF, 2014), similar to the ones that uses edges to estimate objects poses (VACCHETTI; LEPETIT; FUA, 2004; HOLZER et al., 2009; WERNECK; COSTA, 2013). However, HoG features are not rotation and scale invariant (STEFANOU; ARGYROS, 2012). Although, there are variants that enables rotation and scale invariance (STEFANOU; ARGYROS, 2012), HoG features are not capable to enable full pose detection by itself.

Torki e Elgammal (2011) present a different approach based on a joint appearancespatial embedded space of feature points points with SVM learning. Here, each input image $k$ for training are represented as a set $X^{k}=\left\{\left(x_{0}^{k}, f_{0}^{k}\right), \ldots,\left(x_{n}^{k}, f_{n}^{k}\right)\right\}$, where $x_{n}^{k}$ denotes the feature points spatial location in the image; and $f_{n}^{k}$, its descriptor. Also, the output label to be trained are the pose in a single axis of rotation $v_{k}$ for each image.

This embedded joint space is non-linear. Thus, in order to achieve a joint featurespatial embedding space that satisfy the SVM learning constraints, authors also propose the object function

$$
\Phi(Y)=\sum_{k} \sum_{i, j}\left\|y_{i}^{k}-y_{j}^{k}\right\|^{2} \mathbf{S}_{i, j}^{k}+\lambda \sum_{p, q} \sum_{i, j}\left\|y_{i}^{p}-y_{j}^{q}\right\|^{2} \mathbf{U}_{i, j}^{p, q},
$$

where, $y_{i}^{k}$ are the feature-spatial embedded space position of $\left(x_{0}^{k}, f_{0}^{k}\right) . \mathbf{S}_{i, j}^{k}=K_{s}\left(x_{i}^{k}, x_{j}^{k}\right)$ is a weight matrix of the spatial kernels of the feature points positions, and $\mathbf{U}_{i, j}^{p \cdot q}=K_{s}\left(f_{i}^{p}, f_{j}^{q}\right)$, 
the weight matrix of the descriptor space kernels. By this objective function, (TORKI; ELGAMMAL, 2011) achieve the SVM training in the joint appearance and spatial changes of the whole object, in one rotation axis.

However, using location of feature points can fall to conditions that are dependent of scale and other cases unless explicitly avoided, as pointed by in (FENZI et al., 2013). Instead, Fenzi et al. (2013) take an approach based only on the feature points descriptors changes across the object poses.

In this work, the input for the training is modelled as $t^{i}=\left\{\left(f_{0}^{i}, \alpha_{0}^{i}\right), \ldots,\left(f_{n}^{i}, \alpha_{n}^{i}\right)\right\}$, where $t^{i}$ are the collections of the same 3D planar feature point information in different views. $f_{j}^{i}$ is the descriptor of this feature captured in a image with the object in a pose $\alpha_{j}^{i}$. Note that, the input data is the same feature point $t^{i}$ across different views $\left(\alpha_{0}^{i}, \ldots, \alpha_{n}^{i}\right)$ in this work; unlike other works, that model the input as sets of independent features observed across different views.

These information are clustered in individuals models to build a class representation of the object, by applying an approach based on dynamic time warping (SAKOE; CHIBA, 1978). This approach, well known in audio processing, is based in a dynamic programming method to calculate the similarity score; which is then, clustered using k-means method (MURPHY, 2012).

Then, the object pose and class are estimated by using a set of feature point descriptors $\mathcal{F}$, observed in a new frame. First, the class of each observed feature point is estimated by matching the points to the cluster model, creating the estimated class set $\mathcal{C}$. Then, the pose $\alpha^{*}$ is estimated by finding the probability

$$
\alpha^{*}=\operatorname{argmax}_{\alpha} p(\alpha \mid \mathcal{F}, \mathcal{C}) \approx \operatorname{argmax}_{\alpha} \sum_{r} p\left(f_{r}, c_{r} \mid \alpha\right) p(\alpha),
$$

where, $p\left(f_{r}, c_{r} \mid \alpha\right)$ is the probability of finding a feature $f_{r}$ in a cluster $c_{r}$ with a estimated $\alpha$. This $\alpha$ is estimated by the distance between the observed feature point descriptor with a trained statistical manifold modeling of the object pose space (MEI et al., 2011). Thus, the authors estimate the object class and pose of each feature point (i.e, the feature point local pose, for then obtaining the global object model and pose).

Works such as (SHOTTON et al., 2013; KRULL et al., 2015; BRACHMANN et al., 2014; TEJANI et al., 2014), although based on RGB-D cameras, also present object classification and full pose estimation based on machine lerning. Shotton et al. (2013) take an approach of camera pose estimation training based on random forest regression. 3D positions of a scene are trained as labels of the random forest. Features based on comparisons between pixel values (brightness in each color channel and depth) that surrounds certain pixel $p$ are used as input. These feature are defined as

$$
f_{\phi}^{r g b-d}(p)=I\left(p+\frac{\delta_{1}}{D(p)}, c 1\right)-I\left(p+\frac{\delta_{2}}{D(p)}, c 2\right) .
$$


Here $\delta$ indicates a 2D offset, $D(p)$ indicates a depth value and $I(p, c)$ indicates the brightness of the pixel in the channel $c$, which includes the depth channel. Each set of parameters $\delta_{1}, \delta_{2}, c 1, c 2$ returns a different value of feature that will be used in the training.

Using random forest training methods (CRIMINISI; SHOTTON, 2013), authors train the forest with several feature values for the camera positions regression. Then, the camera position is calculated by estimating several pixels of the input frame with the random forest and applying Kabsch algorithm (KABSCH, 1976). Finally, by combining RANSAC algorithm with the random forest regression, authors optimize the final returned value of the pose estimation.

Brachmann et al. (2014) take a similar approach for 6 DoF pose estimation of objects. In this work, the object class is also trained in order to detect several objects using a single decision forest. Using also a RANSAC based algorithm, authors estimates the object pose refining the possibilities of multiple points correlation between the observed RGB-D information with the returned object positions from the random forest.

Krull et al. (2015) also takes a similar approach to (BRACHMANN et al., 2014; SHOTTON et al., 2013). Here, authors combine particle filtering (ISARD; BLAKE, 1998) method with regression forest, in order to estimate the object poses using RGB-D frames. The random regression forest are trained to find discrete labels of the $3 \mathrm{D}$ position in object centered coordinates based on features calculated as in (3.20) and pixels values of the new frame, as in (BRACHMANN et al., 2014). Then, by applying the Kabsch algorithm (KABSCH, 1976) several estimations of object pose are extracted. These poses are refined by particle filtering algorithm.

Finally, Tejani et al. (2014) take a slightly different approach than taken at (BRACHMANN et al., 2014; SHOTTON et al., 2013; KRULL et al., 2015). Similar to other works, Tejani et al. (2014) also apply an random forest with the RANSAC method in order to estimate the object pose.

Yet, unlike previous works, (TEJANI et al., 2014) apply as training label

$$
\theta_{i}=\left(\theta_{x}, \theta_{y}, \theta_{z}, \theta_{\text {yaw }}, \theta_{\text {pitch }}, \theta_{\text {roll }}\right)
$$

Where $\left(\theta_{x}, \theta_{y}, \theta_{z}\right)$ are the $3 \mathrm{D}$ position to the object center and $\left(\theta_{\text {yaw }}, \theta_{\text {pitch }}, \theta_{\text {roll }}\right)$, the $3 \mathrm{D}$ Euler angles rotation. Therefore, authors trains directly the final object pose. Also, the features applied here are not only features calculated by (3.20), but gradient of the image and normal vector orientation.

However, note that all of RGB-D based works, although obtain the pose regression as output, uses the depth (and the image XY position) in order to estimate the object poses (or scene). This makes those approaches hard to directly use in RGB information.

Other than that, works such as (FENZI et al., 2015) explores linear programming 
methods for the pose estimation of video sequences. Also, works such as (ZHANG et al., 2015) explores the pose regression in $1 \mathrm{D}$ and $2 \mathrm{D}$ rotation by applying a gaussian process regression.

Although all of the presented works in this section explore the local information for pose estimation, instead of simplifying the object to a set of points; all the RGB based works does not fully achieve 6 DoF pose, nor full local rotation or pose. In table 2, we present the comparison of these works, showing how many degrees of freedom (DoF) the final estimation can provide (second column); if its input information is RGB or RGB-D (third column); and if the information used for regression and pose estimation are local or global (forth column).

We also present the input data used in the pose regression learning (fifth column); learning method applied in order to train the object pose (sixth column); and finally, the output of the learner (seventh column). We highlight each cell of the second to forth columns with red and green colors. Thus showing some characteristics, which will be discussed here. 
Table 2 - Comparison of works based on machine learning for object pose estimation

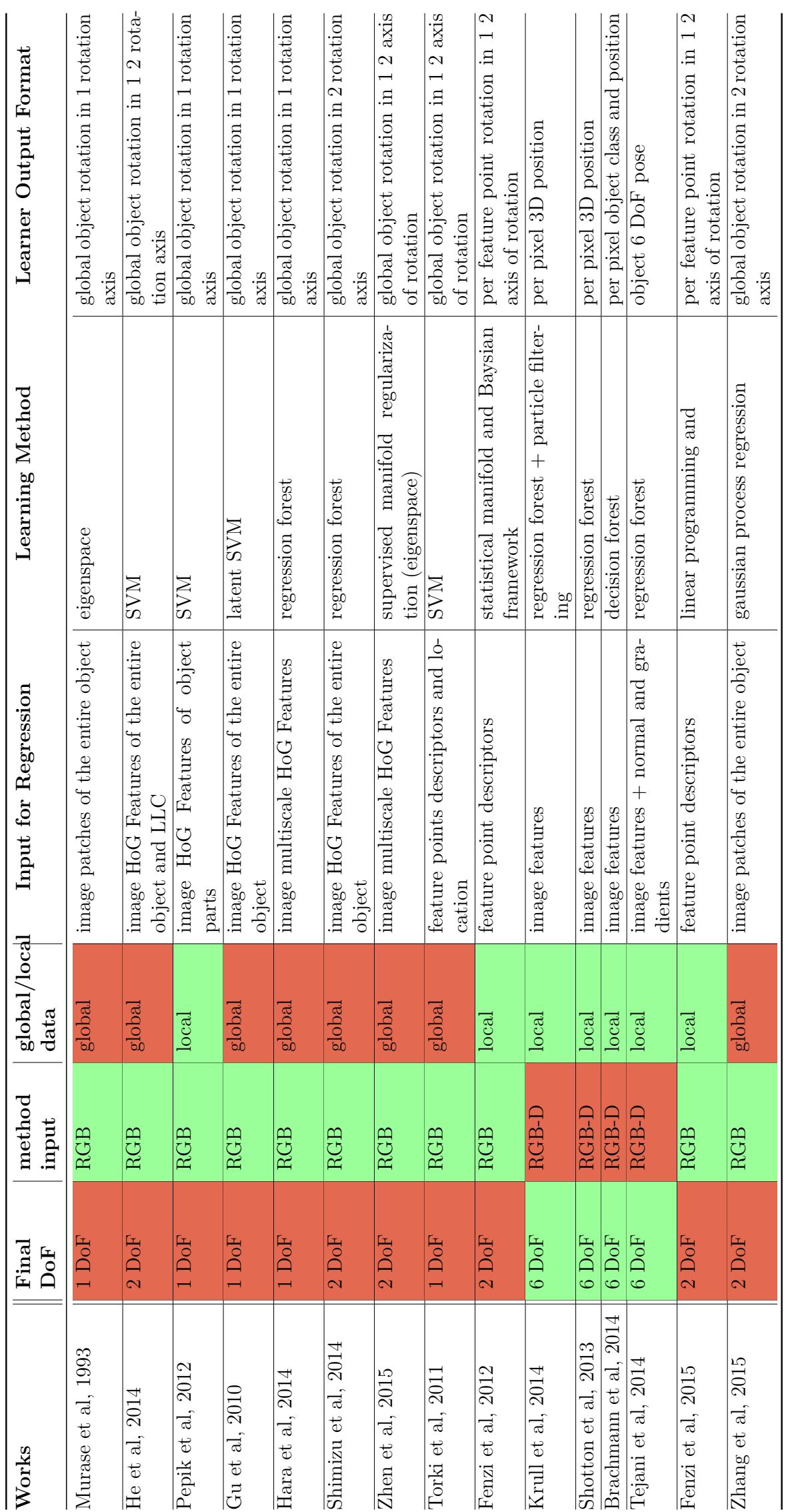

Source:Author. 
First, note that none of the works, which are based on RGB information, explicitly returns a full 6 DoF pose. This is because they lack in two key information in order to fully estimate the object poses; depth and third (camera) rotation. Although several works claims that this third (camera) rotation can be achieved simply by extending their methods, this is not true because of similarities and ambiguities in object appearance, which appear when full rotation is considered. Also, in some other works, such as ones based on HoG features, this extension is not simple, once its core information (HoG features) lacks in rotation invariance.

Depth information can be estimated once the full rotation is known. Thus showing the need of the third (camera) rotation, in order to obtain the full pose. On the other hand, if the depth information is known this third camera rotation can be estimated. This is explored in works that uses RGB-D information. Therefore, showing that full rotation estimation, and so, the full pose $(6 \mathrm{DoF})$ estimation, is not easily achieved only by RGB information unless explicitly modeled in the problem.

Additionally, none of the works based on RGB information returns full local pose or rotation. Works that are based on RGB-D information can explicitly estimate the local pose; despite that, these local poses are not explored further. Local pose is a rich information that enable novel approaches for pose estimation for several classes of objects, as presented in chapter 1 and will be explored along this work.

In summary, we can observe from this section that:

- Methods based on machine learning and local information, can avoid complex problem solving which appear in solve-PnP problems;

- They also enable local pose estimation in some level (1 or 2 DoF, for RGB based works; and full 6 DoF, for RGB-D based works);

- None of RGB based works presented in this section return full 6 DoF object pose, local pose or local rotation;

- None of RGB-D based works, although can estimate the local poses, explore this information in different cases.

\subsection{Deformable Object Pose Estimation}

In this section we show works that have focus on pose estimation of a different class of object, the deformable object. As presented in section 2.1.2, deformable objects differ from rigid object and are specially hard for $6 \mathrm{DoF}$ (degrees of freedom) pose estimation, once they cannot be represented by a single transformation, and also can have different constraints or assumptions. 
Pilet, Lepetit e Fua (2008) present a surface detection approach for augmented reality based on RGB information. In this work, image features points are used for matching (LEPETIT; LAGGER; FUA, 2005) a non-deformed image to the captured deformed condition. These image features are then molded into triangular meshes and the poses are estimated with an energy minimization approach. Once, image features of (LEPETIT; LAGGER; FUA, 2005) are used in this work, this work assumes the constraint of locally planar and rigid features points.

This locally rigid constraint is also found in works such as (PRITCHARD; HEIDRICH, 2003; LI; SUMNER; PAULY, 2008). Pritchard e Heidrich (2003) explores the local rigid constraint by finding feature points across the observed deformed cloth, and then, making the registration of the object. Li, Sumner e Pauly (2008), on the other hand, does not fully assume the locally rigid constraints, however, its registration method is based on maximization of energy with two functional. One, related to the surface smoothness of the alignment of the two observations, and another, that penalized the deviation of each transformation from a pure rigid motion. This second functional term is likely to preserve locally rigid parts of the observation, thus, exploring locally rigid parts of the deformation.

This locally rigid assumption is related to the shape of the object, as in (LI; SUMNER; PAULY, 2008), but also related to the appearance of the object texture, as in (PRITCHARD; HEIDRICH, 2003; PILET; LEPETIT; FUA, 2008). Thus, making possible the feature matching based on appearance using feature points.

Works of shapes-from-texture (MALIK; ROSENHOLTZ, 1997; GALASSO; LASENBY, 2007; GARDING, 1992; LOBAY; FORSYTH, 2006) also exploit the locally rigid assumption of the appearance in order to estimate the object shape. In those works, the deformed surface of some object can be estimated by the appearance change and repeat of known patterns places across the object surface.

Other type of constraint, that several works assume for deformable object pose estimation, are that object are piece-wise rigid or articulated(CHANG; ZWICKER, 2011; WESIERSKI; HORAIN, 2013; MICHEL et al., 2015; PAUWELS; RUBIO; ROS, 2014; PEKELNY; GOTSMAN, 2008). In this constraint, the object is assumed to have rigid parts, that can be matched across different views.

Chang e Zwicker (2011) and Pekelny e Gotsman (2008) find the non-rigid transformations of articulated objects by finding pieces that are rigid, and then approximating the transformations of the non-rigid parts. Pauwels, Rubio e Ros (2014) and Michel et al. (2015) consider that the piece-wise parts of the object are known a priori, in order to estimate the object poses, which is also a common prior knowledge assumed in several other works.

This constraint also diminish the number of parameters to be estimated, differently 
from other works, once large parts of the object (the rigid parts) can be estimated by a single rigid transformation matrix, related to each other. However, it is also possible to observe that this constraint contains the locally rigid assumption, once each rigid piece also contains locally rigid parts.

Another type of constraint that can be assumed, in order to estimate deformable objects pose, is that the object deformations and poses are known a priori. Several works of human body pose estimation, such as (ALLEN; CURLESS; POPOVIć, 2002; ALLEN; CURLESS; POPOVIć, 2003; ANGUELOV et al., 2005), consider this assumption. In those cases, space of the possible human poses are known, as well as, how skin can deform in those conditions.

Works such as (RENDL et al., 2014) also apply this assumption of deformable pose estimation. Rendl et al. (2014) use piezoeletric sensors spread over a transparent sheet of plastic, in order to estimate its deformation in combination with k-nearest neighbour algorithm.

This assumption has the advantage of being able to estimate how are the deformations of the object, even of occluded of parts. However, in order to achieve this assumption, all the poses need to be mapped, which can be hard for some cases of deformation. Additionally, if the observed object takes some non-trained pose, it can crash the estimation.

Finally, spatio-temporal constraint can also be explored in order to track the object pose. In this case, is assumed that the object geometry does not have large changes across consecutive temporal observations. Works such as (JORDT; KOCH, 2013; PAULUS et al., 2015) explores this assumption.

However, this assumption also means that consecutive time samples of the object needs to be collected. This makes those methods a tracking problem (FAUGERAS, 1993), thus, being hard to estimate the object poses in any time step, and depending of initialization pose. Since, in this work we focus on direct pose estimation, those constraints can not be used. Moreover, spatio-temporal assumption can be achieved if other assumptions are also true.

In table 3 we present a quick overview of the works presented here and their assumption for deformable object pose estimation. In table 4, we also present the correlation between the assumptions that where presented in this section. Each row presents a assumption over the deformable object, and which other assumptions this row's assumption also can satisfy (each column).

From table 4, it is possible to observe that locally rigid is one of the assumptions that can satisfy other assumptions. spatio-temporal assumption also can satisfy several other assumptions, however, depends on temporal information. 
Table 3 - Comparison of works for deformable object pose estimation and its assumptions.

\begin{tabular}{lll}
\hline works & input type & deformation assumption \\
\hline Pilet et al., 2008 & RGB & locally rigid \\
Pritchard et al., 2003 & Stereo RGB & locally rigid \\
Li et al., 2008 & depth map & locally rigid (not fully) \\
Malik et al., 1997 & RGB & locally rigid \\
Galasso et al., 2007 & RGB \\
Garding et al., 1992 & RGB & locally rigid \\
Lobay et al., 2006 & RGB & locally rigid \\
Chang et al., 2011 & depth map & piece-wise rigid \\
Wesierski et al., 2013 & RGB (pose in 2D) & piece-wise rigid \\
Michel et al., 2015 & RGB-D & piece-wise rigid \\
Pauwels et al., 2014 & Stereo RGB & piece-wise rigid \\
Pekelny et al., 2008 & depth map & piece-wise rigid \\
Allen et al., 2002 & depth map & known deformation \\
Allen et al., 2003 & multiple range observations & known deformation \\
Angelov et al., 2005 & depth map & known deformation \\
Rendl et al., 2014 & piezoeletric sensors & known deformation \\
Jordt et al., 2013 & RGB-D & spatio-temporal \\
Paulus et al., 2015 & RGB & spatio-temporal \\
\hline
\end{tabular}

Source:Author.

Table 4 - Correlation of object deformation assumptions.

\begin{tabular}{|c|c|c|c|c|}
\hline & locally rigid & $\begin{array}{l}\text { piece-wise } \\
\text { rigid }\end{array}$ & $\begin{array}{l}\text { known defor- } \\
\text { mation }\end{array}$ & $\begin{array}{l}\text { spatio- } \\
\text { temporal }\end{array}$ \\
\hline \multirow{2}{*}{$\begin{array}{l}\text { locally rigid } \\
\text { piece-wise } \\
\text { rigid }\end{array}$} & $\longrightarrow$ & $\mathrm{OK}$ & $\mathrm{OK}$ & OK \\
\hline & no & $\longrightarrow$ & $\mathrm{OK}$ & $\mathrm{OK}$ \\
\hline $\begin{array}{l}\text { known defor- } \\
\text { mation }\end{array}$ & $\begin{array}{l}\text { OK (if } \\
\text { trained) / no } \\
\text { (untrainned } \\
\text { case) }\end{array}$ & $\begin{array}{l}\text { OK (if } \\
\text { trained) / no } \\
\text { (untrainned } \\
\text { case) }\end{array}$ & $\longrightarrow$ & $\begin{array}{l}\text { OK (if } \\
\text { trained) / no } \\
\text { (untrainned } \\
\text { case) }\end{array}$ \\
\hline $\begin{array}{l}\text { spatio- } \\
\text { temporal }\end{array}$ & $\begin{array}{l}\text { OK (tracking } \\
\text { problem) }\end{array}$ & $\begin{array}{l}\text { OK (tracking } \\
\text { problem) }\end{array}$ & $\begin{array}{l}\text { OK (tracking } \\
\text { problem) }\end{array}$ & \\
\hline
\end{tabular}

Object deformation training also can satisfy the other constraints, if, the deformation cases of the object is known. Despite that, it is hard to know all the deformations cases depending on the object to be estimated. Making it restrict to some object deformations.

From this section we can now observe that:

- Spatio-temporal constraint is used in several works for deformation estimation, but 
are a set of tracking problems;

- Knowing and training the object deformation also can be used in pose estimation, but can be hard to extensively sample the deformation possibilities;

- Locally rigid constraint appear in several conditions of deformation and does not imposes huge constraints.

\subsection{Discussions}

In sections 3.1, 3.2, 3.4 and 3.3 several works about object pose estimation where presented, showing that solutions of pose estimation can widely variate according to object type and capturing conditions. However, we also can observe that several solutions and methods in literature simplify the known object information to simple points in space.

On the other hand, works such as (CHOI; CHRISTENSEN, 2012; MARCON et al., 2012; MURASE; NAYAR, 1993; PEPIK et al., 2012; TORKI; ELGAMMAL, 2011; BRACHMANN et al., 2014; SHOTTON et al., 2013) presents that more information can be gathered from object in order to be used directly in the object pose estimation (PEPIK et al., 2012), or to polish its result (MARCON et al., 2012). Also, local information of the object can be used in order to even estimate the poses of deformable objects (RENDL et al., 2014). Here in this section, we will discuss about the presented works and our solutions for object pose estimation; differences and similarities between our solution and the works in literature, as well as our characteristics.

Initially, it is possible to observe from section 3.4 that several deformable objects share the characteristics of being locally rigid. Thus, being able to track those rigid parts can lead us to estimate the poses of deformable objects; even without the need of temporal information nor extensively sampling the possible deformations of the object. Therefore, we will focus on the locally rigid assumption of the object for pose estimation in AR in this work. Moreover, we take another simplification of this assumption, considering that the object is locally rigid and planar. This assumption can gives us several advantages across this work, which will be presented in the next chapters; and still can be used in several object classes (LEPETIT; LAGGER; FUA, 2005).

Also, more spatial knowledge from the surface, such as normal or direction (MARCON et al., 2012; CHOI; CHRISTENSEN, 2012; BRACHMANN et al., 2014; LI; SUMNER; PAULY, 2008), can give powerful information for pose estimation for rigid (MARCON et al., 2012; CHOI; CHRISTENSEN, 2012; BRACHMANN et al., 2014) or deformable objects (LI; SUMNER; PAULY, 2008). Despite that, none of the works presented here explicitly explore the full local 6 degrees-of-freedom (DoF) pose. Thus, in this work we explores local poses as key aspect for global object pose estimation. 
From the locally rigid assumption, we also can assume that local texture appearance of the object do not suffer major changes across deformation; which in turn, also means that feature points over the object surface do not change its appearance by the deformation itself. Making possible the usage of conventional feature points extraction and matching methods (chapter 2.6) in our approach.

However, this also leads to the fact that the same features point should be observed in several view conditions, in other words, different affine transformations. As presented in section 2.6.2, conventional feature points are not affine invariant. Therefore, we also extend the ASIFT (MOREL; YU, 2009) work for AR systems, in order to extract robust feature points across different views by our method presented in chapter 6 .

Also, methods to be applied for pose estimation vary widely with the capturing setup, specially if the depth information can be captured using RGB-D cameras. Both RGB and RGB-D setups are based on devices of image capturing. Several works achieve real-time pose estimation and tracking based only on 2D color images information with similar setup, as shown at section 3.2.

On the other hand, depth cameras has been widely available as input source, even as commercial solutions. These cameras can be based on different technologies, but in all the cases it can return spatial 3D information as depth maps. These depth information can enable more simple and high-precision pose estimation method. However, it also means that another special hardware is needed other than conventional RGB cameras.

Either setups are common in different state-of-art solutions and it is hard to determinate which should be better for our local pose estimation approach. Due to this problem both conditions are studied is this work. In the next subsections 3.5.1 and 3.5.2, we present discussion over each capture conditions, about our approaches and the differences with literature works.

\subsubsection{Object pose with RGB-D}

First, we present our solution for pose estimation based on RGB-D capturing setup in this work. This is due to the simplicity that the spatial information of depth can bring for pose estimation. However, also due to this simplicity, several works simplify the spatial information to single dots in space as presented in sections 3.1 and 3.4.

Our approach differ from those works once we focus on extracting explicit local pose of each found feature point (which we consider to be locally rigid and planar). (CHOI; CHRISTENSEN, 2012) is one of the works that is most similar to our solution, where the observed normal, position and second vector direction are aligned with the pre-storage ones. This process is similar to our method of local pose estimation, chapter 7. Though, local explicit pose is not extracted in (CHOI; CHRISTENSEN, 2012), nor its usage in 
deformable objects.

Our solution also differ from (MARCON et al., 2012), where dense normal of the object is estimated by PCA methods. Here, the calculation of the surface normal can be largely simplified to a cross product between two vectors that lay at the object surface, once we assume that the object is locally planar.

Finally, our work shares similarities with (RENDL et al., 2014) for deformable objects. In (RENDL et al., 2014), the deformable object poses are estimated by training the sensor outputs to possible poses known as priori. Although we do not need to use training for pose estimation, the original poses of the deformable object in (RENDL et al., 2014) are represented as locally rigid poses of the sheet captured by fiducial markers. Thus showing that knowing the local poses of the object could lead to similar results.

\subsubsection{Object poses with RGB}

Despite the lack of full spatial information, methods based only on RGB information still can enable good results for pose estimation, such shown at section 3.2 and 3.3. This is achieved by knowing information of the object pose to be estimated and finding the correlation between the observed information.

Also, papers presented in section 3.3 show that local knowledge of near surrounding regions of some points, can give rich information of the object pose itself. Moreover, machine learning based approaches can replace the complex high-order non-linear equations solving problem (KUKELOVA; BUJNAK; PAJDLA, 2008) presented in subsection 3.2.1; replacing it with a fast and robust approach with dramatically lower calculation demands (CRIMINISI; SHOTTON, 2013).

Despite that, none of the works presented in section 3.3 based on RGB information can fully estimate the object pose, nor the local poses. Details will be presented in chapter 8 , but one strong issue is the appearance similarities that appear between views, when considering full pose. In this work, we address this problem, using rotation and scale invariant feature points local information in order to estimate the local rotation of each feature point; and than, obtain the full 6 DoF pose of the object.

Our method for full pose estimation is similar to (SHOTTON et al., 2013) and (BRACHMANN et al., 2014), where random forest regression method is combined with RANSAC method (FISCHLER; BOLLES, 1981), in order to filter the trees results. Although, both works are based on RGB-D information.

Our work also is similar to (FENZI et al., 2013) solution, where rotation and scale invariant feature points are used to train the pose regression; and (PEPIK et al., 2012), based on piece-wise HoG features. Those approaches makes regression results local. But, again, both do not enable full pose estimation nor full local rotation. 


\subsection{Chapter Conclusion}

In this chapter we have presented related work to our local pose estimation idea. From this chapter we can observe that:

- Several works simplify the feature points or known object information to single points in space, not exploring the local information;

- Some RGB-D works explore local information for rigid and deformable objects;

- Some RGB works explores the local information and machine learning of rigid and deformable objects; however none can obtain full object pose;

- None works presented here explores the local pose for pose estimation of different types of objects.

Those observations gives foundation for our work for pose estimation, as well as our contributions, presented in chapter 7 and 8 . In the next chapter, 4 , we first present the assumptions taken in this work, based on the related work for pose estimation. 


\section{Assumptions}

Due to several assumptions that we consider across this work, initially, we will present these assumptions about our capture environment and object surface model, before presenting our methods itself. First, assumptions about the object surface model to be estimated will be presented at section 4.1.

Then, in section 4.2, we show assumptions related with the capturing environment. Section 4.3 will present how to achieve these assumptions in real world scenarios. Finally, in section 4.4 we briefly recapitulate all the assumptions, as well as, all the expected input data for our methods.

\subsection{Object Surface}

The first assumptions $A_{o 0}$ that we consider here, is that the object surface is textured. This means that the object has enough diffuse color changes across the surface to generate feature points (LOWE, 1999; BAY et al., 2008). Also, meaning that poses of objects with plain surface color can not be estimated by our methods.

A second assumption, $A_{o 1}$, is that the reflectance model of the object surface follows the Lambertian model (ANGEL, 2006). Meaning that the object surface is not specular nor transparent, but perfectly diffuse. $A_{o 0}$ and $A_{o 1}$ are conventional assumptions that different vision works assume to be true (chapter 3).

Now, our major assumption, $A_{o 2}$, on this work is that the object surfaces around feature points are locally planar and does not deform with changes across time, as show in figure 10. This assumption, also considered in (LEPETIT; LAGGER; FUA, 2005), leads to several characteristics that will be used across this work.

One characteristic generated by this assumption is that features points to be extracted are not generated by the surface geometry, but from the surface texture itself. With this, these feature points are more robustly found across perspective, once does not suddenly changes its description by camera position changes. Also, it is possible to consider that a large amount of the feature points orientations are generated only by the surface texture, also not changing across perspective changes. Moreover, once it is considered to not deform across time, meaning it is locally rigid, it makes possible the pose estimation of some deformable objects.

Another assumption $A_{o 3}$ that we consider is that the object surface texture $I_{\text {tex }}$ and the function $f_{o b j}\left(T_{t e x}\right)=T_{o b j}$, that maps surface texture space transformation to the object space transformation (figure 11 illustrates this function), are known. This assumption is 
Figure 10 - Locally planar surface assumption.

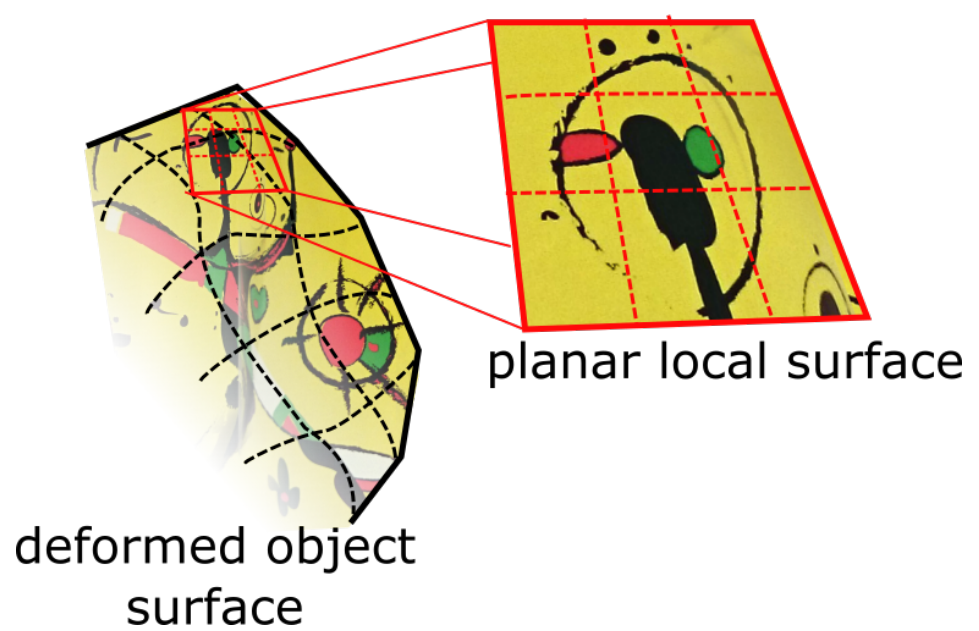

Source: Author.

related with the object geometry and texture mapping, and they are used in order to find the local and global pose of the object. Once the local pose of the feature is estimated, this local pose is transformed by this function $f_{o b j}\left(T_{t e x}\right)$, giving the global pose, as shown at figure 11.

Figure 11 - texture mapping function, $f_{o b j}\left(T_{t e x}\right)$, illustration. Using this function and its inverse, it is possible to convert the local planar transformation to the original object space and vice-versa.

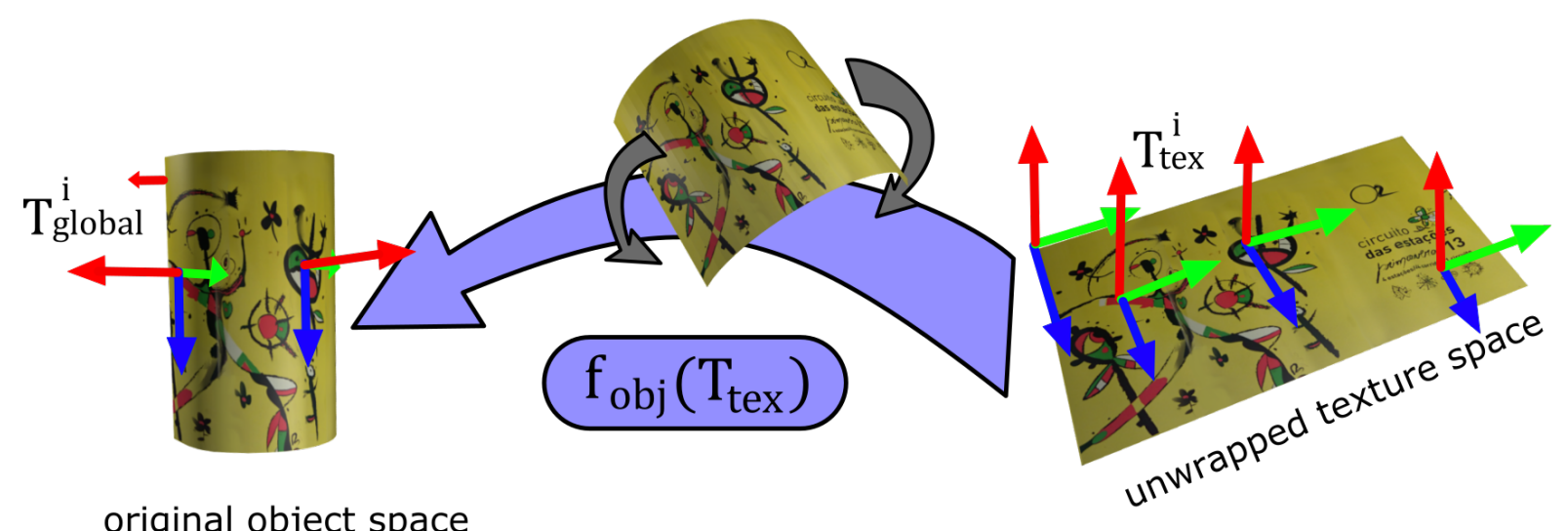

Source: Author.

So far, the base assumptions that we consider about the object surface are:

- $A_{o 0}$ : textured object surface;

- $A_{o 1}$ : Lambertian surface reflection;

- $A_{o 2}$ : Locally planar and rigid feature points;

- $A_{o 3}$ : Known $I_{\text {tex }}$ and $f_{o b j}\left(T_{t e x}\right)=T_{o b j}$. 


\subsection{Capture Environment}

Some assumptions about the capture environment will vary according the capture environment setup. In this work, we consider two different capturing setups.

- with conventional RGB camera;

- with RGB-D camera, which can capture depth map information of the scene with the RGB image.

One assumption, $A_{c 0}$, taken in both setups, is that a conventional camera with known projection matrix, $\mathbf{K}_{\text {color }}$, is used in order to capture the RGB image $I_{\text {color }}$. Also, one minor assumption that we consider, is that the camera image does not has radial distortions, as a pin-hole camera, $A_{c 1}$. This two assumptions lead to the projection matrix with the format

$$
\mathbf{K}_{\text {color }}=\left[\begin{array}{ccc}
f & 0 & O_{x} \\
0 & f & O_{y} \\
0 & 0 & 1
\end{array}\right],
$$

where $f$ is the focal point, $O_{x}$, horizontal correction and $O_{y}$, the vertical correction value. Note that conventional projection matrices can have two different focal points values for $x$ and $y$. However, having different values, leads to scale distortion of the observed image, therefore, in this work, we consider that the focal point values are approximately equal.

In the second setup, it is assumed that the depth image $I_{\text {depth }}$ is aligned and rectified to the color image $I_{\text {color }}, A_{c 2}$. Meaning that, the same pixel position at color image space points towards the same position at the depth image space. This assumption is also adopted by works that base on RGB-D cameras.

So far, three base assumptions are made about the capturing setup:

- $A_{c 0}$ : Known $I_{\text {color }}$ and $K_{\text {color }}$;

- $A_{c 1}$ : No image distortion;

- $A_{c 2}$ : Known and rectificated $I_{\text {depth }}$;

\subsection{Real World Scenarios}

In real world conditions, some of the assumptions listed above are not achieved directly. Therefore, in order to reach these assumptions, calibrations or selections of the object and feature points can be applied. Initially, the assumption $A_{o 0}$ and $A_{o 1}$ can be reached by limiting the object materials to be estimated. This restriction is found in several 
works of object pose estimation (LEPETIT; LAGGER; FUA, 2005; TORKI; ELGAMMAL, 2011; LEPETIT; MORENO-NOGUER; FUA, 2009).

The locally planar and rigid assumption $A_{o 2}$ is adopted also by works as (LEPETIT; LAGGER; FUA, 2005; PRITCHARD; HEIDRICH, 2003; MOREL; YU, 2009). This assumption can be reached by the process of feature points extraction. Once the feature points are assumed to be locally planar, selecting features that does not suddenly change and have similar spacial position can filter points that do not have these characteristics.

Then, in order to achieve assumption $A_{o 3}$ the geometry and texture mapping of the object needs to be known. In several cases, other than planar, spherical or cylindrical objects, this geometry and texture acquisition is not direct. However, methods of geometry reconstruction presented by (IZADI et al., 2011; MATUSIK et al., 2000; TUNWATTANAPONG et al., 2013) can be applied in order to capture this geometry and texture mapping. These methods returns the geometry of the object, as well as the object texture and the texture map around the geometry. Also, in case that the texture is deformed in the mapping function, methods of geometry unfolding that preserves the geometry deformation as (MITANI; SUZUKI, 2004; MASSARWI; GOTSMAN; ELBER, 2007) also can be applied.

Assumption $A_{c 0}$ can be achieved by camera calibration methods as presented at (ZHANG, 2000; HEIKKILA; SILVEN, 1997; STURM; MAYBANK, 1999). These methods use known patter images taken with the color camera in different positions, and return the full projection matrix $\mathbf{K}_{\text {color }}$.

If the camera has distortions in the projection matrix, it can break the assumption $A_{c 1}$. However, this can be easily corrected by applying image distortion correct algorithms (ZHANG, 2000), forcing the image to have a projection matrix as 4.1 .

In case of assumption $A_{c 2}$, several hardware setups using time-of-flight or lightfield technologies can achieve this assumption directly. However, in case of structured light technologies or in setups that uses a separated color image sensor, other than the depth camera sensor, the images $I_{\text {color }}$ and $I_{\text {depth }}$ will not be aligned, breaking $A_{c 2}$. In order to achieve this assumption, image rectification methods can be applied (TRUCCO; VERRI, 1998).

\subsection{Chapter Conclusions}

Here, in this chapter, we have presented our base assumptions about the object model, as well as, the methods to reach these assumptions. Our full list of base assumptions are: 
Table 5 - Full list of assumptions of this work.

\begin{tabular}{l||l}
\hline$A_{o 0}$ & textured object surface; \\
\hline$A_{o 1}$ & Lambertian surface reflection; \\
\hline$A_{o 2}$ & Locally planar and rigid feature points; \\
\hline$A_{o 3}$ & Known $I_{\text {tex }}$ and $f_{\text {global }}\left(T_{\text {tex }}\right)=T_{\text {local }} ;$ \\
\hline$A_{c 0}$ & Known $I_{c o l o r}$ and $\mathbf{K}_{\text {color }} ;$ \\
\hline$A_{c 1}$ & No image distortion; \\
\hline$A_{c 2}$ & Known and rectified $I_{\text {depth }}$. \\
\hline
\end{tabular}

Source: Author.

These assumptions delimit our possibility space, in order to make the different pose estimations possible. Further explanations of how they are used will be explained in the following chapters. Also, they define the inputs that our approach needs from the capturing system. These input information are:

Table 6 - List of expected input information for our approach.

\begin{tabular}{l||l}
\hline$I_{t e x}$ & object surface texture image; \\
\hline$f_{\text {obj }}\left(T_{t e x}\right)$ & $\begin{array}{l}\text { mapping function of the texture image space to the global object } \\
\text { space; }\end{array}$ \\
\hline $\mathbf{K}_{\text {color }}$ & projection matrix of the color camera; \\
\hline$I_{\text {color }}$ & RGB image frame; \\
\hline$I_{\text {depth }}$ & $\begin{array}{l}\text { depth map image, rectified with } I_{\text {color }} ; \text { in case of RGB-D camera } \\
\text { setup. }\end{array}$ \\
\hline
\end{tabular}

Source: Author.

In the next chapter 5 we will present the overview of our local pose based approach, showing how our approaches work and the information flow of this work. 


\section{Local Pose Based Approach Overview}

In this chapter we present the basic concept and overview of our pose estimation approaches based on local poses. This idea and approaches themselves are part of the core contributions of this work, once they provide new ways for object pose estimation methods.

Conventional methods use feature points information directly for global pose estimation, without gathering more information from each point and its surrounding, which can give important clues of the object pose. These information are explored in this work. In section 5.1 we present the general concept; and in section 5.2, we present the information flow of the entire process.

\subsection{Base Concept}

The basic concept of this work is to locally found the pose of feature points spread across the object surface. Each local pose gives us clues of how the object is transformed. Figure 12 illustrates this local poses and how they can give clues of the pose of different object classes.

In case of planar objects, figure 12a, each found local pose are equal to the global planar object pose, since it is a rigid object. Furthermore, each local poses also leads to the object global pose in case of rigid 3D objects; after they are rectified by $f_{\text {obj }}\left(T_{\text {tex }}\right)$. Such presented at assumption $A_{o 3}$ at chapter 4.1, and illustrated in figure $12 \mathrm{~b}$.

Even deformable objects poses can be estimated once local pose are known, as illustrated in figure 12c. This deformable pose estimation idea is specially explored in (RENDL et al., 2014), which explores the local deformation to estimate the object poses.

Thus, it is possible to observe that local pose are directly related with the global pose of different classes of object. Characteristics, which are explored in this work in order to achieve the object pose estimation for AR registration. Moreover, unlike other works, we explicitly extract the local 6 DoF pose of points spread over the object surface, bringing richer information at the global pose estimation process.

In order to achieve the local poses, we use feature point extraction and matching methods to find interest points spread over the object surface. After finding these interest points, we use the information of the surrounding surface to extract its pose. This local pose estimation will vary depending of the information that we have in hands.

In common capturing setups, only color projections of the texture around feature 
Figure 12 - Local poses relationship to the object global pose.

(a) Planar case. First image: observation; second: found feature points; third: found local poses, represented by the RGB coordinate system over the corresponding feature point; forth: global object pose. Note that the global pose is the same as the local poses.
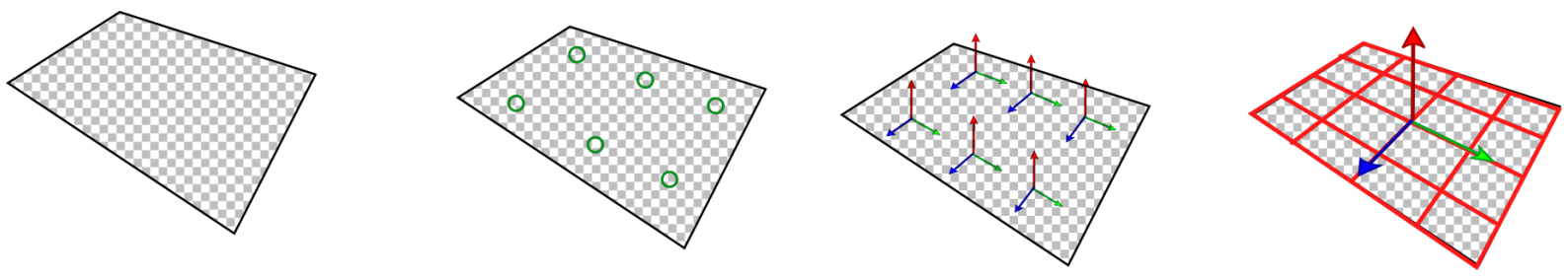

(b) Rigid 3D object case. First image: observation; second: found feature points; third: found local poses of the object surface; forth: rectified local poses; fifth: global object pose. Note that the global pose is the same as the rectified local poses.
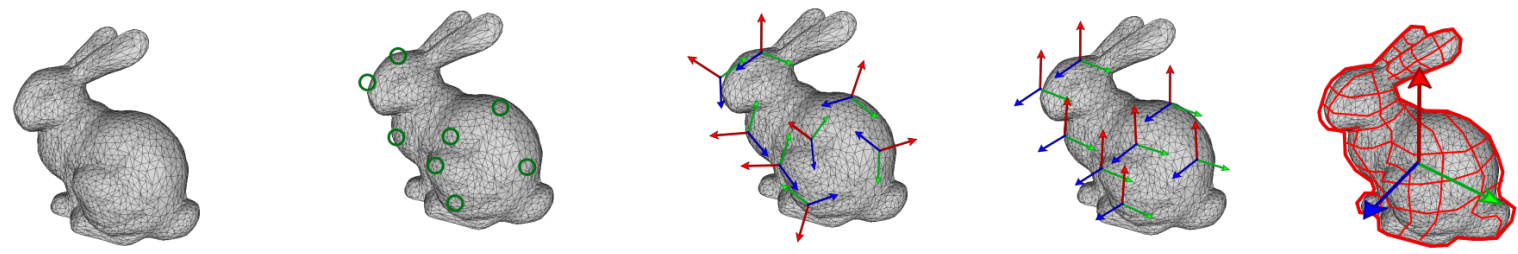

(c) Deformable object case. First image: observation; second: found feature points; third: found local poses; forth: global object pose. Note that the global deformation can be calculated by the local poses.
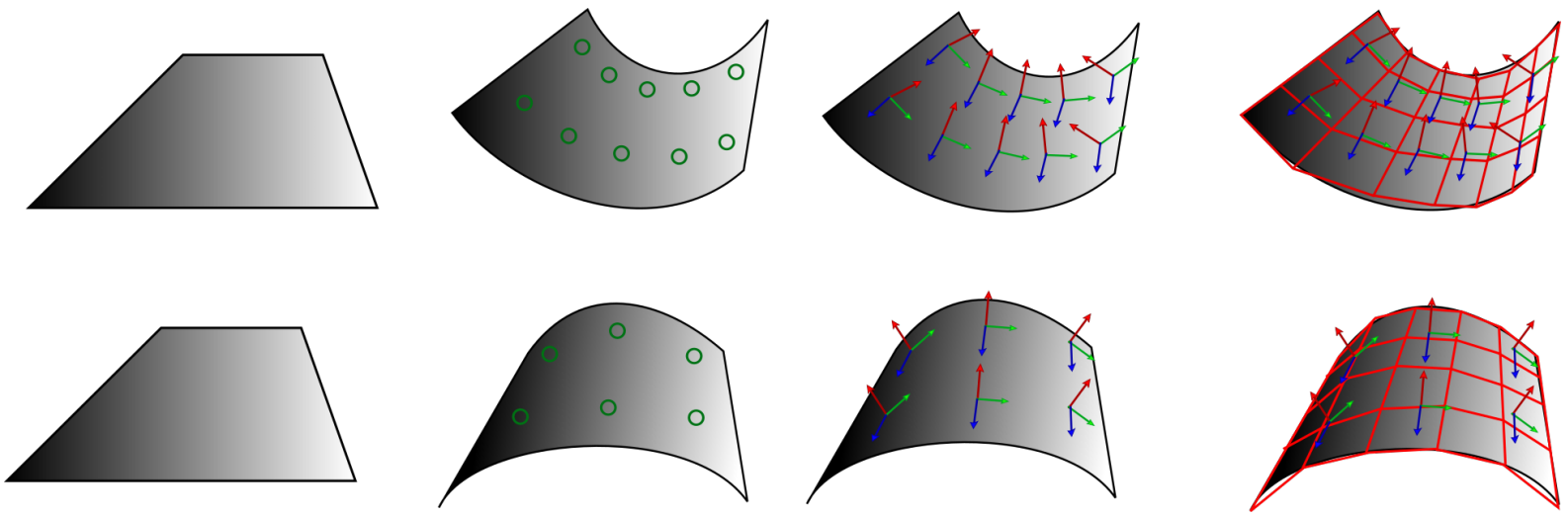

Source: Author.

points are available. In those cases, we estimate the local pose by the image deformation created by the projection, as shown in figure 13 and explored in chapter 8. On the other hand, RGB-D cameras can give spatial information of the environment as presented at 4.2. In these case we use the depth information with the texture orientation to estimate the local feature point pose, as presented in figure 14 and explored in chapter 7. 
Figure 13 - Appearance changes of the image patch with same location and same orientation.

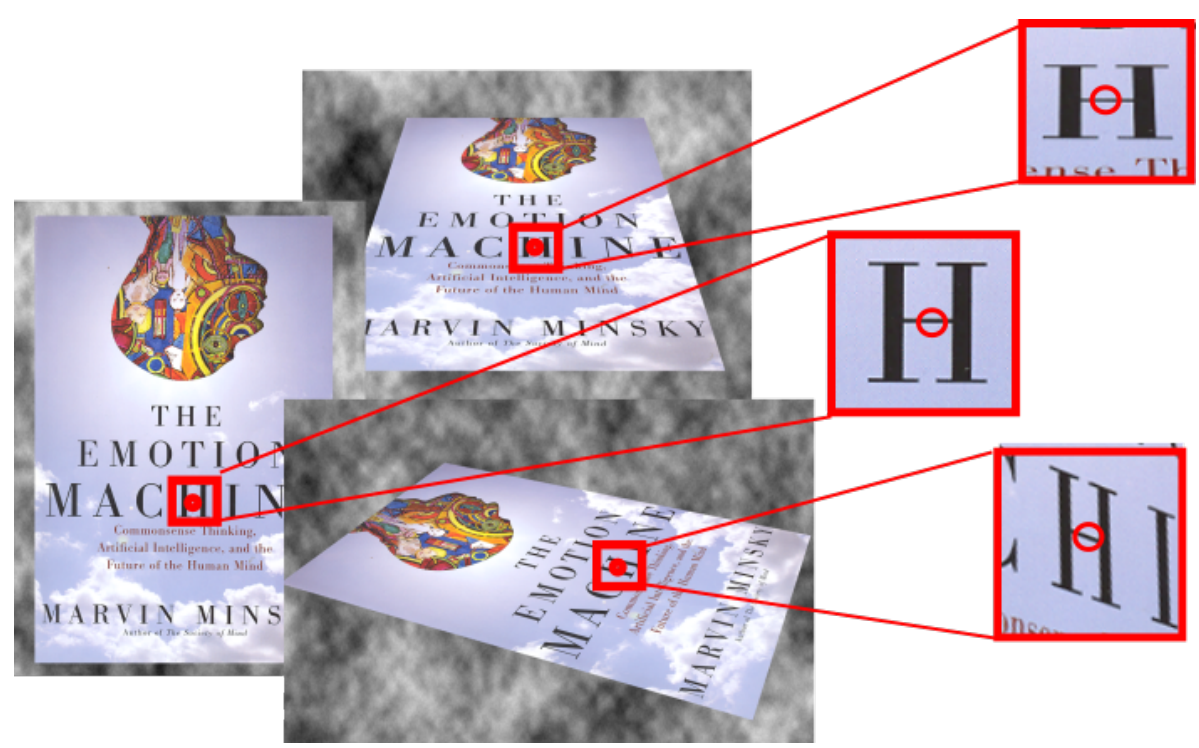

Source: Author.

Figure 14 - Local RGB-D information to object pose.

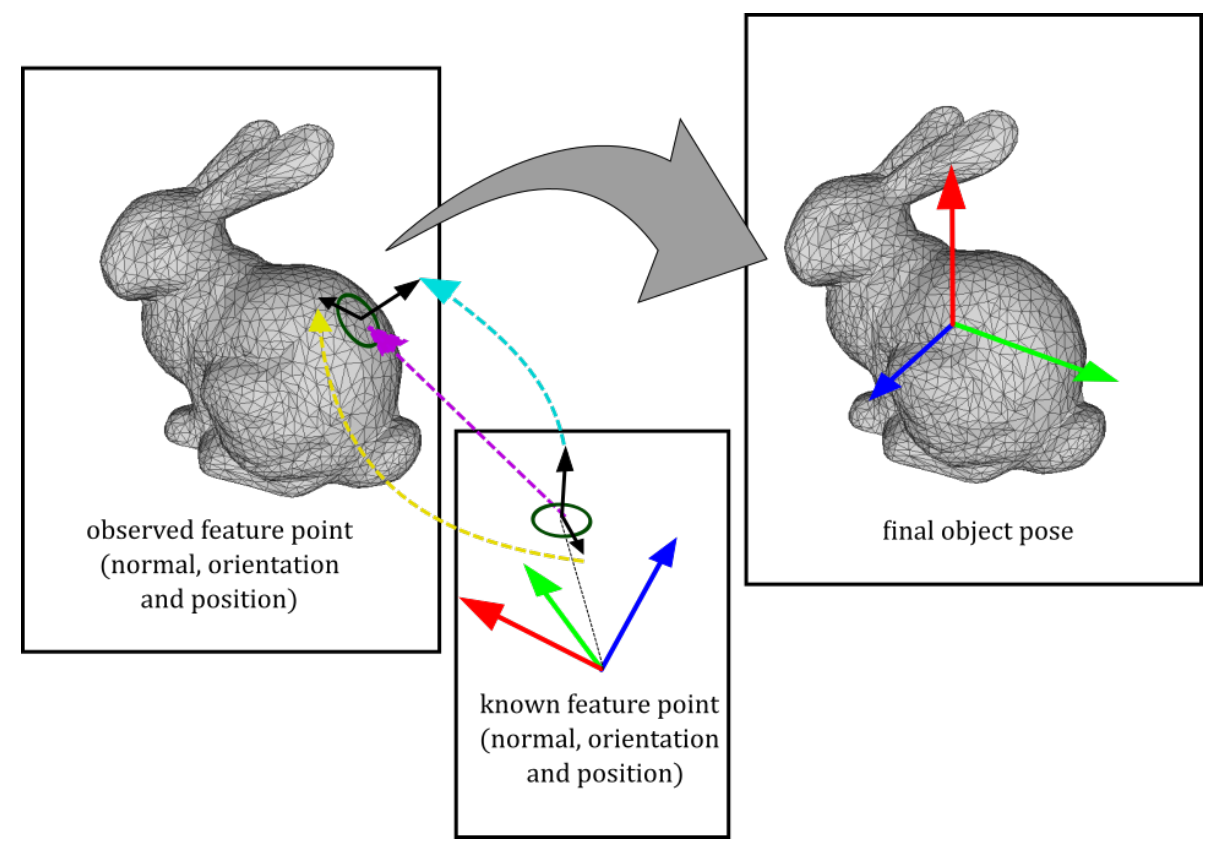

Source: Author.

\subsection{Information Flow}

The full process of pose estimation based on local poses can be represented by 2 major parts. The pre-processing part and real-time process part. In the pre-processing part we extract the information of the object in order to be used at the real-time processing. Figure 15 illustrates these parts in the complete information flow of this work. 
Figure 15 - Information flow. $F_{o b j}$ is the extracted feature points; $T_{\text {local }}$, the local transformation; $R_{\text {local }}$, the local rotation; and $T_{\text {final }}$ is the final object pose. The robust feature point extraction part will be presented in chapter 6 ; RGB process, in chapter 8; and RGB-D process, in chapter 7.

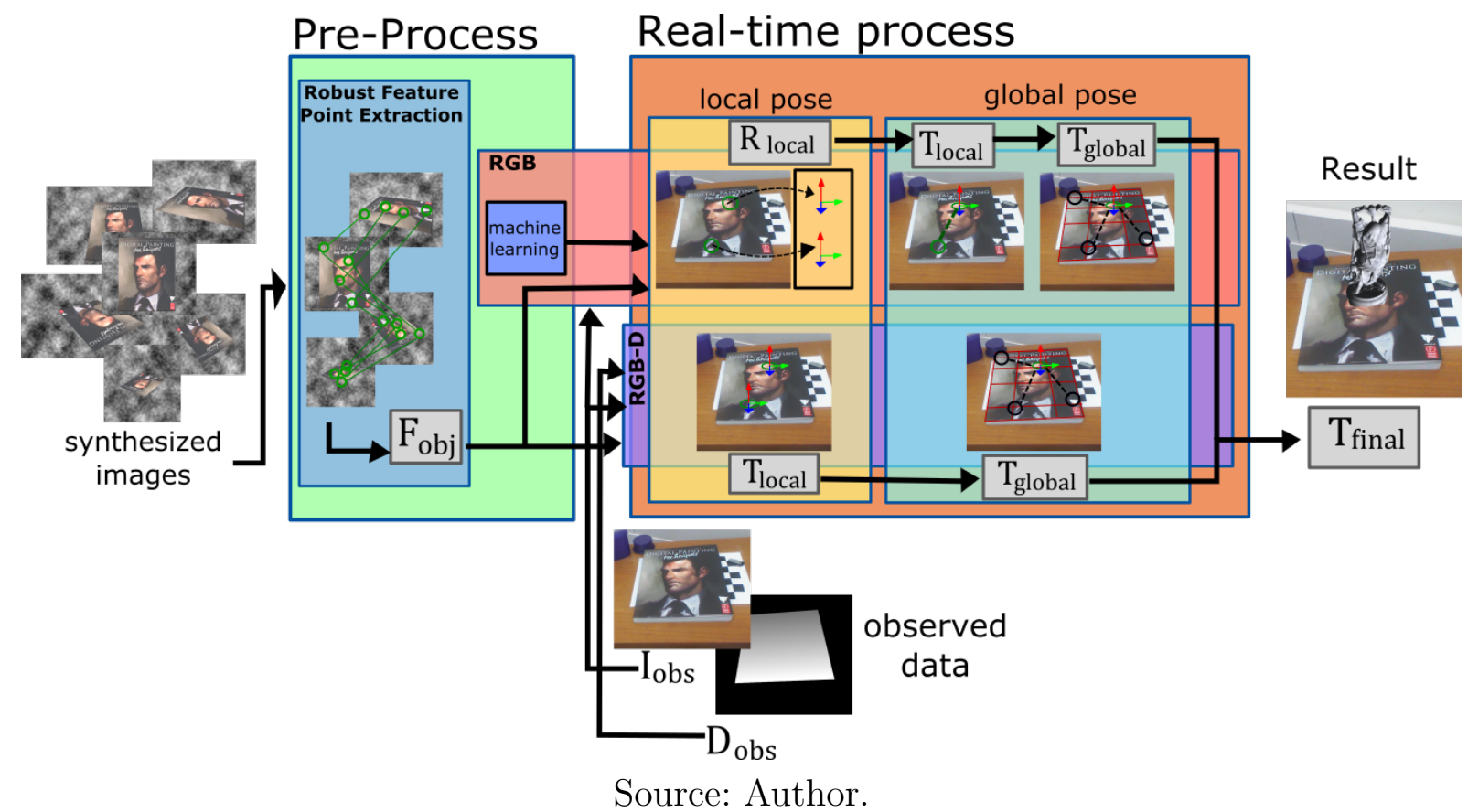

Inside the pre-processing part, we have two groups of processes, the robust feature point extraction and the local information extraction. From the robust feature point extraction, we find the interest points, $F_{o b j}$, spread over the object surface, then, this information is used in order to extract the local information and combined at $F_{o b j}$.

At the real-time processing part, we first use the feature points information to extract and match feature points in the new frame $I_{\text {obs }}$. Then, based on the local information within $F_{o b j}$, we calculate the local transformations $\mathbf{T}_{\text {local }}$. Finally, $\mathbf{T}_{\text {local }}$ are processed in order to find the final object pose $\mathbf{T}_{o b j}$. The local information extraction, in pre-process part; and local pose estimation process, at real-time processing part, will differ according the capturing setup, but both return similar information.

\subsection{Chapter Conclusions}

Here, we presented an overview of our pose estimation approaches based on local poses, as well as, the information flow of the entire work. The local pose estimation pre-process and estimation based on RGB-D information will be presented in 7 , and, based on conventional RGB information, in chapter 8. In the next chapter, we will explain about our methods of robust feature extraction, at the pre-processing part, that will return the feature points to be estimated, based on our assumptions and input information. 


\section{Robust Feature Points Extraction}

Figure 16 - Information flow of our robust feature points extraction.
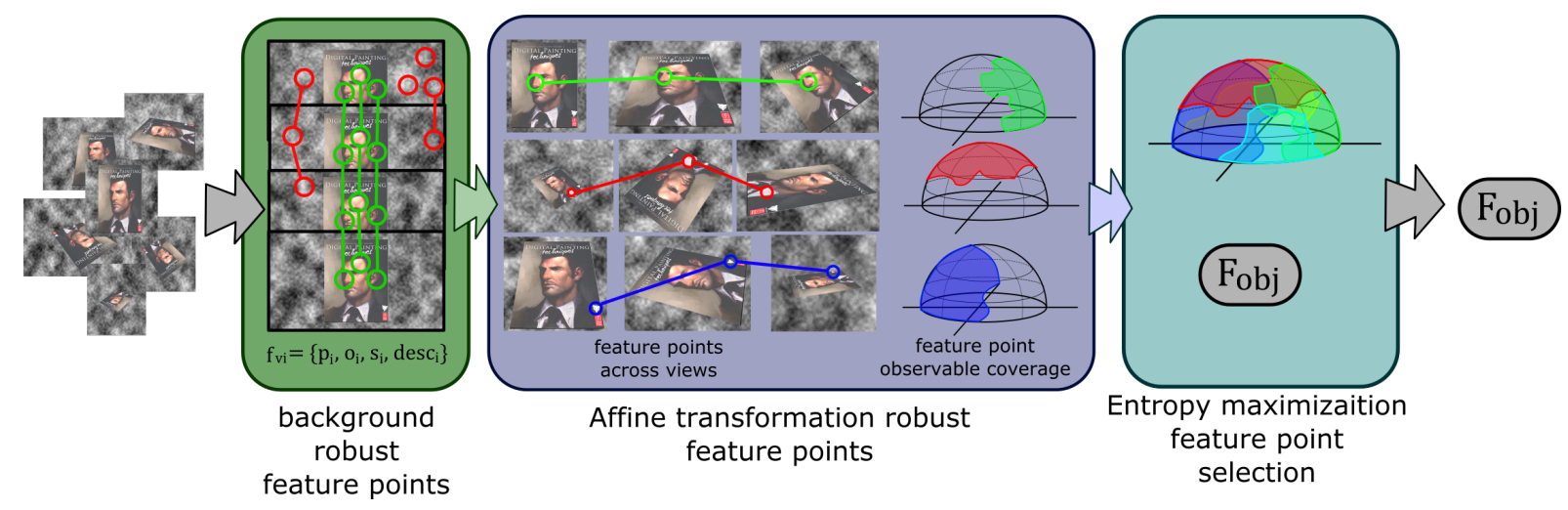

Source: Author.

In order to execute the pose estimation, first, we need to extract robust feature points of the object surface. In this chapter, we describe our robust feature points extraction process. The goal here is to extract feature points that are robust through camera transformations and background changes. The information flow of this extraction is illustrated in figure 16.

Here, different classes of feature point detectors and descriptors that are invariant in scale and orientation can be used, such as SIFT (LOWE, 1999) or SURF (BAY et al., 2008) feature points. In this work we have chosen SURF feature point as detector and descriptor, once it gives good balance between process speed and description match, also it present some characteristics that will be used in our method. However, other variants as CSIFT (ABDEL-HAKIM; FARAG, 2006) also could be used.

SURF features points (BAY et al., 2008) are detected using Hessian-Laplace detectors. Bay et al. (2008), authors presents a approximation of this detector using Gaussian Haar wavelets. Based on these responses in the horizontal and vertical orientation of the image, the major orientation of the feature point is computed. With this, SURF feature returns blob like feature points with a major orientation gradient. This characteristic is important and used at the pose estimation process described in the following chapters.

In the following sections, we will describe processes applied over this SURF feature detector in order to turn it robust to background changes and affine transformations. 


\subsection{Locally Planar Assumption $A_{o 2}$ and feature Extraction}

Here, the main task is to extract the features points that are spread over the object surface. In case of 3D objects, this extraction can be harder than planar objects, once different features can appear or disappear according the object pose, due to self-oclusions.

However, once that assumption $A_{o 2}$ at table 5 (feature points are locally planar) is adopted, it is possible to consider that all the feature points that we need to extract from the object texture $I_{t e x}$ are also planar. So, it is possible extract them also from a planar rendering of the texture image, as illustrated in figure 11 at section 4.1 .

This assumption can simplify the feature extraction model, and also, allow that the feature point extraction of different object classes be the same as planar objects. On the other hand, the geometrical transformation and position of the feature points are lost with this simplification. Therefore, assumption $A_{o 3}$ is adopted. With this assumption, the global geometrical information can be retrieved by the function $f_{o b j}$. Thus, the planar representation of the object surface will be used at our feature point extraction process.

\subsection{Background Robust Features Extraction}

First, in order to extract feature points that are robust to background change, we apply a similar approach to (PILET; LEPETIT; FUA, 2008). However, here we render different background with the object in the same position and extract only features that appears several times. Here we render the object texture as a plane, as presented at 6.1, with randomly generated Perlin noise (AKENINE-MOLLER; MOLLER; HAINES, 2008) background, as shown at figure 17 .

Figure 17 - Perlin noise background used in this work, used to simulate different background conditions.
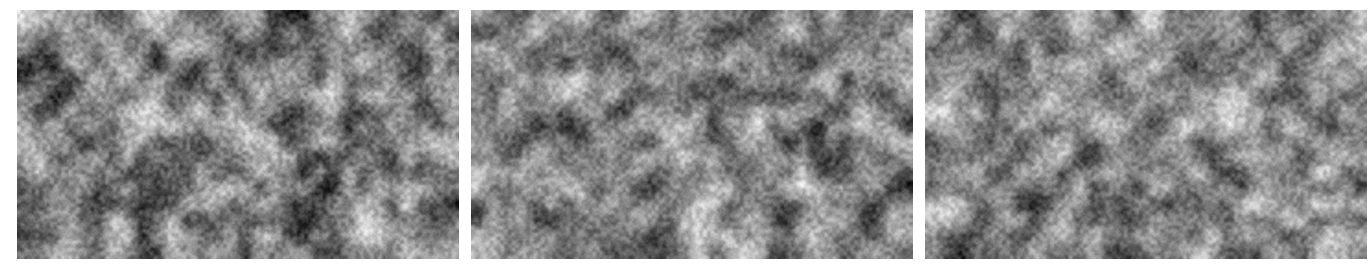

Source: Author.

The Perlin noise can be defined as a sequential sum of noises with different grids (or frequencies) with decaying amplitude, image 18 illustrates this process. This noise is particularly good as background in our work, since lot of natural textures can be simulated with this noise texture (AKENINE-MOLLER; MOLLER; HAINES, 2008), therefore used to simulate different types of random and natural backgrounds. 
Figure 18 - Perlin noise creation process. Consecutive noise adding and resizing creates the sequential sum of noises with different frequencies.

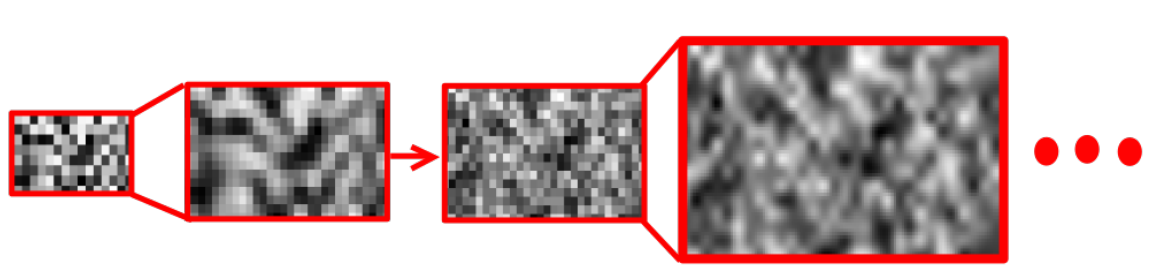

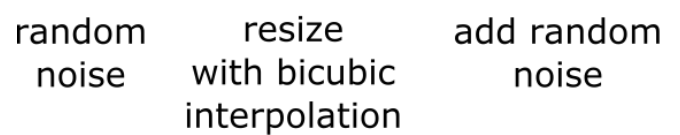

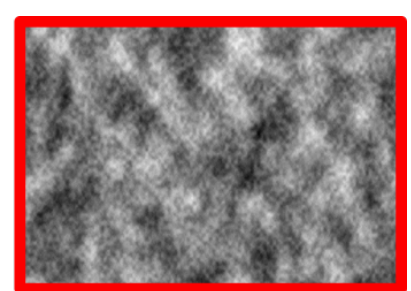

final image

Source: Author.

Here, $n_{\text {back }}$ number of images of the object in the same pose are rendered with different random backgrounds. Then, conventional SURF feature points extraction methods are applied on all the $n_{\text {back }}$ images, and only a set of $F_{v i e w}=\left[f_{v 0}, \ldots, f_{v n}\right]$ of feature points that appears in all the $n_{\text {back }}$ images are collected. With this process feature points that detected totally out of the object or composed by partially out of the object can be excluded, as illustrated by figure 33 at chapter 9 . This also means that all the features $F_{\text {view }}$ are totally contained inside the object surface and locally planar.

Each feature point $f_{v i}$ inside $F_{\text {view }}$ contains information of the feature point image space $2 \mathrm{D}$ position, $p^{I}$, its orientation $2 \mathrm{D}$ vector $\vec{o}$, scale $s$ and its descriptor desc. So, we have $f_{v i}=\left\{p_{i}^{I}, \vec{o}_{i}, s_{i}, \operatorname{desc}_{i}\right\}$.

\subsection{Affine Transformation Robust Features Extraction}

Also, we need to extract features that are robust to view transformations. For this purpose, we apply a method similar to ASIFT feature points presented byMorel e $\mathrm{Yu}$ (2009). In his work, Morel achieve fully affine invariant features, by transforming the reference image itself and the target image with different affine transformations, after this extracting and matching them to find the correlations. Here in this work, we apply a similar approach to ASIFT (MOREL; YU, 2009), extracting features across different affine transformations. However, unlike ASIFT, we apply the affine transformations only that the reference image.

First, the object texture image is rendered in different 3D poses with a known projection matrix $\mathbf{K}_{r}$, at known position $\mathbf{p}_{o b j}$ (typically at the center of the global coordinates), normal $\vec{n}_{o b j}$ and transformation matrix of camera coordinates $\mathbf{E}_{c a m}$. For each pose the object is rendered also with the different backgrounds approach as presented at section 6.2 . 
Here $\mathbf{K}_{r}$ is set to be identical with the projection matrix of the capturing system color camera projection matrix $\mathbf{K}_{r}=\mathbf{K}_{\text {color }}$, defined at chapter 4 . This enable more similar appearance of the features points the detection process. Also, this characteristic be explored at the local pose estimation with RGB cameras.

The object texture poses are chosen similarly to (MOREL; YU, 2009), such that object texture is maintained at the center of the coordinate system and the camera is positioned with different latitudes and zeniths angle values (as illustrated by our camera model in figure 19). The latitudes $\theta$ are selected according the rule $\theta=\arccos (1 / t)$ and $t$ are selected by $t=1, a^{1}, a^{2}, \ldots, a^{n_{t}}$ with $n_{\theta}=5$. However, unlike (MOREL; YU, 2009), we rendered the object with constant and equally spaced zeniths $\phi=2 \pi / u$ where $u=1, \ldots, n_{u}$ with $n_{u}=10$, in order to extract robust features across different affine transformations.

Figure 19 - Camera pose models.

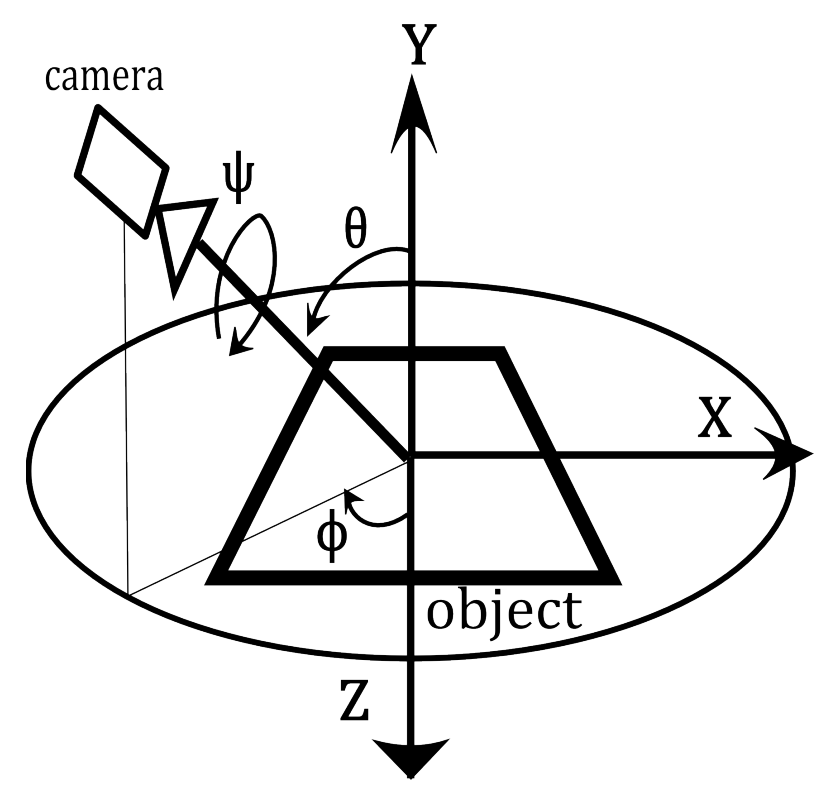

Source: Author.

Also, to be more robust across camera positions, the object is rendered with different camera distances $d_{c a m}=\left\{d_{0}, \ldots, d_{n_{d}}\right\}$ to the object. This is because, although the features as SURF (BAY et al., 2008) and SIFT (LOWE, 1999) are scale invariant, some features can appear or change slightly across different camera distances and the reference image resolutions.

For each camera pose $i$ image with known camera transformation $\mathbf{E}_{c a m}^{i}$, we extract the $F_{\text {view }}^{i}$ set of background robust feature points as presented at 6.2. Then, all the $F_{v i e w}^{i}$ are compared to themselves using feature matching methods as in (LOWE, 2004). With this matching process, we list $F_{o b j_{V}}=\left\{F_{o b j}^{0}, \ldots, F_{o b j}^{n}\right\}$ feature points, where each member $F_{o b j}^{i}$ are feature point collection matched to be similar within a threshold of $\tau_{o b j}$.

$F_{o b j}^{i}$ is a collection of information pair $F_{o b j}^{i}=\left\{\left[f_{v 0}, \mathbf{E}_{c a m}^{0}\right], \ldots,\left[f_{v j}, \mathbf{E}_{c a m}^{j}\right], \ldots,\left[f_{v n}, \mathbf{E}_{c a m}^{n}\right]\right\}$. 
Each pair $\left[f_{v j}, \mathbf{E}_{c a m}^{j}\right]$ contains the information of feature point $f_{v j}$ found at the image with camera view transformation $\mathbf{E}_{c a m}^{j}$.

This matching process means that each feature point $f_{v j}$ within $F_{o b j}^{i}$ are the same point at the object texture surface $I_{t e x}$. Also meaning that $F_{o b j_{V}}$ is a collection of feature points that are different of each other, and for each feature point, the information of the observed image and information of how the feature point itself changes are listed across the found camera transformations.

One simple way to filter this collection and find a limited set of feature points $F_{o b j}$ is to list feature points that appears in more than $n_{v}$ views. With this, feature points $F_{o b j}=F_{o b j_{V}}\left(\right.$ length $\left.\left(F_{o b j_{V}}\right)>n_{v}\right)$, that are robust across at least $n_{v}$ views, are filtered in order to be used at the pose estimation.

The algorithm 1 and 2 presents this entire process of view and background robust feature extraction process.

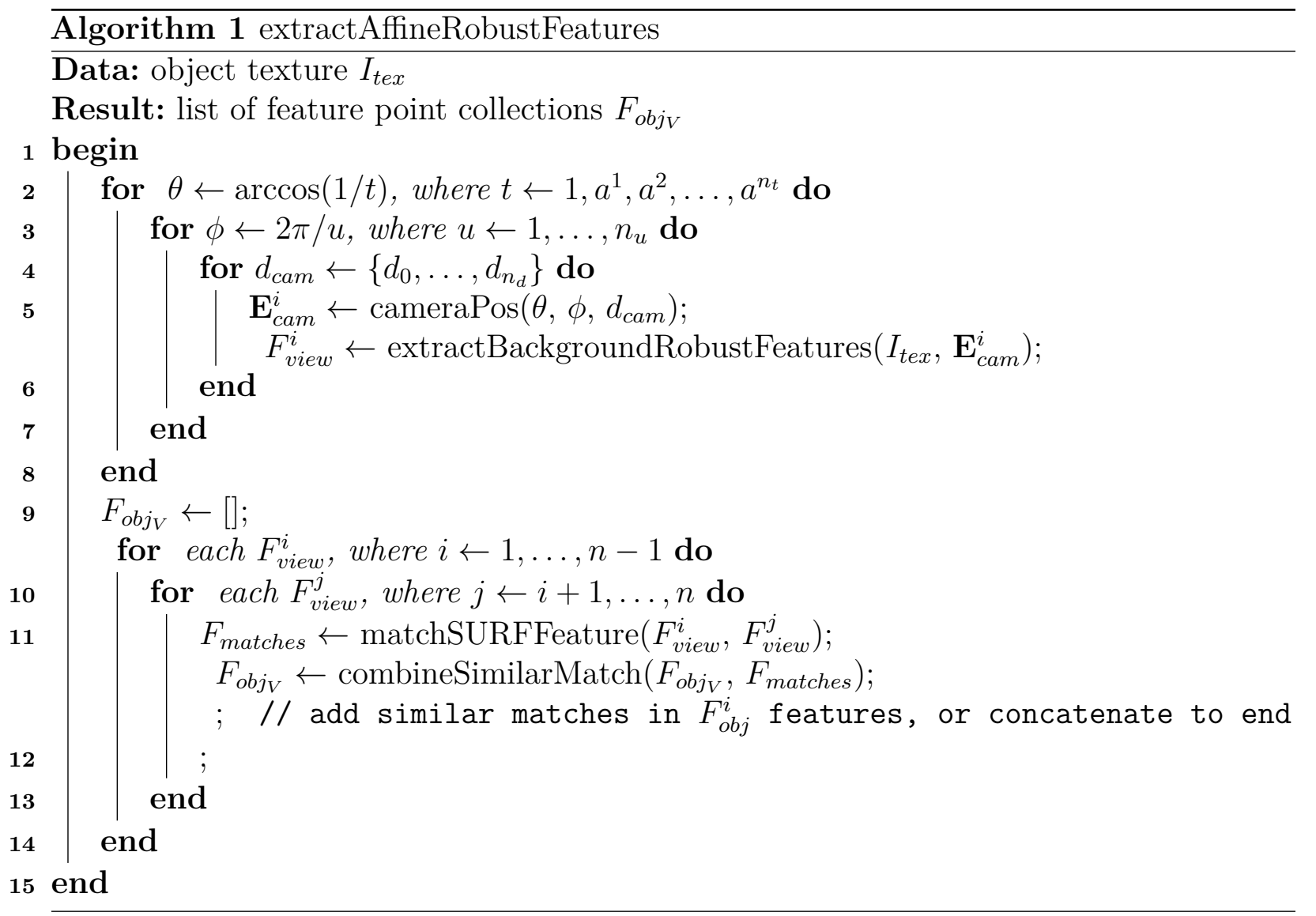

Where, extractSURFFeatures and matchSURFFeature represents the method of feature points extraction (BAY et al., 2008) and matching (LOWE, 2004). RandomPerlinBackground represents the generation of Perlin noise background presented in 6.2, extractBackgroundRobustFeatures is the method presented in algorithm 2, and combineSimilarMatch combines the two groups into a longer group which contains both $F_{o b j_{V}}$ and $F_{\text {matches }}$. 


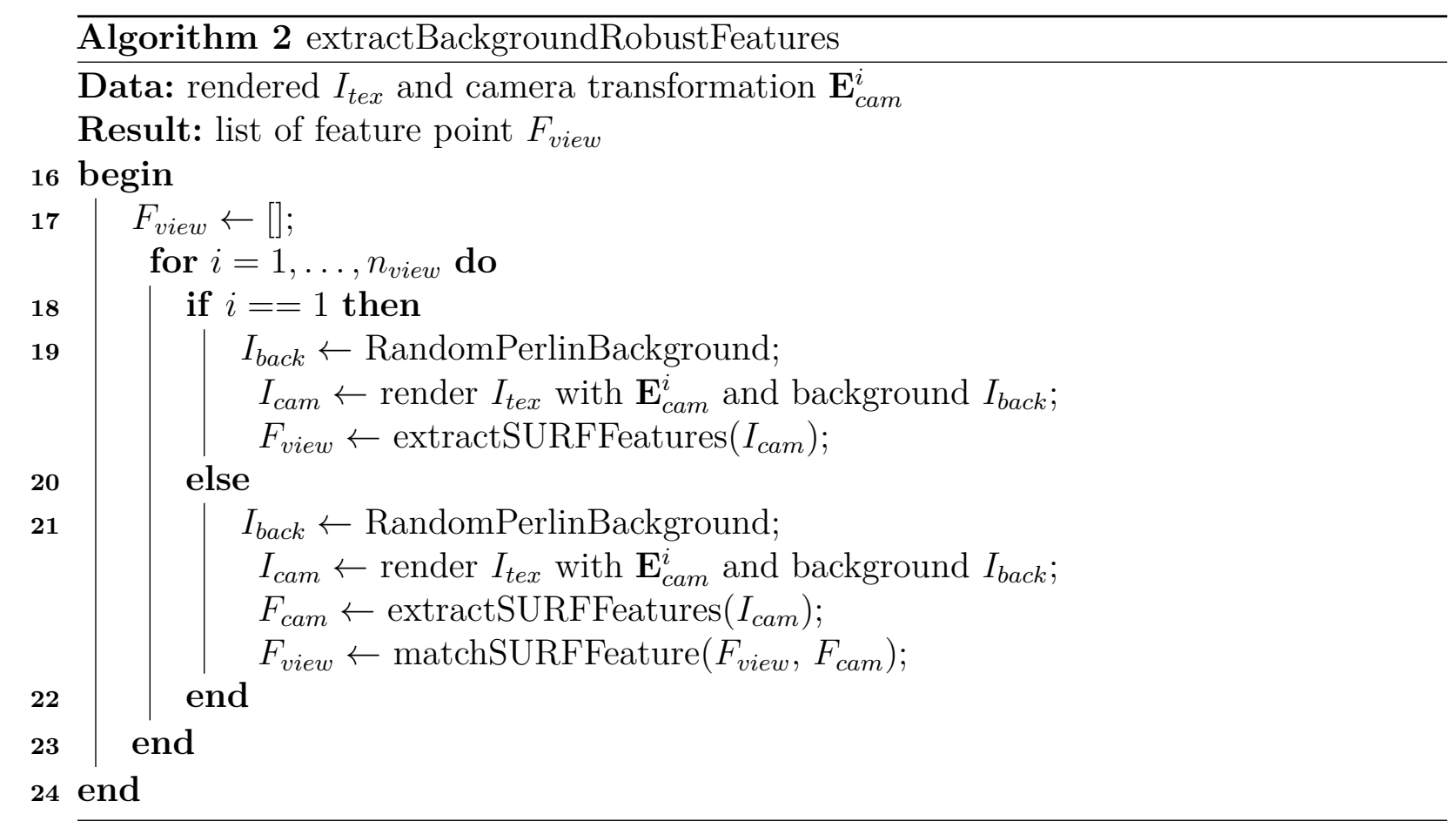

\subsection{Entropy Maximization Feature Point Selection Method}

At the section above, we proposed a method to filter feature points lists $F_{o b j_{V}}$ by finding $F_{o b j}^{i}$ that are found in more then $n_{v}$ view position. However, this can list feature points that are viewed in same camera transformations, since features that are view in lower $\theta$ (perpendicular to the image plane) are more found across near views. This can lead to feature points list that are only find in limited views, making the object observed only in this limited views, instead all the possible cases, as illustrated by figure 20a.

Figure 20 - Converage area of feature points. Each color area represents the coverage area that a single feature can be observed. If only total area is considered, it can generate several feature points that are visible in the same area and not covering others. Our entropy maximization method tries to maximize the total observation area.

(a) Selecting only feature points with bigger coverage area.

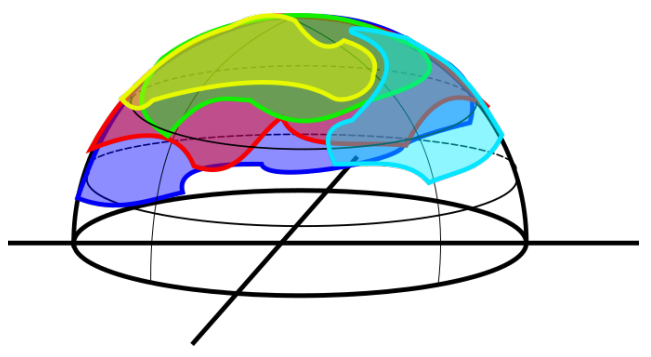

(b) Selecting feature points to maximize the total area by maximizing the observation entropy.

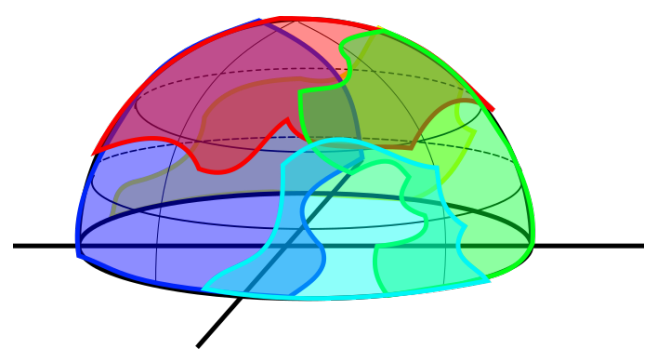

Source: Author. 
Here we also propose a different method for filtering $F_{o b j_{V}}$ by maximizing the entropy of the feature points found views. Our objective here is to list feature points that cover maximum visibility of the object across different perspective views, as shown at figure 20b.

For this purpose, for each newly add feature to the list $F_{o b j}$, we try to find the feature $F_{o b j}^{i}$ within $F_{o b j_{V}}$ that maximize the equation:

$$
F_{o b j 2 a d d}=\underset{F_{o b j}^{i}}{\operatorname{argmax}} H\left(P\left(O_{o v^{j}}\right)\right),
$$

where $H\left(P\left(O_{v o}\right)\right)$ is the entropy function () of the probability to found the object in some view $j, P\left(O_{o v^{j}}\right)$. This probability can be defined as a joint probability $P\left(O_{o v^{j}}\right)=P\left(O_{v^{j}} \cdot O_{o}\right)$, where $O_{o}$ is the event of the feature been fount, and $O_{v^{j}}$, the object to be fount at that position $j$. By Bayesian rule (), this probability can be calculated as:

$$
P\left(O_{v^{j}} . O_{o}\right)=P\left(O_{v^{j}} \mid O_{o}\right) \cdot P\left(O_{o}\right)
$$

Once the view space was discretized by the $\theta$ and $\phi$ and $d_{\text {cam }}$ view, probability of the object been found at some view $j, P\left(O_{v^{j}} \mid O_{o}\right)$, can be calculated as

$$
P\left(O_{v^{j}} \mid O_{o}\right)=\frac{n_{v}^{j}}{n_{\text {total }}}
$$

where $n_{v}^{j}$ are the total observations at view $j$ and $n_{\text {total }}$ is the total of observations in all views with the feature $F_{o b j}^{i}$ added. Also, probability of this feature been found $P\left(O_{o}\right)$ can be calculated as

$$
P\left(O_{o}\right)=\frac{n_{f t}}{n_{\text {totalviews }}}
$$

where $n_{f t}$ is the total number of views that $F_{o b j}^{i}$ is seen and $n_{\text {totalviews }}$ is the total number of possible views (in this case $n_{\text {totalview }}=n_{u} * n_{t} * n_{d}$, where $n_{u}$ is total number of $\phi$ views, $n_{t}$, total number of $\theta$ and $n_{d}$ total number of distances $d$ ).

With these probabilities and the entropy equation for information theory (), equation 6.1 can be rewrite as

$$
F_{o b j 2 a d d}=\underset{F_{o b j}^{i}}{\operatorname{argmax}}-\sum_{j}\left(P\left(O_{v^{j}} \mid O_{o}\right) \cdot P\left(O_{o}\right)\right) \log \left(P\left(O_{v^{j}} \mid O_{o}\right) \cdot P\left(O_{o}\right)\right) .
$$

For each new feature point to be listed, we re-calculate the entropy as in 6.5, and then add this point to the list of robust feature points to be used in the next steps. This process is stopped until a number $n$ of desired feature points are extracted.

\subsection{Chapter Conclusion}

In this chapter we have presented the feature points extraction method proposed in this work. This feature points extraction is a crucial step for the object pose estimation, 
once the probability of the object to be found in a new frame is directly related with the quality of the feature points extracted. Here we propose a modification in the ASIFT () method, in order to extract background and camera transformation robust features.

At this point, we have extracted the feature point list

$$
F_{o b j}=\left\{\left[f_{o b j}^{0}, E_{v i e w^{0}}\right], \ldots,\left[f_{o b j}^{n}, E_{v i e w^{n}}\right]\right\}
$$

Each feature point $f_{o b j}^{i}$ contains information of the feature point image space 2D position, $p^{I}$, its orientation $2 \mathrm{D}$ vector $\vec{o}$, scale $s$ and its descriptor desc $\left(f_{v i}=\left\{p_{i}^{I}, \vec{o}_{i}, s_{i}, \operatorname{desc}_{i}\right\}\right)$ and, for each feature point, the information of the camera transformation $E_{v_{i e w}}$ of the rendered image that this feature point was found is also collected. 


\section{Local Pose Estimation based on RGB-D In- formation}

Figure 21 - Information flow of our pose estimation approach based on RGB-D information.

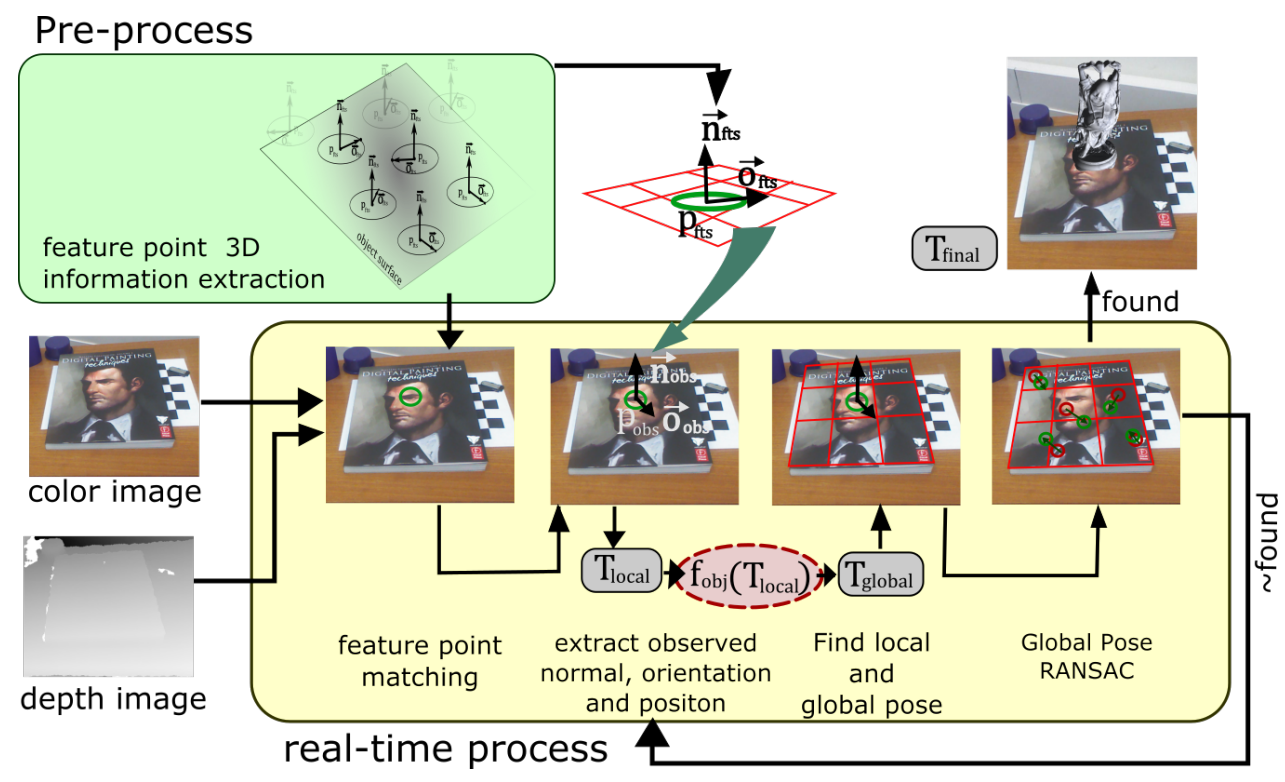

Source: Author.

In this chapter we present one of the core contributions of this work, the method applied to find the feature points local poses based on RGB-D capturing setup. Here, the local pose estimation is separated in two parts, the pre-process of object feature points, applied to extract the feature point spacial information, and the observed feature point local pose estimation process, which estimate the local pose of the feature point, in real-time, in a new image frame set $\left[I_{\text {color }}, I_{\text {depth }}\right]$. The information flow on this approach is presented in figure 21 .

In both parts, we consider that information of feature points $F_{o b j}$, from feature extraction (chapter 6) is already processed and known, also with the information and images of the object texture. As well as, capturing system and object information (camera projection matrix $K_{\text {color }}$, color image $I_{\text {color }}$ and depth map $I_{\text {depth }}$, object texture $I_{\text {gtex }}$ and mapping function $f_{o b j}\left(T_{t e x}\right)$, chapter 4$)$ are also considered to be known.

\subsection{Pre-process of Object Feature Points}

The goal of this first part is to collect spacial information of the feature points, extracted by the method of chapter 6 . This information will me matched at the pose 
estimation part in order to calculate the full transformation matrix of the feature point.

First, in order to collect these spatial information, for each extracted feature point within $F_{o b j}$, the $3 \mathrm{D}$ position of feature point point $\mathbf{p}_{f t s}$ is calculated. Once the object surface can be transformed to a plane as done in 6 , by assumption $A_{o 2}$ (chapter 4 ), we use plane line intersection method between the rendered object texture plane with the line that crosses the camera position $\mathbf{p}_{\text {cam }}$ and the feature point $3 \mathrm{D}$ position $\mathbf{p}^{w}$ detected at $2 \mathrm{D}$ position $p_{f t s}^{i m g}$ of the rendered image plane. Figure 22 illustrate this process.

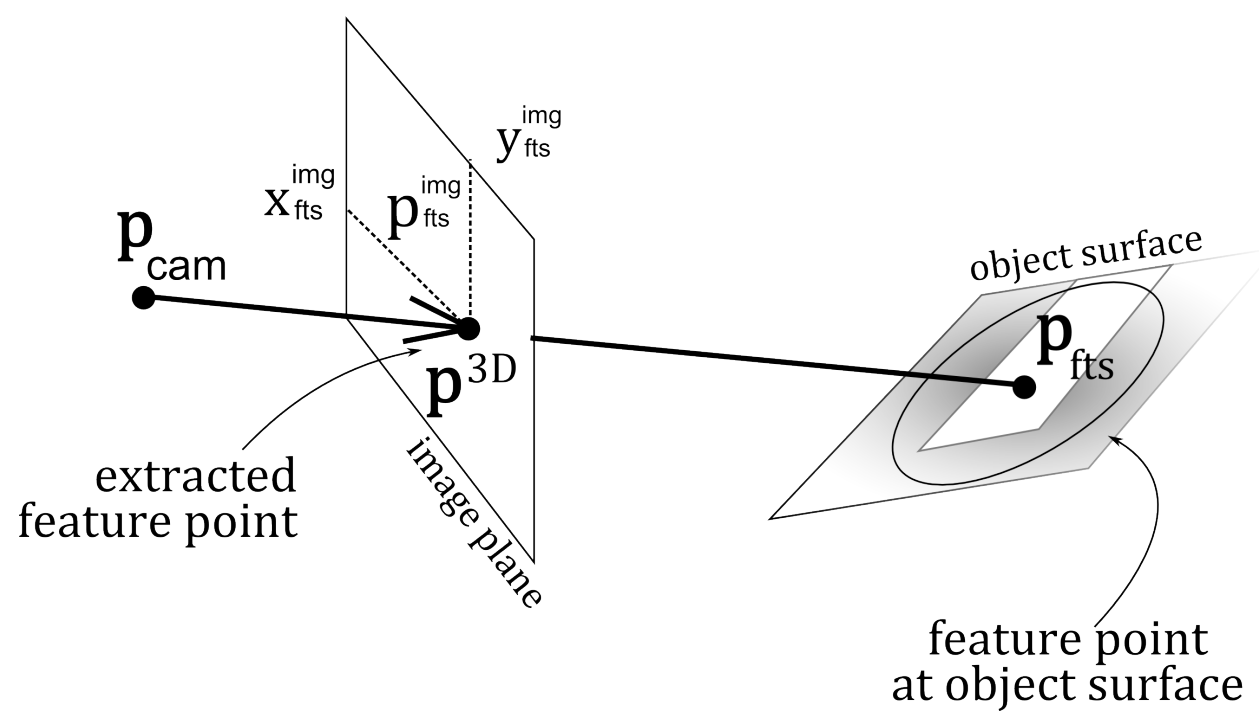

Figure 22 - Calculation of $\mathbf{p}_{\text {fts }}$ by $K_{\text {cam }}, \mathbf{p}_{\text {cam }}, \mathbf{p}^{3 D}$.

Once the projection matrix converts the homogeneous coordinates $3 \mathrm{D}$ point to the projection image plane, we have

$$
\begin{array}{r}
\mathbf{p}^{I}=\mathbf{K}_{\text {color }} \cdot \mathbf{p}^{H} \\
\mathbf{p}^{w}=\left[X^{3 D}, Y^{3 D}, Z^{3 D}\right]^{T} \\
\mathbf{p}^{H}=\left[X^{3 D} / Z^{3 D}, Y^{3 D} / Z^{3 D}, 1\right]^{T},
\end{array}
$$

where, $\mathbf{p}^{H}$ is the homogeneous form of $\mathbf{p}^{3 D}$. With this equation, by using the inverted projection matrix $\mathbf{K}_{\text {color }}^{-1}$, this $3 \mathrm{D}$ position related to the feature point can be calculated as

$$
\mathbf{p}^{w}=\mathbf{E}_{\text {cam }}^{-1} \cdot\left(z_{\text {img }} \cdot\left(\mathbf{K}_{\text {color }}^{-1} \mathbf{p}^{I}\right)\right)
$$

, where $z_{i m g}$ is some distance defined as distance of the image plane to the camera position (in this case can be set to 1 ), and $E_{c a m}$ is the rendered camera transformation matrix.

After this point $\mathbf{p}^{w}$ is calculated, in order to calculate $\mathbf{p}_{f t s}$, the intersection of the ray formed by $\left\{\mathbf{p}_{\text {cam }},\left(\mathbf{p}_{3 D}-\mathbf{p}_{\text {cam }}\right)\right\}$ and the plane of the image texture $I_{\text {tex }}$. As in chapter 6 , the object is placed at position $\mathbf{p}_{o b j}$ with normal $\vec{n}_{o b j}$, once the same information is been used. Therefore, the position $\mathbf{p}_{\text {fts }}$ can be calculated as the image 23 shows. 


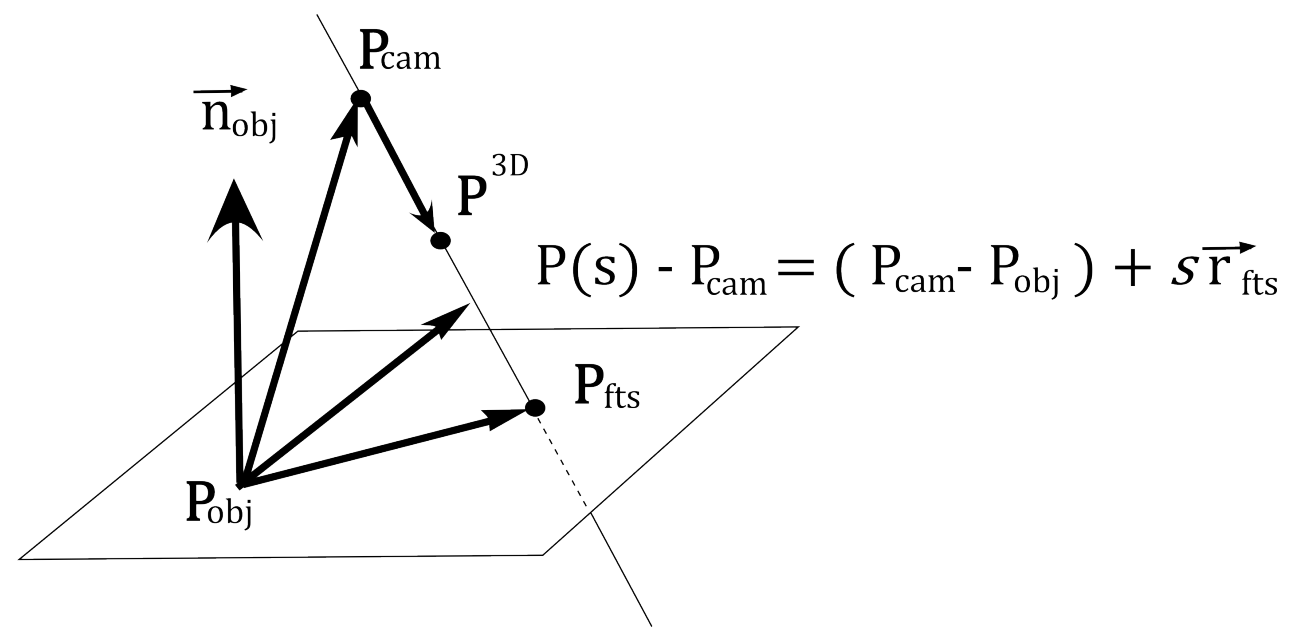

Figure 23 - Calculation of $\mathbf{p}_{f t s}$ by plane intersection.

Here, the position $\mathbf{p}_{f t s}$ can be calculated when $\mathbf{p}(s)$ is orthogonal to the plane normal $\vec{n}_{o b j}, \mathbf{p}(s)=\left(\mathbf{p}_{c a m}-\mathbf{p}_{o b j}\right)+\mathbf{p}_{c a m}+s \vec{r}_{f t s}=0$, giving us the equations

$$
\begin{array}{r}
\mathbf{p}_{f t s}=\mathbf{p}_{c a m}+s \vec{r}_{f t s} \\
s=\frac{\vec{n}_{o b j} \cdot\left(\mathbf{p}_{o b j}-\mathbf{p}_{c a m}\right)}{\vec{n}_{o b j} \cdot \vec{r}_{f t s}} \\
\vec{r}_{f t s}=\left(\mathbf{p}^{w}-\mathbf{p}_{c a m}\right)
\end{array}
$$

to calculate the position of the feature point.

After calculating the $3 \mathrm{D}$ position, a $3 \mathrm{D}$ vector $\vec{o}_{f t s}$ over the texture image plane that points toward the feature $2 \mathrm{D}$ orientation is calculated. This is done by calculating the three-dimensional position of point $\mathbf{p}_{o}$ in a similar way of calculating $\mathbf{p}_{f t s}$, but instead of calculating using the rendered image position $\mathbf{p}^{I}$ of the feature, we use the $2 \mathrm{D}$ position

$$
\mathbf{p}_{o}^{I}=\mathbf{p}^{I}+s_{f t s} \vec{v}_{\text {ori }}
$$

where $s_{f t s}$ is the size of the feature patch that was used to extract the feature point descriptor, and $\vec{v}_{\text {ori }}$ is the two-dimensional orientation vector of the feature. Figure 24 illustrates this vector and the calculation process.

With this, $\vec{o}_{f t s}$ is calculated as

$$
\vec{o}_{f t s}=\left(\mathbf{p}_{o}-\mathbf{p}_{f t s}\right) /\left\|\left(\mathbf{p}_{o}-\mathbf{p}_{f t s}\right)\right\|
$$

Finally, the normal of the feature $\vec{n}_{f t s}$ is calculated. Once the object is converted into a planar image, the feature point normal is the same as that of the plane normal, $\vec{n}_{f t s}=\vec{n}_{\text {plane }}$.

This process is repeated for each feature point within $F_{o b j}$ and the information of position $\mathbf{p}_{f t s}$, orientation vector $\vec{o}_{f t s}$ and normal $\vec{n}_{f t s}$ are added to the feature point. For implementation purposes, the feature point extraction and feature point local spacial 


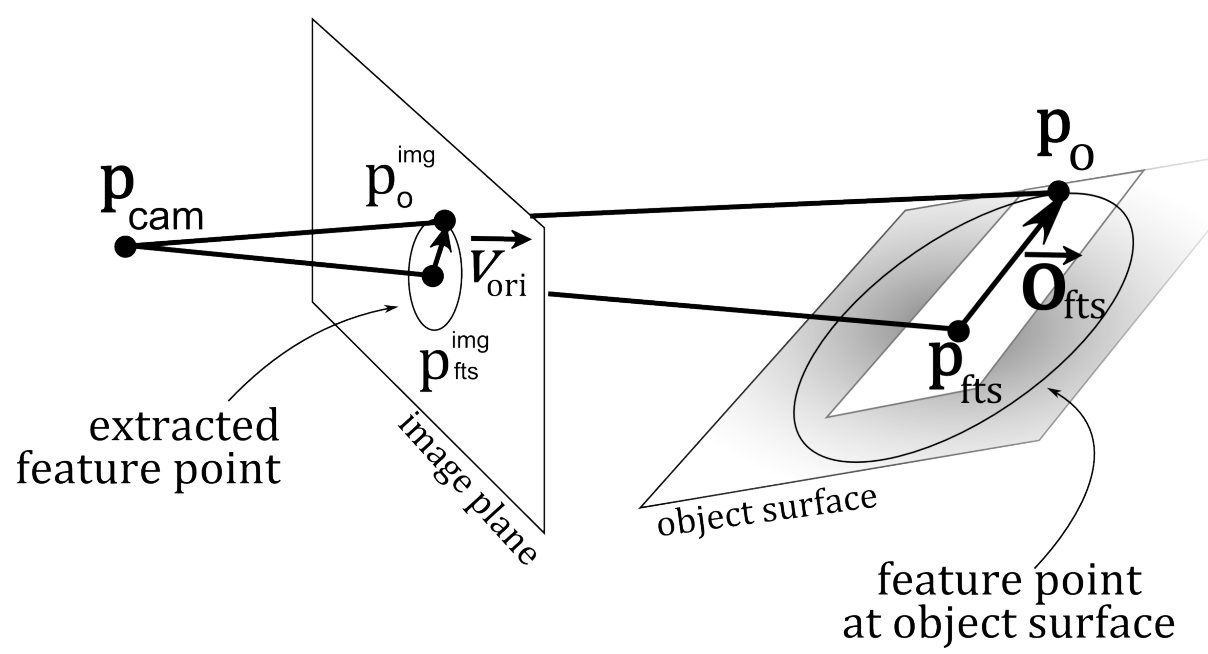

Figure 24 - Calculation of $\vec{v}_{\text {ori }}$.

information can be processed at the same time, when they are listed at the process shown at section 6.3. At this part each feature point, $f_{o b j}^{i}=\left\{\mathbf{p}_{f t s}^{i}, \vec{n}_{f t s}^{i}, \vec{o}_{f t s}^{i}, d s c_{f t s}^{i}\right\}$, contains three-dimensional information of position $\mathbf{p}_{f t s}^{i}$, normal $\vec{n}_{f t s}^{i}$, orientation vector $\vec{o}_{f t s}^{i}$ and its descriptor $d s c_{f t s}^{i}$. Figure 25 illustrates these information.

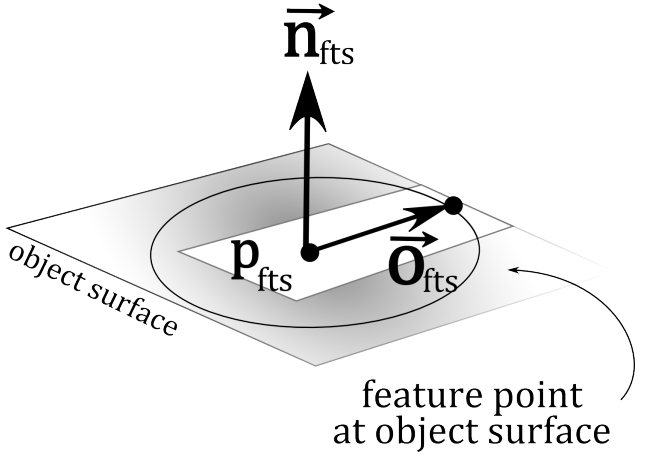

(a) feature point informations

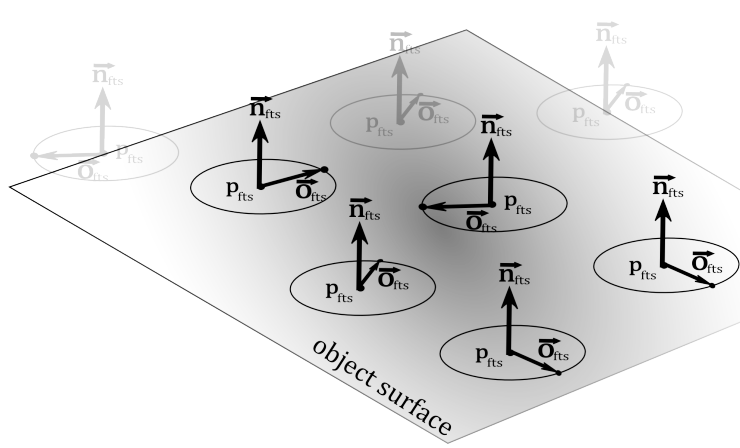

(b) feature points over object surface

Figure 25 - Illustration of the feature points collected information. At right, information of a single feature point; at left, illustration of how are the local information spread over the object surface texture at this point.

As a last task of this pre-process, all the calculated 3D information are reconverted to match the original object using functions $f_{\text {obj }}\left(T_{t e x}\right)=T_{\text {global }}$. In case of planar objects, these information do not change, but for 3D rigid objects, these information are reconverted so they lie over the object surface. This process can be also done after at the pose estimation part, however it will create more computational process to be done at the real-time part. In order to avoid this extra process, it is better to be done at his pre-process part. 


\subsection{Observed Feature Point Local Pose Estimation}

In order to estimate the local pose of the feature point using RGB-D information, initially, feature points are extracted from the newly captured RGB frame $I_{\text {color }}$ and compared with $F_{o b j}$ using the conventional feature match method. For each match feature point, the three-dimensional position $\mathbf{p}_{\text {obs }}$ at the surface of the object that generated the feature is calculated from its two-dimensional position $p_{f}$ and depth $d$, obtained from the depth map $D_{\text {cap }}$, as

$$
\mathbf{p}_{\text {obs }}=d\left(K_{\text {color }}^{-1} \mathbf{p}_{f t s}^{I}\right)
$$

by using the inverse of the projection matrix as in section 7.1.

Then, similarly to (7.4), using the size of patch $s_{f t s}$ and two-dimensional orientation vector $\vec{v}_{\text {ori }}$ returned from the feature extraction, three-dimensional position $\mathbf{p}_{o}$ of the object seen by the RGB frame at 2D position

$$
p_{o}^{I}=p_{f t s}^{I}+s_{f t s} \vec{v}_{\text {ori }}
$$

is calculated. From $\mathbf{p}_{o}$, obtained by $\mathbf{p}_{o}^{I}$ and its depth value, the vector that points toward the feature orientation over the object surface

$$
\vec{o}_{o b s}=\frac{\mathbf{p}_{o}^{w}-\mathbf{p}_{f t s}^{w}}{\left\|\mathbf{p}_{o}^{w}-\mathbf{p}_{f t s}^{w}\right\|}
$$

is calculated.

Once the feature is considered to be locally planar by assumption $A_{o 2}$, the cross product of any two non-collinear vectors that belong to the feature patch gives us the normal of the feature point $\vec{n}_{o b s}$. Thus, we calculate the feature normal by the cross product of $\vec{o}_{o b s}$ with another vector over the observed surface. In order to guarantee that both vectors are not collinear, a 3D point $\mathbf{p}_{\text {ort }}^{w}$ over the surface is calculated similarly to (7.7) by the $2 \mathrm{D}$ point of the RGB frame at position $p_{\text {ort }}^{I}=\mathbf{p}_{f}^{I}+s \vec{v}_{\text {ort }}$, where, $\vec{v}_{\text {ort }}$ is a vector in the RGB frame coordinates, orthogonal to $\vec{v}_{\text {ori }}$. Then, we calculate $\vec{n}_{\text {obs }}$ as

$$
\vec{n}_{o b s}=\vec{o}_{o b s} \times\left(\frac{\mathbf{p}_{o r t}^{w}-\mathbf{p}_{f t s}^{w}}{\left\|\mathbf{p}_{o r t}^{w}-\mathbf{p}_{f t s}^{w}\right\|}\right)
$$

where $\mathbf{p}_{\text {ort }}^{w}$ is the point over the object surface calculated by $p_{\text {ort }}^{I}$ and its depth value.

With $\mathbf{p}_{f t s}, \vec{o}_{o b s}$ and $\vec{n}_{o b s}$, it is possible to calculate the pose of the feature point $\mathbf{T}_{f t s}$ similarly to (HORN, 1987) and (CHOI; CHRISTENSEN, 2012). Here, we calculate this transformation as a combination of 3 transform matrices $\mathbf{T}_{r 1}, \mathbf{T}_{r 2}$ and $\mathbf{T}_{t}$. First, $\mathbf{T}_{r 1}$ rotates the original feature normal $\vec{n}_{f t s}$ to match with $\vec{n}_{o b s}$. This is done by a transformation that rotates around axis $\vec{r}_{a 1}$ and angle $a_{1}$ as

$$
\begin{array}{r}
\vec{r}_{a 1}=\vec{n}_{o b s} \times \vec{n}_{f t s} \\
a_{1}=\arccos \left(\vec{n}_{o b s} \cdot \vec{n}_{f t s}\right)
\end{array}
$$


Then $\mathbf{T}_{r 2}$ rotates the new coordinate system to match $\mathbf{T}_{r 1} \vec{o}_{f t s}$ and $\vec{o}_{o b s}$. As (7.10), $\mathbf{T}_{r 2}$ is calculated as a rotation transformation with axis $\vec{r}_{a 2}$, and angle $a_{2}$ as:

$$
\begin{array}{r}
\vec{r}_{a 2}=\vec{o}_{o b s} \times\left(\mathbf{T}_{r 1} \cdot \vec{o}_{f t s}\right) \\
a_{2}=\arccos \left(\vec{o}_{o b s} \cdot\left(\mathbf{T}_{r 1} \cdot \vec{o}_{f t s}\right)\right) .
\end{array}
$$

Finally $\mathbf{T}_{t}$ is a translation of the coordinate system to the observed point $\mathbf{p}_{\text {obs }}$, calculated as

$$
\vec{t}=\mathbf{p}_{o b s}-\mathbf{T}_{r 2} \cdot \mathbf{T}_{r 1} \cdot \mathbf{p}_{f t s}
$$

The final transformation matrix of the feature pose $\mathbf{T}_{f t s}^{i}$ is given by

$$
T_{f t s}^{i}=\left(\mathbf{T}_{t} \cdot \mathbf{T}_{r 2} \cdot \mathbf{T}_{r 1}\right)
$$

This calculation is repeated for all the match features between the new feature and pre-processed features $F_{o b j}$. With this process, we extract all the match feature points local poses $\mathbf{T}_{f t s}=\left\{\mathbf{T}_{f t s}^{0}, \ldots, \mathbf{T}_{f t s}^{n}\right\}$.

Also, if the object is a rigid 3D object, we need rectify those local transformation using $f_{o b j}$ (presented at section 4.1), in order to obtain the right transformation of to the object space. Obtaining the true object transformation as

$$
\mathbf{T}_{f t s O b j}=f\left(\mathbf{T}_{f t s}\right) .
$$

\subsection{Global Pose Estimation based on Local Poses}

After calculating the local poses of feature points $\mathbf{T}_{\text {local }}$, these information are processed in order to calculate the global pose of different classes of object. In section 7.3.1 we present a method based on RANSAC for rigid objects pose estimation. Also, in section 7.3.2, we present the method applied in this work, in order to obtain rough deformable object registration for $\mathrm{AR}$.

\subsubsection{Planar and Rigid Objects}

As presented in 5.1, the fact that all the points has the same transformation, also means that we can find the object pose by one pose of one point of the object. This characteristic lead us to one of the results of our local pose based approach. That, unlike other state-of-art methods that needs at least three points (LIMA et al., 2012; LEPETIT; MORENO-NOGUER; FUA, 2009; HENRY et al., 2012), we can find the rigid object pose by using only one match feature point. These results are presented at the section 9.2.2.

However, only one observation leads to weak pose estimation, that can be easily affected by noises. Therefore, we propose a methods based on multiple local pose information. 
This global pose based on multiple local poses could be processed by all the extracted local poses. However, this process could also be computationally heavy.

In order to achieve process time near real-time systems, we also applied a variant of RANSAC approach (FISCHLER; BOLLES, 1981) to our global pose estimation. As Fischler e Bolles (1981), initially a randomly selected subset $S 1$ of the found feature points is selected by a percentage $p_{\text {subset }}$. Then, pose transformation matrices $\mathbf{T}_{\text {local }}^{r}$ within $\mathbf{T}_{\text {local }}$ are randomly selected.

For each point within $S 1$, the quadratic error $\epsilon^{i r}$ of $\mathbf{T}_{\text {local }}^{r}$

$$
\epsilon^{i r}=\left\|\mathbf{T}_{l o c a l}^{r} \cdot \mathbf{p}_{f t s}^{i}-\mathbf{p}_{o b s}^{i}\right\|^{2}
$$

is calculated as the distance between the observed positions of the feature in $S 1$ and its estimated position based on $\mathbf{T}_{f}^{r}$. Then, the number of points $n_{\tau}$ that have distance error less than a threshold $\tau_{\text {error }}$ are counted.

However, unlike Fischler e Bolles (1981), we do not stop the search after any $\mathbf{T}_{\text {local }}^{r}$ that has $n_{\tau}$ larger then a percentage $p_{\text {found }}$ of $S 1$. Instead, we keep the search until we find $n_{\text {mean }}$ transformations $\mathbf{T}_{R A N S A C}=\left\{\mathbf{T}_{0}, \ldots, \mathbf{T}_{n_{\text {mean }}}\right\}$ that satisfy $\left(n_{\tau}>p_{\text {found }} \cdot p_{\text {subset }} \cdot n\right)$. Then, these transformations are converted to a set of vectors of translation $\vec{t}_{f}$ and quaternions $q_{f}^{i}(\mathrm{HORN}, 1987)$. Finally, the object global position $\mathbf{T}_{o b j}$ is calculated as the transformation matrix obtained by the transform resulting from the mean of the translations vectors $\vec{t}_{f}$ and quaternions $q_{f}$.

This change is due to the nature of error returned by our approaches. In case of local pose estimation method at 7 , since $\vec{o}_{f t s}$ and $\vec{o}_{o b s}$ are both calculated by the projection of the object surface texture at the captured image, small projection errors at the image of the feature point orientation vector can happen, as can be seen at Figure 39.

In case the RANSAC method can not find $n_{\text {mean }}$ samples of valid transformations in $N_{\text {trial }}$ trials, our global pose detection method returns the transformations $\mathbf{T}_{o b j}$ that have the minimal error within the sampled transformation of the $N_{\text {trial }}$. This transformation is the one that minimizes the sum of the quadratic errors, calculated by

$$
\mathbf{T}_{o b j}=\underset{\mathbf{T}_{\text {local }}^{r}}{N_{\text {trial }}} \sum_{i=1}^{n}\left(\left\|\mathbf{T}_{f} \cdot \mathbf{p}_{f t s}^{i}-\mathbf{p}_{o b j}^{i}\right\|^{2}\right) .
$$

However, with a small subset of $\mathbf{T}_{\text {local }}^{r}$, within $N_{\text {trial }}$. Algorithm 3 illustrates this process.

In order to achieve even faster process times, this RANSAC method can also be implemented within the process of local pose estimation, presented at sections 7.2, without calculating all the local poses first to achieve the best pose. This is done by calculating the $\epsilon^{i}$ error for each calculated local pose, and finding if this pose satisfy all the conditions shown above. Algorithm 4 illustrates this altered process. 
Algorithm 3 Global Pose by RANSAC with full list of $\mathbf{T}_{\text {local }}$

Data: Local poses $\mathbf{T}_{\text {local }}$, pre-processed feature points $F_{o b j}$, observed feature points $3 \mathrm{D}$ position list $\mathbf{p}_{o b j}$, point subset percentage $p_{\text {subset }}$, acceptance percentage of correct observations $p_{\text {found }}$, error threshold $\tau_{\text {error }}$, number of samples to mean $n_{\text {mean }}$, number of trials $N_{\text {trial }}$

Result: Object transformation $\mathbf{T}_{o b j}$

25 begin

$26 \quad p_{\text {subset }} \leftarrow\left\{\mathbf{p}_{o b j}^{0}, \ldots, \mathbf{p}_{\text {obj }}^{n_{\text {subset }}}\right\}$ listed as a random subset of $\mathbf{p}_{\text {obj }}$ with $p_{\text {subset }}$ percentage of total observed points;

found $\leftarrow$ false;

$n_{\text {trials }} \leftarrow 0$;

while $($ found $==$ false $) \&\left(n_{\text {trials }}<N_{\text {trials }}\right)$ do

28

29

30

31

32

33

34

35

36

37

38

39

40

41

42

43

44

$n_{\text {correct }} \leftarrow 0$;

$\mathbf{T}_{\text {local }}^{r} \leftarrow$ random sample within $\mathbf{T}_{\text {local }}$;

for all elements within $p_{\text {subset }}$ do

$\epsilon^{i} \leftarrow\left\|\mathbf{T}_{\text {local }}^{r} \cdot \mathbf{p}_{\text {fts }}^{i}-\mathbf{p}_{\text {obs }}^{i}\right\|^{2}$;

if $\epsilon^{i}<\tau_{\text {error }}$ then

$\mid n_{\text {correct }} \leftarrow n_{\text {correct }}+1$;

end

end

if $n_{\text {correst }}>n_{\text {subset }} \cdot p_{\text {found }}$ then

found $\leftarrow$ true;

$\mathbf{T}_{o b j} \leftarrow \mathbf{T}_{l o c a l}^{r}$

else

$n_{\text {trials }} \leftarrow n_{\text {trials }}+1$

if minError $>\sum \epsilon^{i}$ then

minError $\leftarrow \sum \epsilon^{i}$;

$\mathbf{T}_{\text {min }} \leftarrow \mathbf{T}_{\text {local }}^{r}$

end

end

end

if found $\neq$ true then

$\mathbf{T}_{o b j} \leftarrow \mathbf{T}_{m i n}$

end

return $\mathbf{T}_{o b j}$;

end 
Algorithm 4 Global Pose by RANSAC calculated in parallel with $T_{\text {local }}^{i}$

Data: pre-processed feature points $F_{o b j}$, feature points list $F_{o b s}$ observed in new frame, point subset percentage $p_{\text {subset }}$, acceptance percentage of correct observations $p_{\text {found }}$, error threshold $\tau_{\text {error }}$, number of samples to mean $n_{\text {mean }}$, number of trials $N_{\text {trial }}$

45
Result: Object transformation $\mathbf{T}_{o b j}$

\section{begin}

calculate all 3D position $\mathbf{p}_{o b j}$ of all $F_{o b s}$

$p_{\text {subset }} \leftarrow\left\{\mathbf{p}_{\text {obj }}^{0}, \ldots, \mathbf{p}_{\text {obj }}^{n_{\text {subset }}}\right\}$ listed as a random subset of $\mathbf{p}_{\text {obj }}$ with $p_{\text {subset }}$ percentage of total observed points;

found $\leftarrow$ false;

$n_{\text {trials }} \leftarrow 0$;

while $\left(\right.$ found $==$ false) $\&\left(n_{\text {trials }}<N_{\text {trials }}\right)$ do

$n_{\text {correct }} \leftarrow 0$;

$\mathbf{T}_{\text {local }}^{r} \leftarrow$ calculate local pose of random point within $F_{\text {obs }}$;

for all elements within $p_{\text {subset }}$ do

$$
\epsilon^{i} \leftarrow\left\|\mathbf{T}_{\text {local }}^{r} \cdot \mathbf{p}_{\text {fts }}^{i}-\mathbf{p}_{\text {obs }}^{i}\right\|^{2} ;
$$$$
\text { if } \epsilon^{i}<\tau_{\text {error }} \text { then }
$$$$
\mid n_{\text {correct }} \leftarrow n_{\text {correct }}+1 \text {; }
$$

end

end

if $n_{\text {correst }}>n_{\text {subset }} . p_{\text {found }}$ then

found $\leftarrow$ true;

$\mathbf{T}_{o b j} \leftarrow \mathbf{T}_{l o c a l}^{r} ;$

else

$n_{\text {trials }} \leftarrow n_{\text {trials }}+1$;

if $\operatorname{minError}>\sum \epsilon^{i}$ then

minError $\leftarrow \sum \epsilon^{i}$;

$\mathbf{T}_{\text {min }} \leftarrow \mathbf{T}_{\text {local }}^{r}$;

end

end

end

if found $\neq$ true then

$\mathbf{T}_{o b j} \leftarrow \mathbf{T}_{\min } ;$

end

return $\mathbf{T}_{o b j}$; 


\subsubsection{Deformable Objects}

A key for deformable object pose estimation in this work is the assumption is the $A_{o 2}$, considered at section 4.1. Considering $A_{o 2}$, although the object is deformable, it is possible consider that its feature point is locally rigid and planar. This assumption lead us to the point that we can find the local poses by each local pose.

Another simplification that we consider here, is that we will not fully estimate the object pose. Instead, we will only obtain a rough pose estimation for AR registering. For this, we will only find some best transformation within the local transformations in order to render some object over the deformable object surface.

With the assumption and simplification adopted above, the deformable object pose can be estimated in a simple way just trying to find the local pose that best represents the deformed pose in which we desire to render the object. For this purpose, we first need to know the position $\mathbf{p}_{\text {render }}$ relative to the object surface space that the user wants to render some object.

Then, we try to find the nearest feature point and local pose to $\mathbf{p}_{\text {render }}$ by calculating

$$
\mathbf{T}_{\text {obj }}=\underset{\mathbf{T}_{\text {local }}^{i}}{\operatorname{argmin}}\left(\left\|\mathbf{p}_{\text {render }}-\mathbf{p}_{\text {fts }}^{i}\right\|\right)
$$

where $\mathbf{p}_{f t s}^{i}$ is the original position of the feature point. This is a simple and rough way to estimate the deformable object pose. However, it already can provide a pose for the AR registration.

\subsection{Chapter Conclusions}

In chapter we have presented our method to locally and globally estimate feature points poses based on RGB-D information. In order to achieve this pose, we separated the process in three parts. In the first part (section 7.1), we pre-process the feature points due to obtain spacial informations such as position $\mathbf{p}_{f t s}$, normal $\vec{n}_{f t s}$ and orientation, $\vec{o}_{f t s}$.

After this, at the second part processes (section 7.2), the observed feature points in the new frame in order to calculate the feature points local poses $\mathbf{T}_{\text {local }}^{i}$. Finally, we estimate the global object pose of different class of object based on those local poses (section 7.3).

Results obtained by our method using RGB-D cameras can be found at section 9.2 at chapter 9. However, in the next chapter 8 we will present our other local pose estimation method, this time, based on RGB information, information returned by conventional cameras. 


\section{RegressAR: Decision Forest Local Pose Re- gression based on RGB Information}

Figure 26 - Information flow of our RegressAR pose estimation approach based on RGB information.

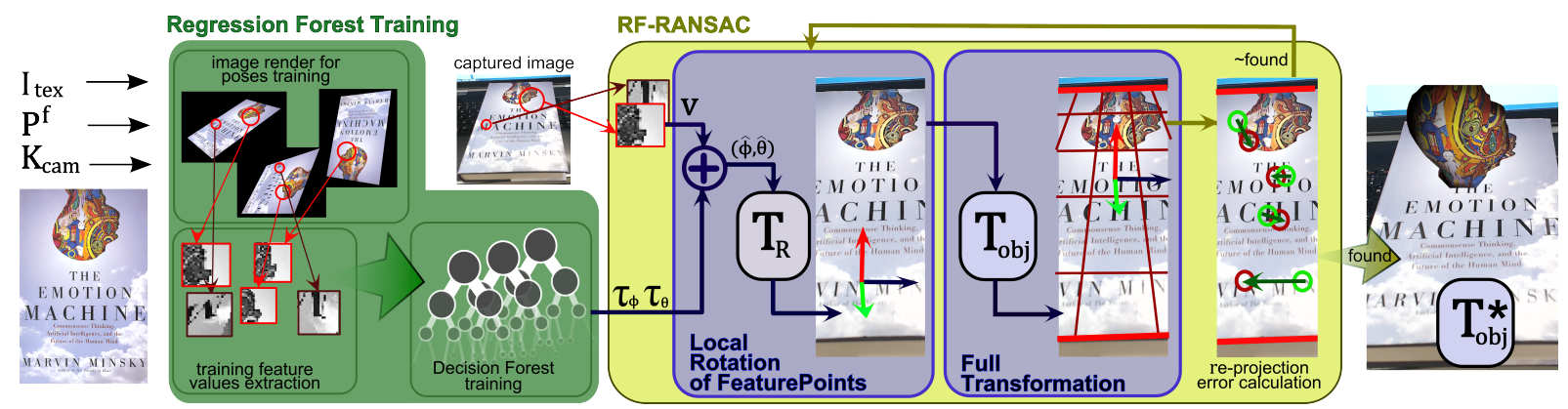

Source: Author.

In this chapter we present our approach for estimate the pose based only on RGB information, named RegressAR. The over all information flow of this approach is presented in figure 26. Here, in order to estimate the object pose, we apply a machine learning based approach, unlike conventional works, which apply the solve P- $n-\mathrm{P}$ approach. Some works in literature also use the machine learning for pose estimation. However, due to similarities of image patches across different views, the extension of those works is not trivial, as illustrated in figure 27.

In order to avoid such appearance similarities, we apply the machine learning process in rotation invariant features points. Our camera model are illustrated in figure 28. Note here that, once these feature points are rotation invariant, they are $\psi$ invariant. Thus, we can train the appearance changes in only 2 DoF instead of the full 3 DoF rotation, avoiding the similarities of appearances across views.

Our entire RegressAR approach is separated in four parts:

1. Regression forest training: which generates the regression forest from samples of the object feature in several poses (section 8.1);

2. local rotation pose estimation: which estimates the rotation pose of the feature point based on the observed feature point local information (section 8.2);

3. full transformation matrix estimation: estimates the translation value of the feature points by using a second point (section 8.3); 
Figure 27 - L2 distances between feature points views with fixed $\theta$, and varying $\phi, \psi$ values of camera. Zero valued distance in the graph is the reference view. It is possible to observe the similarity, which appears all across different $\psi$ and $\phi$, making the extension of $2 \mathrm{DoF}$ rotation regression (fixed $\psi$ ) to 3 DoF rotation non-trivial.
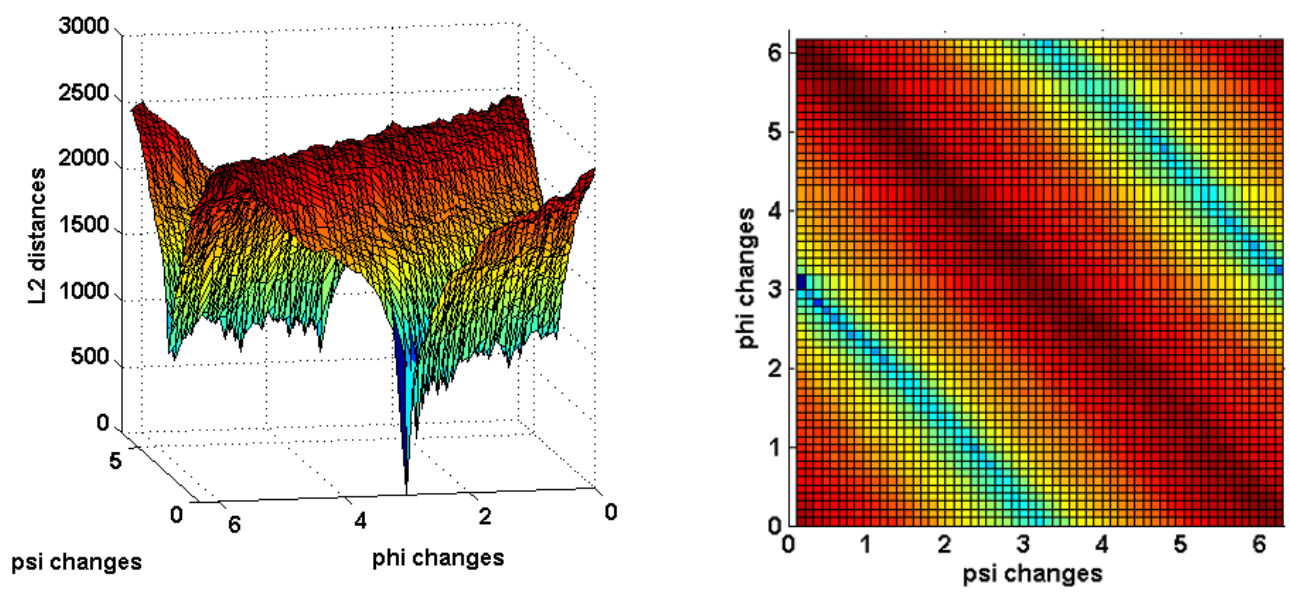

Source: Author.

Figure 28 - Camera pose models for regression. Camera model in figure 28a is used in the training part, and figure $28 \mathrm{~b}$, for the pose estimation part.

(a) Object centred coordinates
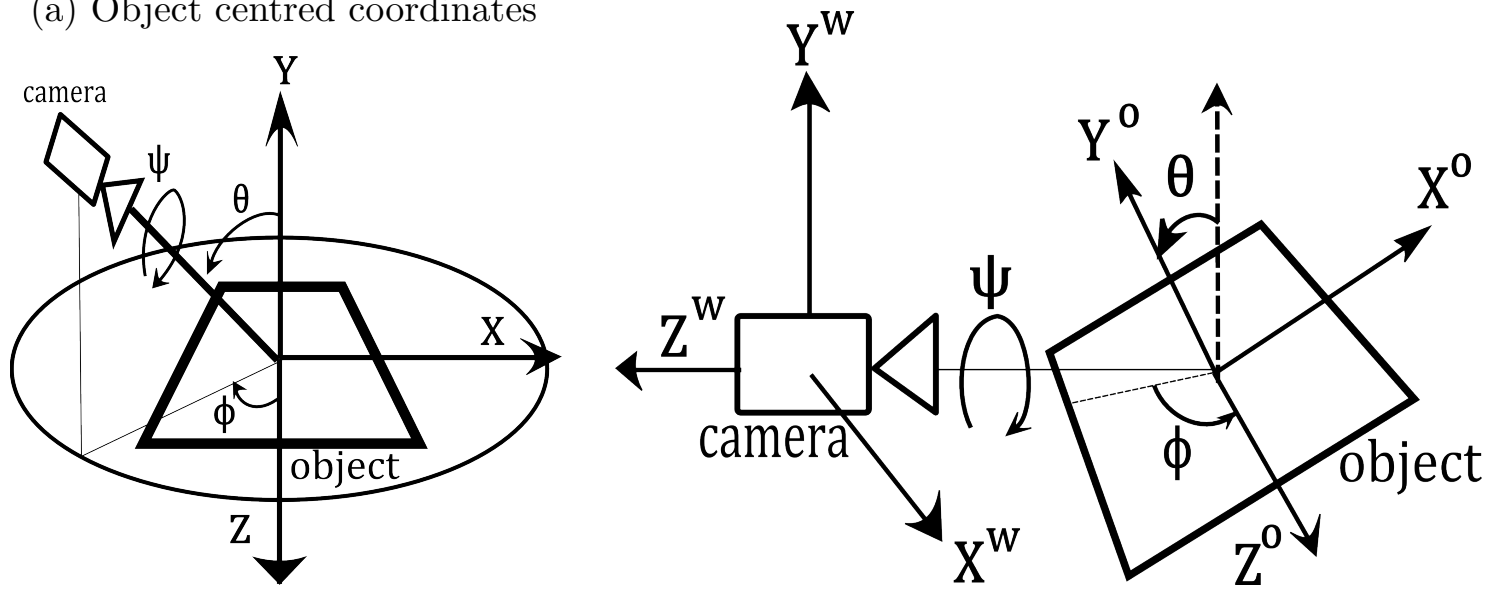

Source: Author.

4. Random Forest Random Sample Consensus (RF-RANSAC): estimates the global object pose by combining decision forest and RANSAC approaches (section 8.4).

\subsection{Regression Forest Pose Training}

The first part of our approach is the training of regression forest based on image patched of the feature points across different poses. Initially, several images of the object 
in different poses and positions are synthesized for the training, as presented in subsection 8.1.1. For each image, the training feature values across views are extracted from the feature points, subsection 8.1.2. Finally, those values are used as input for the decision forest training itself, as presented in subsection 8.1.3.

\subsubsection{Training Data Generation}

First, we use a synthetic approach to generate samples for training. Instead of capturing the real data object pose in several conditions, we render the object surface texture $\mathbf{I}_{t e x}$ as planar object with different camera poses, using the same camera projection matrix $\mathbf{K}_{\text {color }}$ of the capturing camera, as in chapter ??. This synthetic approach has the advantage to enable easy generation of a large of data set of ground truth data, as pointed by Shotton (CRIMINISI; SHOTTON, 2013).

We generate several examples of the object in different poses to extract the training feature values. In order to achieve the well spread representative sampling data for the training, we first generate samples with different $\theta$ values within $[\pi / 2, \ldots, 0]$ and $\phi$, within $[0, \ldots, 2 \pi]$, based on 28 a camera model. This camera model and poses are similar to our feature point extraction samples (chapters 6), however we generate a denser image set sample for this training.

Also, in order to train small errors created by rotation and scale values, we also need to train with random poses with different $\psi$ and $z$ values. Furthermore, we add a gaussian noise of mean 0 and several standard deviations $\sigma \mathrm{s}$, in order to make our regression results more robust to image noises. Here, we apply a camera models as in 28b, using several random values of $\theta, \phi, \psi, x, y$ and $z$ values.

\subsubsection{Training Feature Values Extraction}

In each new image, we first convert the color pixel values to brightness values and process a conventional feature matching algorithm (BAY et al., 2008) in order to find the matching feature points in the new image. Then, for each matched features point, we extract the feature point training feature values $\mathbf{v}_{t r}$.

This training features extraction is done by first extracting a small square image patch around the feature points. This image patch has a length of $l_{\text {diag }}$, which is based on the scale factor of the feature point, $s_{f t s}$. Therefore, our transformation in the image space is extracted with size $l_{\text {diag }}=n_{\text {pix }} s_{f t s} \sqrt{2}$. Where, $n_{\text {pix }}$ is the number of the pix related to the scale factor. Once, feature points in SURF and SIFT are blob like features, changing $n_{\text {pix }}$ greater then the conventional value used to analyse the interest point, makes the $\theta$ and $\phi$ regression better, although, it can make the patch capture also the background.

Then, the image patch is rotated using the inverse of orientation value $o_{f t s}$ of the 
feature point. This creates a image patch that always is oriented to the major orientation of the feature point, despite the $\psi$ value of the frame. Then, this new patch is cropped to fit $l_{\text {feat }}=n_{\text {pix }} s_{f t s}$, and re-scaled to the size of the training samples feature $l_{f t s}$.

This image patch is turned to a array of size $l_{f t s}^{2}$, in a raster scan manner, in order to obtain training feature values $\mathbf{v}_{t r} \in \mathbb{R}^{l_{f t}^{2}}$. At last, the feature values are normalized such as the minimum value is set to be 0 and maximum, to 1 , in order to make the feature less affected by brightness changes. Figure 29 illustrates this entire process.

This process is similar to applying a $2 \mathrm{D}$ transformation

$$
\begin{aligned}
\mathbf{T}^{I} & =\mathbf{T}_{t}^{I} \cdot \mathbf{T}_{\text {rot }}^{I} \cdot \mathbf{T}_{s}^{I} \\
\mathbf{T}_{t}^{I}= & {\left[\begin{array}{lll}
1 & 0 & p_{x}^{I} \\
0 & 1 & p_{y}^{I} \\
0 & 0 & 1
\end{array}\right], } \\
\mathbf{T}_{\text {rot }}^{I}= & {\left[\begin{array}{ccc}
\cos \left(o_{f t s}\right) & -\sin \left(o_{f t s}\right) & 0 \\
\sin \left(o_{f t s}\right) & \cos \left(o_{f t s}\right) & 0 \\
0 & 0 & 1
\end{array}\right], } \\
\mathbf{T}_{s}^{I}= & {\left[\begin{array}{ccc}
s_{f t s} \cdot n_{\text {pix }} & 0 & 0 \\
0 & s_{f t s} \cdot n_{\text {pix }} & 0 \\
0 & 0 & 1
\end{array}\right], }
\end{aligned}
$$

to the feature values grid. Where, $\mathbf{T}_{t}^{I}$ is a $2 \mathrm{D}$ translation matrix to the position $p_{x}^{I}$ and $p_{y}^{I}$ of the feature point; $\mathbf{T}_{r}^{I}$ is a $2 \mathrm{D}$ rotation matrix of angle $o$, the major orientation returned by the feature point; and finally, $\mathbf{T}_{s}^{I}$, a scale matrix of scaling factor $\left(s_{f t} n_{p i x}\right)$. Capturing the pixels within $\left[\left(-s_{t r} / 2,-s_{t r} / 2\right), \ldots,\left(s_{t r} / 2,-s_{t r} / 2\right), \ldots,\left(s_{t r} / 2, s_{t r} / 2\right)\right]$ of the grid, will return the same samples training features values. Figure 29 also illustrates this process. Graph 30 illustrates the changes of the first 15 values of the patch across changes in $\theta$ and $\phi$.

\subsubsection{Regression Forest Training}

For the training itself, we apply a regression forest approach. This choice is made, due to the capability of decision forests to generate fast regression results for real-time applications, among other advantages that decision forest approach has, such as presented by (CRIMINISI; SHOTTON, 2013). The decision forest approach is based on the multiple decision tree train, where each tree as returns a regression based on random features values and random data sample (CRIMINISI; SHOTTON, 2013). Therefore, we generate $n_{\tau}$ decision trees for each extracted feature point $f$.

Also, once we assumed that $\theta$ and $\phi$ are independent, we generate a set of $n_{\tau}$ trees 

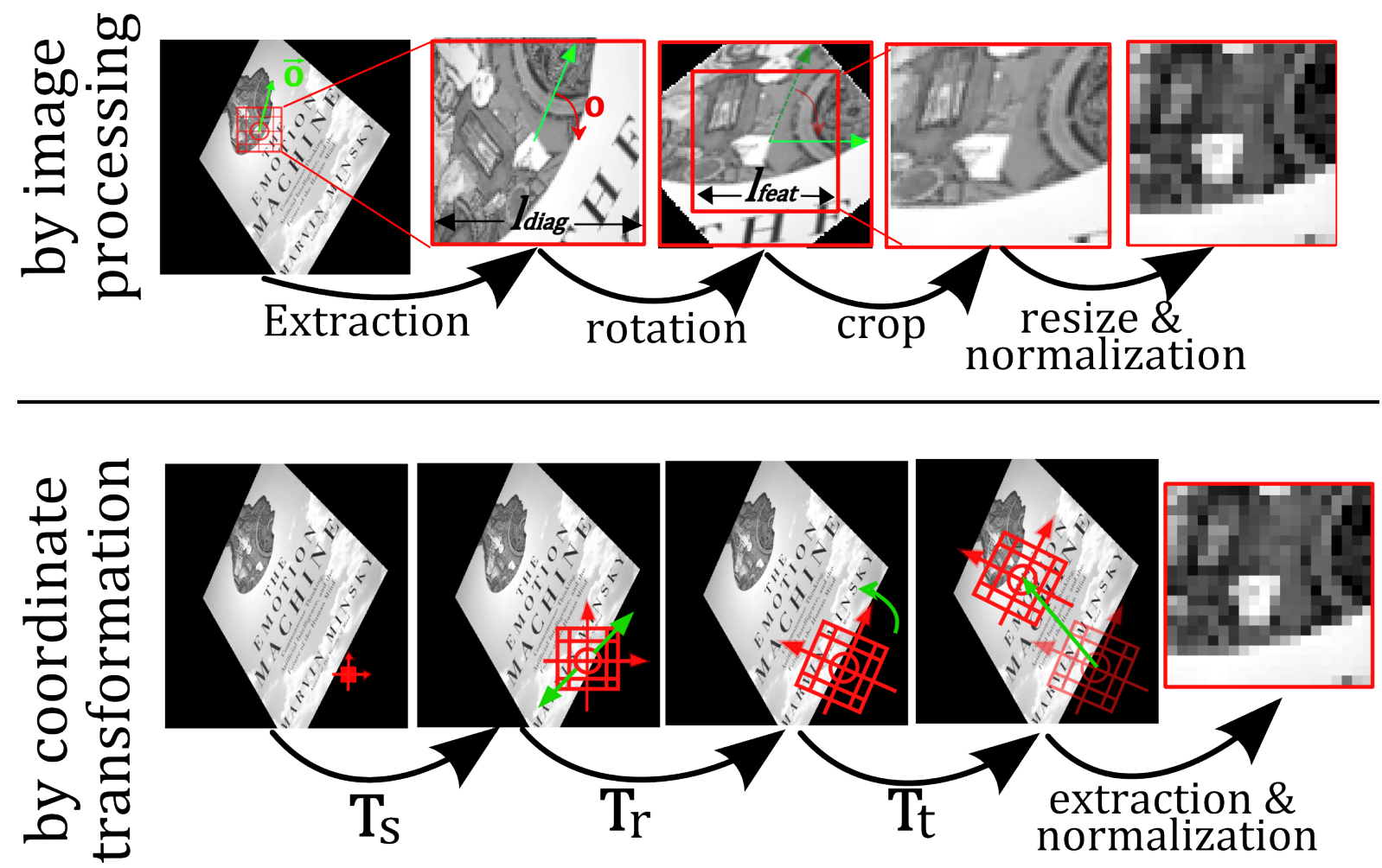

Figure 29 - Near feature point image patch extraction for training feature value $\mathbf{v}$ extraction. First row: extraction by image processing; second row: extraction based on coordinate system transformation.

for the $\theta$ value, $\tau_{(\theta, f)}$, and for $\phi$ values, $\tau_{(\phi, f)}$. However, decision forest also can be a memory expensive method, and training all the feature points of the object can rapidly increase the memory consumption. In order to avoid this problem, we can train only a set of $P^{r f}$ perspective robust feature point within $P^{f}$. Here, we select $P^{r f}$ as the $n_{r f}$ feature points that most appear across different rendered images.

Other feature points within $P^{f}$, are still used as verification points at the calculation process, as described later. At the end of this point, we have generated several feature points $P^{r f}$, each, attached with several regression trees $\tau_{(\theta, f)}, \tau_{(\phi, f)}$, where $P^{r f} \in P^{f}$, based the samples images.

\subsection{Local Rotation Matrix Estimation}

After obtaining the regression forest of the several feature points, for each new image frame capture $I_{o b s}$, we first proceed a feature matching algorithm to find the matching feature points between $p_{o b s}^{I}$ and $P_{f}, \mathbf{P}_{m}^{I}$. For each matched point $f$ within $P_{r f}$, points that has a regression forest associated to it, we first capture the image patch in the new image patch as in section 8.1.2.

We then, extract the feature values vector $\mathbf{v}_{f}^{t}$, of each matched feature point attached with regression forest, for the regression from the image patch. This feature 

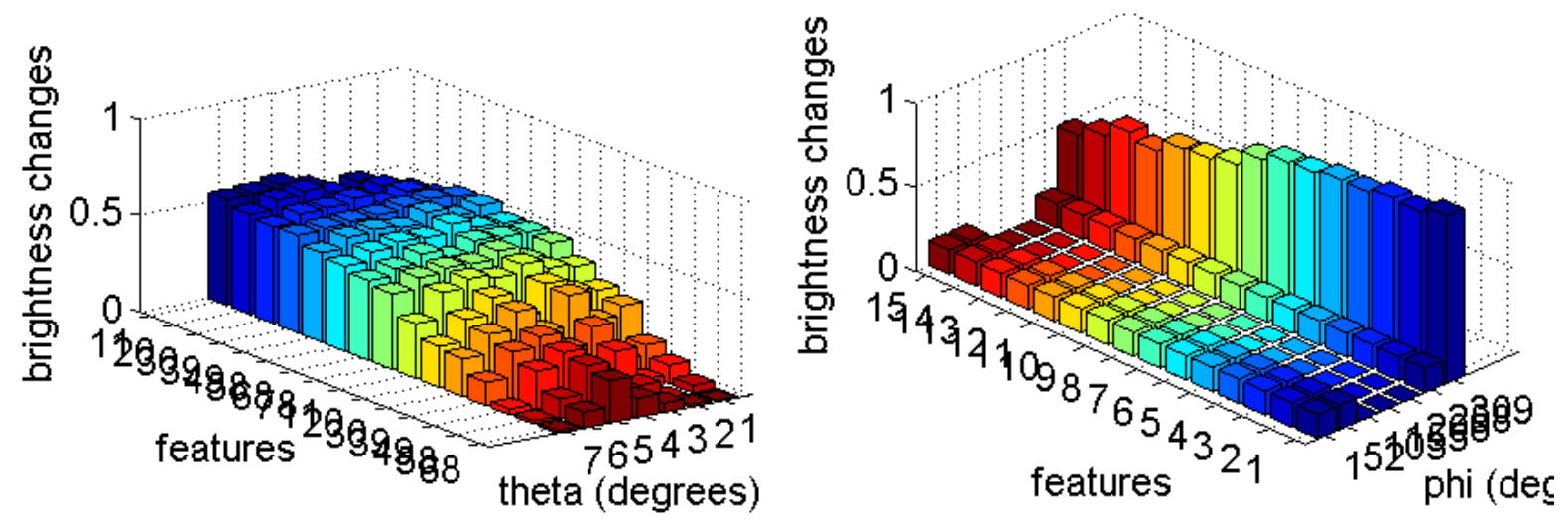

(a) feature values changes across $\theta$ changes with(b) feature values changes across $\phi$ changes with fixed $\phi$. fixed $\theta$.

Figure 30 - Training features values changes across $\theta$ and $\phi$ values, each one with different feature points. Illustrating the changes of pixel color across poses. Moreover, the difference between different feature points.

vector is then processed in each tree inside regression forest. Each regression tree will return a prediction of $\hat{\theta}$ and $\hat{\phi}$. Here, we first calculate the rotation matrix of the object based on the returned values of $\hat{\theta}$ and $\hat{\phi}$. This rotation transformation is calculated as

$$
\begin{aligned}
\mathbf{T}_{r} & =\mathbf{T}_{\psi} \cdot \mathbf{T}_{\phi} \cdot \mathbf{T}_{\theta} \\
\mathbf{T}_{\psi} & =\left[\begin{array}{cccc}
\cos (-\psi) & -\sin (-\psi) & 0 & 0 \\
\sin (-\psi) & \cos (-\psi) & 0 & 0 \\
0 & 0 & 1 & 0 \\
0 & 0 & 0 & 1
\end{array}\right], \\
\mathbf{T}_{\phi} & =\left[\begin{array}{cccc}
\cos (\phi) & 0 & -\sin (\phi) & 0 \\
0 & 1 & 0 & 0 \\
\sin (\phi) & 0 & \cos (\phi) & 0 \\
0 & 0 & 0 & 1
\end{array}\right] \\
\mathbf{T}_{\theta} & =\left[\begin{array}{cccc}
1 & 0 & 0 & 0 \\
0 & \cos (\theta) & -\sin (\theta) & 0 \\
0 & \sin (\theta) & \cos (\theta) & 0 \\
0 & 0 & 0 & 1
\end{array}\right]
\end{aligned}
$$

where, $\mathbf{T}_{\theta}$ is a rotation around the $x$-axis with $\theta ; \mathbf{T}_{\phi}$, around $y$-axis with $\phi$ and finally, $\mathbf{T}_{\psi}$, a rotation around the direction of the camera, $(-z)$-axis. However, once the regression is $\psi$-invariant, now we need to re-estimate the $\psi$ value for this new frame. This can be done by using a second known vector $\vec{v}_{\psi}$. 
This $\vec{v}_{\psi}$ can be calculated by a second matched 2D point, or also by the extracted orientation $o$ of the feature point, as done in our RGB-D approach (chapter 7). Using the orientation of the feature point itself enable us to calculate the rotation matrix of the object by only information from the feature point. This characteristic enable the calculation of local pose each feature point, enabling other unique results, as discussed at section 9.3.3. When using the orientation of the feature point itself, we consider $\vec{v}_{\psi}^{o}=\vec{o}_{f}^{o}$ and $\vec{v}_{\psi}^{I}=\vec{o}_{f}^{I}$, where $\vec{o}_{f}^{I}$ is the observed vector calculated by orientation $o$ returned by the feature point, $\vec{o}_{f}^{I}=[\cos (o), \sin (o)]$.

However, small errors can occur at the feature point orientation. These errors can lead in global pose error of the object. On the other hand, using a second point as $\overrightarrow{\mathbf{v}}_{\psi}$ gives us more stable results. This is true, specially when the two points are spread over the object surface, once they are less affected by local errors. In this case we consider $\vec{v}_{\psi}^{o}=\mathbf{p}_{f}^{o}-\mathbf{p}_{2}^{o}$ and $\overrightarrow{\mathbf{v}}_{\psi}^{I}=p_{f}^{I}-p_{2}^{I}$, where $\mathbf{p}_{2}^{o}$ is the original 3D location of the feature point and $\mathbf{p}_{2}^{I}$, the observed location of the second point at the image space.

After calculating $\overrightarrow{\mathbf{v}}_{\psi}^{I}$ and $\overrightarrow{\mathbf{v}}_{\psi}^{o}$, we calculate the final rotation matrix by finding $\hat{\psi}$ that matches the captured $\overrightarrow{\mathbf{v}}_{\psi}^{I}$ with transformed $\overrightarrow{\mathbf{v}}_{\psi}^{o}$, as illustrated by figure 31 . Initially, $\overrightarrow{\mathbf{v}}^{w}(\hat{\psi})$ is calculated to be the final position of the transformed $\overrightarrow{\mathbf{v}}^{o}$ as

$$
\overrightarrow{\mathbf{v}}^{w}(\hat{\psi})=\mathbf{T}(\hat{\psi}) \mathbf{T}_{\hat{\phi}} \mathbf{T}_{\hat{\theta}} \cdot \overrightarrow{\mathbf{v}}_{\psi}^{o}
$$

Where, $\mathbf{T}_{\hat{\phi}}$ and $\mathbf{T}_{\hat{\theta}}$ are the transformation matrix calculated by returned $\hat{\theta}$ and $\hat{\phi}$, and $\mathbf{T}(\hat{\psi})$ calculated with the unknown variable $\hat{\psi}$.

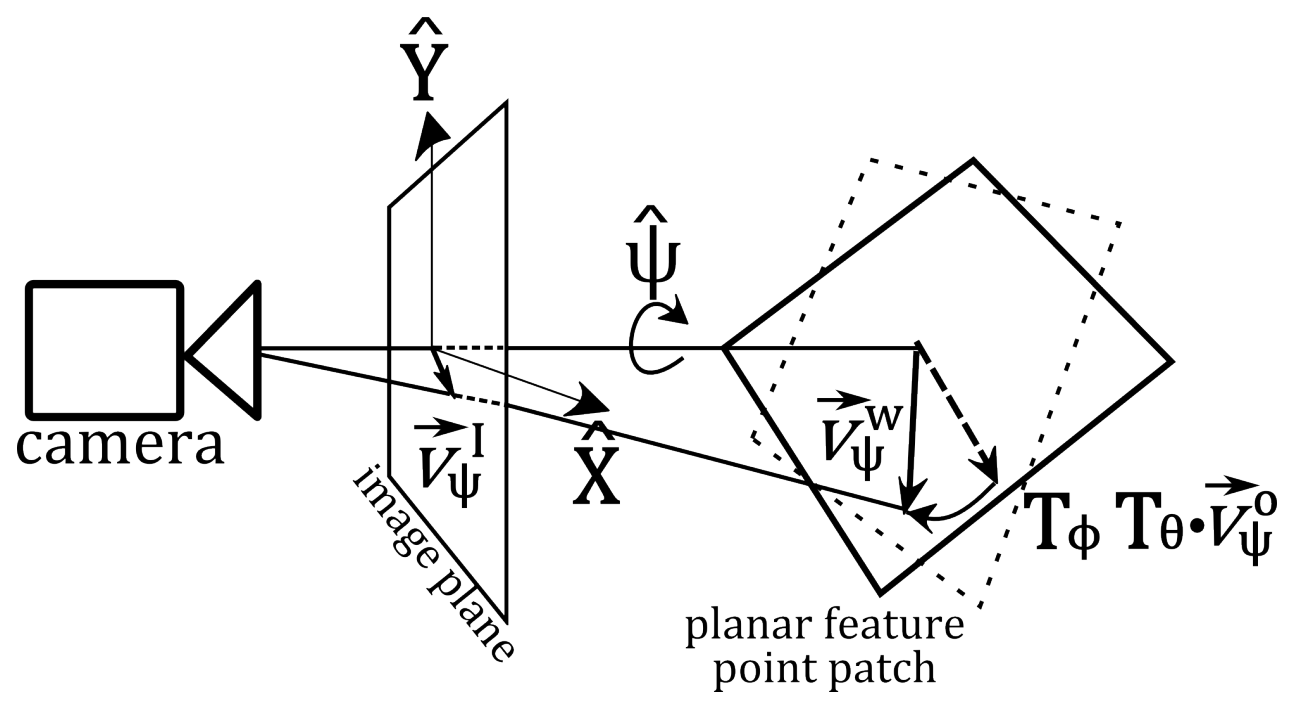

Figure $31-\psi$ calculation model.

Once $\overrightarrow{\mathbf{v}}^{w}(\hat{\psi})$ projected to the image plane, defined by $\hat{\mathbf{X}}$ and $\hat{\mathbf{Y}}$ axis, has same orientation as $\overrightarrow{\mathbf{v}}_{\psi}^{\text {obs }}$. Therefore, we have

$$
\left[\begin{array}{l}
\hat{\mathbf{X}} \cdot \overrightarrow{\mathbf{v}}^{w}(\hat{\psi}) \\
\hat{\mathbf{Y}} \cdot \overrightarrow{\mathbf{v}}^{w}(\hat{\psi})
\end{array}\right] \propto \vec{v}_{\psi}^{o b s} .
$$


Where, $\vec{v}_{\psi}^{o b s}$ is observed $\vec{v}_{\psi}^{I}$ projected to the world coordinates as

$$
\overrightarrow{\mathbf{v}}_{\psi}^{o b s}=\left(z_{i} \mathbf{K}_{c a m}^{-1} \mathbf{p}^{o}-z_{i} \mathbf{K}_{c a m}^{-1}\left(\mathbf{p}^{o}+\overrightarrow{\mathbf{v}}_{\psi}^{I}\right)\right) .
$$

Once, they must have same orientation, we can calculate $\tan (\hat{\psi})$ by

$$
\tan (\hat{\psi})=\frac{\overrightarrow{\mathbf{v}}_{\psi}^{o b s}[x]}{\overrightarrow{\mathbf{v}}_{\psi}^{o b s}[y]}=\frac{\hat{\mathbf{X}} \cdot \overrightarrow{\mathbf{v}}^{w}(\hat{\psi})}{\hat{\mathbf{Y}} \cdot \overrightarrow{\mathbf{v}}^{w}(\hat{\psi})}
$$

By calculating (8.6), we can find $\hat{\psi}$ that rotates the feature point to the same direction to $\vec{v}_{\psi}^{I}$. Therefore, being able to calculate the rotation matrix of the local feature point $\mathbf{T}_{r}$ by (8.2).

\subsection{Full Transformations Matrix Estimation}

After calculating the rotation matrix, we now estimate the full transformation matrix of each feature point. In order to estimate the 3 dimensional translation of the object, we need to estimate the $z$ position of the feature point. This is done by, again, applying the information of a second point $\mathbf{p}_{2}$. This calculation of $z$ is done by fitting the distance between two rotated point to the observed distance. This calculation of $z$ is done by fitting the distance between two rotated point to the observed distance. Figure 32 illustrates this calculation process.

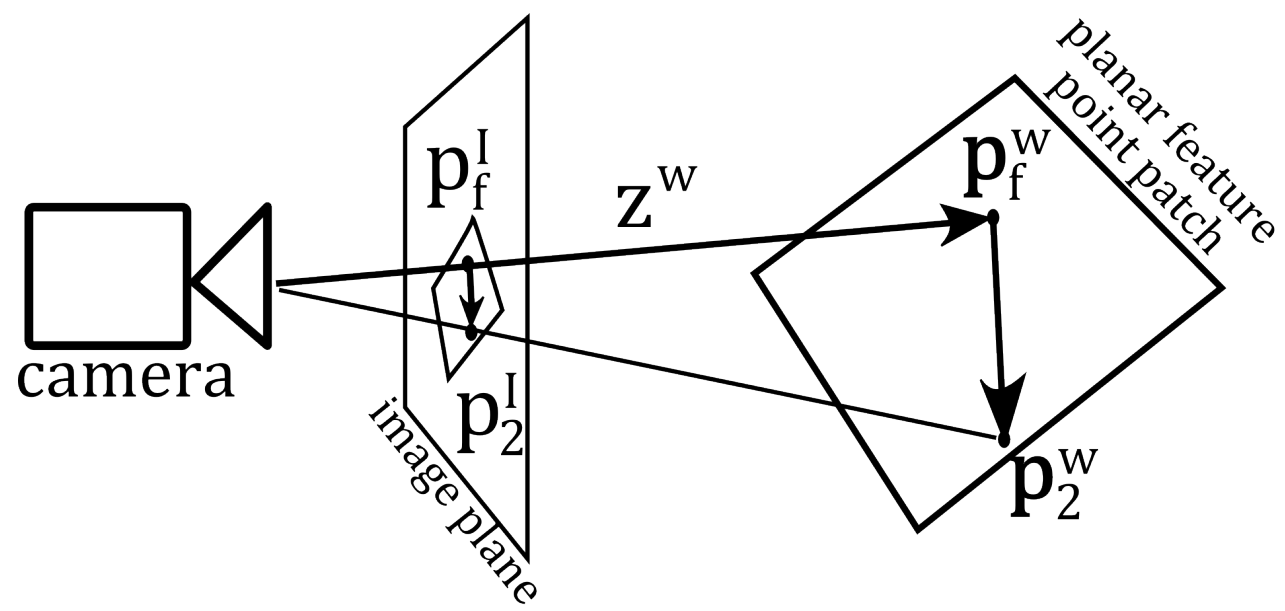

Figure $32-z$ calculation model in order to estimate the translation of the object pose.

First, we consider that the global position of a second point of the object $\mathbf{p}_{2}^{w}$, can be calculated by the sum of the position of the first observed feature point $\mathbf{p}_{f}^{w}$ and the rotated vector between both original positions $\mathbf{p}_{2}^{o}$ and $\mathbf{p}_{f}^{o}$. As

$$
\mathbf{p}_{2}^{w}=\mathbf{p}_{f}^{w}+\mathbf{T}_{r} \cdot\left(\mathbf{p}_{2}^{o}-\mathbf{p}_{f}^{o}\right)
$$


where, the global position of the observed feature point can be estimated by

$$
\begin{aligned}
\mathbf{K}_{c a m}\left(\mathbf{p}_{f}^{w}\right)^{H} & =p_{f}^{I} ; \\
\mathbf{p}_{f}^{w} & =z^{w} \mathbf{K}_{c a m}^{-1} p_{f}^{I} .
\end{aligned}
$$

Note that here, the only unknown variable is $z^{w}, z$ coordinate of $\mathbf{p}_{f}^{w}$. Also, note that this is true only for pair of points with rigid transformation, where the distance between both point does not changes across time.

Then, by projecting the second point to the image plane we have

$$
\begin{aligned}
\mathbf{p}_{2}^{I} & =\mathbf{K}_{c a m} \mathbf{p}_{2}^{w} \\
& =\mathbf{K}_{c a m}\left(z^{w} \mathbf{K}_{c a m}^{-1} \cdot p_{f}^{I}+\mathbf{T}_{r}\left(\mathbf{p}_{2}^{o}-\mathbf{p}_{f}^{o}\right)\right)^{H},
\end{aligned}
$$

where $p_{2}^{I}$ is the image coordinates of the observed second point. By (8.9), we can calculate the $z^{w}$ coordinate of the observed feature point global position. Finally object translation is given by $\mathbf{t}=z^{w} K_{\text {cam }}^{-1} p_{f}^{I}-\mathbf{T}_{r} \mathbf{p}_{f}^{o}$. By combining the rotation transformation obtained in section 8.2 and the translation matrix $\mathbf{T}_{t}$ calculated using $\mathbf{t}$, we obtain the object full transformation $\mathbf{T}_{o b j}$ by

$$
\mathbf{T}_{o b j}=\mathbf{T}_{t} \mathbf{T}_{r}
$$

As in our RGB-D approach (section 7.2), this results also needs to be rectified if the object is an rigid object using $f_{o b j}$ (section 4.1). Obtaining true object local transformation as

$$
\mathbf{T}_{f t s O b j}=f\left(\mathbf{T}_{o b j}\right) .
$$

\subsection{Global Pose of Rigid Objects: Random Forest Random Sample Consensus}

For rigid object, one simple way to estimate the final object pose, based on the regression returned by several trees of several feature points, could be done by only taking the mean of returned $\hat{\theta}$ and $\hat{\phi}$, as conventional approaches (CRIMINISI; SHOTTON, 2013), and using the same calculation of section 8.2 and 8.3. Despite this, several trees leads to wrong regression values, which will be illustrated in chapter 9.

This could happen because of similarities of the patch deformations in two different views. However, unlike conventional decision trees, where the regression value is calculated based on the input feature values $\mathbf{v}$ as

$$
p\left(\mathbf{T}_{o b j} \mid \mathbf{v}\right)=\frac{1}{Z} \prod_{i} p_{i}\left(\mathbf{T}_{o b j} \mid \mathbf{v}\right),
$$

here in this work, we have also other prior information about the object pose that could be used in order to refine the pose regression. 
One strong prior information, that we have, is the the distances between observed and re-projected $2 \mathrm{D}$ points of the feature points $\mathbf{p}_{m}^{I}=\left[\left(p_{0}^{I}, \mathbf{p}_{j}^{f}\right), \ldots,\left(p_{n}^{I}, \mathbf{p}_{k}^{f}\right)\right]$, such as used in many works of solve P- $n$-P problems (LEPETIT; MORENO-NOGUER; FUA, 2009; LI; XU; XIE, 2012). These information also can be applied to the forest regression in order to improve the overall accuracy.

We inject this prior information at (8.12) by calculating

$$
p\left(\mathbf{T}_{o b j} \mid \mathbf{v}, \mathbf{p}_{m}^{I}\right)=\frac{1}{Z} \prod_{i} p_{i}\left(\mathbf{T}_{o b j} \mid \mathbf{v}, \mathbf{p}_{m}^{I}\right) \text {. }
$$

By applying the Bayes rule (), (8.13) can be extended as

$$
\begin{aligned}
p\left(\mathbf{T}_{o b j} \mid \mathbf{v}, \mathbf{p}_{m}^{I}\right) & \propto \prod_{i} \frac{p_{i}\left(\mathbf{T}_{o b j} \mid \mathbf{v}\right) p_{i}\left(\mathbf{p}_{m}^{I} \mid \mathbf{T}_{o b j}, \mathbf{v}\right)}{p_{i}\left(\mathbf{p}_{m}^{I}\right)} \\
& \propto \prod_{i} p_{i}\left(\mathbf{T}_{o b j} \mid \mathbf{v}\right) p_{i}\left(\mathbf{p}_{m}^{I} \mid \mathbf{T}_{o b j}\right) .
\end{aligned}
$$

Here $p_{i}\left(\mathbf{p}_{m}^{I} \mid \mathbf{T}_{o b j}, \mathbf{v}\right)$ is the probability of finding the projected $\mathbf{p}^{o}$ to the image plane in $\mathbf{p}_{m}^{I}$ position by applying $\mathbf{T}_{o b j}$. Once the re-projected positions $\mathbf{p}_{m}^{I}$ are independent of the feature values $\mathbf{v}$ used in regression, $p_{i}\left(\mathbf{p}_{m}^{I} \mid \mathbf{T}_{o b j}, \mathbf{v}\right)$ can be simplified as $p_{i}\left(\mathbf{p}_{m}^{I} \mid \mathbf{T}_{o b j}\right)$. Also, once $p_{i}\left(\mathbf{p}_{m}^{I}\right)$ is equal for all trees, we can obtain the approximated probability without $p_{i}\left(\mathbf{p}_{m}^{I}\right)$.

This re-projection probability $p_{i}\left(\mathbf{p}_{m}^{I} \mid \mathbf{T}_{o b j}\right)$ is inversely related to the sum of bidimensional distance errors $\epsilon^{I}$ between the re-projected point, transformed by $\mathbf{T}_{o b j}$, and observed point. This error can be calculated as

$$
\epsilon^{I}=\left\|p_{i}^{I}-\mathbf{K}_{c a m}\left(\mathbf{T}_{o b j} \mathbf{p}_{i}^{o}\right)^{H}\right\| .
$$

Hence, as the distance $\epsilon$ increases, the probability $p_{i}\left(\mathbf{p}_{m}^{I} \mid \mathbf{T}_{o b j}\right)$ of $T_{o b j}$ decreases; we can approximate $p_{i}\left(\mathbf{p}_{m}^{I} \mid \mathbf{T}_{o b j}\right)$ to be inversely proportional to $\epsilon^{I}$.

We now can re-write equation estimate the best transformation $T_{o b j}^{*}$ as

$$
\mathbf{T}_{o b j}^{*}=\arg \max p\left(\mathbf{T}(\theta, \phi) \mid \mathbf{v}, \mathbf{p}_{m}^{I}\right) .
$$

Once $T(\theta, \phi)$ is calculated by the $\hat{\theta}$ returned by the trees $\tau_{(\theta, f)}^{j}$, and $\hat{\phi}$, by $\tau_{(\phi, f)}^{k}$, both from feature point $f$, and, we consider that the probability of the returned trees are constant, we can develop (8.16) as

$$
\arg \max p\left(\mathbf{T}(\theta, \phi) \mid \mathbf{v}, P_{m}^{I}\right) \approx \underset{i, j, k}{\arg \max } p_{i}\left(P_{m}^{I} \mid \mathbf{T}\left(\theta_{(j, i)}, \phi_{(k, i)}\right)\right) .
$$

Finally, we have that $p_{i}\left(\mathbf{p}_{m}^{I} \mid T\left(\theta_{j}, \phi_{k}\right)\right)$ is inversely proportional to sum of the re-projection error $\epsilon$, as in (8.15), thus having

$$
\begin{aligned}
\mathbf{T}_{o b j}^{*} & \approx \underset{i, j, k}{\arg \min } \sum_{l} \epsilon_{i, j, k}^{I} \\
& \approx \underset{i, j, k}{\arg \min } \sum_{l}\left\|p_{m, l}^{I}-\mathbf{K}_{c a m}\left(\mathbf{T}\left(\theta_{(j, i)}, \phi_{(k, i)}\right) p_{l}^{o}\right)^{H}\right\| .
\end{aligned}
$$


By (8.18), we can calculate the final $\mathbf{T}_{o b j}^{*}$ as the transformation that has least re-projection error calculated by all combinations of $\hat{\theta}$ and $\hat{\phi}$ returned from all the feature points $f$.

However, calculating all the re-projections of the matched points, with all possible $\hat{\theta}, \hat{\phi}$ trees regression of all feature points $f$ can be computationally heavy. In order to make this process faster for real-time applications such as in AR, we also propose here a hybrid approach between our pose regression forest with RANSAC method (MOREL; YU, 2009), named as Random Forest Random Sample Consensus (RF-RANSAC).

In our RF-RANSAC approach, we combine the random sampling idea inside the decision forest. Thus, not only randomly sampling the observation nor features to be used, as a conventional approach, but also randomly sampling the trees within all the decision trees of all feature. This sampling gives us robustness to outlines, as in original RANSAC approach (MOREL; YU, 2009), and also more performance, once we do not need to regress all the trees in the decision forest, as in a conventional decision forest approach.

As in (MOREL; YU, 2009), we first sample a subset $S 1$ as a percentage $p_{s}$ of all matched feature points within $\mathbf{p}_{m}^{I}$ to use in the re-projection error calculation. Then, randomly, we choose a matched feature point $p_{i}^{f}$ that has a decision forest attached in. We then, calculate the possible transformation $T_{o b j}$ by extracting the regression feature values $\mathbf{v}_{i}$ of $p_{i}^{f}$ in the new image. Then, we, also, randomly choose one $\theta$ regression tree $\tau_{(\theta, i)}^{j}$, and a $\tau_{(\phi, i)}^{k}$ for $\phi$, within in $p_{i}^{f}$ decision forest, in order to obtain $\hat{\theta}$ and $\hat{\phi}$ by $\mathbf{v}$. After this, as in 8.3, we calculate the object transformation $\mathbf{T}_{o b j}$.

We calculate the re-projection error of the observed point and $\mathbf{T}_{o b j}$ similar to (8.15), using $S 1$. If the number of point that has $\epsilon^{I}$ smaller then distance threshold $d_{t h}$, is greater then acceptance percentage $p_{t h}$ of the subset, we accept $\mathbf{T}_{o b j}$ as the object pose, i.e, we calculate if

$$
\#\left[\epsilon^{I}<d_{t h}\right]>p_{t h} \cdot p_{s} \cdot N,
$$

where $N$ is the total of matched feature points. Elsewhere, we keep re-estimating $\mathbf{T}_{o b j}$ by using other random sample of feature point, $\theta$-tree and $\phi$-tree, while (8.19) is not satisfied. Finally If we not found a $\mathbf{T}_{o b j}$ that satisfies (8.19) within $n_{\text {trial }} \mathrm{s}$; we then, set as object transformation $\mathbf{T}_{o b j}$ as the transformation that had minimal error in the $n_{\text {trials }}$, as in (8.18). We illustrates the entire RF-RANSAC process at the algorithm 5.

\subsection{Chapter Conclusion}

In chapter we have presented our approach to locally and globally estimate the pose of feature points based on RGB information, named RegressAR. In order to obtain the object pose estimation, we applied a machine-learning based approach. By training the machine to learn the appearance changes of local images patches across several perspective change (section 8.1), we can obtain the rotation of the camera with 2 DoF (section 8.2). 


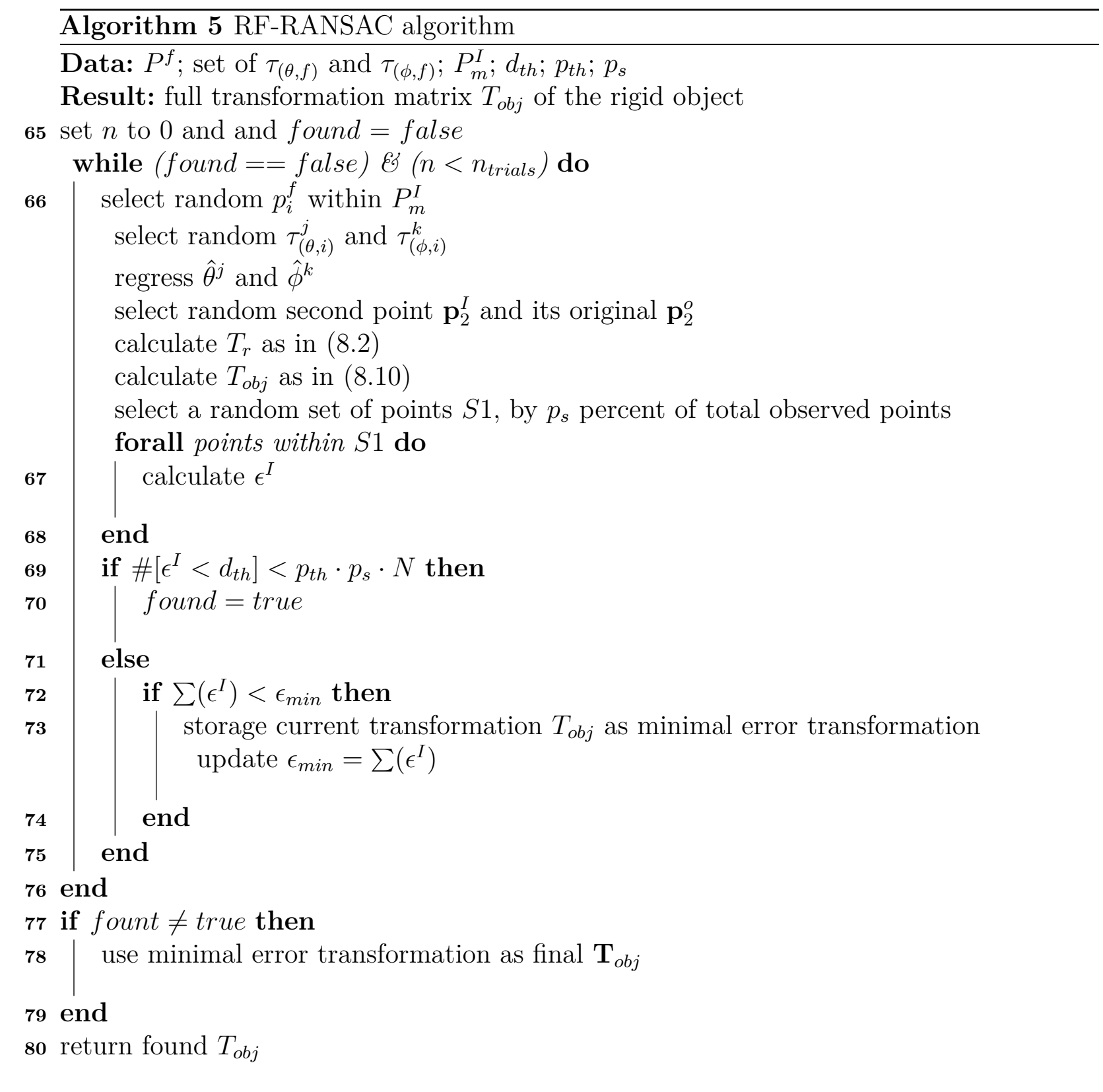

Then, we use the feature point orientation to find the third rotation (section 8.3). In order to refine the overall object pose, we combine the RANSAC method with the decision forest (section 8.4). By applying this method (named RF-RANSAC) we can find the object final pose.

In the next chapter (chapter 9), we present the results obtained by our approaches and methods applied to obtain the object poses in RGB and RGB-D, as well as, the robust feature point extraction. Results of our RegressAR approach can be found at 9.3. 


\section{Results}

In this chapter we present all the results obtained across this work. Our results are separated in 3 parts. The results obtained by our robust feature points extraction method, section 9.1; the pose estimation based on local poses using RGB-D cameras, 9.2; and, at last, using decision forest regression with RGB images 9.3. In section 9.4 we will discuss our results, and finally, we present our conclusion about our results in section 9.5.

\subsection{Robust Feature Point Extraction}

Our first results are related to the robust feature points extraction method proposed in this work. Initially image 33 shows the results of applying the background robust features extraction presented in 6.2. In this image, each green circle represents a found feature point, where the size of the circle represents the scale of the feature point.

Figure 33 - Results obtained by our background robust feature extraction method. At left, conventional SURF point extraction method applied to the image; at right, our method.

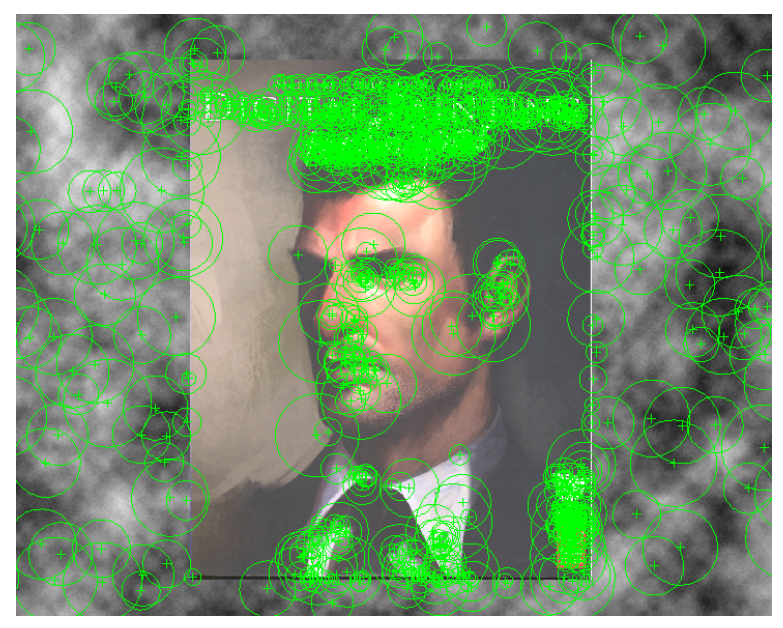

(a) conventional SURF

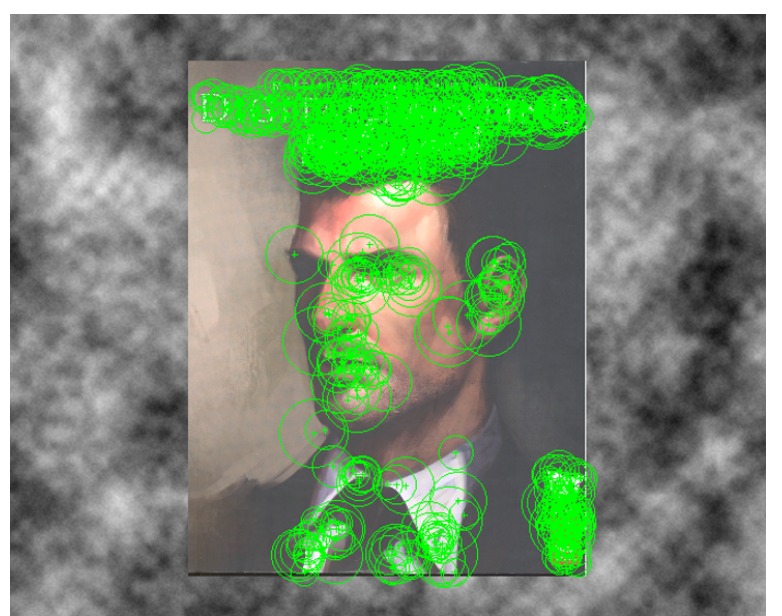

(b) Our background robust features

Source: Author.

From the results above, it is possible to observe that feature points that are found at the background are excluded, giving us only feature points inside the object. However, more importantly, we need to observe that the almost all the feature point region is also inside the object differently from the conventional methods, (BAY et al., 2008). This property is specially important for our local pose estimation methods, at chapters 7 and 8 , considering that the surrounding information are of the object surface texture. Without this property, the estimation of local poses can lead to wrong values. 
In figure 34 we show the results obtained with the feature point match in synthesized and real images, using our ASIFT modification, without 6.3 and with the entropy maximization method 6.4. Also it presents differences between the methods and plain SURF feature points extraction, as presented in the original work (MOREL; YU, 2009), extracting the feature points directly from the object texture image without any process. Here, we have listed exactly the same number of features of a conventional SURF feature extraction method to be able to compare the results, once our method can generate more number of feature points than the conventional method.

Figure 34 - Feature matching results using features extracted by different methods. At first row we show results using synthesized data, and second row, capture image from real environment. First column: feature extracted using conventional SURF methods; second column: our modified ASIFT; third column: our method of entropy maximization method.

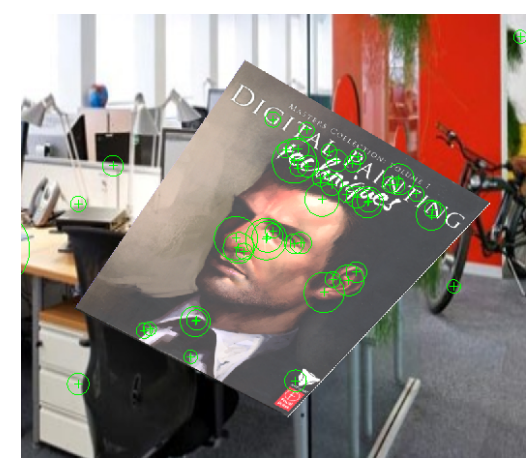

(a) conventional SURF

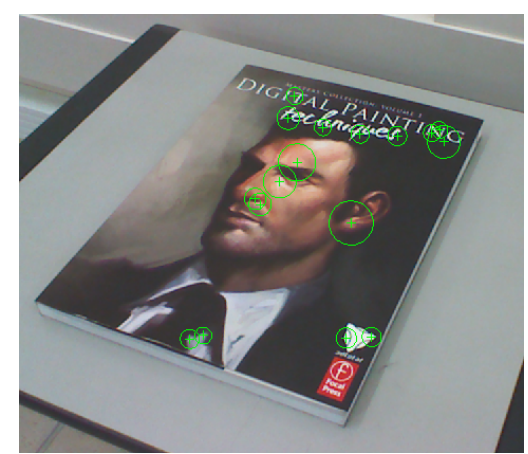

(d) conventional SURF

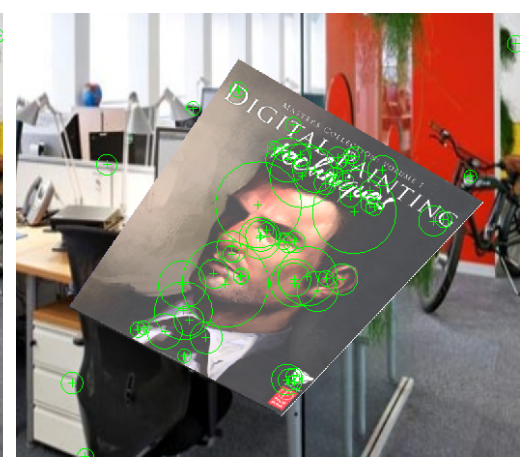

(b) ASIFT modification

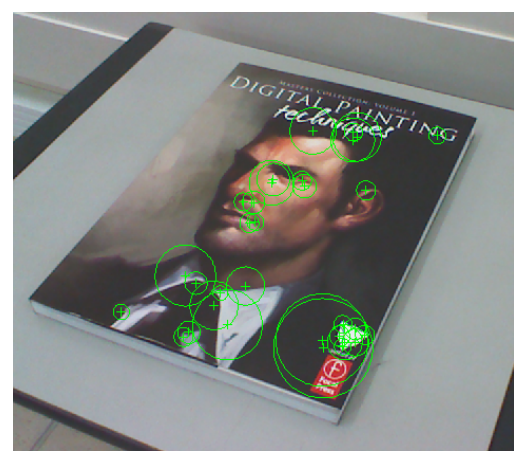

(e) ASIFT modification

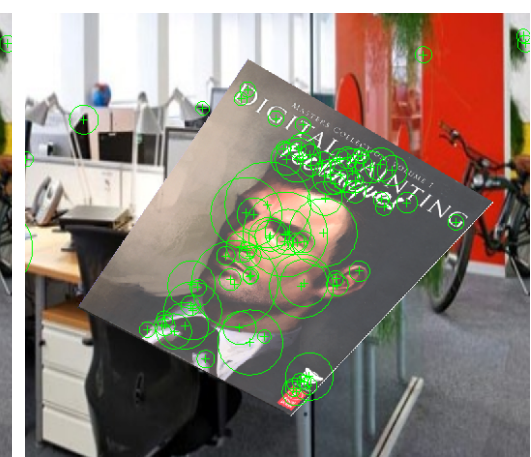

(c) Entropy Maximization

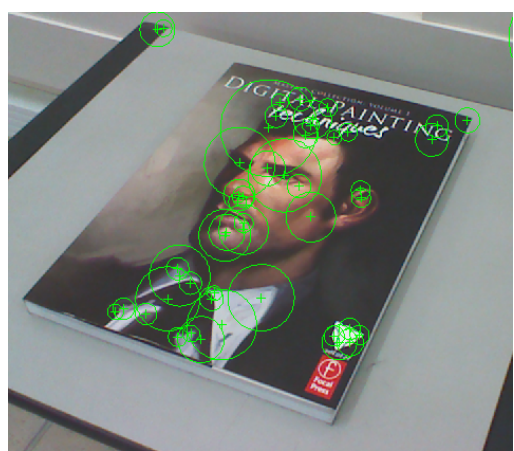

(f) Entropy Maximization

Source: Author.

From this result, it is possible to observe that our method has an increase in the number of match feature points, specially when the entropy maximization method is applied. Again, here we use the same number of feature points in all the 3 methods.

In figure 35, we present the box plot ${ }^{1}$ of matched feature points at synthesized

1 box plots are graphs which represents the median (the middle line in red), the IQR, which is in total the $50 \%$ of the probability density function (presented by the box), the 1.5IQR above and bellow (the extending lines) and the observation fall (in red crosses). Also, the mean values are represented as the middle of the boxes. 
views of the object. Here, we generated 671 images in different views with the camera placed in different latitudes and zeniths angle values, similarly to section 6.3. However, here, we render the object with constant latitudes, $\theta=\pi / 2-(\pi / 2)(t / 12)$ and $t=0, \ldots, 10$, and zeniths, $\phi=2 \pi(u / 60)$ where $u=0, \ldots, 60$.

Figure 35 - First row: feature matching results box plot across view latitude; second row: means plot in same graph.

(a)

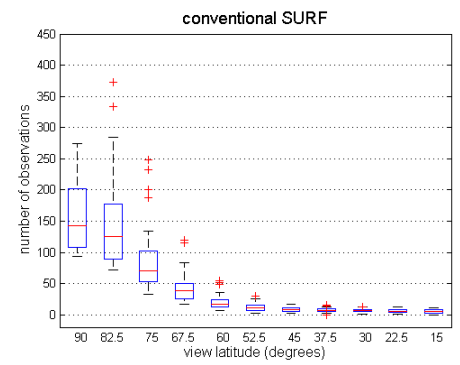

(b)

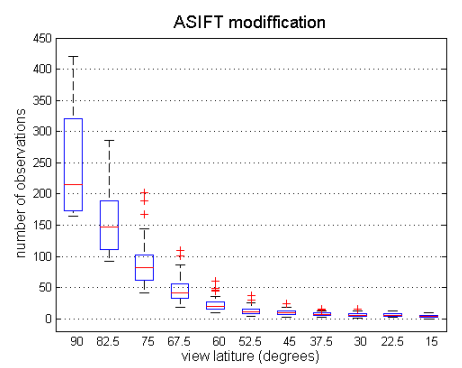

(c)

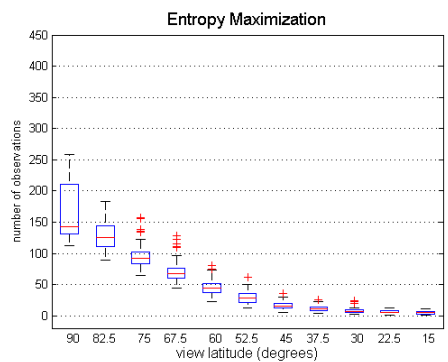

(d)

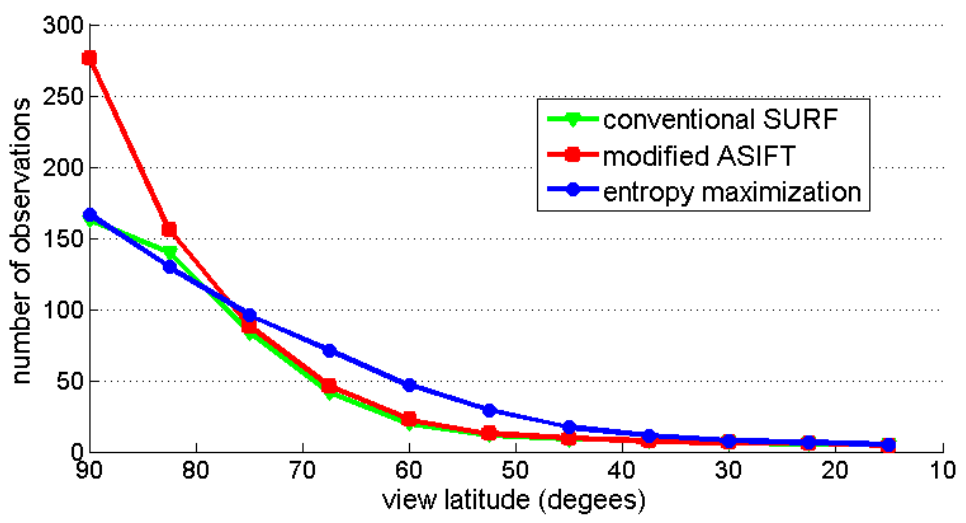

Source: Author.

From these images, it is possible to observe that our entropy maximization method has more spread observations of feature points across views, differently of the other two methods. Moreover, our method is almost always better, and when not, at least as good as the conventional SURF method. Also, from 35d it is possible to observe that picking only the feature points that has better number of views can lead to clustered feature points at top views, as shown by the curve of modified ASIFT method. It presents a peak at the orthogonal views, and quickly decreases as good as the conventional SURF features, proving our hypothesis presented at 6.4.

From these facts, it is possible to observe that our method can extract better feature points that will be robust thought different view conditions, when compared to the conventional method, even with the same number of features to match. Moreover, in these tests we have limited us with the same number of feature points as the original method 
result, but another great advantage in ASIFT methods is that we can extract more feature points from all views.

By figure 36, we present the mean observed points of the object rendered with random poses. Here, we vary the total number of feature points used in the matching algorithm with the new frame. We applied this matching using our entropy maximization method, the ASIF variant, and the conventional SURF detector again, in 150 images with the object in a random pose.

Figure 36 - First row: feature matching results box plot across number of extracted feature points use to match feature points at the new frame; second row: means with standard deviation plot in same graph.

(a)

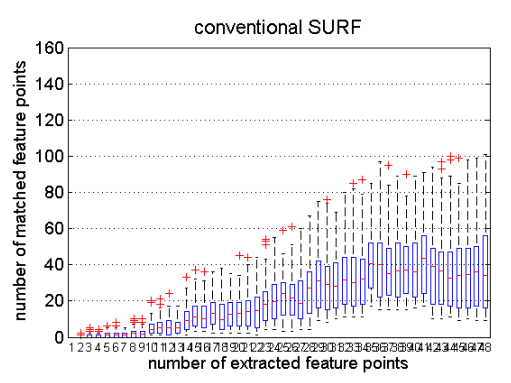

(b)

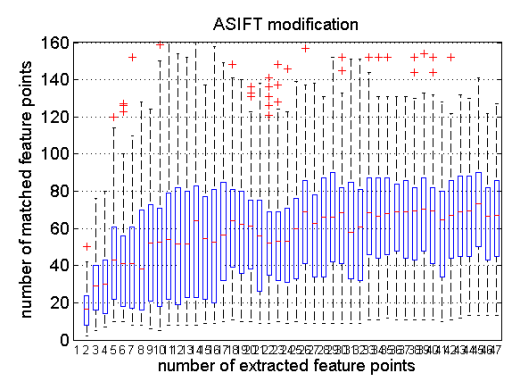

(c)

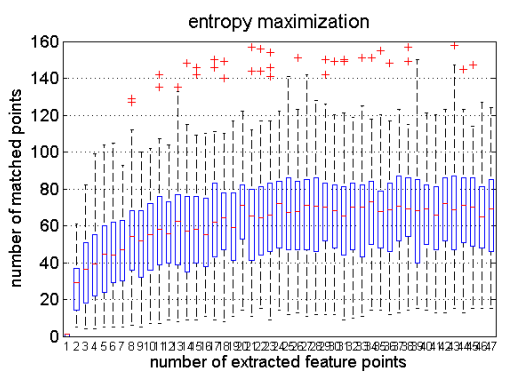

(d)

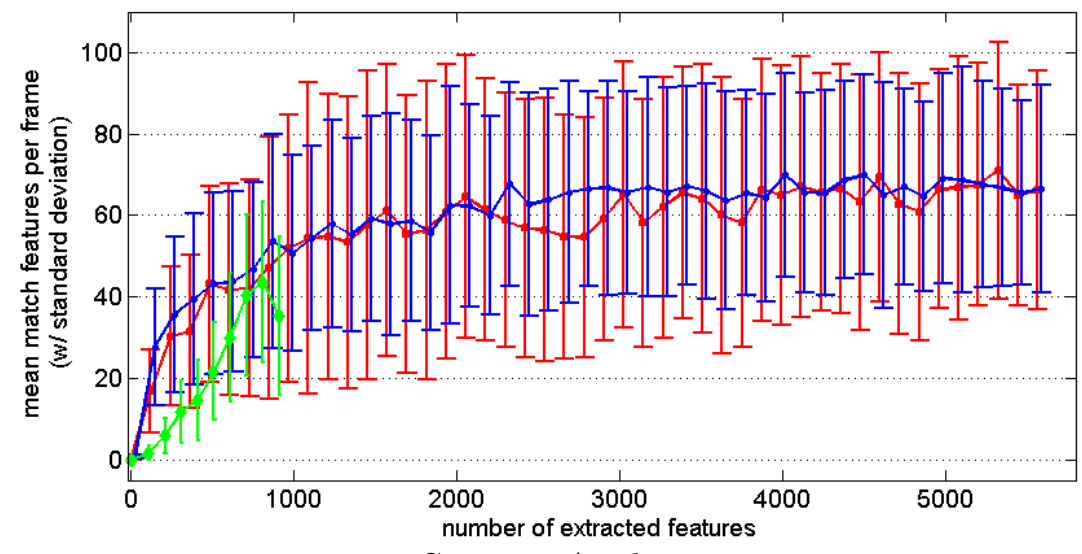

Source: Author.

One interesting characteristic is that our methods, of modified ASIFT and entropy maximization, has almost same visualization across views, and increase the visualization more quickly then conventional features, showing that we are picking good feature points from the beginning. Also, we can conclude that from certain number of features, the visualization turns to be stable, probably because of the total number of features at the new frame.

However, it is possible to see from 36b, 36c and 36d, that entropy maximization method has less variations across the increasing number of feature points, compared to the ASIFT method, once it has less standard deviation and IQR at the boxplot. Showing, 
again, that this method is more stable across views. Also, these figures show that entropy maximization method has faster increase of visualization then modified ASIFT with less feature points. This effect is shown with more detail at figure 37

Figure 37 - First row: feature matching results box plot across number of extracted feature points use to match feature points at the new frame with less numbers; second row: means with standard deviation
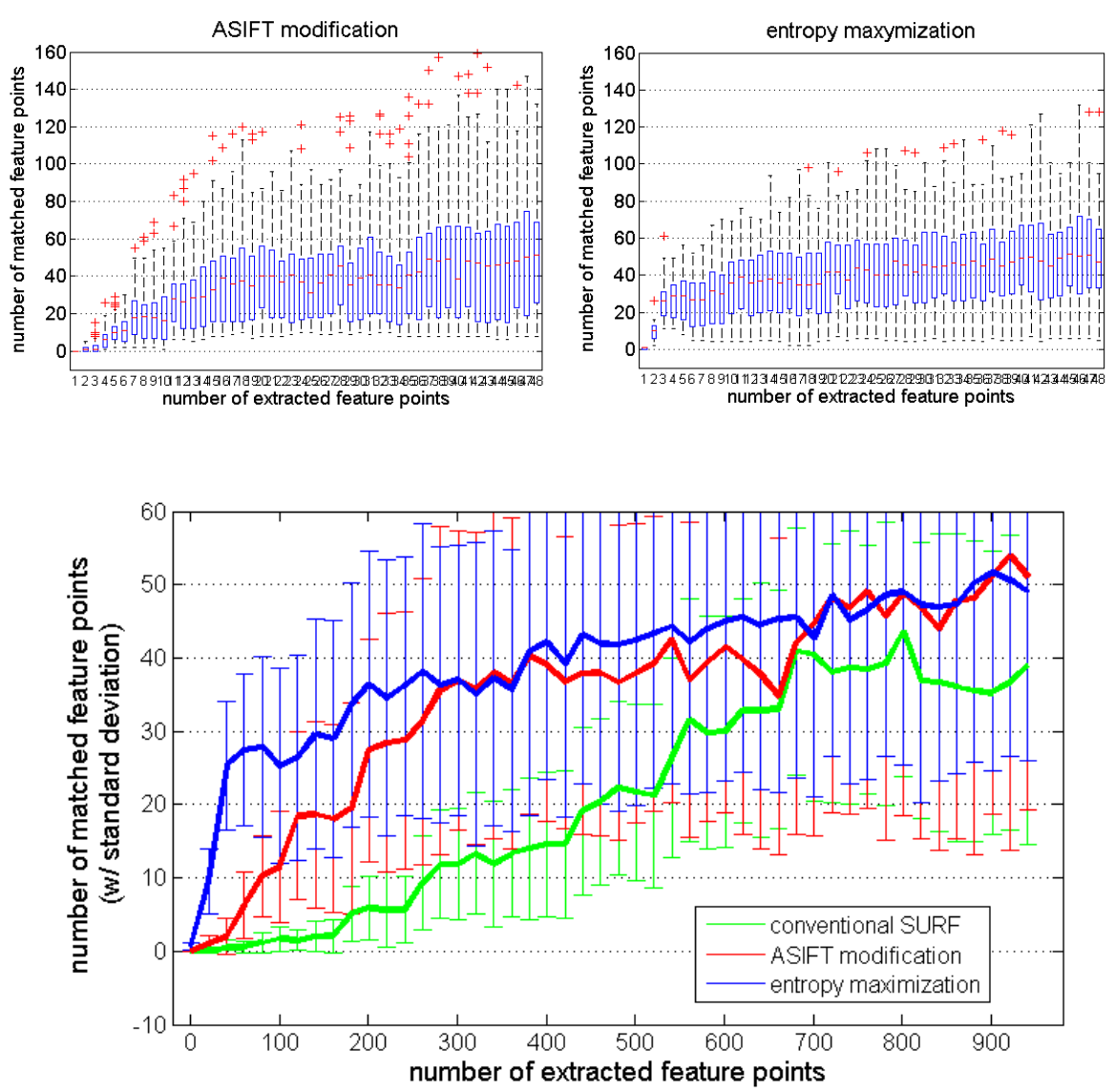

Source: Author.

In these figures, we show the relation of the extracted feature point number with matched number of features points in a new frame. Yet, with a smaller range of number of extracted features (varying across [1 to 952] feature points) this time, in order to be matched with the new scene. With these figures, it is more clear that the entropy maximization has better visual coverage across object poses than the modified ASIFT and conventional SURF methods when a smaller number of feature points are used for the point match algorithm. These results are important as pointed at section 6.5 once they directly affect the accuracy and precision of the estimation of the object global poses, shown in the following sections. 


\subsection{Pose Estimation by Local Pose Based on RGB-D Information}

After extracting the feature points, we process the local poses of it, and then, calculate the global pose of the objects. Here, in this section, we present the results obtained by applying our local pose estimation method using RGB-D information. As well as, the results of global estimation of poses based on the RGB-D local poses.

Figure 38 show our estimation results of planar object local poses, using RGB-D information using real data. In this image, each found pose is rendered with its coordinate system, where blue is the $\mathrm{X}$ axis, red, $\mathrm{Y}$ axis and green, $\mathrm{Z}$ axis. This coordinate system is rendered over the found feature point position.

Figure 38 - Local pose of planar object calculated with our RGB-D local pose method.
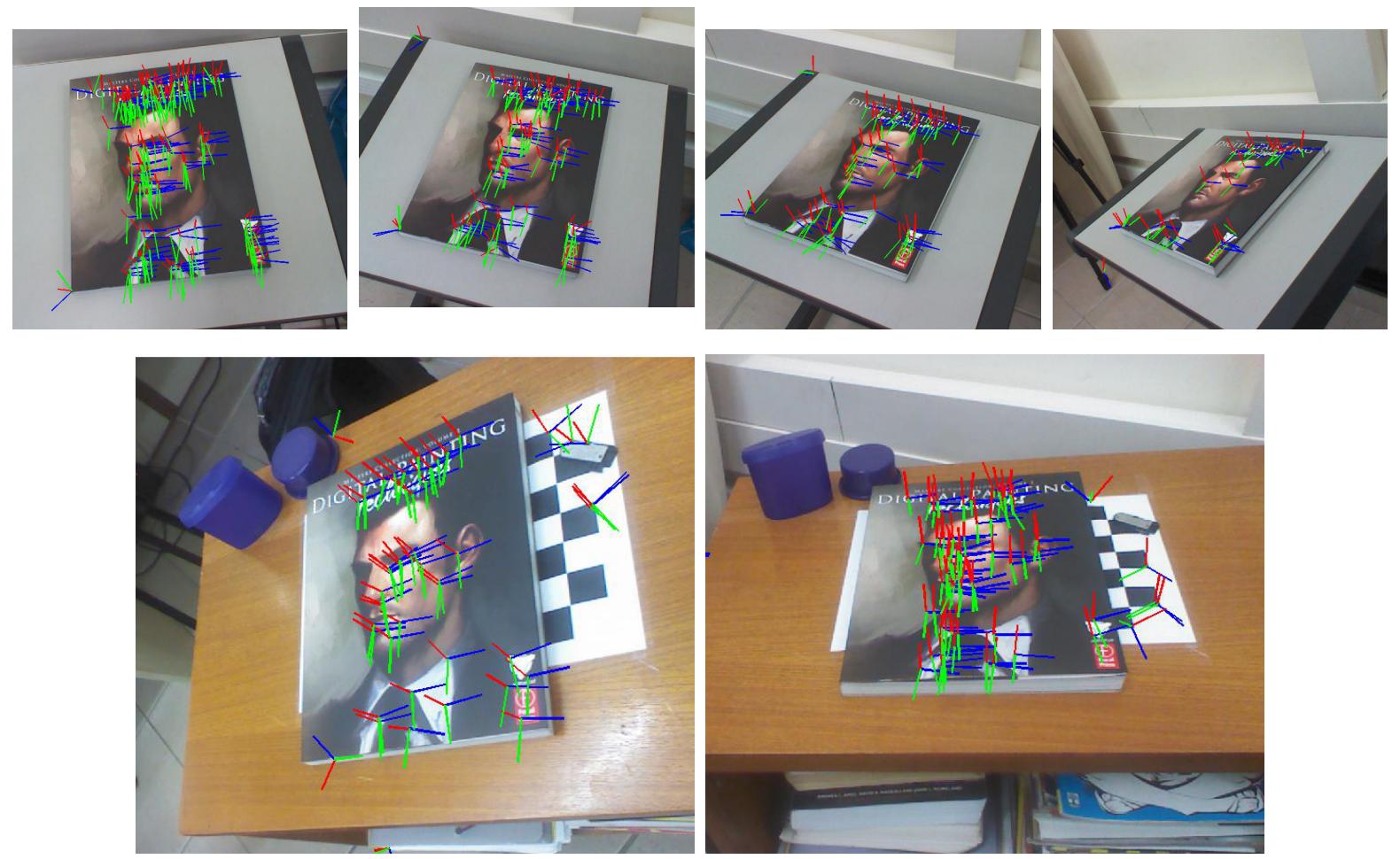

Source: Author.

From the images at 38 , it is possible to observe that the major number of feature points poses are, indeed, matching with the overall object pose. However, as addressed at 7.3 , there are a small error that happens across all the poses that are mainly in the orientation of $\mathrm{X}$ and $\mathrm{Z}$ axis. It is possible to observe that $\mathrm{Y}$ axis has not big oscillations across the surface, once is based on the normal information of the surface. But, there are small oscillations in X and Z. Such as presented at figure 39.

In the figure $39 \mathrm{~b}$ and $39 \mathrm{~d}$ we plot the angular error of the $\mathrm{X}$ and $\mathrm{Y}$ axis compared to the groundtruth object pose in a synthesized image, and the angular difference between the extracted global pose and all local poses of an planar object using real world data. Here, this angular error is calculated as $\alpha=\operatorname{acos}\left(X_{\text {local }} \cdot X_{\text {groundTruth }}\right)$. Where $X_{\text {local }}$ is the 
Figure 39 - Local pose detected X,Y-axis angles between ground truth (for synthesized image) and minimum observation error pose (for real image). First row: poses from synthesized image; second row, from real image. First column: the estimated pose image; second column: graph of the angles.

(a)

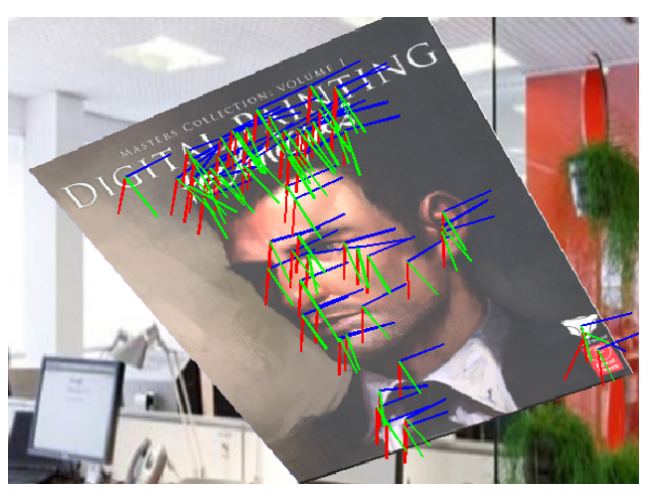

(c)

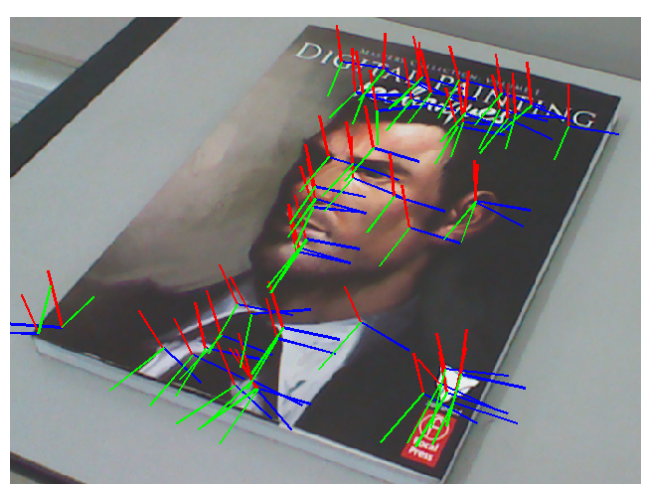

(b)

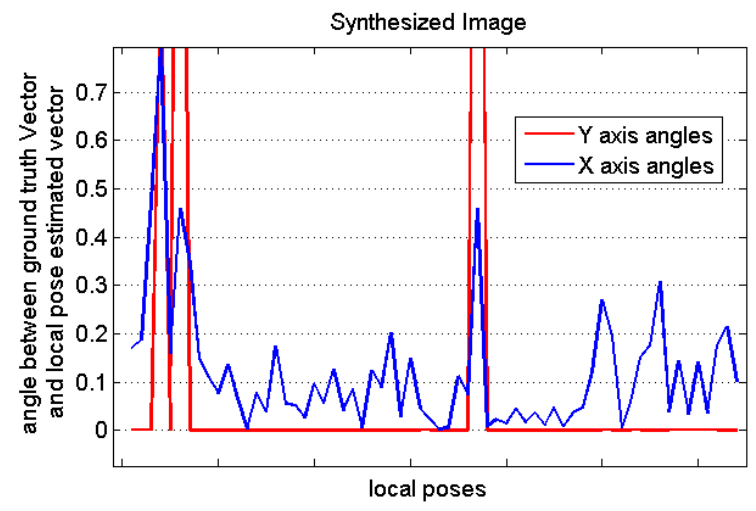

(d)

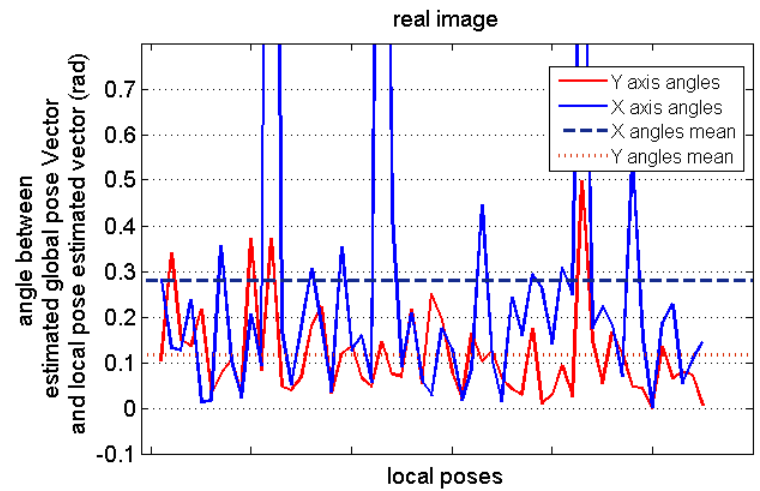

Source: Author.

$\mathrm{X}$ axis calculated using the local pose $\mathbf{T}_{\text {local }}^{i}$ as $X_{\text {local }}=\mathbf{T}_{\text {local }}^{i}[1 ; 0 ; 0 ; 0]^{T}$, and $X_{\text {groundTruth }}$, the $\mathrm{X}$ axis calculated with the ground truth pose of object. Similar calculation is applied to calculate the $\mathrm{Y}$ axis.

From 39b we can observe that error in Y-axis does not exists for a major number of local poses, due to noise free synthesized depth map. However, errors across X-axis are observed. This is created by the small errors of the orientation vector $\vec{o}_{f t s}$ projection across different views, as presented at 7.3.1.

In figure $39 \mathrm{~d}$ we show the angle oscillation of the axis compared to the estimated pose using 7.3.1. In this case the oscillation in Y-Axis also appears, due to the depth map imperfections and noise. However, its mean value is still smaller than $\mathrm{X}$ axis oscillation.

Another issue of our RGB-D local pose estimation method is that, it is strongly related with near surrounding surface of the feature point. This makes the local pose vulnerable to local noises, specially depth map noises, and this local pose noises can lead 
to larger errors in the global pose. In order to study the effect of depth map noises and orthogonality of the locally planar feature points to the camera, we have proceed a test with synthesized images.

In order to proceed this test, we have generated a similar test setup asLepetit, Moreno-Noguer e Fua (2009). Here, we have generated 300 images of [1280×960] resolution and its corresponding depth map with the planar object placed in different random poses, using a virtual calibrated camera with focal length of $\left(f_{u}, f_{v}\right)=(1249.59,1294.83)$ and a principal focal point at $\left(u_{c}, v_{c}\right)=(640,480)$. The object was placed at 900 millimeters away from the camera and a random translation and rotation is applied. However, the object always is facing the camera, not showing its back face, and also, is always inside the camera field of view. For each depth map, noises are added using Gaussian noise with mean equal to 0 and standard deviation varying from $[0,15,30,45,60,75] \mathrm{mm}$, and then, a low pass filter, implemented as a $3 \times 3$ frame mean convolution, is applied to simulate a AR system with noise and its filter .

As in (LEPETIT; MORENO-NOGUER; FUA, 2009), rotation error is measured as $E_{\text {rot }}(\%)=\left\|q_{\text {true }}-q\right\| /\|q\|$, and translation error as $E_{\text {trans }}(\%)=\left\|t_{\text {true }}-t\right\| /\|t\|$, where $q_{\text {true }}$ is the known true rotation of the object $R_{\text {true }}$ converted to normalized quaternion and $q$, the measured rotation $R$ converted to normalized quaternion. As well as, $t_{\text {true }}$ is the known true rotation, and $t$ the measured rotation. $\mathrm{X}$ and $\mathrm{Y}$ axis error angle are calculated as in figure 39.

Finally, plane orthogonality is calculated by the angle between the normal of the plane and $\mathrm{Z}$ axis, calculated as ortho $=\operatorname{acos}\left(\mathbf{T}_{\text {groundTruth }} \vec{n}_{o b j} \cdot[0 ; 0 ; 1 ; 0]^{T}\right)$. Here, larger angles means that the plane is more tilted in relation to the camera, meaning that is less orthogonal to the camera. Figure 40 show an example of how the depth map noises appears and estimation results of the local poses.

This figure illustrates the effect of the depth map noise in the local pose error, as discussed above. It is possible to observe from 40e and $40 \mathrm{f}$ that small noises already creates strong rotation error in $\mathrm{Y}$ axis calculation, generating rotation errors in the entire local pose. Although real conditions using our test setup do not create noise levels as we used in this test, as is possible to see in real scenarios local pose results as in 38 , we push the noise level in order to analyze the noise effects over the local pose estimation method. Figure 41 shows the box plot of the estimation errors in local poses across depth map noises and across orthogonality of the plane to camera direction.

These figures highlight the influence that depth map noise has in estimation errors of local poses. It is possible to observe that the error increase is higher when the depth map noise is varied, as in the first row of 41, instead the orthogonality of camera, in the second row. This influence is specially higher in the Y-axis error, illustrated in figure41d, leading to greater rotation error, figure 41a, as spotted above. In figure $41 \mathrm{~d}$ is possible 
Figure 40 - Depth maps with noise and result of the local pose estimation based on RGB-D. First row: depth maps with Gaussian noise added with sigma = $[0,15,45,75] \mathrm{mm}$. Second row: respective estimation results of local poses using the depth map above it.

(a) depth map noise $=(\mathrm{b})$ depth map noise $=(\mathrm{c})$ depth map noise $=(\mathrm{d})$ depth map noise $=$
$0 \mathrm{~mm}$ $0 \mathrm{~mm}$

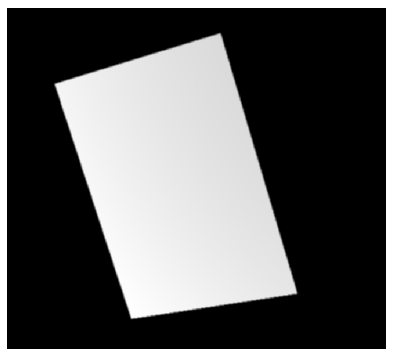

(e) local pose estima-(f) local pose estimation with depth noise $=0 \mathrm{~mm}$

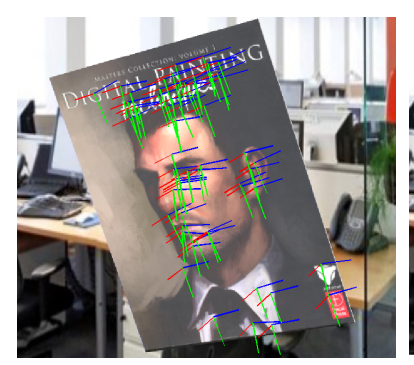

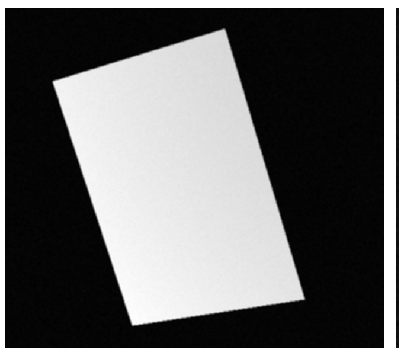

tion with depth noise $=15 \mathrm{~mm}$

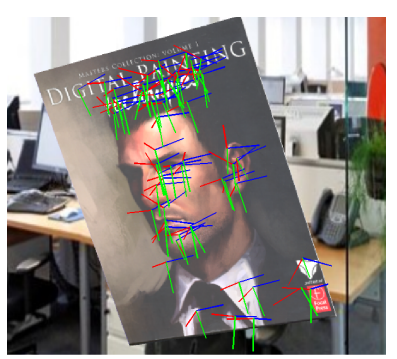

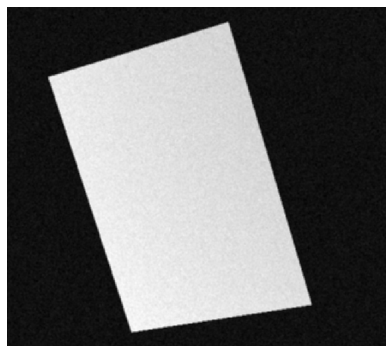

(g) local pose estimation with depth noise $=45 \mathrm{~mm}$ $75 \mathrm{~mm}$

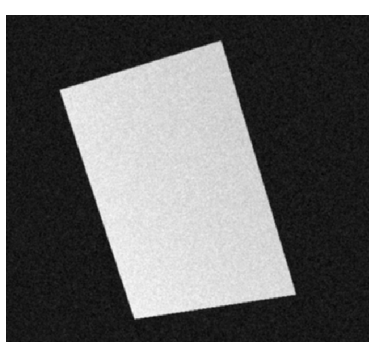

(h) local pose estimation with depth noise $=75 \mathrm{~mm}$

Source: Author.

to observe that the Y-Axis error is almost 0 in conditions without depth map noise and suddenly increase when noise is added.

All of this effect is related with the normal calculation done in equation (7.9) in chapter 7.2. Once normal $\vec{n}_{\text {obs }}$ (which is equal to the Y-axis in planar objects) is calculated by a vector cross product of 2 vectors over the object surface, if these vectors has rotational errors, they lead to a noisy normal vector, leading also to a noisy local pose. This effect could be attenuated by calculating this normal in a different way, which we will discuss latter in section10.2.

Also, it is possible to observe another interesting effect from these images. From image $42 \mathrm{~d}$, we can observe that, although the deviation is increasing, the median value of the error is decreasing with the orthogonality decrease (i.e. the value of the angle increase). This could be counter-intuitive, once orthogonality decrease indicates less visibility of planar object, that can leads to greater pose errors. However, this effect can also be explained by equation (7.9).

Again, once normal $\vec{n}_{o b s}$ is calculated by vector cross product, when the plane is more orthogonal to camera, one of the vector can have longer length in $3 \mathrm{D}$, even having 
Figure 41 - Box plot of local poses errors across depth map noise and camera orthogonality. First row: errors across depth map noises with random camera orthogonality; second row: errors across camera orthogonality with random depth map noises. First column plots the rotation error in \%; second column, the translation error in \%; third column, $\mathrm{X}$ axis error angle between local and ground truth in rad; fourth column, Y axis error angle between local and ground truth in rad.

(a)

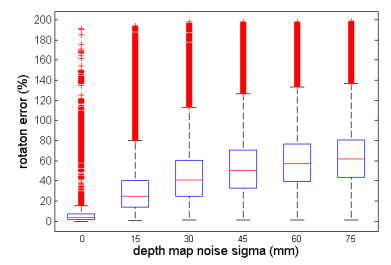

(e)

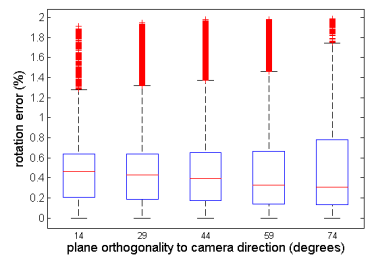

(b)

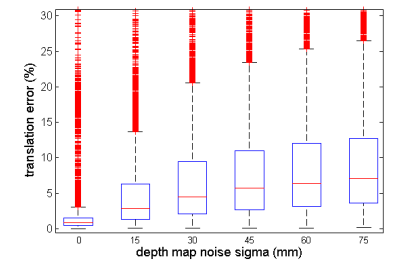

(f)

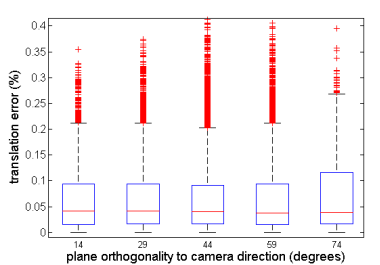

(c)

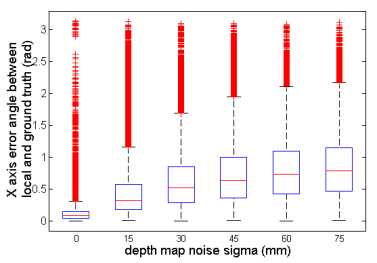

$(\mathrm{g})$

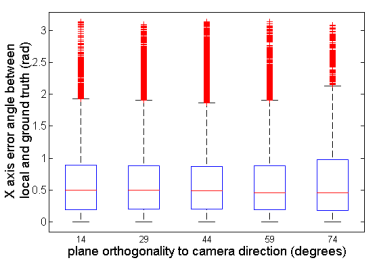

(d)

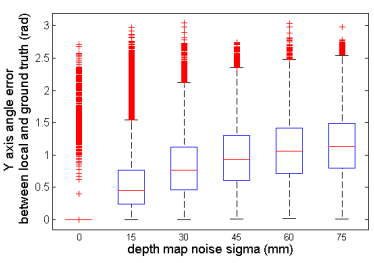

(h)

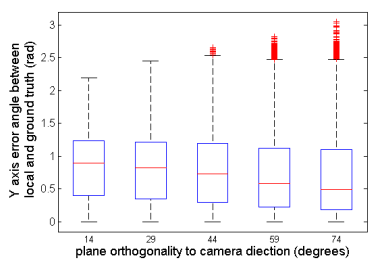

Source: Author.

small length in the projected 2D image. This larger vector length makes it more robust to local noises, shown at (LI; XU; XIE, 2012).

It is possible to observe that this normal error decrease has also direct impact to the object rotation error, as presented in figure $42 \mathrm{a}$. In figure 42 we present the median plot of the errors across the two variables, using a surface plot.

These figures make clear the effects pointed in the other images. From $42 \mathrm{~d}$ is possible to analyze that depth noise is strongly affecting the error, but these errors has a decreasing with the othogonality increasing. This effects in Y-axis is also visible in other errors, as in rotation error figure42a. In section 9.2.1 we show how these local pose errors affects the global pose estimation process.

Results of local pose estimation, based on RGB-D data, obtained by 3D object poses using real information are also shown at figure 43. In these images, we show the estimated local pose from $3 \mathrm{D}$ object with and without the conversion of the local information to 3D object using $f_{o b j}\left(T_{t e x}\right)$, as shown in chapter 7.2.

This conversion $f_{\text {obj }}\left(T_{t e x}\right)$ function converts the normal vector, Y-axis, of the locally planar feature point to the object space Y-axis. From the first row of 43, which shows the not converted poses, we can observe how the normal calculation can be done when the object is not planar. Even that locally planar assumption is not totally true, still the 
Figure 42 - Surface plot of depth map noise and orthogonality interaction effect in local pose errors.

(b) depth map $\times$ orthogonality $\times$ translation er-

(a) depth map $\times$ orthogonality $\times$ rotation error ror

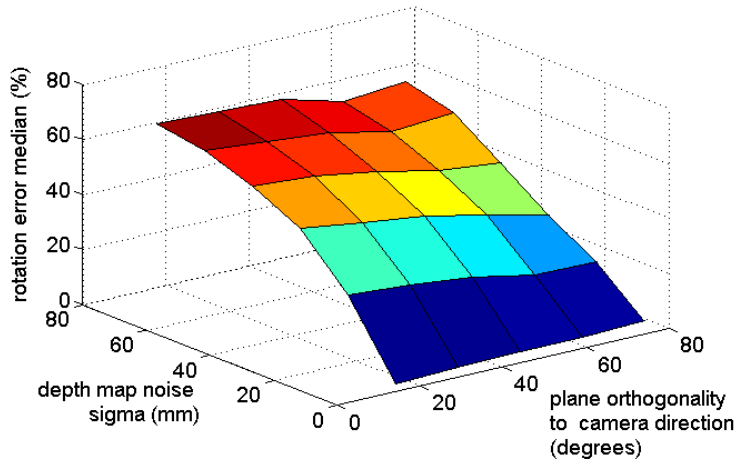

(c) depth map $\times$ orthogonality $\times \mathrm{X}$ axis error

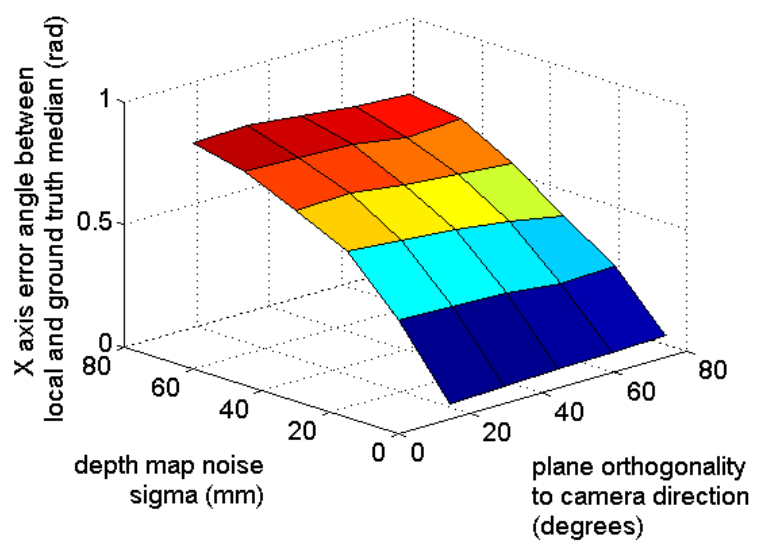

Source: Author.

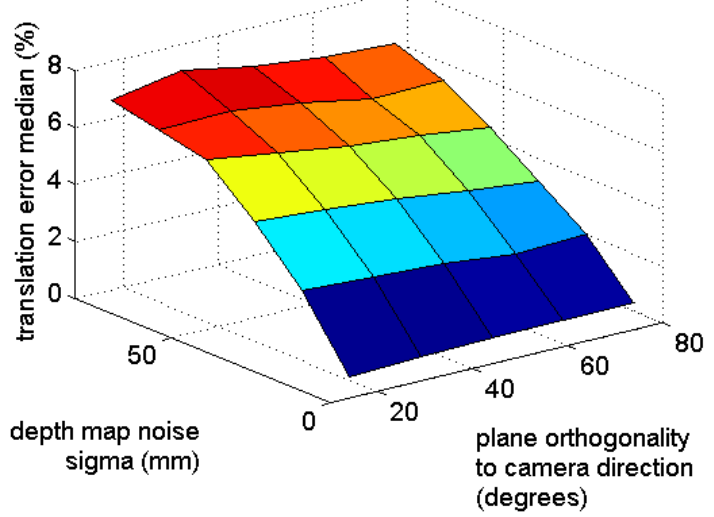

(d) depth map $\times$ orthogonality $\times Y$ axis error

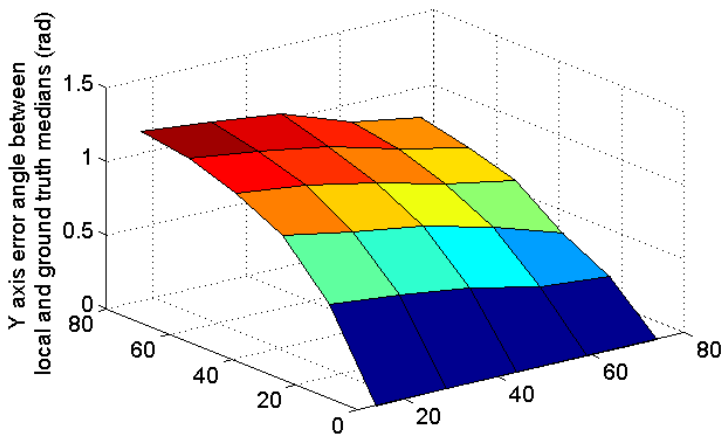

estimation of local pose can be done, as shown at second row of 43 .

However, also from these images, is possible to observe the weakness of breaking this assumption. We can observe that some detected feature points at the edge of the object has wrong local poses. These errors are due to the curvature of the feature points that are partially observed by the camera. Therefore, when the local pose are calculated the normal and direction vector are not calculated perfectly, once they are not fully observed. Yet, they can be filtered in the global pose estimation process as we show in the next section.

Figure 44 shows the results with deformable objects in different poses. Similarly to 3D objects, the local pose can be calculated even with the deformations. In the next section we will present the estimated global poses, based on local pose information captured using RGB-D cameras. 
Figure 43 - Local pose of 3D object calculated with our RGB-D local pose method. First row: poses without the object global pose correction done by $f_{o b j}\left(T_{t e x}\right)$; second row: correct local poses to object poses.
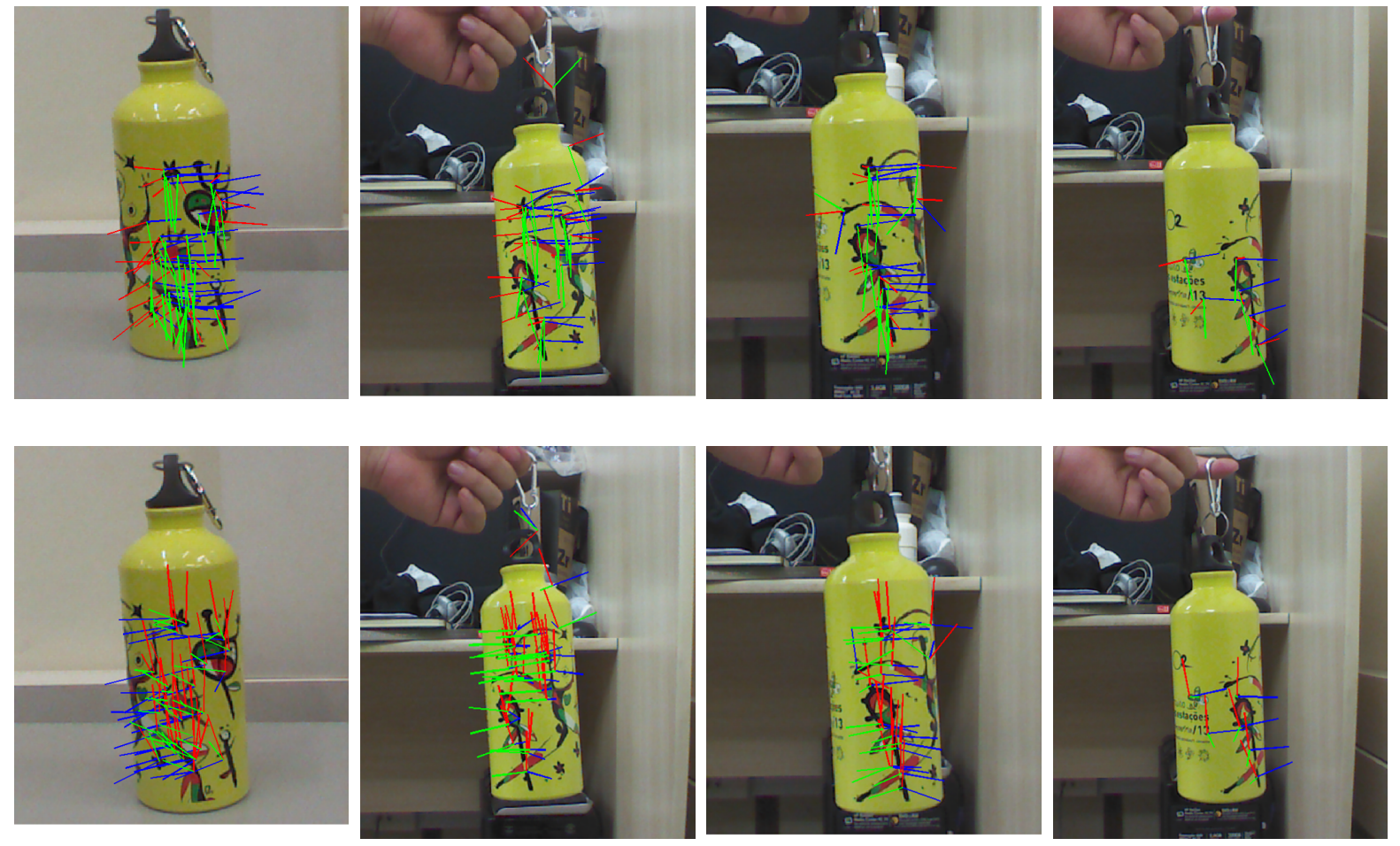

Source: Author.

Figure 44 - Local pose of deformable objects.
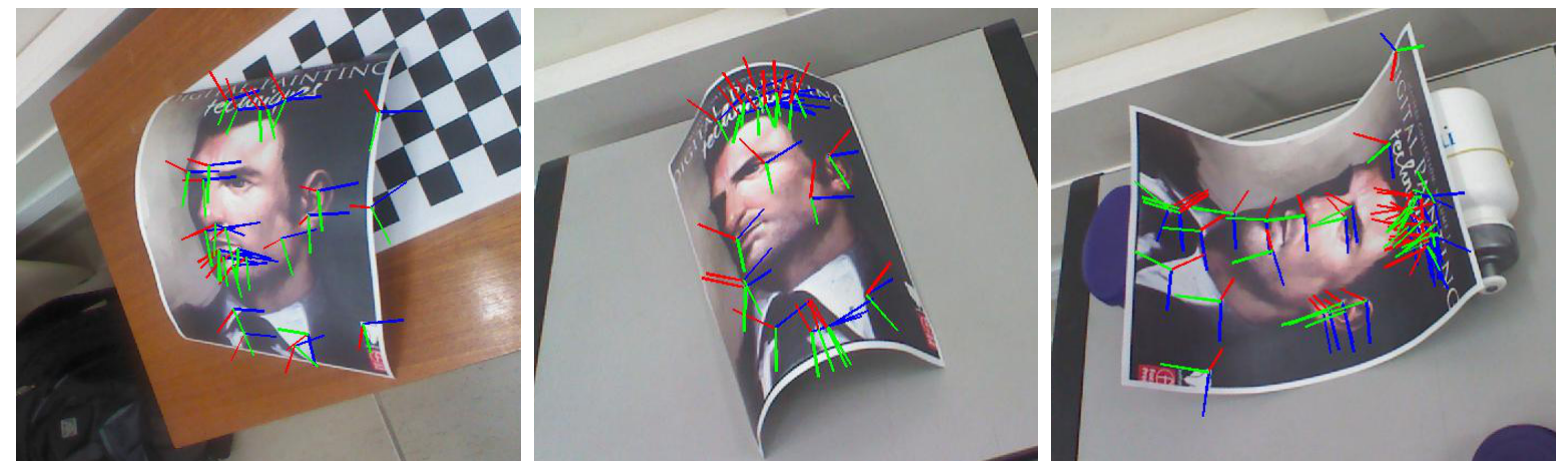

Source: Author.

\subsubsection{Rigid Object Pose Estimation}

Here, we present the estimation results of global poses obtained by using rigid objects as markers, planar and 3D. Results shown below are obtained by using our RANASAC based method, section 7.3.1. In these results, the red lines represents the estimated object itself, which have exact size of the estimated object. In figure 45, results obtained by planar object are shown. 
Figure 45 - Results of global pose estimation method for planar objects obtained by RANSAC method. Results obtained using real images with camera positions less orthogonal to object plane from images $45 \mathrm{a}$ to $45 \mathrm{~d}$. There results are obtained by local poses estimated at figure 38 .

(a)

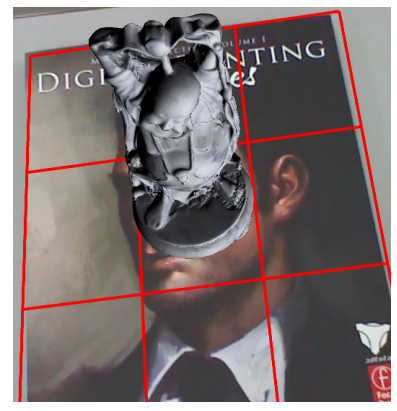

(b)

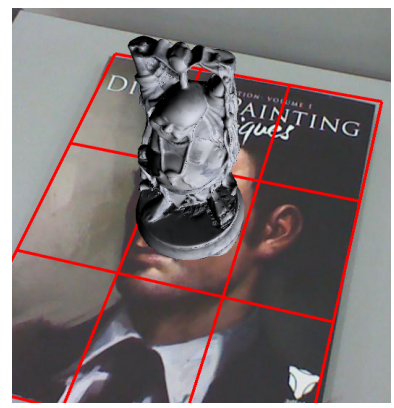

(c)

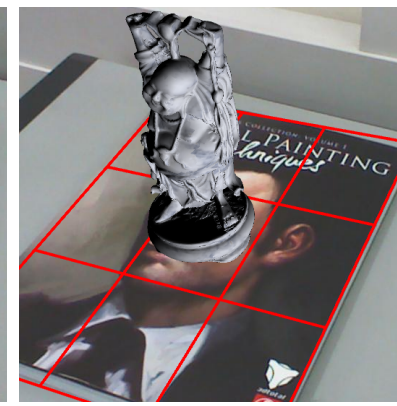

(d)

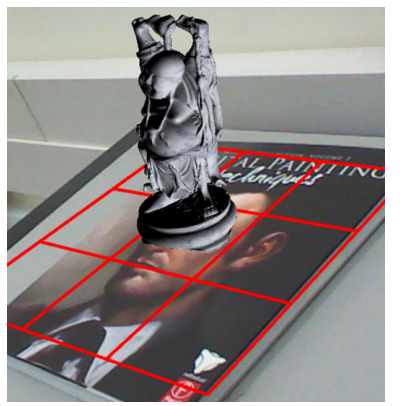

Source: Author.

Figure 45 shows the global poses of planar objects estimated by the local poses obtained in figure 38. From these results in red grid, which represents the estimated object poses, is possible to observe that object pose accuracy decreases with camera orthogonalities decrease. This result goes against the result presented at figure 41, yet, can be explained by the decrease of number of detected features points, as shown at 35, and the precision decrease of local pose rotation in $42 \mathrm{a}$, represented with the standard deviation increase. Therefore, as more orthogonal to camera, the number of detected features decrease, giving less clues to estimate the object poses, giving less accuracy.

We also apply the same RANSAC global pose estimation method on cylindrical objects. The estimation results are shown in figure 46. From the results presented in figures 45 and 46 it is possible analyze that results returned by our local pose approach are suitable for $\mathrm{AR}$ registration in case of rigid objects, planar or $3 \mathrm{D}$.

\subsubsection{Synthesized Data}

In this subsection, we show the evaluations of our global pose estimation method using the local poses estimation approach. Here, in order to evaluate our approach for the planar objects, we compared our approach to conventional RANSAC approach based on plane calculation from triangles formed by each three observed points, as in (HENRY et al., 2012). As in section 9.2, we evaluate the errors by creating 300 images with same conditions.

Also, as in section 9.2, we generate several depth maps with Gaussian noise with standard deviation varying from $[0,15,30,45,60,75]$ millimeters $(\mathrm{mm})$. Furthermore, in this evaluation, $2 \mathrm{D}$ noise is also added to the detected feature position at the image space as a Gaussian noise with mean 0 and deviation varying from $[0,6,12,16,24,30]$ in pixels $($ pix $)$. Rotation and translation error are measured as in section 9.2 and Lepetit et al. work 
Figure 46 - Results of global pose estimation method for cylindrical 3D objects.
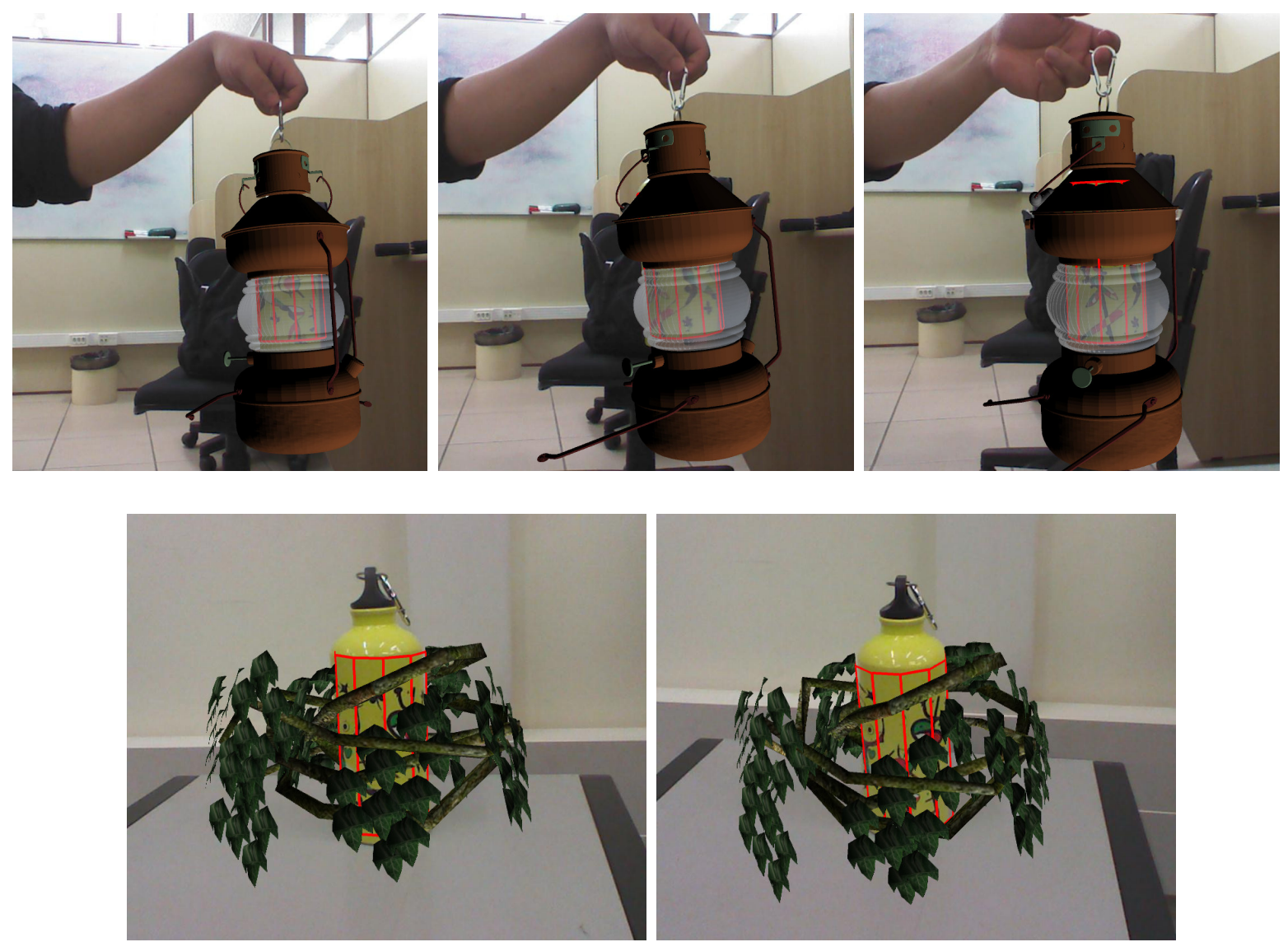

Source: Author.

(LEPETIT; MORENO-NOGUER; FUA, 2009). We present the translation and rotation error in Figure 47 and 48 with a box plot representation, across different depth map noises, figure47, and 2D position noises, figure 48.

Observe that our method has lower accuracy and higher variation with depth map noise, compared to conventional three-point RANSAC method. This is due to the fact that our method is based on local information, where small depth noises can generate big errors in the global pose, specially in rotation, as presented above at section 9.2. However, when the noise increases, our method has better accuracy, as well as, lower variance in comparison to conventional method. For 2D noise case, our approach is more accurate for small 2D errors compared to three-points based RANSAC, since the surface around the feature point itself is not changing.

Furthermore, from the process time plots in figure 49, it is possible to observe that our method has a slow increase in process time across both type of noise values. However, the process time of the conventional three-point detection method rapidly increases with both types of noises. This is due to the failure of the RANSAC method. 
Figure 47 - Box plot evaluation of our method and conventional three-points based RANSAC pose. Results across different depth map noise. Upper row: rotation error; second row: translation errors. First column: our local pose approach; second column: three-points based RANSAC.

(a)

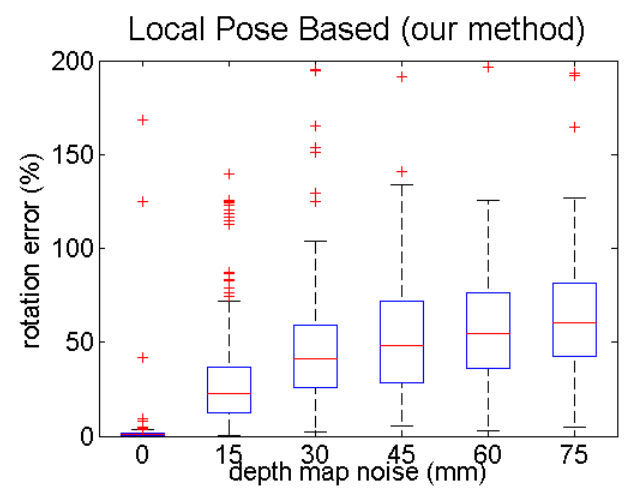

(c)

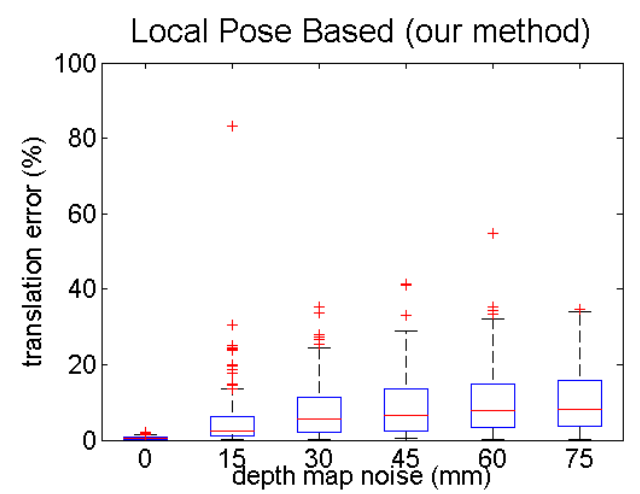

(b)

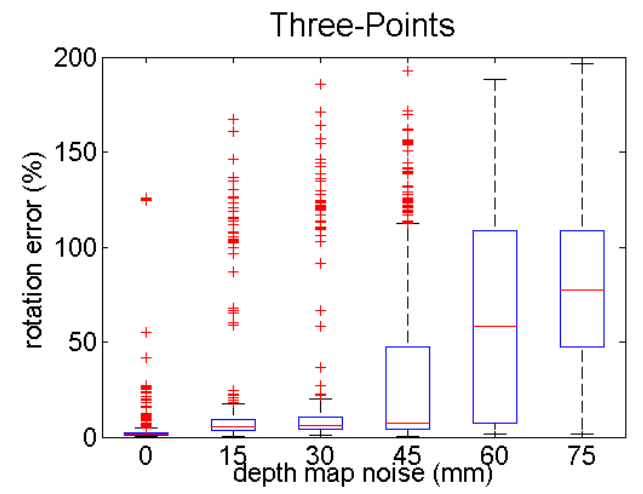

(d)

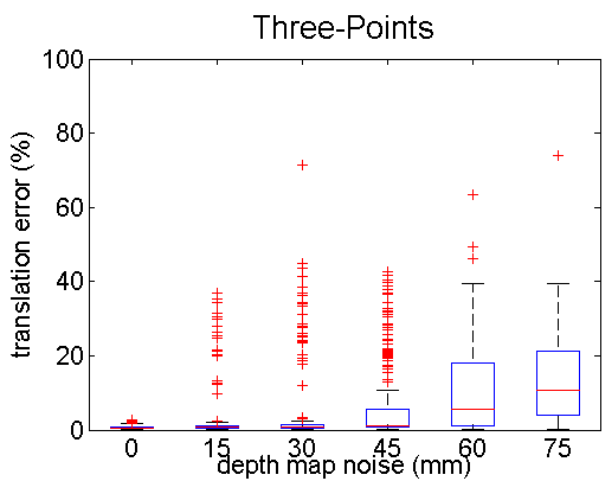

Source: Author.

In this setup, the RANSAC error thresholds are fixed with values empirically found to return good estimation results across different poses. As the error increases, the tests rejection cases of RANSAC also increase, leading to more trials. As presented at section 7.3.1, since our method is based on the local information of each feature point found, the total number of trials is equal to the total points found number $n_{f}$ and induced errors only affect each point. On the other hand, the three-points method uses a combination of each 3 points, meaning that has $\left(\begin{array}{c}n_{f} \\ 3\end{array}\right)$ of total trials, also, each noisy point affects a large number of trials. If $m$ points are affected by noise, only $\left(\begin{array}{c}n_{f}-m \\ 3\end{array}\right)$ are correct. Leading to a large number of failures, and thus to a larger process time.

In order to analyze the effect of the RANSAC error threshold, we evaluated the mean rotation error response across different depth map noises, varying from $[0,15,30,45,60,75]$ millimeters $(\mathrm{mm})$, and RANSAC error thresholds, varying from $\left[5^{2}, 85^{2}\right]$ in $\mathrm{mm}^{2}$. We present these results and the process times in Figure 50.

From this result, it is possible to observe that the three-points based RANSAC has 
Figure 48 - Box plot evaluation of our method and conventional three-points based RANSAC pose. Results across $2 \mathrm{D}$ noise of the feature point position in the image space. Upper row: rotation error; second row: translation errors. First column: our local pose approach; second column: three-points based RANSAC.

(a)

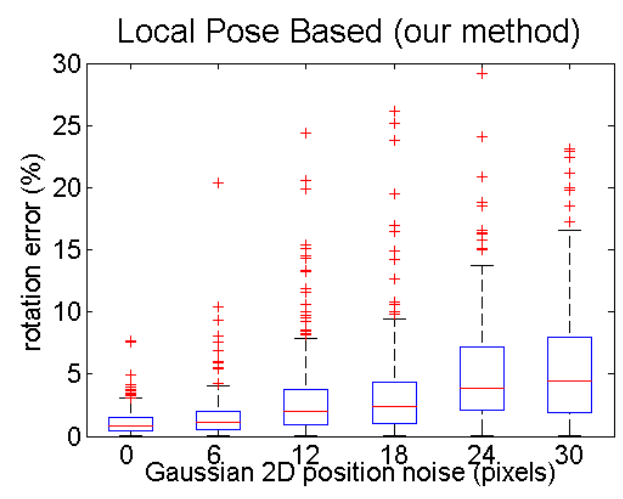

(c)

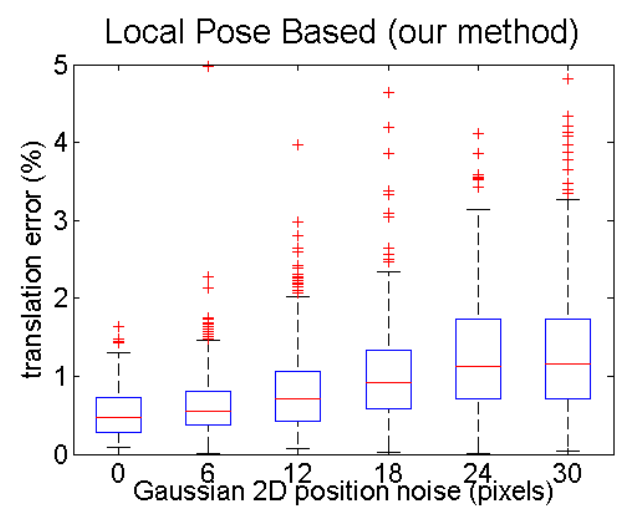

(b)

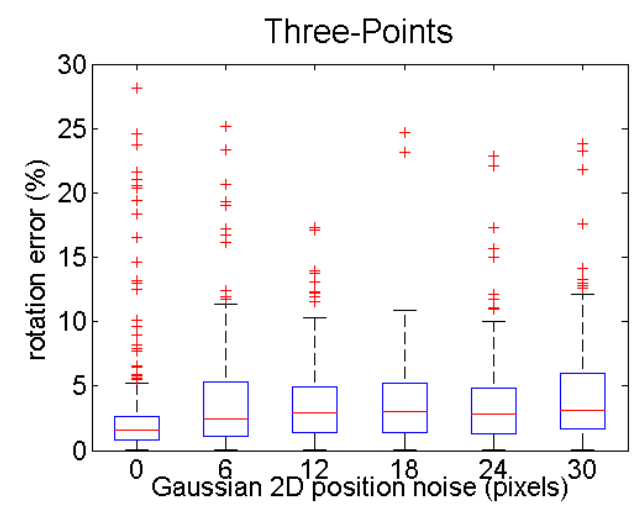

(d)

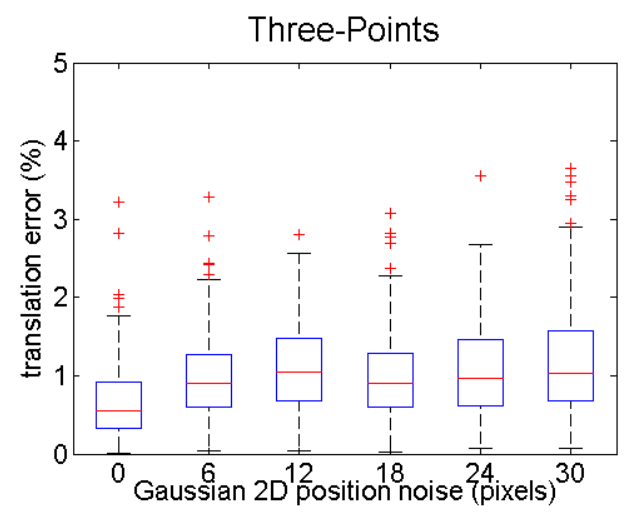

Source: Author.

a sudden increase in process time with the RANSAC error threshold decrease. However, from the mean error, it is possible to observe that it also has a sudden error increase with noise or RANSAC error threshold increase. This property allow to observe that the three-points based RANSAC is more sensitive to settings. In contrast, our local pose based approach does not present this characteristic, showing almost constant process time increase through RANSAC error decrease and noise increase, as well as, slow error increase through noise and RANSAC error threshold increase.

\subsubsection{Single Feature Pose Estimation}

One interesting characteristic is that the global pose of rigid objects should be equal to all the local poses, as presented in section 7.3.1. This characteristic leads us to a novel solution of pose estimation, the estimation of the object pose by a single detected feature point. This results is novel due to the fact that other pose estimation based on 
Figure 49 - Mean process time across added error noise values.

(a) process time across depth noises

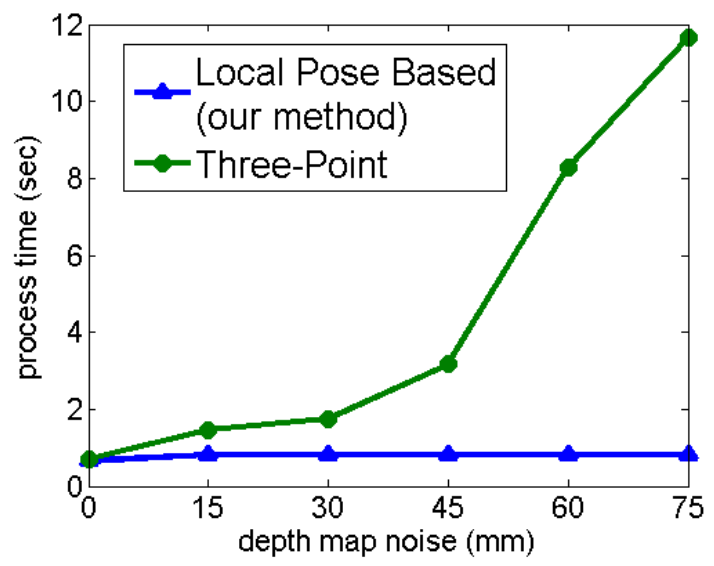

(b) process time across $2 \mathrm{D}$ position noises

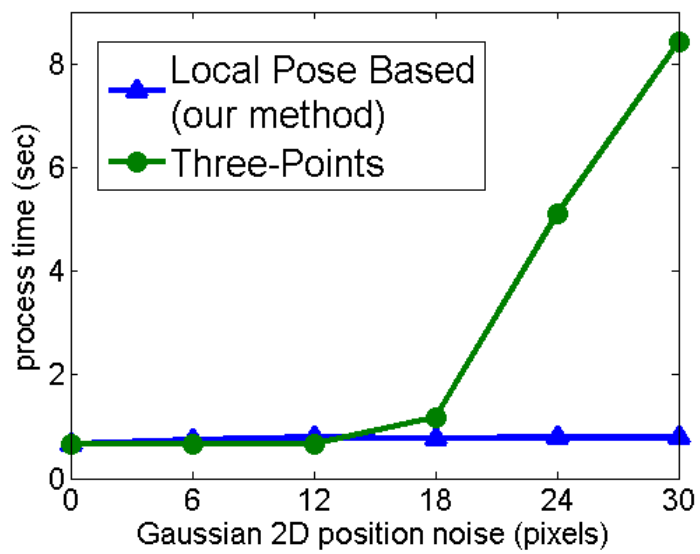

Source: Author.

features points, including methods based on depth information or solve-P $n \mathrm{P}$, presented in chapter 3 , requires 3 interest points at least in order to estimate the object pose.

In order to make clear, our method does not achieve the global pose only by a single known point, and still needs at least 3 points to estimate the object pose. However, the main difference in our approach is that all the 3 needed points can be extracted by only a single known feature points, by considering the feature point position itself as a first point, the texture major orientation vector as a second, and the orthogonal point to texture orientation as the third point. Furthermore, we can analyse that this result is achieved by the fact that we extract local information, instead of considering each feature point as just a point, unlike other works. Figure 51 present results of pose estimation based on a single feature point.

Although, this single feature point pose estimation can be achieved, the major problem here turns to be the feature point detection/matching process itself. As show at section 9.1, one feature point does not appear in all the possible view, instead, only in a limited number of views. In case that the desired feature point is not detected, the pose can not be estimated. Another problem is in the estimated pose accuracy. As shown in 39 and 41, the local poses can have small errors compared to the accurate global pose, specially in presence of depth map errors.

Despite these problems, the single feature point pose estimation can be explored in several cases as support tool in pose estimation, or in combination with other methods in order the obtain full pose estimation or tracking. These side usage of our results in single feature point pose estimation will be discussed with more detail in chapter 10 . 
Figure 50 - Evaluation of our method and three-points based RANSAC, across RANSAC error threshold and depth map noise. Upper row: rotation error. Lower row: process time.

(a) rotation error across depth noises and (b) rotation error across depth noises RANSAC settings of our local based approach.
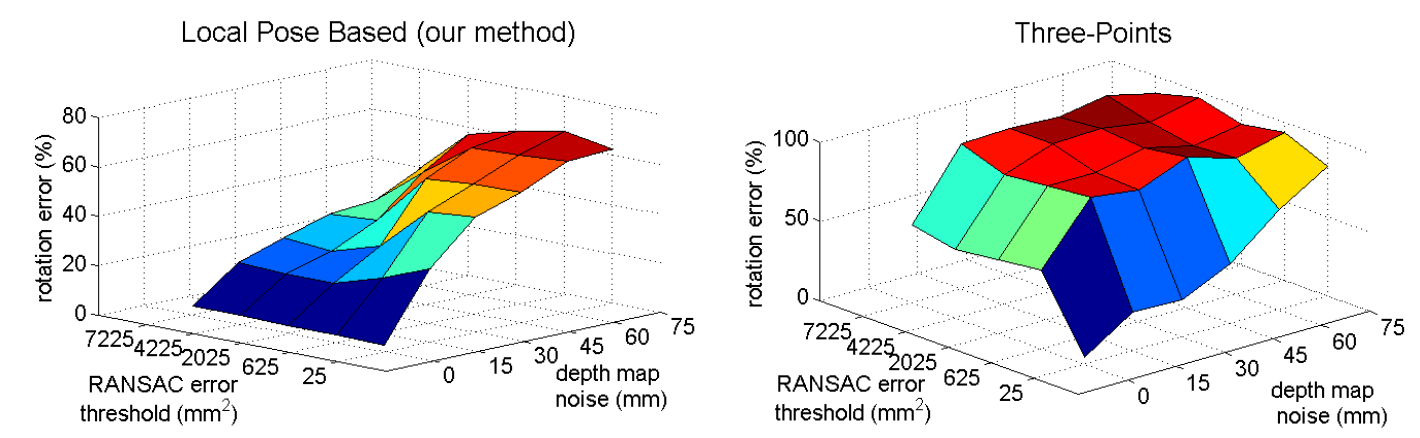

(c) process time across across depth noises and RANSAC settings of our local based approach.

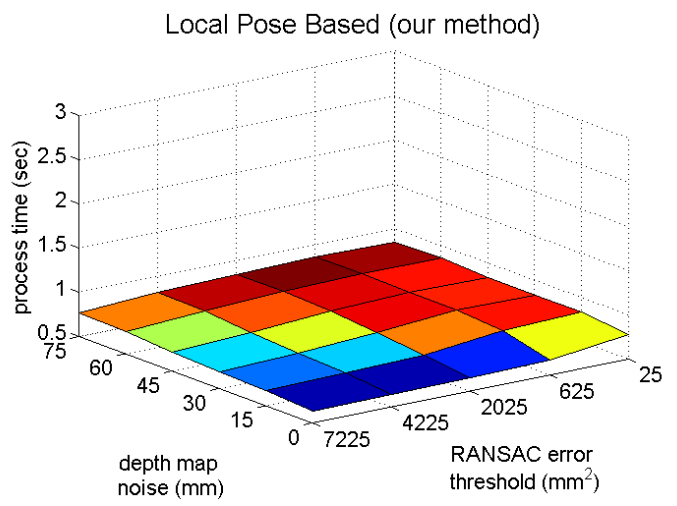
and RANSAC settings of three-points RANSAC.

(d) process time across across depth noises and RANSAC settings of three-points RANSAC.

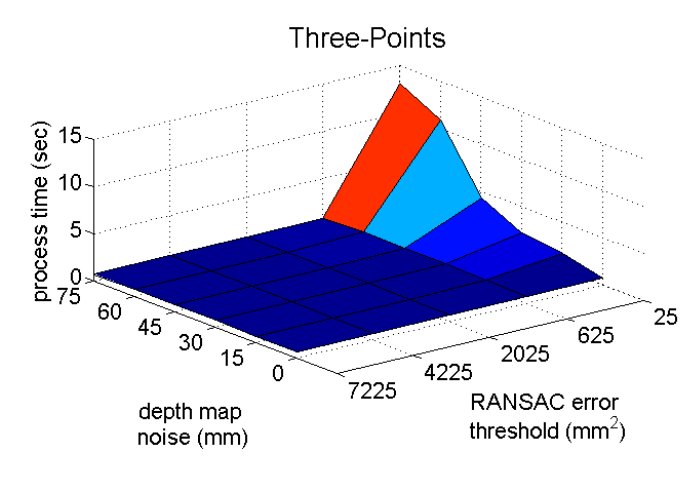

Source: Author.

\subsubsection{Deformable Object Rough Pose Estimation}

A final piece of our global pose estimation using local poses based on RGB-D cameras is the rough pose estimation of deformable objects. Here, we use a planar image printed in a sheet of paper as marker and global pose estimation method as presented in section 7.3.2. Figure 52 presents the obtained results.

Figure 53 shows other results obtained by deformable object pose estimation. In this figure, we use the pose of each found feature point in order to render an object, rendering an object in each found feature point.

As in single feature point pose estimation, the deformable object pose estimation also is strongly affected by uncertainties of feature point detection/matching and pose accuracy. In case that some regions of the deformed object are occluded, the registration in these regions will be determined by the nearest found pose, which can be in a different pose leading to wrong registrations. Fails in feature point match also generate wrong 
Figure 51 - Single feature point pose detection results, in different camera view conditions. The estimated local pose coordinate system is also rendered over the detected position.
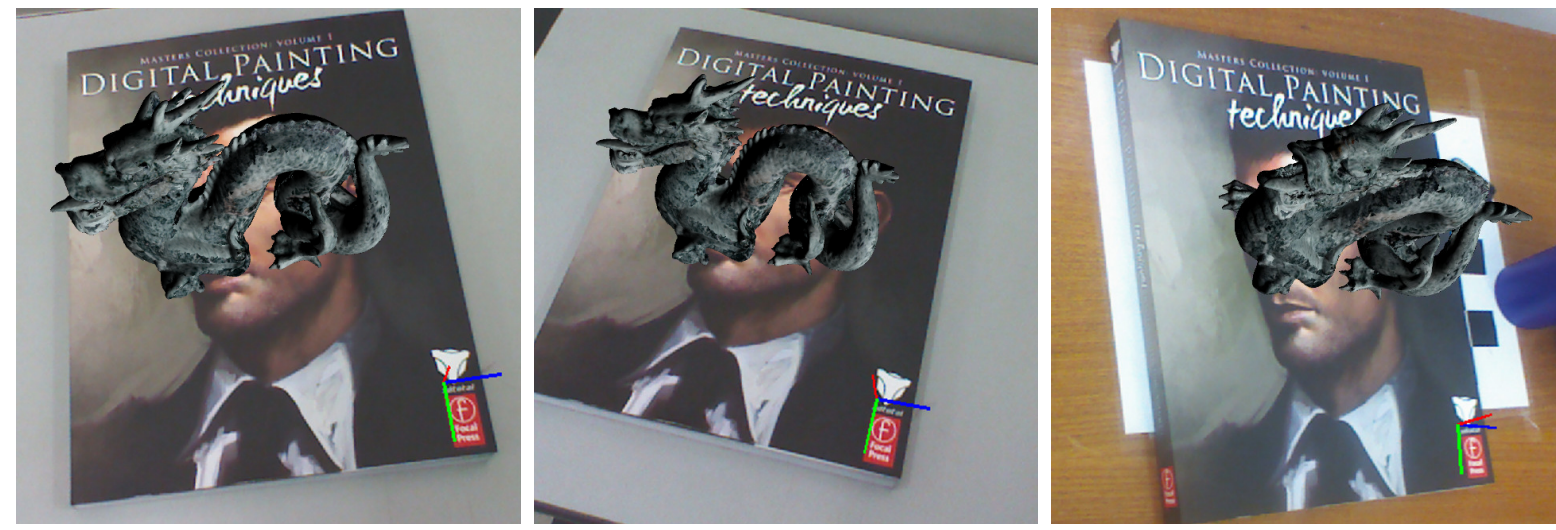

Source: Author.

Figure 52 - Deformable object pose estimation in several conditions.

(a) convex deformation

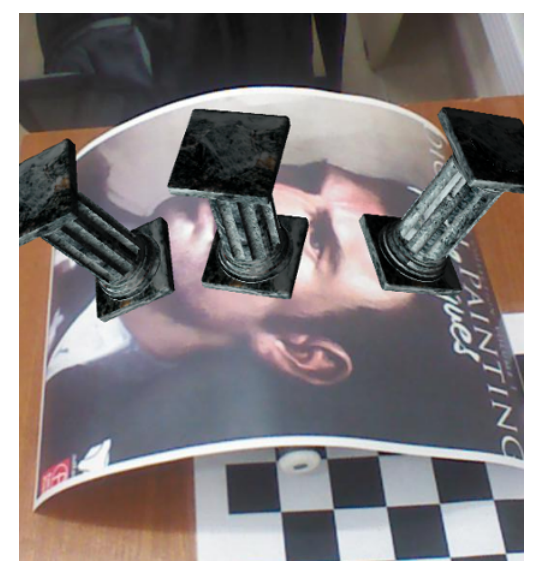

(b) planar - no deformation

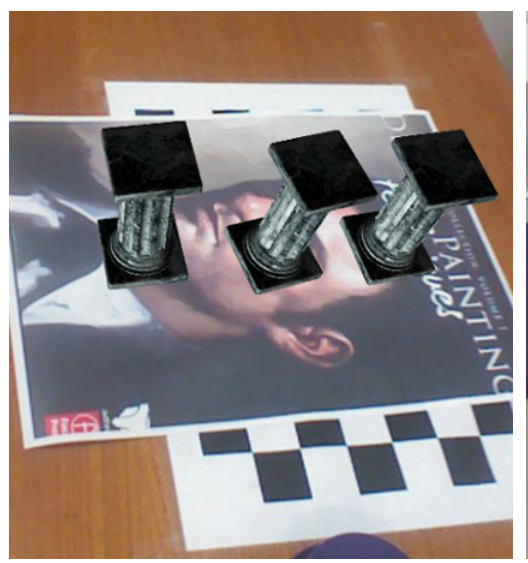

Source: Author. (c) concave deformation

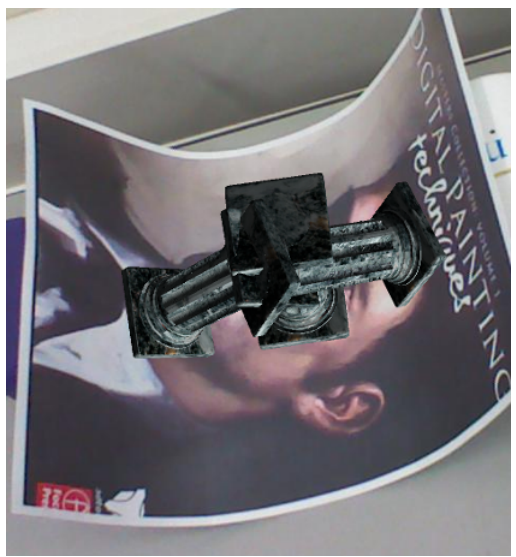

registrations. Finally, as presented in figures 39 and 41, the accuracy of local pose can be a factor of wrong pose registration.

This pose estimation could be improved by combining our local poses with other methods. Also, full pose could be achieved by combining other methods in literature with our local pose approach, as we will present in section 10.5.3. Despite all, rough pose of deformable objects already can be achieved by simply applying our local poses as presented in figures 52 and 53. 
Figure 53 - Deformable object pose estimation, other results.
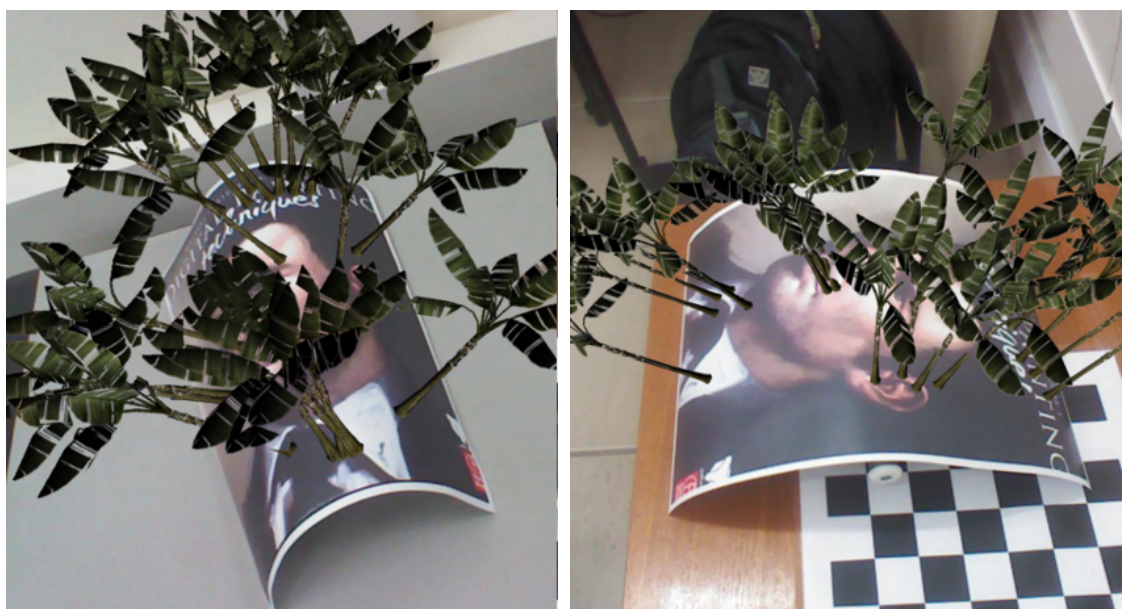

Source: Author.

\subsection{RegressAR: Decision Forest Local Pose Regression based on RGB Information}

Using the same feature points extracted using our method 6, we also can estimate the object pose based on RGB information as presented at chapter 8 . In this section, we present our results of the pose estimation based on RGB information and pose regression forest. At section 9.3.1 we present the results obtained by the regression forest, as well as the estimated local pose. Then, at subsection 9.3.2, results of the pose estimation of rigid objects, obtained by applying our RF-RANSAC method, are presented.

\subsubsection{Regression Forest}

Initially we present our obtained results from the regression forest approach, presented at section 8.2. Figure 54 presents the regression L1 error values returned by several trees in $\theta$ and $\phi$.

We can observe from these images that several trees in the forests return accurate results, specially in $\theta$ regression. In case of $\phi$ regression, also several trees have small error; Despite that, differently from $\theta$, other trees has large errors, around a constant value. This could be caused by similarities of the image patch in different poses.

In order to evaluate the effect of these regression errors, we present the confusion matrix of all the trees in all the matched feature points in 1000 non-trained images with the planar object in different known poses, in figure 55. This confusion matrix is created, first, by choosing 10 equally spaced discrete ranges of the continuous $\theta$ and $\phi$ values. For each evaluation image, we extract the regressed value of $\theta$ and $\phi$ of all the trees of all the matched feature points with decision forest attached. 
Figure 54 - Trees regression L1 errors. Each row show errors returned by different feature points. First column: errors returned by regression of $\phi$ values; and second, by $\theta$ regression.

(a) $\phi$ regression errors.

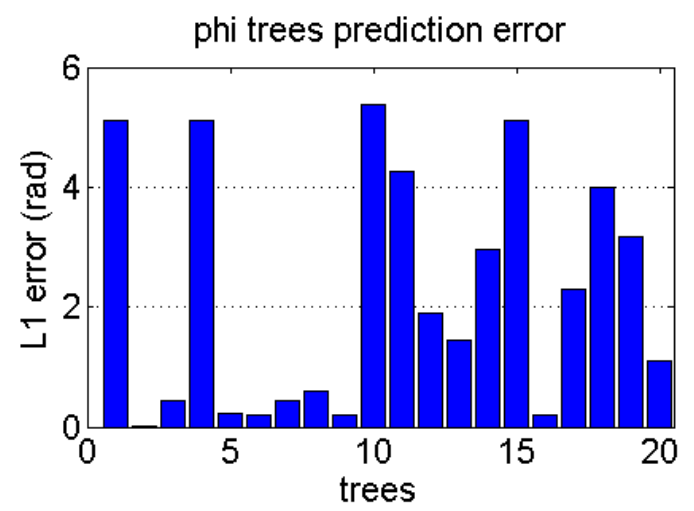

(b) $\theta$ regression errors.

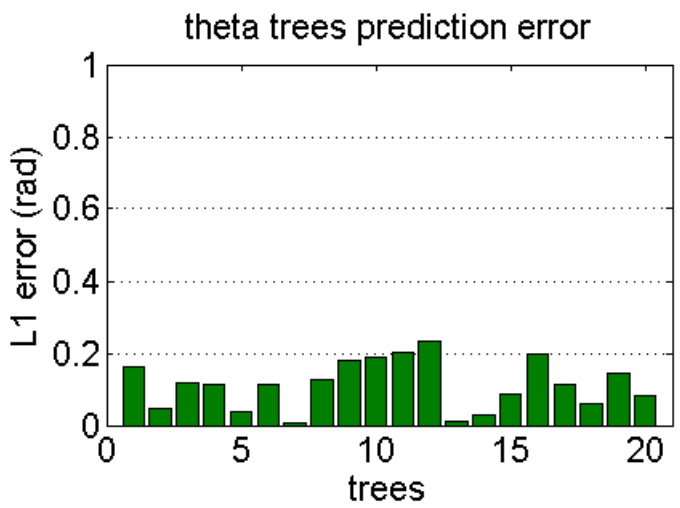

Source: Author.

Then, we convert the continuous ground truth values and regressed into the 10 discrete ranges, also, we count the number of observations in each range. We plot the ground truth values in each row, and the observations in the columns. Each row is divided by each observation count. The diagonal of the plotted matrix represents the probability of true positives, and all the other values are the false positives returned by our regression forest.

Figure 55 - Confusion matrix evaluation with 1000 non-trained images; Y axis represents the ground truth value, and $\mathrm{X}$ axis, the predicted value. Also illustrating the errors in $\phi$ regression.

(a) $\phi$ regression confusion matrix.

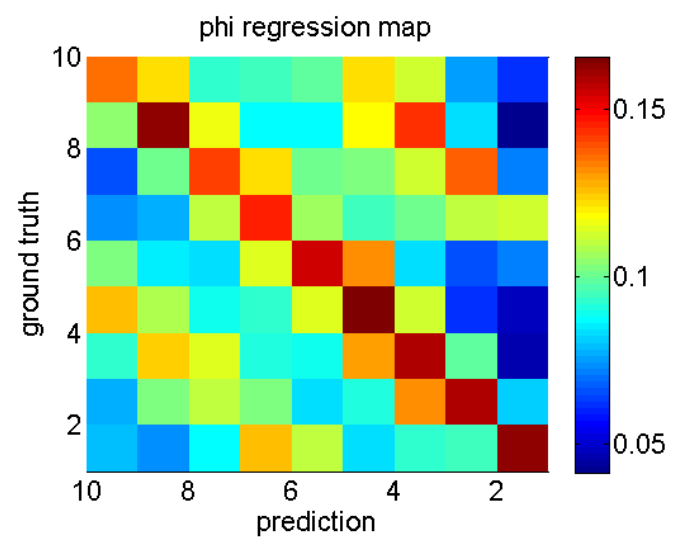

(b) $\theta$ regression confusion matrix.

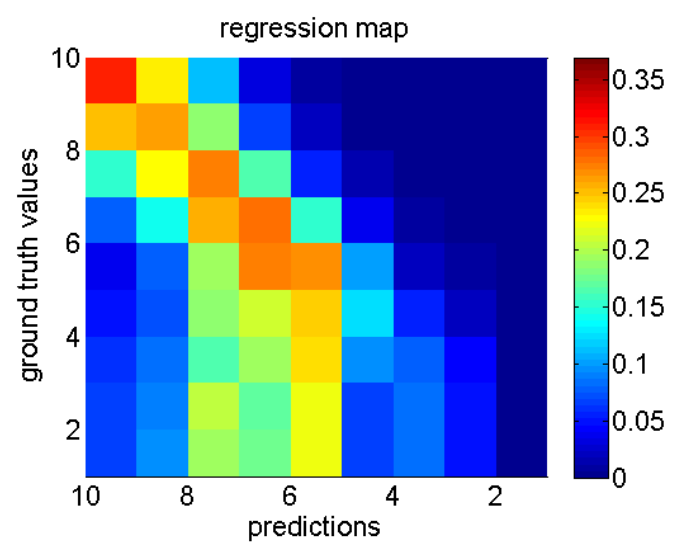

Source: Author.

From figure $55 \mathrm{~b}$ we can observe that $\theta$ regression has small false positives, as shown in figure 54b; until larger $\theta$ values, which means that the object is less orthogonal to the camera direction. This confusion matrix illustrates that as less orthogonal the object is to 
the camera, out approach returns less accurate results.

In figure 55a we present the confusion matrix of the $\phi$ regression values. Differently from $\theta$, can be observed that in almost all the $\phi$ values, there are false positives with $+\pi$ error. This result confirms the result shown in figure 54a, showing that an almost fixed error of $+\pi$ happens to the regression. Thus also showing the necessity of the RF-RANSAC method for pose estimation.

\subsubsection{Rigid Object Pose Estimation based on RF-RANSAC}

We showcase our augmented reality results obtained by RF-RANSAC method in figure 56. Results show in this figure are obtained by using SURF feature (BAY et al., 2008) as features points, with 20 trees of $\tau_{\theta}$ and $\tau_{\phi}$ each, of 20 different feature points. Other than these forest attached feature points, we also use 280 feature points to help in the re-project error calculation and as second point for the rotation and translation calculation. In total, 300 feature points are used for the matching. The observation distance threshold $d_{t h}$ was set to 7 pix, $S 1$ subset percentage $p_{s}$ to be $60 \%$ of entire observation, acceptance percentage $p_{t h}$ as $80 \%$, and $n_{\text {trials }}$ to be 100 .

Moreover, we set as $n_{p i x}=10$ at the feature point extraction size and $l_{f t}=20$; thus we have a training vector $\mathbf{v}$ length of $\mathbf{v} \in \mathbb{R}^{400}$. For training, we have generated more then 2000 images samples with different $\theta$ and $\phi$,with no sigma and position variance, and more 5000 images with totally random poses. 10 different gaussian noise values are added to all of the images, summing a total of more then 70,000 samples used in the each forest training. One important detail is that different cameras with different projection matrix $K_{\text {cam }}$ where used in the first and second row of the figure 56. Thus, showing that our approach demonstrates certain flexibility across different cameras, even strongly based on image appearances.

Although we focused on planar objects here, our approach can be extended for 3D rigid objects. This could be achieved by using the $f_{o b j}$ function. We explore this rigid 3D objects in figure 57 .

Despite further improvement need to be done to increase the accuracy and precision, we observe from 57 , that is possible to extends our method for the $3 \mathrm{D}$ objects. Note also, that this is true even for cylindrical objects. Which, again, breaks our locally planar assumption $A_{o 2}$. This suggests that our assumption can be relaxed for different cases of pose regression also for RGB pose estimation.

\subsubsection{Synthesized Data}

In this section we show evaluations of our method with different setups, in different cases. In order to process this evaluation we have generated 1000 images with random 
Figure 56 - Augmented reality results of planar objects obtained by RF-RANSAC. Red line grids represents the entire object pose estimation. First and second rows results are obtained with different cameras with different projection matrix values.
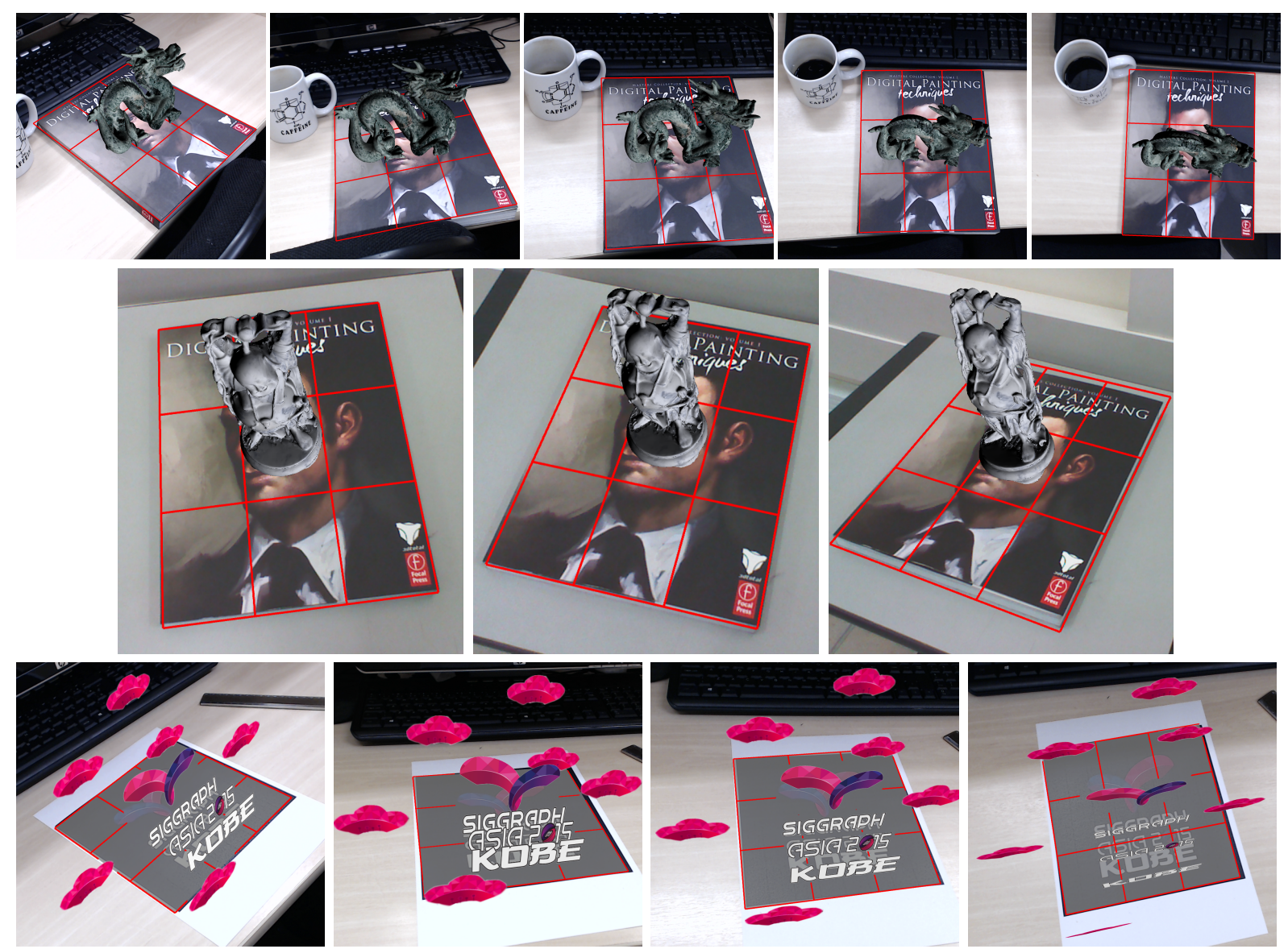

Source: Author.

poses, as in the training part, however, also with realistic backgrounds, set to be photos taken from internet of real environments, in order to simulate a realistic capture.

Additionally, we have generated other 100 images without the background, in order to evaluate the response of all the methods in cases with and without errors in feature points matches. Figure 58 illustrates some samples of the images used here for evaluation, with and without the background.

In figure 59, we show the box plot of the errors obtained by our RF-RANSAC, and also the result of the estimated poses using the full analysis of errors calculated by equation (8.18). Also, in order to evaluate our results, we also compared with EPnP methods proposed by Lepetit, Moreno-Noguer e Fua (2009) and EPnP with RANSAC.

Here, we used the same settings of our method as in the results obtained in figure 56, and used total of 300 feature points for matching, as well as, for EPnP and EPnP-RANSAC methods. Additionally, we also used the same RANSAC settings as our RF-RANSAC in 
Figure 57 - Approximated pose estimation of 3D rigid objects, obtained by extending our approach for $3 \mathrm{D}$ rigid objects.
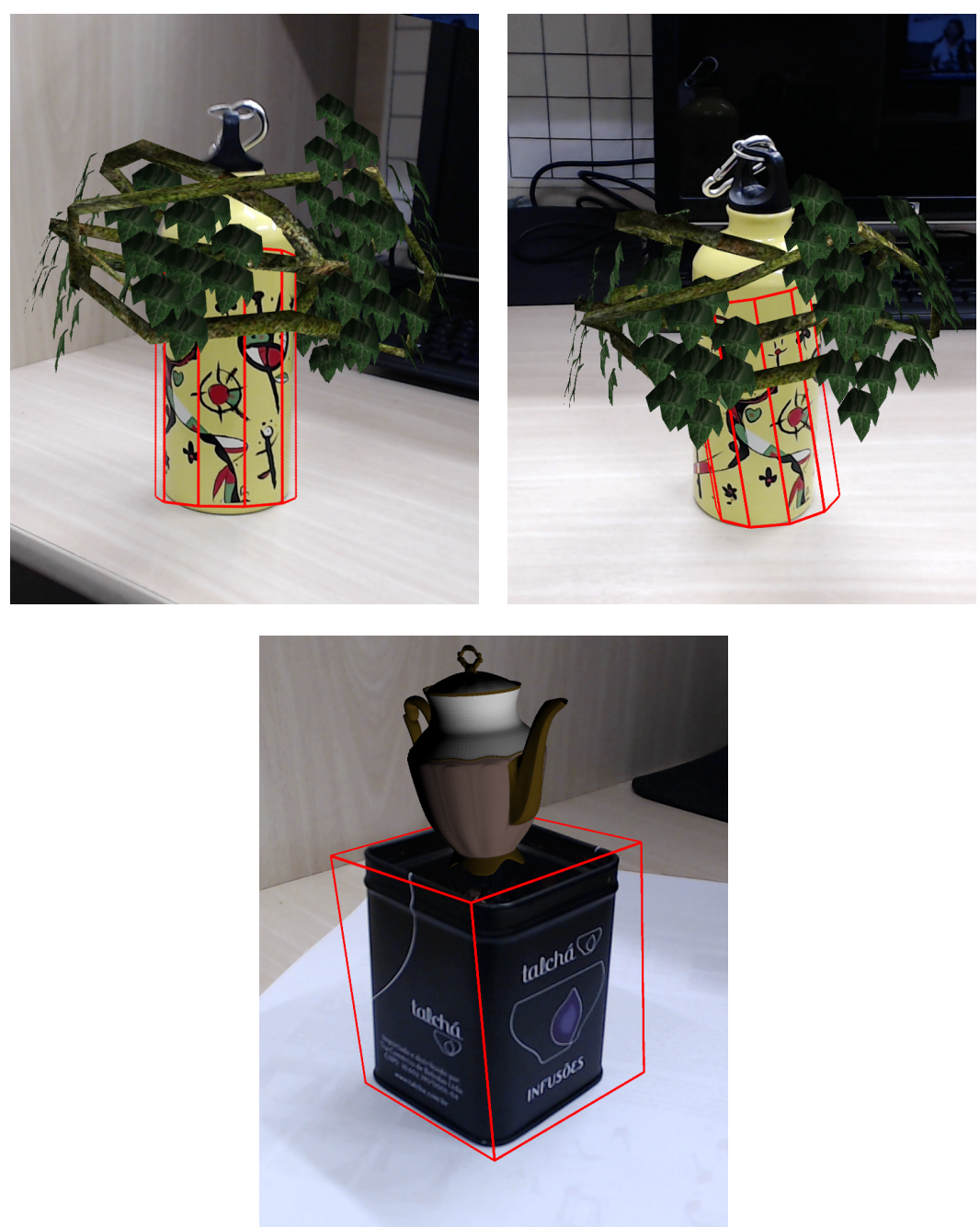

Source: Author.

\section{EPnP RANSAC.}

Error values are calculated as in our local RGB-D pose estimation method evaluation, section 9.2.1.1. The errors are only computed if more then one forest attached feature and more then 3 feature points (including the forest attached feature points) are found in our methods. For the EPnP and EPnP RANSAC, the error are computed only if the method returns that the pose was found.

From these, results we can observe that our method has better rotation error then conventional and RANSAC EPnP methods in these case, and comparable results in translation error. Also, we can observe that our method of fully calculating the error of all possible combinations, although slow, generates better accuracy and precision compared to EPnP methods. Another characteristic that we want to point, is that our method has less deviation than conventional methods. 
Figure 58 - Samples of images with and without background used for the evaluation of the methods. First row, with realistic background; second row, without background.

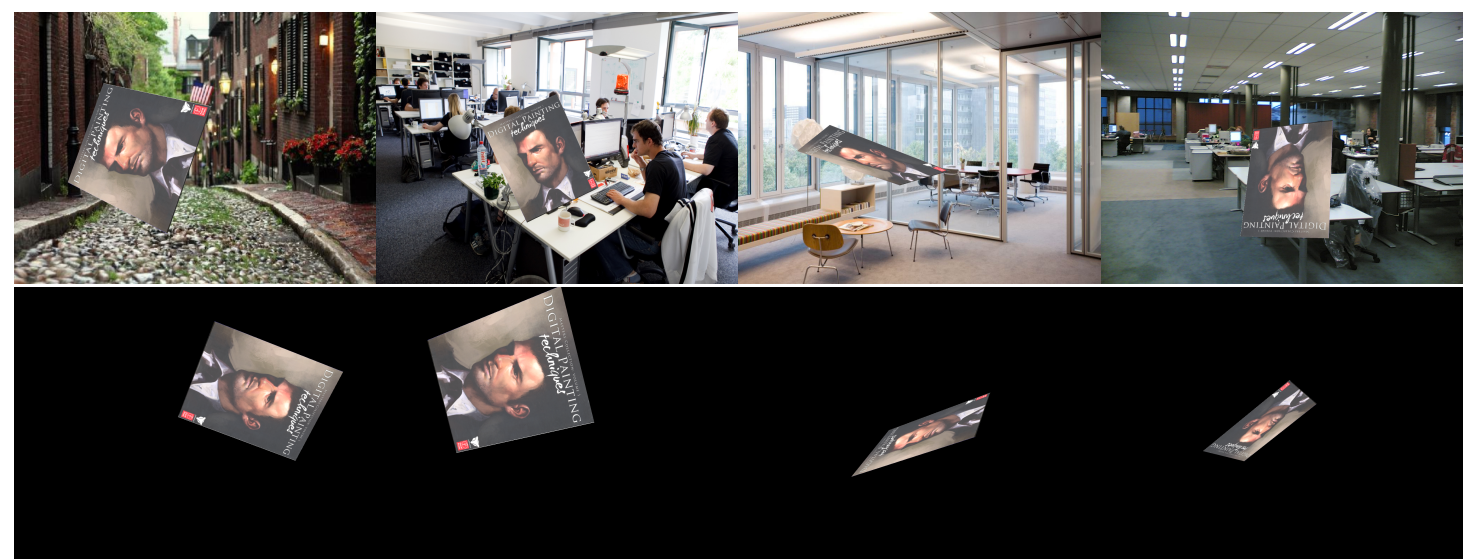

Source: Author.

From the evaluation without background image (first row of 59), we can observe that our method has less accuracy in case there are few feature point matching errors. However, this also means that our method is more robust to this kind of matching errors. This is due to the fact that our method, even using multiple points for verification, needs only 2 points in order to calculate the full pose, unlike solve $\mathrm{P}-n-\mathrm{P}$ methods, that at least need 4 points in order to estimate the full pose.

This effect also can be observed in the case that we variate the total number of feature points to be matched. Showed in figure 60, we also tested our and EPnP methods with several different values of feature points, with fixed number of decision forest attached feature points as same as the last test, as well as using the same images with background.

In image 60e, we show the rotation errors. Note that our method has more rotation error in the beginning, however, that is because our method has higher found rate as shown in image 60b. This happens once our method consider as found when at least one forest attached point is found, however, it also means that less accurate poses are also calculated in the error.

Moreover, once we have fixed the number of feature points with forest attached, our found percentage decreases with the number of feature points increase. Here, matching errors are corrected by the added points, decreasing our error too. However, around 250 to 400 feature points, where the found rate of our and EPnP-RANSAC are similar, we can observe that our accuracy for rotation is better than the conventional EPnP. Finally, from images 60d we can observe that our method perform as good as EPnP RANSAC approach for translation estimation.

In order to evaluate the effect of the number of trees (related to the estimation accuracy), and number of forest attached feature points (related to the accuracy and 
Figure 59 - Box plot of results evaluation of our approach, compared with EPnP and EPnPRANSAC by Lepetit, Moreno-Noguer e Fua (2009) First row: evaluation based on images without background images(flat black background); second row: with realistic images as background. First column: rotation error; second:translation errors.
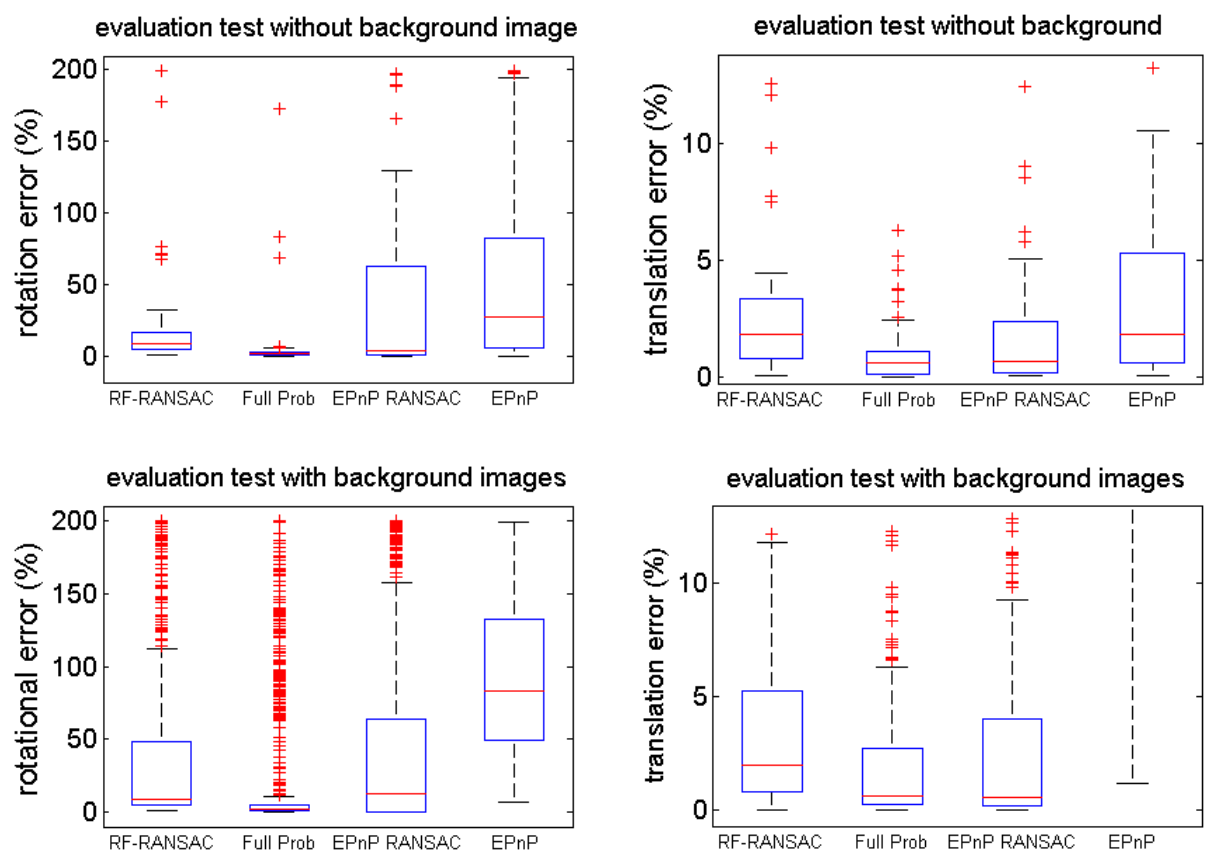

Source: Author.

founding percentage), we proceed a evaluation test across different numbers of tree and forest attached feature point. Results obtained by this test are shown at figure 61 .

From images in $61 \mathrm{~b}$, is possible to observe that with the increase of forest attached feature points, the probability of founding the object pose increases. However, also, the rotation and translation error has increases as shown in 61c, as presented before.

From $61 \mathrm{c}$ and $61 \mathrm{a}$, it is possible to observe that the increase of trees in the forest does not affect the error or the finding probability. This is due to the high probability of regression forest to have some trees with accurate output. This also means that our RF-RANSAC verification of the re-projected point performs better then conventional decision forest, which uses a large number of trees to find the results by combining their results.

\subsubsection{Deformable Object Local Rotation}

More over, by finding the local rotation of each feature point, its information could be used in order to estimate deformable objects poses, that are locally rigid as in (RENDL et al., 2014; TOKUNAGA et al., 2015). We process this local rotation using only the local 
Figure 60 - Error values across different values of feature points. 60e shows the rotation errors; $60 \mathrm{~d}$, the translation error and $60 \mathrm{~b}$, the found poses across the evaluation images.

(a) translation error

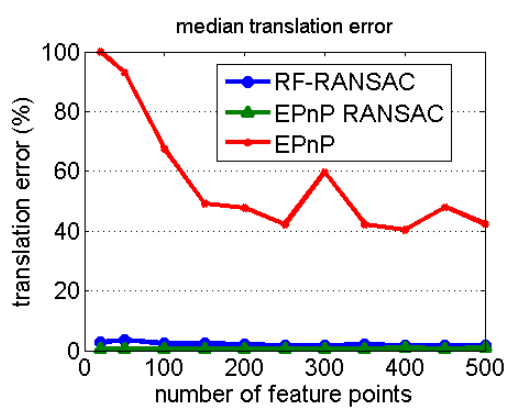

(b) percentage of found poses.

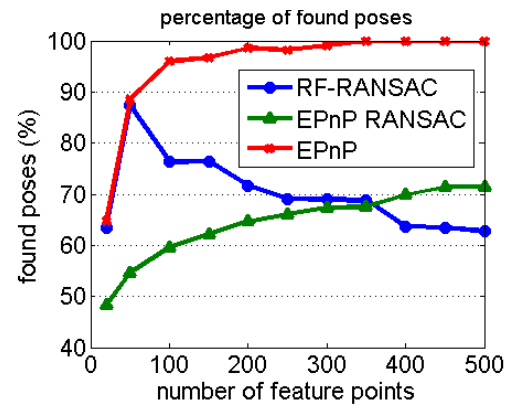

(c) rotation

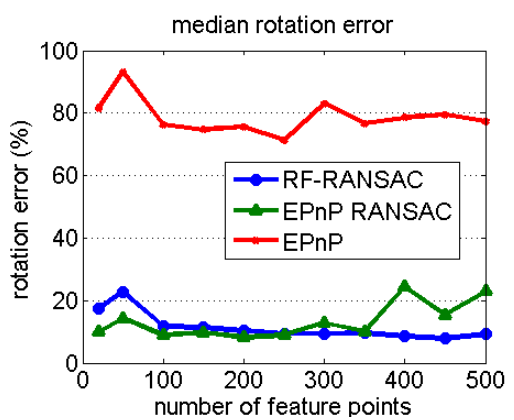

(d) translation error, plot with-(e) rotation error, plot without out $\mathrm{EPnP}$ $\mathrm{EPnP}$
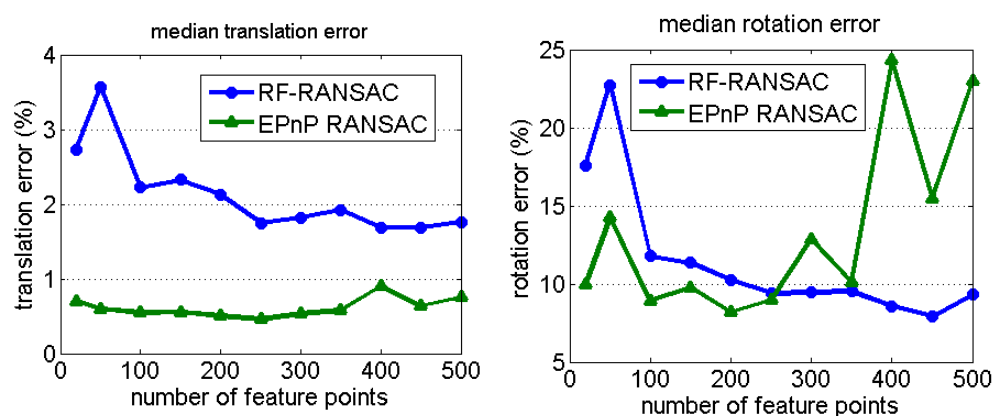

Source: Author.

feature point orientation vector (for the equation 8.6) and conventional decision forest approach, presented in subsection 2.7.1, of the regressed values from all the trees of all the found feature points.

Here, each local rotation obtained by different trees, are converted to quaternions and the mean value is obtained for each found feature point. Then, these rotation are reconverted to find the local rotation. The local rotation results are shown in figure 62 . Each local rotation are represented with a coordinate system rendered over the feature point position in $2 \mathrm{D}$.

\subsection{Result Discussions and Limitation}

In this section we discuss our obtained results; as well as, further proprieties of our approach and methods and their limitations. First, we observe the results of feature point filtering and its impact across this work. As presented in chapter 6 , we had the hypothesis that filtering robust feature points is one factor that could impact the final pose estimation methods across this work, once it can impact on the number of observed 
Figure 61 - Evaluation of translation and rotation errors, and number of founds across all the test set; across different forest trees numbers and different number of forest attached feature points.

(a) translation error

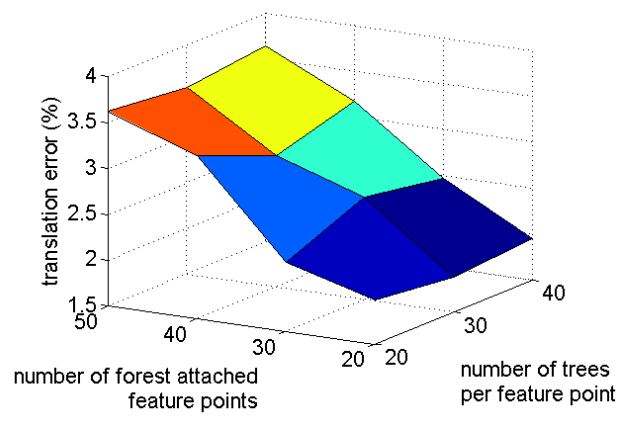

(b) percentage of found poses.

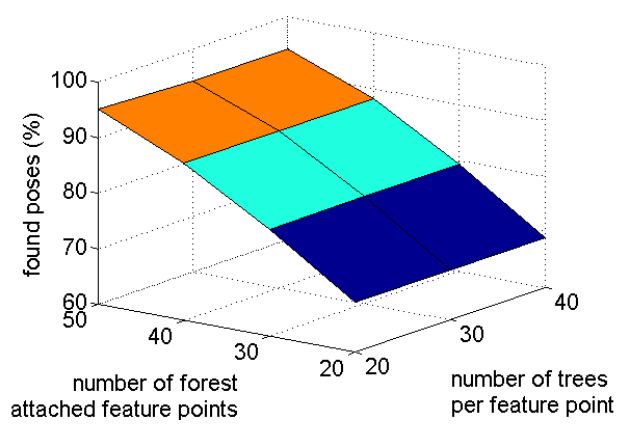

(c) rotation error

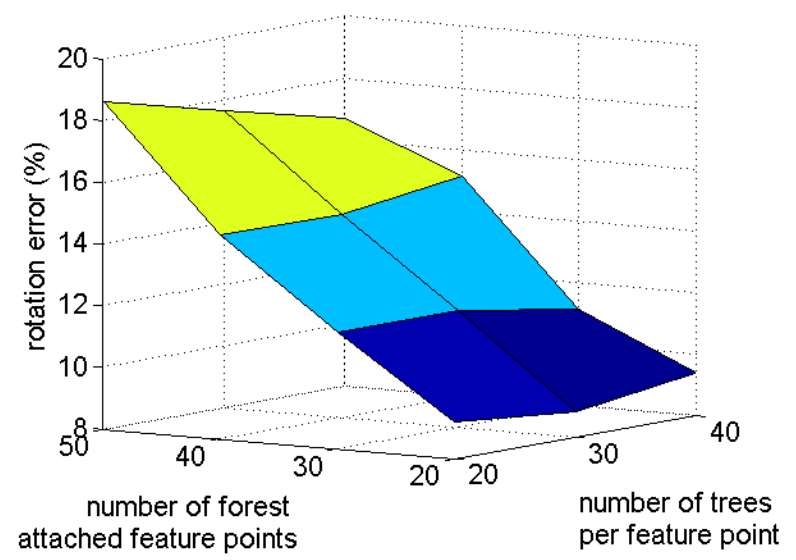

Source: Author.

feature points in different poses.

In order to obtain better pose estimation results, the same feature points should be found in more view positions, increasing the view probability of the real object. This increase is illustrated in figures 35 and 37, where we show our method generates an increase of feature points observed in less orthogonal views of the object.

The impact of the feature points number and quality is also observed in the final pose estimation results. In case our RegressAR pose estimation method based on RGB image, the impact of feature points is illustrated in figure 60, were the probability of finding some pose depends if forest attached features are observed.

However, as shown in figure 60, there is a balance between probability of finding the object pose and its accuracy. Specially in RegressAR method results presented in figure $60 \mathrm{~b}$, where we can observe that there is an increase in the probability of finding some pose with the increase of forest attached feature points, but also its pose accuracy decreases. This indicates that even that more feature points are found, they can return 
Figure 62 - Deformed object local rotation estimated using our regression forest.
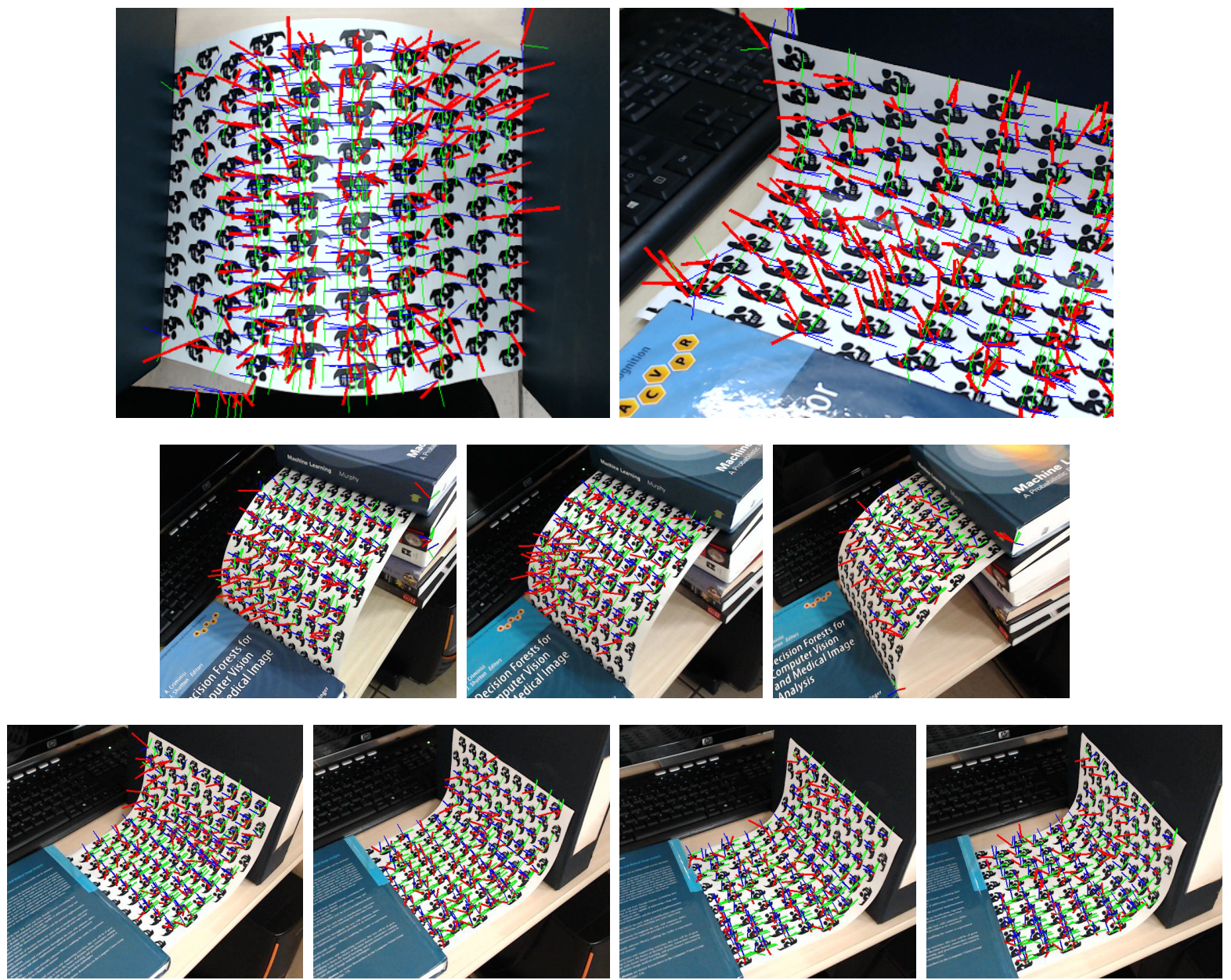

Source: Author.

less accurate regression results, leading to wrong poses.

Besides the robustness of feature points used in the matching, our approaches (in both RGB-D and RGB based pose estimation) also are based in the use of other local information gathered around feature points. In the case of our local pose based on RGB-D information, we add the local normal and feature orientation in order to obtain the local pose; which enable us to achieve unique results, such as presented at sections 9.2.2 and 9.2.3. In case of our RegressAR approach, we add the local texture information and how they deform across views.

However, local information of feature points can lead to different global pose errors, once they lack in global information of the object pose. One common limitation of our local information based approaches is that they are sensitive to local noise. In RGB-D, depth noise around the feature point surface can lead to large rotation errors at the object global pose, as illustrated in figures 47 and 48. This makes our pose results less accurate in presence of small depth map noises. 
This effect can be filtered in rigid object pose detection, once we can use all the observed local poses for global pose estimation. However, in case of deformable and single feature tracking, which directly rely on local poses, these noises lead to less accurate results, once the prior of global pose estimation can not be applied for filtering.

In RegressAR approach, the noises and deformations of local texture also decrease the accuracy of the overall pose estimation. As presented in figure 54, several regression generates poses which globally are inaccurate, even, with the same local texture deformation.

Moreover, any image distortions in the input frame can lead to pose errors in the estimation process. One distortion that can lead to strong errors is the deformation in projection matrix, breaking the assumption $A_{c 1}$. This is caused once the hypothesis of $\psi$ invariance is broken, leading to distortions caused by $\psi$ variation.

This is also true for the images rendered for the training. If these images contain such distortion, the texture distortion between two poses with same $\theta$ and $\phi$ can be different, generating ambiguities inside the training samples, making the training process harder in order to acheive accuracy at the estimation process. Therefore, it is necessary to eliminate distortions such as lens and scale distortions with methods such as proposed by (ZHANG, 2000), in order to achieve pose estimation by local texture distortion.

Our RegressAR approach also has limitation of memory usage. This is because random forest cover imperfections of the regression with several trees, being a memory consuming method. Also, the trees it self also cab be large and deep. In our case, several trees has more than 10000 nodes each. Added to this, we use normally 50 trees per each forest attached feature point, and 20 features; adding up to 1000 trees per object. Increasing the number of forest attached feature points, can increase the probability of finding the pose and its accuracy, however, it also increase the memory usage.

Finally, the accuracy of each tree attached to feature point is another issue in the object pose estimation. As presented in figure 54a, similarities in the features samples creates several trees with wrong regression values. These errors are filtered by adding global observation of the object by RF-RANSC method, section 8.4, as done in RGB-D pose estimation. However, this also makes hard the usage in deformable objects. In theory, if per tree per feature object pose accuracy has an increase, it is possible to achieve full local rotation, enabling similar results as in RGB-D object poses.

We had listed, until this point, limitations of our local information approach, both in RGB and RGB-D capture setup. On the other hand this local approach has several advantages across different conditions, even when compared to state-of-art methods. In RGB-D local pose based method, which is calculated by local surface normal and orientation, the local pose enables the estimation of deformable objects poses, as shown 
in 9.2.3, and by single feature point tracking, in 9.2.2; both, results not achieved by conventional pose estimation methods based on RGB-D cameras.

Also, we shown at 50 that, despite the limitation of pose error created by depth map noises, we can achieve better and faster results in RANSAC based methods, when compared to conventional state-of-art methods. Our method shows to be more robust across RANSAC parameters settings, and faster across capture noises with fixed RANSAC setup. Indicating that our method needs less fine tunes of the algorithm for different setups. This could be because of the increase in information quantity, gathered from feature point local information, for the same capture conditions.

In RGB capture conditions, our Regress AR approach performs better when compared to $\mathrm{EPnP}$ methods in realistic conditions, despite the fragility across distortions. Even when compared to EPnP-RANSAC approach, which is more robust in presence of outliers, it shows to be better in both accuracy and precision for some of the tested conditions.

Moreover, our full probability set calculation method, presented in equation 8.18 at section 8.4, although slow, shows to be more precise and accurate when compared to other approaches, as shown in figure 59. This not only shows the potential of our approach, but also illustrates that RF-RANSAC method tries to find, across all the possibility set, some suitable results in some suitable time. And the best result in this full probability set is the one returned by the full probability set calculation method, which can outperform stat-of-art methods. Making it, a searching problem across the large set of poses.

Although, both RGB and RGB-D results are not fully explored in real-time conditions in this work, both approaches are based on methods that conventionally can generate fast results for real-time systems, such as RANSAC (FISCHLER; BOLLES, 1981) and regression forests (CRIMINISI; SHOTTON, 2013). Those decisions where made in order to be able to explore our approaches in real-time AR applications as future works.

Moreover, both RGB-D and RGB based approaches could easily explore parallel processing methods, once both consider the local information, which are independent to each other. In special, our RGB based RegressAR approach is based on decision forests, which has several results showing its potential for real-time applications when processed in parallel computing (SHOTTON et al., 2011; SHOTTON et al., 2013; CRIMINISI; SHOTTON, 2013).

In Special, Sharp (2008) presents that some computer vision tasks could have almost an increase of computational speed of 100 times, when compared to conventional CPU based processing. This strongly indicates that our RegressAR method also could benefit from the parallel processing, reaching real-time applications performances. Or even more, our full probability calculation of equation (8.18), which presents higher accuracy 
and precision compared to conventional methods as shown in images 59, could be processed in real-time.

\subsection{Chapter Conclusion}

In this chapter we presented our obtained results of the entire work; as well as, the discussions around the results and the limitations of our approaches. From the results, we show that our approaches of gathering local information around each found feature point can outperform state-of-art methods presented in literature. Moreover, our results opens entire new possibilities for future research, due its novel nature.

Also, these results proof our research hypothesis, presented in chapter 1. Showing that local information can be used and improve estimation of different objects poses. Thus, accomplishing our objective, section 1.2. In the next chapter, we present further discussions around our work, such as future direction, direct or indirect relationship with other ideas and methods, and specially, its usage for AR and interaction methods. 


\section{Further Discussion}

As presented and discussed in chapter 9, our approaches based on gathering local information around feature points for pose estimation, open several future researches due to its novel characteristic. In this chapter we focus on further discussions around our work, such as the local information based pose estimation approach characteristics, as well as, their usage in different scenarios and applications. Thus, bringing to spotlight, hints of its research potential across several views.

\subsection{Human Perception, Feature Point Local Information and Lo- cal Pose Based Approach}

The first topic that we discuss here, is about the local information based approaches that we have taken for the pose estimation. Local information for several works in the literature (LEPETIT; LAGGER; FUA, 2005; LEPETIT; MORENO-NOGUER; FUA, 2009; HENRY et al., 2012) are used to find the interest points and its matching (BAY et al., 2008) with another reference. For this purpose, the texture of the object is analysed. However, these information are simplified as points in space in order to calculate the object pose, as pointed in chapter 3 . Although it is true that this simplification is computationally sufficient for the pose estimation, more information can be gathered in order to obtain the object pose.

On the other hand, humans use other visual clues in order to estimate an object pose in the space such as shades, outline edges or changes in gradient of known texture as polka dots (TODD, 2004). And, from these clues, humans can train pose estimation of objects by piece-wise or by the entire object (EDELMAN; BULTHOFF, 1992). Our approach for pose estimation relies on these human perception in order to process the pose of objects.

We include observation of local feature points, and then, estimate the entire object pose. Our RGB-D based pose estimation is similar to finding the object pose by finding the object surface geometry, and by these information, the entire object pose. On the other hand, our RegressAR approach is more directly similar to the process of how humans can perceive shapes by polka dots (TODD, 2004). However, instead of using dots deformation, our approach uses small image patches. Which are estimated by matching trained deformations with the observed texture deformation. In these way, we show that local information can be gathered in order to proceed the pose estimation.

Also, once local information can be independent of the entire global pose, they 
make possible the pose estimation of deformable objects. Moreover it is also possible to obtain the pose of the object, when a small amount (or even from a single) of feature points are observed. Thus, also illustrating the richness that local pose can bring.

However, as pointed in section 9.4, local poses has the disadvantage of being affected by local noise, and these pose errors, once lack in global information, can generate large errors in the final object pose. This shows that accuracy of the local pose is an issue for the pose estimation. Still, local information can definitely bring additional information for pose estimation, as hypothesized in chapter 1 and proofed by our results at chapter 9 .

\subsection{Locally Planar Assumption $A_{o 2}$ Aside}

In order to achieve our pose estimation, we have taken different assumptions long this work. Some are strong assumptions about the object; in particular the assumption $A_{o 2}$, which considers that the surface around each found feature point is planar and rigid. This assumption is taken in order to simplify the object surface and the generation of synthesized data for the feature points extraction, chapter 6 ; calculation and extraction of surface geometrical information, sections 7.1; as well as, the training of the surface texture distortions, section 8.1 .

On the other hand, as presented in section 9.2.1, our methods can be applied even in objects with surfaces that break this assumption, such as cylindrical ones. Thus, showing that this assumption can be discarded for other types of objects, which has non-planar locally rigid surface. However, in order to discard this assumption, several changes in our approaches and methods needs to be applied.

First, in the robust feature points extraction process, chapter 6, 3D mesh models of the object need to be used in the rendering, instead of planar representation of the object surface, as in (LEPETIT; LAGGER; FUA, 2005). This is done in order to analyse the robustness of feature points, even when their surface are not planar. It is also possible to analyse effect of self-occlusion caused by the object surface.

With this change, it is possible to exclude feature points which, although robust across views as planar feature point, are often occluded by the shape around, such as feature points placed at the surface concave parts. Thus, making the extraction of feature points that are more robust across the object pose changes.

In the RGB-D capturing setup, we also need to change our pose estimation methods, section 7.2, in order to discard the assumption $A_{o 2}$. This assumption is specially important at equation (7.9) and the rotation matrix calculation (7.10) and (7.11). Where, the normal of the feature point is calculated by the cross product of two vectors in the surface, which is true for planar surfaces. 
However, this is not true for non-planar surfaces. In order to obtain the surface normal or pose, we could use more observations of the feature point surrounding surface. This could be done by using simply using more vectors for the cross product, sampling different parts of the surface; or using methods such as ICP (NEWCOMBE et al., 2011) with the color and geometrical information. Thus, achieving the alignment of the small local surfaces, and its transformation.

In case of the RGB capturing setup, our RegressAR approach need to be changed by its training step. Initially, the rendering of the synthesized data, section 8.1, needs to use a 3D model of the object. Also, for each found feature, instead of using the direct camera $\theta, \phi$ and $\psi$ for the training, we need to convert those values to relative values of the local surface coordinate.

On the other hand, despite the similarities problem, directly using $\theta, \phi$ and $\psi$ values of the camera for the training also could be tested. With this, we could achieve training without the need of the unfolding model. Also, if the data is captured directly from the real object, instead of rendering it, we could achieve the regression without the need of the 3D mdoel and its 3D positions, as in (MURASE; NAYAR, 1993).

In both RGB-D and RGB capturing scenarios, relaxing the assumption $A_{o 2}$ could bring more accurate results and poses of more types of object. However, using 3D models, also could decrease the number of observed feature points, or create similarities between views; leading to a decreasing of the pose accuracy. Thus, more studies need to be proceed in order to evaluate trade off created by these changes.

\subsection{Light Field and RegressAR}

In our RegressAR approach, chapter 8, we gather the surface texture appearance changes in several poses, in order to train the object poses. This idea of extracting the light intensity reflected by the object in several poses is similar to the idea of Light Field (KIM et al., 2013; WANNER; GOLDLUECKE, 2012). Light field can be defined as the 4 dimensional information of the capture light, containing not only the 2D image information, but also, the angular information of the light rays in each pixel of the 2D image. From these information, it is possible to extract spacial information (WANNER; GOLDLUECKE, 2012).

This $4 \mathrm{D}$ information of the light field is conventionally represented by two parallel planes, such as illustrated in figure 63a. In cases where a complete [360, 180] degrees of representation is needed, it can be represented by a box made by the parallel planes; or by a sphere, with the entering angle and escaping angle of the ray, illustrated in figure $63 \mathrm{~b}$.

In our case, the RegressAR could be represented as a spherical light field of the 
Figure 63 - Light field representations.

(a) Two planes representations.

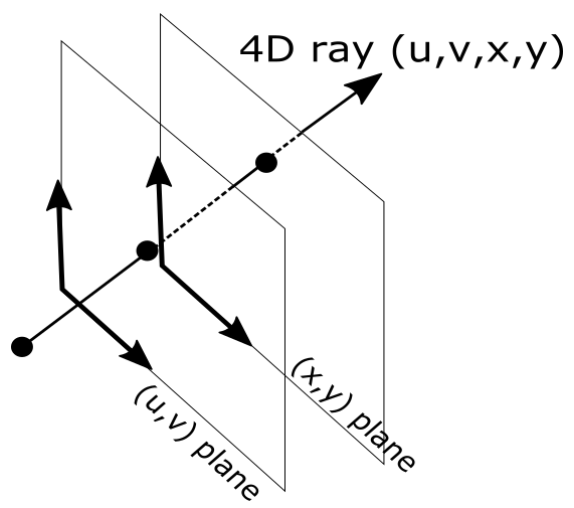

(b) Spherical representations.

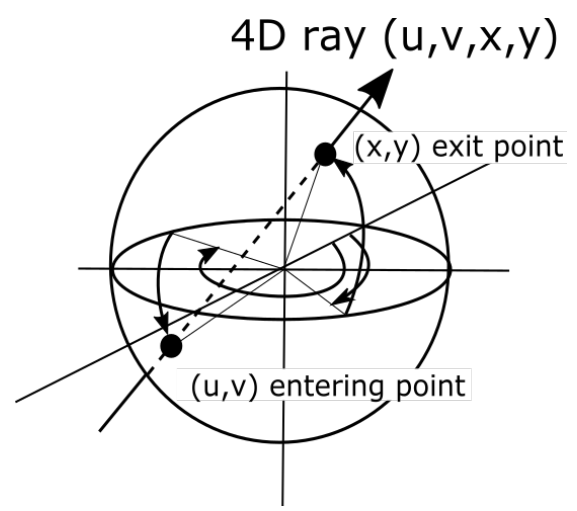

Source: Author.

small image patch used as feature values at the training, in section 8.1. Note that our case is also a special case, where the center of each image patch always points toward the center of the light field sphere. This is due that our feature point has always the same central position, created by the matching.

Now, with this light field representation, our pose estimation problem turns to be a search problem for the best possible ray set positions $(2 \mathrm{D})$ and angles $(+2 \mathrm{D})$ to the camera position, over the 4D domain. This approach can brings several advantages. First, once the ray angles also needs to be estimated, it brings us the $Z$ position of object to the camera. Also, analyzing the gradients or tensors in all the 4D domain, such as done in (WANNER; GOLDLUECKE, 2012), we also can obtain the changing profile of the surface texture over pose changes, giving us more prior knowledge about the object over time.

Finally, if a complete light field capturing is considered, we our regression problems be extended to a higher dimensional problem. Where, instead of having a 2D image patch as learning features, we could have a 4D image patches for training. This could bring better regression results, or even the directly training the depth of each patch. However, this also makes the regression problem more complex; which also could bring other problems or noises to our results.

\subsection{RF-RANSAC: Other Usage and Its Performance}

In order to filter and obtain the global pose in our RegressAR approach, we proposed the RF-RANSAC methods, section 8.4, which uses prior information of the entire object, together with decision forest (CRIMINISI; SHOTTON, 2013) and RANSAC method (FISCHLER; BOLLES, 1981). However, this method could also be applied in several other cases, where other secondary information are available. 
For example, in object classification problems, such as (GU; REN, 2010; BOSCH; ZISSERMAN; MUOZ, 2007; LIN et al., 2015), information of the relationship of classified objects in the same scene can be added to each classification tree, filtering the results. As an example, for any scene image, object classification could be filtered by RF-RANSAC if we add the probability of finding these object together (Such as, if a "car" is found, the probability of some other object be a "gas pump station" is higher than it be a "music box" or a "refrigerator"). Other then these information, color information of other parts or other feature can be included in order to filter the result with our RF-RANSAC.

Also, in tracking problems (ISARD; BLAKE, 1998; KALAL; MATAS; MIKOLAJCZYK, 2010), the temporal prior can be used. In these cases, the prior in equation (8.13) can use the prediction model of the object, and the decision forest results can be used as observations, such used in Kalman Filtering (KALMAN, 1960). Also, the confiability of each tree can be updated with this temporal information. Making it similar to a dynamic version of Boosting methods (MURPHY, 2012), where the probability of each tree are updated dynamically thought the capturing.

Moreover, once our method is based on decision forest approach, it shares its characteristics of being able to explore parallelism in order to boost its computational speed as presented in (SHOTTON et al., 2011; SHOTTON et al., 2013; BRACHMANN et al., 2014). This can be done by making all the decision trees of all observations in parallel by implementing it in parallel process units such as GPU (CRIMINISI; SHOTTON, 2013). Also, in our case, the prior information of the point distances could be processed in parallel. By this change, we could achieve fast processing, or even the calculation of full set probability in equation (8.18) of section 8.4 in real-time.

Note that our RF-RANSAC is similar to Boosting methods (MURPHY, 2012). In these methods, each tree is used as a weak learner, and the relation of their results are weighted in order to estimate the final result. However, also note that our method differs from these methods, which calculate the trees weights based only in the training samples. In contrast, our method uses external data from the observation in order to obtain the final result. This characteristic makes the RF-RANSAC more consistent with the observed data.

\subsection{Local Pose And Other Methods}

This works is centred in the idea of gathering local information of feature points in order to extract the local pose, and then, calculate the final results of the object pose. Here, we filter the local poses to the global pose using methods such as based on RANSAC approach, as shown in section 7.3.1 and section 8.4; or by calculating the deformable object pose by the nearest found pose, section 7.3.2. 
However, our calculated local poses are independent from each other, once only local information is gathered. This propriety makes possible the local pose usage with several other methods in literature. Unlike other methods, that process the same feature point position data in different ways (LEPETIT; MORENO-NOGUER; FUA, 2009; TORKI; ELGAMMAL, 2011; HENRY et al., 2012), our local pose based approaches gather further information from near neighbour of the feature points. Information which are not conventionally used in several global information based methods. Making its usage in combination with other high abstraction methods easier.

In this section, we focus on three different types of methods, which can be combined with local poses, and are conventionally addressed with pose estimation in AR systems or in interaction methods. The object classification, pose tracking and deformable pose estimation.

\subsubsection{Classification}

When several object needs to be found in the same scene, classification methods needs to be applies (SOCHER et al., 2012). Such methods classify which objects are being observed, based in several information. In the context of this work, the classification can either increase the pose estimation accuracy, by filtering positive errors matched filters, or be attached after our approach, being able to use also pose and geometrical information for in the classification itself.

If the classification is proceed before our local pose estimation, by methods such as (SOCHER et al., 2012), its results can be used in order to increase the accuracy and precision of our approach. This is done by filtering wrong matches of feature points. These false positives results, not only can return wrong results, but also contaminate the over all global pose. Our RGB-D and RegressAR approaches accuracy could be increased once those outlier points in the global pose verification in the RANSAC method are pulled out. The deformable pose estimation accuracy also could be increased in RGB-D cases.

On the other hand, if the classification is proceed after our local pose estimation, our local poses can be used as additional geometrical information of the object in the classification. These geometrical information can be added to methods such as (GUPTA et al., 2014). In this work, geometrical information is used with deep convolutional neural networks $(\mathrm{CNN})$ in order to process the classification. Replacing our local information instead of the geometrical data, may lead in a increase of the classification results.

This same approach could also be applied in works such as (LAI et al., 2011), (SOCHER et al., 2012), and other works, which used geometrical information in combination with RGB data in order to process the classification. Thus showing that our local pose idea can be extended or extends several works of classification. 


\subsubsection{Tracking}

Tracking and filtering task is the next important step in AR. Tracking can be defined as finding the object pose across sequential time observations, by also filtering out noises of the pose estimation process. This temporal information generates more precise and accurate registration of the virtual information, which can directly affect the user perception in augmented reality.

The most straight forward way, in order to perform this tracking, can be by applying Kalman filters (KALMAN, 1960) in our final global pose. Using Kalman filters, the tracking problem can be defined as multiple gaussian prior and posterior probabilities (or errors) of the pose, and proceed the filtering by calculating the Kalman filter gain between the last state prediction and the new observation.

We can directly set as the Kalman filter parameters our 6D global pose. However, more can be done in order to enhance our results, by also filtering parameters such as the orientation vector in RGB-D local pose of the feature points, presented at section 7.2 , or the matching features of the features points, in order to enhance the feature matching process itself.

This Kalman filter approach also can be combined with the single feature point pose estimation, presented at section 7.2. By applying the Kalman filter in the pose and feature point itself, we can filter problems of non observation of the feature point, or pose noises created by RGB-D noises.

However, once we can obtain several observations of the global object pose, by calculating the local poses, we also can apply a particle filtering (ISARD; BLAKE, 1998) approach in order to track the object over time. In particle filtering, several observations with different probabilities and prediction models are used in order to find the final object pose, and refined across time, by filtering particles that has greater probabilities when compared with the final pose observation.

In our RGB-D pose estimation, the local poses of different feature points can be used as different particles, and for each new frame observed, feature points with higher pose accuracy can be filtered. Also, new feature points can be added in the filtering, other than the pre-processed ones, section 7.2 , by capturing in real-time the feature points $3 \mathrm{D}$ information.

In the Regress AR approach, we can use the particle filtering idea, by combining the Gaussian prior probability in the RF-RANSAC method, section 8.4. Each decision tree of each feature points can be considered as an observation of the object final pose, and the time prior knowledge can be calculated separately as a Gaussian distribution, which is updates by the decision trees. Trees or feature points with less accuracy can be filtered out, which could make the final pose more accurate with more computational performance, 
by using less inaccurate decision trees.

Finally, methods such as PN-Learning (KALAL; MATAS; MIKOLAJCZYK, 2010) can also be combined with our local pose in order to perform the tracking task. Each feature point and its 3D information can be re-trained on real-time, based on the Positive(P) and Negative(N) learning approach presented in the Kalal paper.

These examples, shows how tracking methods can be applied with our local poses idea, in order to enhance the final augmented reality results. Once we focus on local pose detection, several tracking approaches can be combined with our results.

\subsubsection{Deformable Objects Pose}

In order to estimate the object pose deforming across time, we have simplified this complex problem, into a more simple problem of finding the nearest local pose, obtaining a rough pose of the deformed object. However, we also can apply more complex deformation models in order to fully estimate the deformation of some object.

One model used in literature, in order to estimate deformations across time, is the snakes model (XU; PRINCE, 1998). Works such as (BELLMORE; PTUCHA; SAVAKIS, 2013; SCHULMAN et al., 2013), are based in this model, where, an energy minimization between the observed gradient vector flow $(\mathrm{GVF})$ and proprieties of the resulting shape are processed in several iterations in order to find the final deformation of the shape.

In case of our work, instead of calculating GVF in 3D space based on the spatial information, a GVF in 6D space, based on the geometrical information and local poses, could be applied. Moreover, feature values of the feature points also could be used in GVF in order to add appearance similarities information in the pose estimation.

Also, the particle filtering approach presented at section 10.5.2 could be applied in order to fully estimate the object deformation. Based on a similar approach presented by Isard e Blake (1998), we could track the 3D shape of the object surface by using different particles spread across the object surface.

Another way to obtain the deformation of an object, could be by apllying a energy minimization approch with the locally rigid assumption as in (LI; SUMNER; PAULY, 2008; LI et al., 2009). Those works use this approach to estimate deformation of object such as human skin or cloth deformation, based on depth maps. Same approach could be done with our local poses in order to also estimate the object full deformation, also using the local pose inside the minimization method.

Moreover, machine learning methods also could be applied for this deformation estimation problem. Rendl et al. (2014) achieve the deformation estimation by using piezoeletric sensors placed across a thin-film surface, and training their responses across 
different deformations with k-Nearest Neighbor (MURPHY, 2012) method. This process approach could be also combined with our local poses, where these information could be trained in place of the piezoeletric sensors.

\subsection{More Objects and Information for AR}

In this works, we have focused in two types of objects to be used as markers in AR systems, the rigid objects and deformable objects. However, more unconventional types of objects also could be used as markers in AR system, in order to create novel registration between virtual and real worlds. Moreover, knowing the marker information can give us more information from the capture scene, than the object pose. In this section we present different possibilities of new types of markers and or new usage of the markers in order to estimate different information for augmented reality.

One example of object, are the piecewise-rigid objects, which could be used as markers for AR systems. Pauwels, Rubio e Ros (2014), present a tracking approach for piecewise-rigid articulated objects, such as folding objects. With this kind of tracking method, objects such as books or cards, which can contain strong meaning embed in reality, could be tracked.

Our local pose idea could be directly applied in this piecewise-rigid object tracking. Once each piece of the object is rigid, the same approach used in our RGB-D pose estimation could be used in order to find the pose of these pieces. This, again, is possible once our approach is based on local pose of the feature points. Moreover, once our local pose approach can retrieve the pose/rotation from a single feature point, even poses of pieces that have heavy occlusion, with only a single feature point visible, can be estimated; unlike the original method. Figure 64 illustrates this idea.

Scene reconstruction itself could also be collected in order to make the AR perception richer. These environment geometrical information could be estimated by methods such as SLAM (DAVISON, 2003) or PTAM (KLEIN; MURRAY, 2007). With these information, it is possible to extends the AR effects to outside of the estimated object pose, for example, making possible that virtual characters walk around the scene; or casting shadows of the virtual objects into the real world.

Environment location and mapping methods, such as presented above, could be used by itself for AR systems. However, they lack in knowledge of what is observed. And, often, the information of what or where is to be augmented with virtual information is important in AR systems. This makes usage of known objects markers in augmented reality still an important aspect in AR.

Despite that, our local pose based approaches could be used here to increase results 
Figure 64 - Articulated objects pose estimation using our local poses. Note that in cases that few feature points are visible because of occlusions, conventional point based methods could not be used, figure 64b. Unlike our local poses cases, figure $64 \mathrm{c}$.

(b) pose estimation by conven-

(a) observation of the object through two time steps.
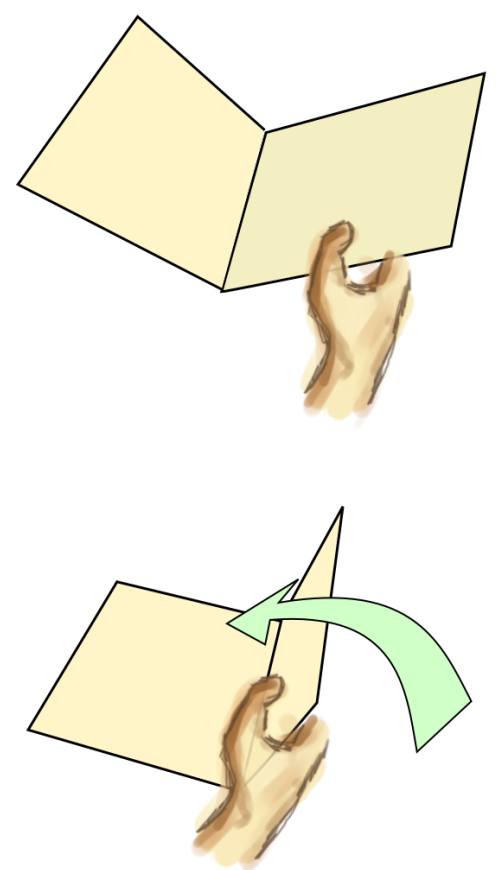
tional methods based on tri-(c) pose estimation based on angles.
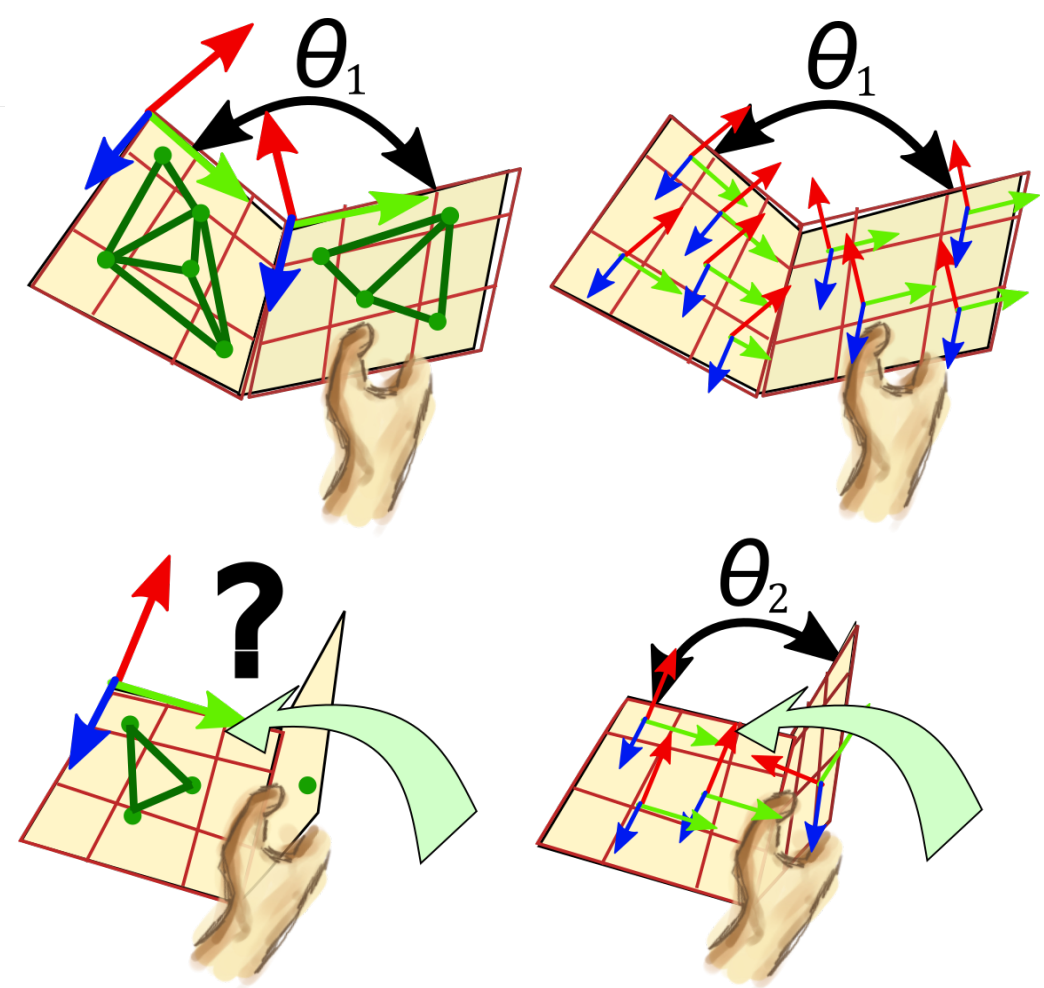

Source: Author.

of camera estimation. Each feature point added in the reconstruction process could also contain the local information, in order to estimate the entire scene pose with a single feature point. Also, the appearance changes of each feature in different poses could be trained as in our RegressAR approach, in parallel to the reconstruction. Thus enabling the scene pose estimation with machine learning approaches.

Moreover, several works in SLAM (DAVISON, 2003) needs to have a camera motion model of first frames, in order to extract the 3D information of several 3D points. Instead of using these motion models, the rigid objects used in this work could be used in order to retrieve the motion of camera in the initial frames.

On the other hand, once the marker information are known, other information, other than geometrical information of the scene also could be estimated. Jachnik, Newcombe e Davison (2012) presents a methods to estimate light sources and environment map based 
on the specular refraction of the object surface and light field information. Similar to their work, knowing the material characteristics of the object can enable us to retrieve information of the scene, such as light source and environment map.

Moreover, information related to the capturing system itself can be retrieved from the known markers. Works such as presented by Yuan et al. (2007) perform image blur correction base on noisy images. However, once a known object is captured, the image deformation of this object can gives us how the motion blur occurs, making possible the blur correction. Similar approach could also be applied for camera noise (LIU et al., 2008), white balance (HSU et al., 2008) and exposure (CANDOCIA; MANDARINO, 2005) estimation.

These camera parameters and environment information are needed specially for rendering the virtual object in same conditions of the real world, making it more blended with the captured scene, as presented by (JACHNIK; NEWCOMBE; DAVISON, 2012), (SUGANO; KATO; TACHIBANA, 2003) and (KŕIVáNEK; FERWERDA; BALA, 2010). Figure 65 illustrates the effects of these parameters in AR systems.

Figure 65 - Camera parameters estimation impact in AR. Using the table as marker.

(b) Correct illumination esti-

(a) original image

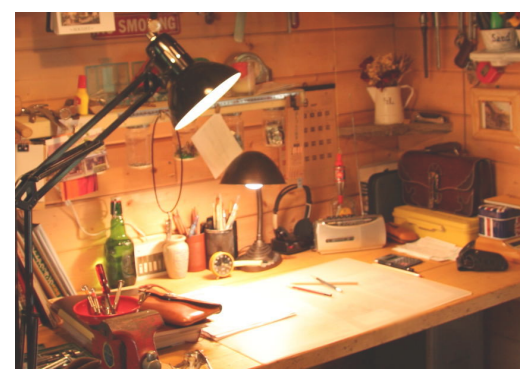

(d) wrong exposure estimation.

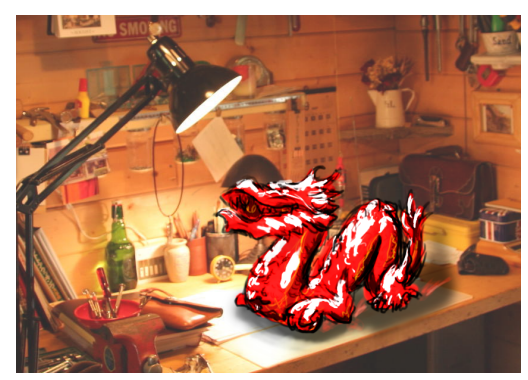

mation and rendering, and (c) wrong illumination, no correct shadow.

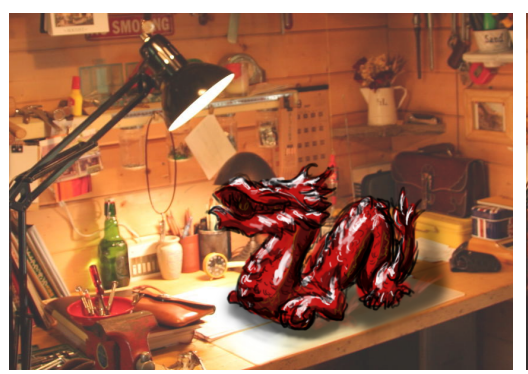

(e) correct motion blur estimation and rendering

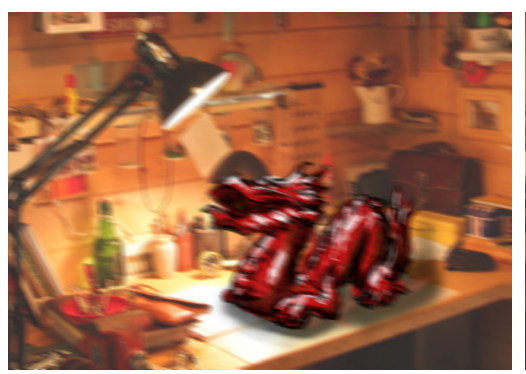

Source: Author. (f) no motion blur estimation.
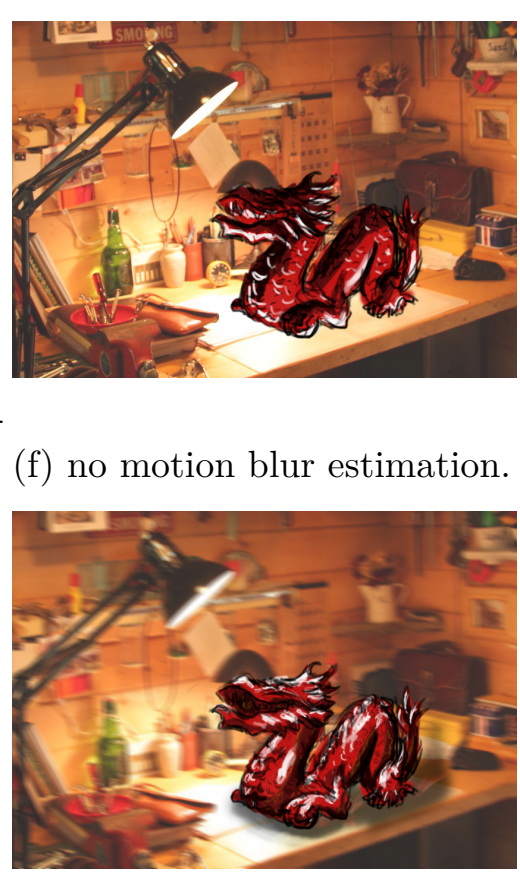


\subsection{Markers for Interaction}

Other than used in registration, the objects used as markers in AR, can also enable different interaction of the user with the virtual information. In this section we explore possibilities of creating novel interaction methods based on results obtained from our approaches and other works in literature.

Tangible interactions, (ISHII; ULLMER, 1997), uses real objects as interface for interact with virtual information. Such interactions has some advantages such as enabling haptic feedback for the interaction. And when combined with AR, which requires spatial registration, interactions with 6 degrees-of-freedom can be done.

Also, several works in tangible user interface, such as (YAMASHITA et al., 2007; ULLMER et al., 2008; SCHIETTECATTE; VANDERDONCKT, 2008; PEDERSEN; HORNBAEK, 2009; MAZALEK; NITSCHE, 2007; BAUDISCH; BECKER; RUDECK, 2010), show that novel tangible interactions can be created based on the object classification and its pose. Pedersen e Hornbaek (2009), Schiettecatte e Vanderdonckt (2008) and Baudisch, Becker e Rudeck (2010) also show interactions that not only rely on the objects pose, but also depends on the spatial relationship of different objects.

Specially, Baudisch, Becker e Rudeck (2010) explores also the possibilities of stacking the real objects as an interaction model. Therefore, changing the pose interpretation according the relationship between objects. Such objects poses could be directly estimated using our approaches.

Different types of objects also can lead to different solutions in novel interactions. Steimle, Jordt e Maes (2013) show interactions that can be achieved by using a flexible papers and projection mapping. Different deformations, such as concave, convex or twisting deformation, are used in order to change the virtual information that are projected over the paper.

Rendl et al. (2014) also detect the pose of deformable transparent sheet, using several sensors spread over the surface. Once the deformable objects is transparent, it also enable overlaying the sheet to another surface and enable interactions based on the sheet flipping, changing the content displayed only in the flipped parts.

In our first figure 1 of this work, in chapter 1, we have illustrated possible interactions for AR based on our results. First, using a rigid object position, then deformable object as rendering and input method for games, and finally, visual extension of real objects, making the real book logo in $3 \mathrm{D}$.

Moreover, the real objects used here, instead of being passive, could also actively collect more information or change its nature according the virtual conditions. Poupyrev, Newton-Dunn e Bau (2006) presents the interaction with D20 die like display, which can 
senses touch, and changes its content according the situation.

Brockmeyer, Poupyrev e Hudson (2013), also show an approach of interaction based on curved display surface that can sense touch. Once these displays could take different shapes, real objects with special meaning, such as mascot character, could be created for interaction, as presented in the work.

Also, more simple fabrication processes could be applied in order to create objects that gather information or changes its appearances. Lo e Paulos (2014) presents a circuit prototyping based on conductive ink and polystyrene sheet that shrinks with heat. Wiethoff et al. (2012) also present prototyping of tangible interface prototyping based on conductive ink.

Olberding, Wessely e Steimle (2014) presents a fabrication approach of interactive displays based on printing the circuit over surfaces, such as paper or plastic sheets. With this approach, several active objects with different shape and deformation could be created as marker for AR systems.

Another approach for creation of novel interaction methods is by applying objects that has actuation (POUPYREV; NASHIDA; OKABE, 2007), which can change its shape (NIIYAMA et al., 2015), move around the scene (PEDERSEN; HORNBæK, 2011), move other objects (FOLLMER et al., 2013) or change the environment conditions (HEUN; HOBIN; MAES, 2013). Attaching such freedom for physical objects to interact with the real environment are explored in literature.

Ishii et al. (2012) propose the concept of Radical Atoms, where virtual information can affect the real world conditions, creating a feedback system of the virtual information back to the real world. Unlike conventional AR systems, where virtual information is just visualized over the real world, Radical Atoms proposes the concept that virtual information can directly change the real world. This idea can also be combined with the idea of Internet of Things (IoT) (HOLLER et al., 2014), where real objects could be controlled or accessed by internet, making possible the control of the environment by virtual information, such as done by (HEUN; HOBIN; MAES, 2013).

In this thought line, real objects used as markers in AR could also be actuated and changes its information according to virtual interaction of the user, where virtual objects could collide and move real objects; or user interaction could change the object shape. In figure 67 we present a concept of interaction between actuated objects and AR.

Finally, even the simple view position change can be used as interaction tool, as presented in (DOSTAL; KRISTENSSON; QUIGLEY, 2013). Real markers could changes its content to be augmented, based on the user view position. In figure 67 we present an interaction method for single and multiple users, which explores the simple view position as tool for interaction. Here, the entire user experience can be changed by only applying 
Figure 66 - Usage of actuated objects as markers. Real robot as marker to AR, where the marker itself can sense and actuate over the real world.

(a) real observation.

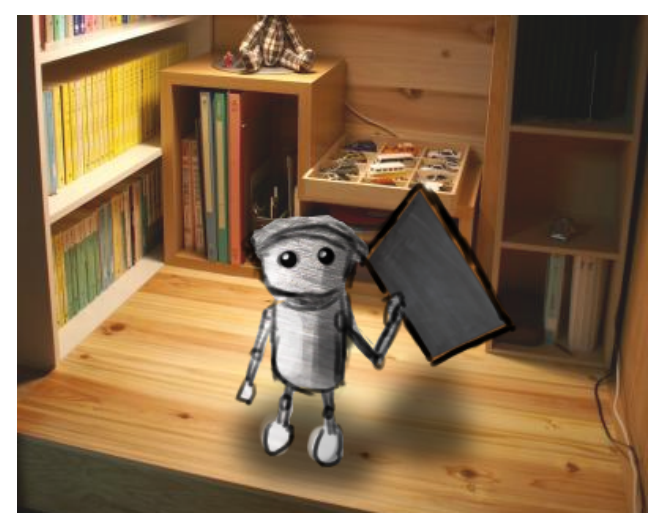

(b) augmented scene with virtual information.

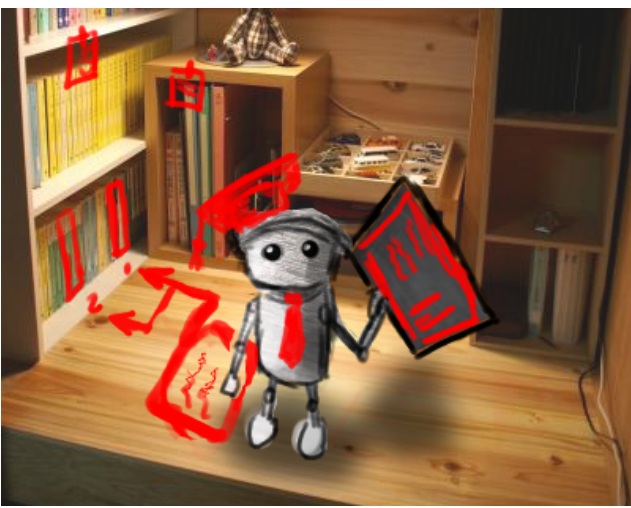

Source: Author.

this positional information.

Figure 67 - View position dependent AR. Display table used as marker. In the same environment, different virtual information (drawn in green) can be rendered according to users positions. Adapting the virtual content to each user.

(a) User A view.

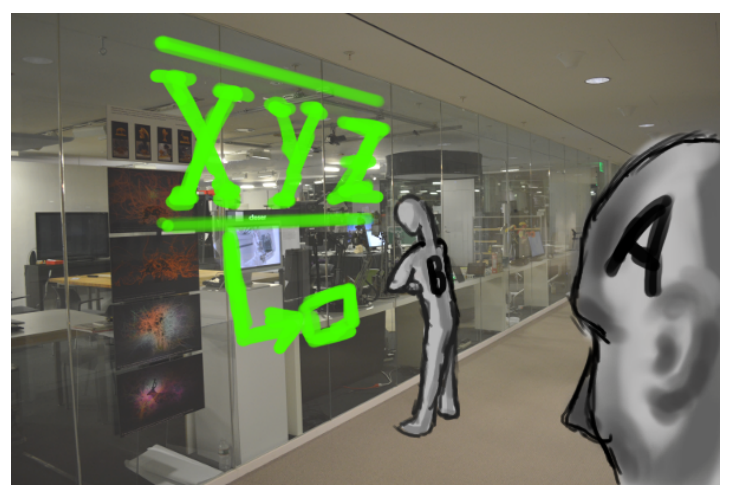

Source: Author. (b) User B view.

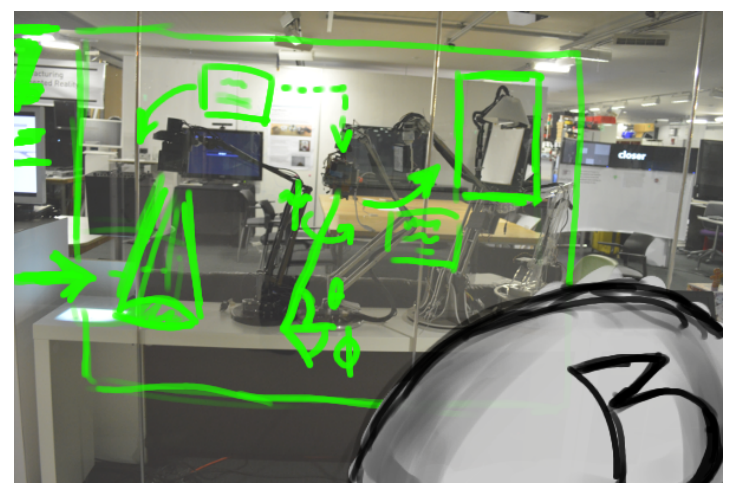

\subsection{Chapter Conclusion}

In this chapter we have presented further discussion around our approaches, as well as, results obtained in this work. Here, discussions related to the nature our approach is discussed in section 10.1. In section 10.2 we discussed about one of our strongest assumption taken in this work, and how it can be waved in new conditions. In section 10.3, we present relations of our approach with another type of information used in the literature that have 
strong similarities that is the light field theory (WANNER; GOLDLUECKE, 2012). In this discussion we also discuss ways to explore its potential in our work.

On the other hand, in section 10.4, we explore the possible usage of RF-RANSAC methods, proposed in this work, in other conditions. Combination of our work with other approaches and techniques is discussed in section 10.5. In this section, we focus in three methods that are combined with pose estimation in AR systems in several works: the classification, tracking and deformation model.

Sections 10.6 and 10.7 focus in more general usage of object based reality and the results obtained in this work applied for AR and interaction. In section 10.6, we discuss possibilities to extract other type of information for AR systems. Finally, in section 10.7 we focus in the usage of the markers in AR in order to create novel interaction methods. 


\section{Conclusion}

Augmented reality (AR) had become a widely developed idea from its beginning, with several ideas have been developed, such as (BENKO; WILSON; ZANNIER, 2014; KREVELEN; POELMAN, 2010). However, usage of real objects as markers to obtain the registering of virtual and real content is still a key factor in several applications of AR (KREVELEN; POELMAN, 2010) (also section 10.7). Those object can have different natures and behaviors such to be rigid (LEPETIT; MORENO-NOGUER; FUA, 2009), freely deformable (RENDL et al., 2014), foldable (STEIMLE; JORDT; MAES, 2013), articulated (PAUWELS; RUBIO; ROS, 2014). For each case, different methods for pose estimation where presented in the literature.

However, we observed that large part of those objects shares one common propriety; to be locally rigid (sections 3.4). By knowing how those locals change, it is possible to estimate those gamma of objects poses (as presented in chapter 5). In this work, we explore this idea as base for our contributions.

We presented novel approaches for pose estimation of different classes of objects, rigid and deformable. Unlike other work in literature, our approaches are based on the local pose estimation of feature points spread across the object surface (chapters 7 and 8). Those local poses can gives us rich information of the observed object pose.

Thus, our hypothesis here was that gathering more local information from each feature point, can give us information to estimate several types of objects poses.

In order to obtain those poses, we separated our work in three major parts: robust feature points extraction (chapter 6), local and global pose estimation based on RGB-D (chapter 7) and local and global pose estimation based on RGB (chapter 8).

First, we proposed a novel approach for background and affine transformation robust feature points, in order to be used as input to our local pose estimation. From section 9.1, we show that our approach achieved such characteristics, extracting feature points that can be observed in more different views, than, conventional methods of extraction.

Then, using those extracted feature points, we process the local poses based on RGB-D information (chapter 7). Those local poses are, then, used to find the global pose of the object, both rigid 7.3.1; and deformable 7.3.2. In section 9.2 we present our results based on the RGB-D cameras.

Those results illustrate that is possible obtain the pose of different kinds of objects using the same local pose information. We also present that it is possible to estimate objects poses, even with a single observation of the object (section 9.2.2). Making possible 
obtain registration for $\mathrm{AR}$, even in extreme cases that the object is barely visible.

On the other hand, we also present the pose estimation based on RGB information in chapter 8. In order to process the local poses in this condition, which lacks on depth information, we exploit machine learning methods.

Conventional approaches often are based on non-linear complex equation solving in order to estimate the minimal error of the projected points. Despite that, we replace this complex solver problem to an approach where local appearances changes of the feature points are trained using decision forests to find the local rotation (section 8.2).

By refining those local estimation regression returned by the forests, we obtain the full 6 DoF pose of the rigid object (section 8.4). Obtained results, section 9.3, show that our method generate comparable accuracy to state-of-art methods, with methods that conventionally has much lower computational demands (CRIMINISI; SHOTTON, 2013). We also present results of local rotations of deformable objects based on our machine learning approach, at section 9.3.3.

Although, both RGB and RGB-D results are not fully explored in real-time conditions, both approaches are based on methods that conventionally can generate fast results for real-time systems, such as RANSAC (FISCHLER; BOLLES, 1981) and regression forests (CRIMINISI; SHOTTON, 2013). Thus, it should be an easy incremental step to extend our results for true AR systems.

From those results (chapter 9), it is possible to confirm our hypothesis. Thus, showing that it is possible to estimate planar, rigid (sections 9.2.1 and 9.3.2) and deformable objects poses (sections 9.2.3 and 9.3.3) in both RGB and RGB-D capturing setups, by gathering local information and obtaining such local poses.

Several extensions and applications of our work are presented in chapter 10 as possibilities of future directions. Usage of our approaches as user interface (section 10.7) or creation of new types of markers (section 10.6) are possible direction that could be taken for creation of novel interfaces.

Extension of our method using other methods also is one possibility (sections 10.5). Once our approach is not a direct variation of existing methods, but instead, gather more information from local appearance, other top level methods could be extended by our local poses, such as tracking methods.

However, the most direct future work is to extend our method for real-time applications. In order to speed up the computational performance, the implementation in languages with better performance is one of the direct next step. Also, parallel processing approaches could be applied in order to obtain fast pose estimations.

Both, pose estimation based on RGB-D and RGB could be extended by parallel 
processing, once each feature point is processed separately for each other. Moreover, our RGB pose estimation approach is based on methods that could be easily implemented in parallel processing; the decision forest, per feature point pose and matrix multiplication for point transform.

Finally; this works just barely scratches the surface of an entire new idea for pose estimation. Local poses are not explored directly in several works, specially for different classes of object. Due to this novel nature, this work opens up new possibilities of object pose estimation in augmented reality, in order to create a tight link between reality and virtuality, as illustrated in figure 68 .

Figure 68 - Sobel filter (FAUGERAS, 1993) applied to the rendered image of our pose estimation result. The application of other concepts such as presenetd in (TOKUNAGA; NAKAMURA; TORI, 2009), can bring more results of object based AR.

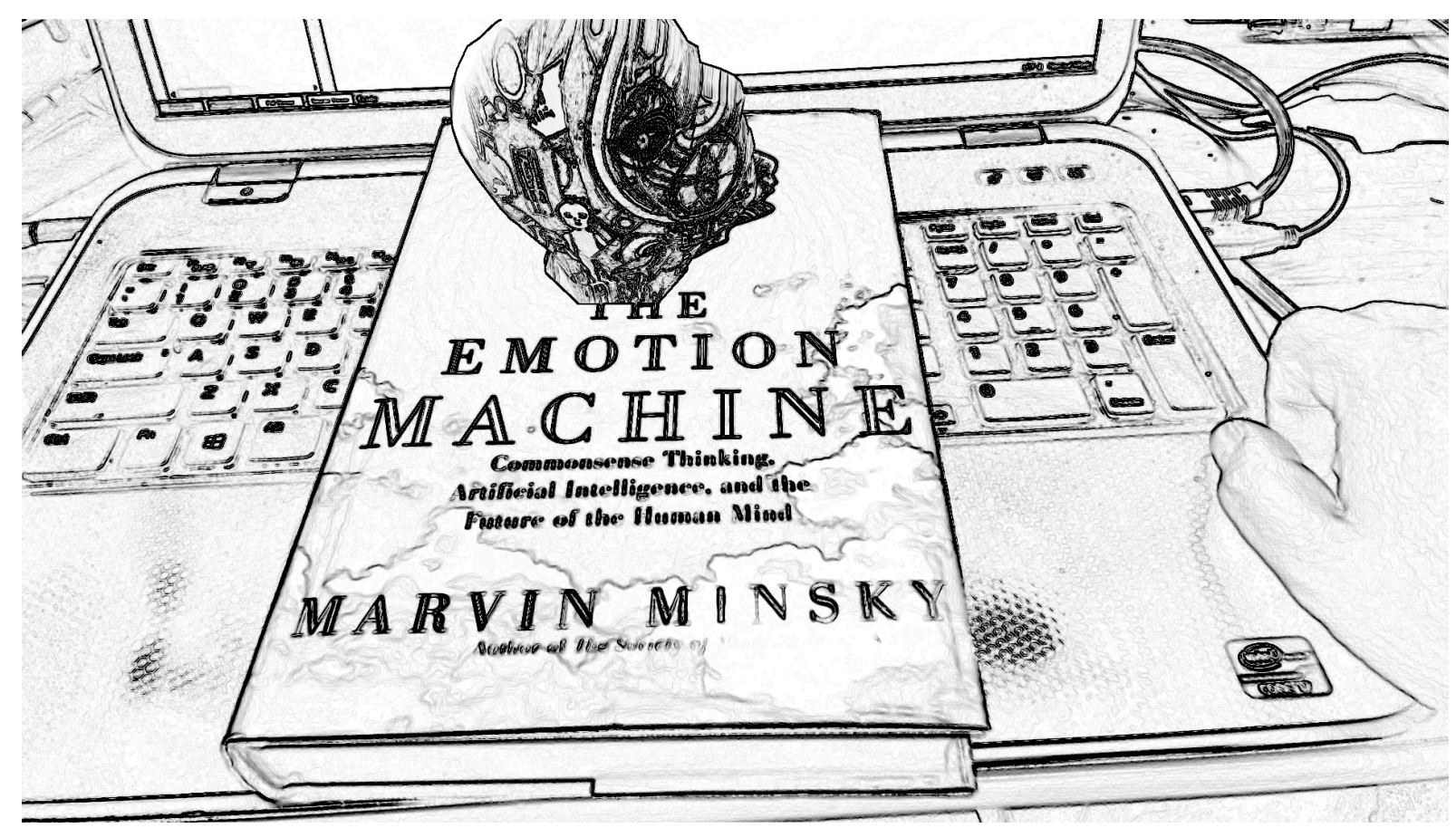

Source: Author. 


\section{Bibliography}

ABDEL-HAKIM, A.; FARAG, A. Csift: A sift descriptor with color invariant characteristics. In: Computer Vision and Pattern Recognition, 2006 IEEE Computer Society Conference on. [S.l.: s.n.], 2006. v. 2, p. 1978-1983. ISSN 1063-6919.

AKEnINE-MOLlER, T.; MOLlER, T.; HAINES, E. Real-Time Rendering. 3rd. ed. Natick, MA, USA: A. K. Peters, Ltd., 2008. ISBN 1439865299.

ALLEN, B.; CURLESS, B.; POPOVIć, Z. Articulated body deformation from range scan data. ACM Trans. Graph., ACM, New York, NY, USA, v. 21, n. 3, p. 612-619, jul. 2002. ISSN 0730-0301. Disponível em: <http://doi.acm.org/10.1145/566654.566626>.

ALLEN, B.; CURLESS, B.; POPOVIć, Z. The space of human body shapes: reconstruction and parameterization from range scans. ACM Trans. Graph., ACM, New York, NY, USA, v. 22, n. 3, p. 587-594, jul. 2003. ISSN 0730-0301. Disponível em: $<$ http://doi.acm.org/10.1145/882262.882311>.

ANGEL, E. Interactive Computer Graphics: A Top-down Approach Using OpenGL. Pearson/Addison-Wesley, 2006. (Computer graphics). ISBN 9780321312525. Disponível em: < https://books.google.com.br/books?id=Fsy $\backslash$ QgAACAAJ >.

ANGUELOV, D. et al. Scape: Shape completion and animation of people. In: $A C M$ SIGGRAPH 2005 Papers. New York, NY, USA: ACM, 2005. (SIGGRAPH '05), p. 408-416. Disponível em: <http://doi.acm.org/10.1145/1186822.1073207>.

AZUMA, R. T. A survey of augmented reality. Presence: Teleoperators and virtual environments, MIT Press, v. 6, n. 4, p. 355-385, 1997.

BAU, O. et al. Revel: Tactile feedback technology for augmented reality. In: ACM SIGGRAPH 2012 Emerging Technologies. New York, NY, USA: ACM, 2012. (SIGGRAPH '12), p. 17:1-17:1. ISBN 978-1-4503-1680-4. Disponível em: $<$ http://doi.acm.org/10.1145/2343456.2343473>.

BAUDISCH, P.; BECKER, T.; RUDECK, F. Lumino: Tangible blocks for tabletop computers based on glass fiber bundles. In: Proceedings of the SIGCHI Conference on Human Factors in Computing Systems. New York, NY, USA: ACM, 2010. (CHI '10), p. 1165-1174. ISBN 978-1-60558-929-9. Disponível em: <http://doi.acm.org/10.1145/1753326.1753500>.

BAY, H. et al. Speeded-up robust features (surf). Comput. Vis. Image Underst., Elsevier Science Inc., New York, NY, USA, v. 110, n. 3, p. 346-359, jun. 2008. ISSN 1077-3142. Disponível em: <http://dx.doi.org/10.1016/j.cviu.2007.09.014>.

BELLMORE, C.; PTUCHA, R.; SAVAKIS, A. Fusion of depth and color for an improved active shape model. In: Image Processing (ICIP), 2013 20th IEEE International Conference on. [S.1.: s.n.], 2013. p. 330-334.

BENKO, H.; WILSON, A. D.; ZANNIER, F. Dyadic projected spatial augmented reality. In: Proceedings of the 27th Annual ACM Symposium on User Interface Software 
and Technology. New York, NY, USA: ACM, 2014. (UIST '14), p. 645-655. ISBN 978-1-4503-3069-5. Disponível em: <http://doi.acm.org/10.1145/2642918.2647402>.

BESL, P.; MCKAY, N. D. A method for registration of 3-d shapes. Pattern Analysis and Machine Intelligence, IEEE Transactions on, v. 14, n. 2, p. 239-256, Feb 1992. ISSN 0162-8828.

BILLINGHURST, M.; KATO, H.; POUPYREV, I. The magicbook: a transitional ar interface. Computers and Graphics, v. 25, n. 5, p. 745-753, 2001. ISSN 0097-8493. Mixed realities - beyond conventions. Disponível em: <http://www.sciencedirect.com/science/ article/pii/S0097849301001170>.

BOSCH, A.; ZISSERMAN, A.; MUOZ, X. Image classification using random forests and ferns. In: Computer Vision, 200\%. ICCV 200\%. IEEE 11th International Conference on. [S.l.: s.n.], 2007. p. 1-8. ISSN 1550-5499.

BRACHMANN, E. et al. Learning 6d object pose estimation using 3d object coordinates. In: FLEET, D. et al. (Ed.). Computer Vision? ECCV 2014. [S.l.]: Springer International Publishing, 2014, (Lecture Notes in Computer Science, v. 8690). p. 536-551. ISBN 978-3-319-10604-5.

BROCKMEYER, E.; POUPYREV, I.; HUDSON, S. Papillon: Designing curved display surfaces with printed optics. In: Proceedings of the 26th Annual ACM Symposium on User Interface Software and Technology. New York, NY, USA: ACM, 2013. (UIST '13), p. 457-462. ISBN 978-1-4503-2268-3. Disponível em: $<$ http://doi.acm.org/10.1145/2501988.2502027>.

BUJNAK, M.; KUKELOVA, Z.; PAJDLA, T. A general solution to the p4p problem for camera with unknown focal length. In: Computer Vision and Pattern Recognition, 2008. CVPR 2008. IEEE Conference on. [S.l.: s.n.], 2008. p. 1-8. ISSN 1063-6919.

CANDOCIA, F. M.; MANDARINO, D. A semiparametric model for accurate camera response function modeling and exposure estimation from comparametric data. Image Processing, IEEE Transactions on, v. 14, n. 8, p. 1138-1150, Aug 2005. ISSN 1057-7149.

CHANG, W.; ZWICKER, M. Global registration of dynamic range scans for articulated model reconstruction. ACM Trans. Graph., ACM, New York, NY, USA, v. 30, n. 3, p. 26:1-26:15, maio 2011. ISSN 0730-0301. Disponível em: <http://doi.acm.org/10.1145/1966394.1966405>.

CHEN, S.; GINOSAR, R. Adaptive sensitivity ccd image sensor. In: Electrical and Electronics Engineers in Israel, 1995., Eighteenth Convention of. [S.l.: s.n.], 1995. p. 3.5.4/1-3.5.4/4.

CHIA, K. W.; CHEOK, A. D.; PRINCE, S. J. D. Online 6 dof augmented reality registration from natural features. In: Proceedings of the 1st International Symposium on Mixed and Augmented Reality. Washington, DC, USA: IEEE Computer Society, 2002. (ISMAR '02), p. 305-. ISBN 0-7695-1781-1. Disponível em: $<$ http://dl.acm.org/citation.cfm?id=850976.854957>.

CHOI, C.; CHRISTENSEN, H. 3d pose estimation of daily objects using an rgb-d camera. In: Intelligent Robots and Systems (IROS), 2012 IEEE/RSJ International Conference on. [S.l.: s.n.], 2012. p. 3342-3349. ISSN 2153-0858. 
CHOI, C.; CHRISTENSEN, H. Rgb-d object tracking: A particle filter approach on gpu. In: Intelligent Robots and Systems (IROS), 2013 IEEE/RSJ International Conference on. [S.l.: s.n.], 2013. p. 1084-1091. ISSN 2153-0858.

CHUM, O.; PAJDLA, T.; STURM, P. The geometric error for homographies. Comput. Vis. Image Underst., Elsevier Science Inc., New York, NY, USA, v. 97, n. 1, p. 86-102, jan. 2005. ISSN 1077-3142. Disponível em: <http://dx.doi.org/10.1016/j.cviu.2004.03.004>.

COMPORT, A. et al. Real-time markerless tracking for augmented reality: the virtual visual servoing framework. Visualization and Computer Graphics, IEEE Transactions on, v. 12 , n. 4, p. 615-628, July 2006. ISSN 1077-2626.

COSTA, M. S.; SHAPIRO, L. G. 3d object recognition and pose with relational indexing. Computer Vision and Image Understanding, v. 79, n. 3, p. $364-407,2000$. ISSN 1077-3142.

CRIMINISI, A.; SHOTTON, J. Decision Forests for Computer Vision and Medical Image Analysis. [S.1.]: Springer Publishing Company, Incorporated, 2013. ISBN 1447149289, 9781447149286.

CURHAN, J. R.; PENTLAND, A. Thin slices of negotiation: predicting outcomes from conversational dynamics within the first 5 minutes. Journal of Applied Psychology, American Psychological Association, v. 92, n. 3, p. 802, 2007.

DALAL, N.; TRIGGS, B. Histograms of oriented gradients for human detection. In: Proceedings of the 2005 IEEE Computer Society Conference on Computer Vision and Pattern Recognition (CVPR'05) - Volume 1 - Volume 01. Washington, DC, USA: IEEE Computer Society, 2005. (CVPR '05), p. 886-893. ISBN 0-7695-2372-2.

DAMEN, D. et al. Real-time learning and detection of 3d texture-less objects: A scalable approach. In: British Machine Vision Conference. [S.l.]: BMVA, 2012.

DAVISON, A. Real-time simultaneous localisation and mapping with a single camera. In: Computer Vision, 2003. Proceedings. Ninth IEEE International Conference on. [S.l.: s.n.], 2003. p. 1403-1410 vol.2.

DOSTAL, J.; KRISTENSSON, P. O.; QUIGLEY, A. Multi-view proxemics: Distance and position sensitive interaction. In: Proceedings of the 2Nd ACM International Symposium on Pervasive Displays. New York, NY, USA: ACM, 2013. (PerDis '13), p. 1-6. ISBN 978-1-4503-2096-2. Disponível em: <http://doi.acm.org/10.1145/2491568.2491570>.

EAGLE, N.; PENTLAND, A. S. Eigenbehaviors: Identifying structure in routine. Behavioral Ecology and Sociobiology, Springer, v. 63, n. 7, p. 1057-1066, 2009.

EDELMAN, S.; BULTHOFF, H. H. Orientation dependence in the recognition of familiar and novel views of three-dimensional objects. Vision Research, v. 32, n. 12, p. 2385 - 2400, 1992. ISSN 0042-6989. Disponível em: < http: //www.sciencedirect.com/science/article/pii/004269899290102O>.

FARAH, M. J. Is face recognition special? evidence from neuropsychology. Behavioural Brain Research, v. 76, n. 1-2, p. 181-189, 1996. ISSN 0166-4328. Advances in Understanding Visual Cortex Function. Disponível em: <http: //www.sciencedirect.com/science/article/pii/0166432895001980>. 
FAUGERAS, O. Three-dimensional Computer Vision: A Geometric Viewpoint. Cambridge, MA, USA: MIT Press, 1993. ISBN 0-262-06158-9.

FELZENSZWALB, P. et al. Object detection with discriminatively trained part-based models. Pattern Analysis and Machine Intelligence, IEEE Transactions on, v. 32, n. 9, p. 1627-1645, Sept 2010. ISSN 0162-8828.

FENZI, M. et al. Class generative models based on feature regression for pose estimation of object categories. In: Computer Vision and Pattern Recognition (CVPR), 2013 IEEE Conference on. [S.l.: s.n.], 2013. p. 755-762. ISSN 1063-6919.

FENZI, M. et al. Pose estimation of object categories in videos using linear programming. In: Applications of Computer Vision (WACV), 2015 IEEE Winter Conference on. [S.l.: s.n.], 2015. p. 821-828.

FISCHLER, M. A.; BOLLES, R. C. Random sample consensus: A paradigm for model fitting with applications to image analysis and automated cartography. Commun. ACM, ACM, New York, NY, USA, v. 24, n. 6, p. 381-395, jun. 1981. ISSN 0001-0782. Disponível em: < http://doi.acm.org/10.1145/358669.358692>.

FOLLMER, S. et al. inform: Dynamic physical affordances and constraints through shape and object actuation. In: Proceedings of the 26th Annual ACM Symposium on User Interface Software and Technology. New York, NY, USA: ACM, 2013. (UIST '13), p. 417-426. ISBN 978-1-4503-2268-3. Disponível em: <http://doi.acm.org/10.1145/2501988.2502032>.

FOSSUM, E. Cmos image sensors: electronic camera on a chip. In: Electron Devices Meeting, 1995. IEDM '95., International. [S.l.: s.n.], 1995. p. 17-25. ISSN 0163-1918.

GALASSO, F.; LASENBY, J. Shape from texture of developable surfaces via fourier analysis. In: Proceedings of the 3rd International Conference on Advances in Visual Computing - Volume Part I. Berlin, Heidelberg: Springer-Verlag, 2007. (ISVC'07), p. 702-713. ISBN 3-540-76857-2, 978-3-540-76857-9. Disponível em: $<$ http://dl.acm.org/citation.cfm?id=1779178.1779258>.

GAMAL, A. E.; ELTOUKHY, H. Cmos image sensors. Circuits and Devices Magazine, IEEE, v. 21, n. 3, p. 6-20, May 2005. ISSN 8755-3996.

GARDING, J. Shape from texture for smooth curved surfaces in perspective projection. Journal of Mathematical Imaging and Vision, Kluwer Academic Publishers, v. 2, n. 4, p. 327-350, 1992. ISSN 0924-9907.

GARRO, V.; CROSILLA, F.; FUSIELLO, A. Solving the pnp problem with anisotropic orthogonal procrustes analysis. In: 3D Imaging, Modeling, Processing, Visualization and Transmission (3DIMPVT), 2012 Second International Conference on. [S.l.: s.n.], 2012. p. 262-269.

GAUTHIER, I. et al. Expertise for cars and birds recruits brain areas involved in face recognition. Nature neuroscience, Nature Publishing Group, v. 3, n. 2, p. 191-197, 2000.

GRAFAREND, E. W.; SHAN, J. Closed-form solution of p4p or the three-dimensional resection problem in terms of möbius barycentric coordinates. Journal of Geodesy, Springer-Verlag, v. 71, n. 4, p. 217-231, 1997. ISSN 0949-7714. Disponível em: <http://dx.doi.org/10.1007/s001900050089>. 
GRASSET, R.; DüNSER, A.; BILLINGHURST, M. Edutainment with a mixed reality book: A visually augmented illustrative childrens' book. In: Proceedings of the 2008 International Conference on Advances in Computer Entertainment Technology. New York, NY, USA: ACM, 2008. (ACE '08), p. 292-295. ISBN 978-1-60558-393-8. Disponível em: <http://doi.acm.org/10.1145/1501750.1501819>.

GU, C.; REN, X. Discriminative mixture-of-templates for viewpoint classification. In: Proceedings of the 11th European Conference on Computer Vision: Part V. Berlin, Heidelberg: Springer-Verlag, 2010. (ECCV'10), p. 408-421. ISBN 3-642-15554-5, 978-3-642-15554-3.

GUPTA, S. et al. Learning rich features from rgb-d images for object detection and segmentation. Springer International Publishing, v. 8695, p. 345-360, 2014.

HAGBI, N. et al. In-place augmented reality. In: Proceedings of the 7th IEEE/ACM International Symposium on Mixed and Augmented Reality. Washington, DC, USA: IEEE Computer Society, 2008. (ISMAR '08), p. 135-138. ISBN 978-1-4244-2840-3. Disponível em: <http://dx.doi.org/10.1109/ISMAR.2008.4637339>.

HAIN, R.; KAHLER, C.; TROPEA, C. Comparison of ccd, cmos and intensified cameras. Experiments in Fluids, Springer-Verlag, v. 42, n. 3, p. 403-411, 2007. ISSN 0723-4864. Disponível em: <http://dx.doi.org/10.1007/s00348-006-0247-1>.

HARA, K.; CHELLAPPA, R. Growing regression forests by classification: Applications to object pose estimation. In: FLEET, D. et al. (Ed.). Computer Vision ? ECCV 2014. [S.1.]: Springer International Publishing, 2014, (Lecture Notes in Computer Science, v. 8690). p. 552-567. ISBN 978-3-319-10604-5.

HE, K.; SIGAL, L.; SCLAROFF, S. Parameterizing object detectors in the continuous pose space. In: FLEET, D. et al. (Ed.). Computer Vision? ECCV 2014. [S.l.]: Springer International Publishing, 2014, (Lecture Notes in Computer Science, v. 8692). p. 450-465. ISBN 978-3-319-10592-5.

HEIKKILA, J.; SILVEN, O. A four-step camera calibration procedure with implicit image correction. In: Proceedings of the 1997 Conference on Computer Vision and Pattern Recognition (CVPR '97). Washington, DC, USA: IEEE Computer Society, 1997. (CVPR '97), p. 1106-. ISBN 0-8186-7822-4. Disponível em: $<$ http://dl.acm.org/citation.cfm?id=794189.794489>.

HENRY, P. et al. Rgb-d mapping: Using kinect-style depth cameras for dense 3d modeling of indoor environments. Int. J. Rob. Res., Sage Publications, Inc., Thousand Oaks, CA, USA, v. 31, n. 5, p. 647-663, abr. 2012. ISSN 0278-3649. Disponível em: <http://dx.doi.org/10.1177/0278364911434148>.

HERTZMANN, A.; SEITZ, S. Example-based photometric stereo: shape reconstruction with general, varying brdfs. Pattern Analysis and Machine Intelligence, IEEE Transactions on, v. 27, n. 8, p. 1254-1264, Aug 2005. ISSN 0162-8828.

HEUN, V.; HOBIN, J.; MAES, P. Reality editor: Programming smarter objects. In: Proceedings of the 2013 ACM Conference on Pervasive and Ubiquitous Computing Adjunct Publication. New York, NY, USA: ACM, 2013. (UbiComp '13 Adjunct), p. 307-310. ISBN 978-1-4503-2215-7. Disponível em: <http://doi.acm.org/10.1145/2494091.2494185>. 
HINSKE, S.; LANGHEINRICH, M.; LAMPE, M. Towards guidelines for designing augmented toy environments. In: Proceedings of the rth ACM Conference on Designing Interactive Systems. New York, NY, USA: ACM, 2008. (DIS '08), p. 78-87. ISBN 978-1-60558-002-9. Disponível em: <http://doi.acm.org/10.1145/1394445.1394454>.

HOLLER, J. et al. Chapter $2-\{\mathrm{M} 2 \mathrm{M}\}$ to iot - the vision. In: HOLLER, J. et al. (Ed.). From Machine-To-Machine to the Internet of Things. Oxford: Academic Press, 2014. p. 9 - 37. ISBN 978-0-12-407684-6. Disponível em: <http://www.sciencedirect.com/science/article/pii/B9780124076846000024>.

HOLROYD, M. et al. A photometric approach for estimating normals and tangents. ACM Trans. Graph., ACM, New York, NY, USA, v. 27, n. 5, p. 133:1-133:9, dez. 2008. ISSN 0730-0301. Disponível em: <http://doi.acm.org/10.1145/1409060.1409086>.

HOLZER, S. et al. Distance transform templates for object detection and pose estimation. In: Computer Vision and Pattern Recognition, 2009. CVPR 2009. IEEE Conference on. [S.1.: s.n.], 2009. p. 1177-1184. ISSN 1063-6919.

HORAUD, R. et al. An analytic solution for the perspective 4-point problem. In: Computer Vision and Pattern Recognition, 1989. Proceedings CVPR '89., IEEE Computer Society Conference on. [S.l.: s.n.], 1989. p. 500-507. ISSN 1063-6919.

HORN, B. K. P. Closed-form solution of absolute orientation using unit quaternions. Journal of the Optical Society of America A, v. 4, n. 4, p. 629-642, 1987.

HSU, E. et al. Light mixture estimation for spatially varying white balance. ACM Trans. Graph., ACM, New York, NY, USA, v. 27, n. 3, p. 70:1-70:7, aug 2008. ISSN 0730-0301. Disponível em: <http://doi.acm.org/10.1145/1360612.1360669>.

HU, Z. Y.; WU, F. C. A note on the number of solutions of the noncoplanar p4p problem. IEEE Trans. Pattern Anal. Mach. Intell., IEEE Computer Society, Washington, DC, USA, v. 24, n. 4, p. 550-555, abr. 2002. ISSN 0162-8828. Disponível em: $<$ http://dx.doi.org/10.1109/34.993561>.

ISARD, M.; BLAKE, A. Condensation conditional density propagation for visual tracking. International Journal of Computer Vision, Kluwer Academic Publishers, v. 29, n. 1, p. 5-28, 1998. ISSN 0920-5691.

ISHII, H. et al. Radical atoms: Beyond tangible bits, toward transformable materials. interactions, ACM, New York, NY, USA, v. 19, n. 1, p. 38-51, jan 2012. ISSN 1072-5520. Disponível em: <http://doi.acm.org/10.1145/2065327.2065337>.

ISHII, H.; MAZALEK, A.; LEE, J. Bottles as a minimal interface to access digital information. In: CHI '01 Extended Abstracts on Human Factors in Computing Systems. New York, NY, USA: ACM, 2001. (CHI EA '01), p. 187-188. ISBN 1-58113-340-5. Disponível em: <http://doi.acm.org/10.1145/634067.634180>.

ISHII, H.; ULLMER, B. Tangible bits: Towards seamless interfaces between people, bits and atoms. In: Proceedings of the ACM SIGCHI Conference on Human Factors in Computing Systems. New York, NY, USA: ACM, 1997. (CHI '97), p. 234-241. ISBN 0-89791-802-9. Disponível em: <http://doi.acm.org/10.1145/258549.258715>. 
IZADI, S. et al. Kinectfusion: Real-time 3d reconstruction and interaction using a moving depth camera. In: Proceedings of the 24th Annual ACM Symposium on User Interface Software and Technology. New York, NY, USA: ACM, 2011. (UIST '11), p. 559-568. ISBN 978-1-4503-0716-1. Disponível em: < http://doi.acm.org/10.1145/2047196.2047270>.

JACHNIK, J.; NEWCOMBE, R. A.; DAVISON, A. J. Real-time surface light-field capture for augmentation of planar specular surfaces. In: Proceedings of the 2012 IEEE International Symposium on Mixed and Augmented Reality (ISMAR). Washington, DC, USA: IEEE Computer Society, 2012. (ISMAR '12), p. 91-97. ISBN 978-1-4673-4660-3. Disponível em: <http://dx.doi.org/10.1109/ISMAR.2012.6402544>.

JOLLIFFE, I. Principal component analysis. [S.l.]: Wiley Online Library, 2002.

JORDT, A.; KOCH, R. Direct model-based tracking of 3d object deformations in depth and color video. Int. J. Comput. Vision, Kluwer Academic Publishers, Hingham, MA, USA, v. 102, n. 1-3, p. 239-255, mar. 2013. ISSN 0920-5691. Disponível em: $<$ http://dx.doi.org/10.1007/s11263-012-0572-1>.

KABSCH, W. A solution for the best rotation to relate two sets of vectors. Acta Crystallographica Section A, v. 32, n. 5, p. 922-923, Sep 1976. Disponível em: <http://dx.doi.org/10.1107/S0567739476001873>.

KALAL, Z.; MATAS, J.; MIKOLAJCZYK, K. P-n learning: Bootstrapping binary classifiers by structural constraints. In: Computer Vision and Pattern Recognition (CVPR), 2010 IEEE Conference on. [S.l.: s.n.], 2010. p. 49-56. ISSN 1063-6919.

KALMAN, R. E. A new approach to linear filtering and prediction problems. ASME Journal of Basic Engineering, 1960.

KE, Y.; SUKTHANKAR, R. Pca-sift: a more distinctive representation for local image descriptors. In: Computer Vision and Pattern Recognition, 2004. CVPR 2004. Proceedings of the 2004 IEEE Computer Society Conference on. [S.l.: s.n.], 2004. v. 2, p. II-506-II-513 Vol.2. ISSN 1063-6919.

KIM, C. et al. Scene reconstruction from high spatio-angular resolution light fields. $A C M$ Trans. Graph., ACM, New York, NY, USA, v. 32, n. 4, p. 73:1-73:12, jul. 2013. ISSN 0730-0301. Disponível em: <http://doi.acm.org/10.1145/2461912.2461926>.

KIPNIS, A.; SHAMIR, A. Cryptanalysis of the hfe public key cryptosystem by relinearization. In: WIENER, M. (Ed.). Advances in Cryptology ? CRYPTO? 99. [S.1.]: Springer Berlin Heidelberg, 1999, (Lecture Notes in Computer Science, v. 1666). p. 19-30. ISBN 978-3-540-66347-8.

KLEIN, G.; MURRAY, D. Parallel tracking and mapping for small AR workspaces. In: Proc. Sixth IEEE and ACM International Symposium on Mixed and Augmented Reality (ISMAR'07). Nara, Japan: [s.n.], 2007.

KREVELEN, D. W. F. van; POELMAN, R. A Survey of Augmented Reality Technologies, Applications and Limitations. The International Journal of Virtual Reality, v. 9, n. 2, p. 1-20, jun. 2010. 
KŕIVáNEK, J.; FERWERDA, J. A.; BALA, K. Effects of global illumination approximations on material appearance. ACM Trans. Graph., ACM, New York, NY, USA, v. 29, n. 4, p. 112:1-112:10, jul 2010. ISSN 0730-0301. Disponível em: $<$ http://doi.acm.org/10.1145/1778765.1778849>.

KRULL, A. et al. 6-dof model based tracking via object coordinate regression. In: CREMERS, D. et al. (Ed.). Computer Vision - ACCV 2014. [S.1.]: Springer International Publishing, 2015, (Lecture Notes in Computer Science, v. 9006). p. 384-399. ISBN 978-3-319-16816-6.

KUKELOVA, Z.; BUJNAK, M.; PAJDLA, T. Automatic generator of minimal problem solvers. In: FORSYTH, D.; TORR, P.; ZISSERMAN, A. (Ed.). Computer Vision ECCV 2008. [S.l.]: Springer Berlin Heidelberg, 2008, (Lecture Notes in Computer Science, v. 5304). p. 302-315. ISBN 978-3-540-88689-1.

LAI, K. et al. Sparse distance learning for object recognition combining rgb and depth information. In: Robotics and Automation (ICRA), 2011 IEEE International Conference on. [S.l.: s.n.], 2011. p. 4007-4013. ISSN 1050-4729.

LEE, J. C.; HUDSON, S. E.; TSE, E. Foldable interactive displays. In: Proceedings of the 21st Annual ACM Symposium on User Interface Software and Technology. New York, NY, USA: ACM, 2008. (UIST '08), p. 287-290. ISBN 978-1-59593-975-3. Disponível em: $<$ http://doi.acm.org/10.1145/1449715.1449763>.

LEE, K.-R.; NGUYEN, T. Robust tracking and mapping with a handheld rgb-d camera. In: Applications of Computer Vision (WACV), 2014 IEEE Winter Conference on. [S.1.: s.n.], 2014. p. 1120-1127.

LEE, W.; PARK, N.; WOO, W. Depth-assisted real-time 3d object detection for augmented reality. In: International Conference on Artificial Reality and Telexistence (ICAT'11). [S.l.: s.n.], 2011.

LEITHINGER, D. et al. Physical telepresence: Shape capture and display for embodied, computer-mediated remote collaboration. In: Proceedings of the 27th Annual ACM Symposium on User Interface Software and Technology. New York, NY, USA: ACM, 2014. (UIST '14), p. 461-470. ISBN 978-1-4503-3069-5. Disponível em: <http://doi.acm.org/10.1145/2642918.2647377>.

LEPETIT, V.; LAGGER, P.; FUA, P. Randomized trees for real-time keypoint recognition. In: Computer Vision and Pattern Recognition, 2005. CVPR 2005. IEEE Computer Society Conference on. [S.l.: s.n.], 2005. v. 2, p. 775-781 vol. 2. ISSN 1063-6919.

LEPETIT, V.; MORENO-NOGUER, F.; FUA, P. Epnp: An accurate o(n) solution to the pnp problem. International Journal of Computer Vision, Springer US, v. 81, n. 2, p. 155-166, 2009. ISSN 0920-5691. Disponível em: <http://dx.doi.org/10.1007/s11263-008-0152-6>.

LI, H. et al. Robust single-view geometry and motion reconstruction. ACM Transactions on Graphics (Proceedings SIGGRAPH Asia 2009), ACM, v. 28, n. 5, December 2009.

LI, H.; SUMNER, R. W.; PAULY, M. Global correspondence optimization for non-rigid registration of depth scans. Computer Graphics Forum (Proc. SGP'08), Eurographics Association, ETH Zurich, v. 27, n. 5, July 2008. 
LI, S.; XU, C. A stable direct solution of perspective-three-point problem. International Journal of Pattern Recognition and Artificial Intelligence, v. 25, n. 05, p. 627-642, 2011. Disponível em: <http://www.worldscientific.com/doi/abs/10.1142/S0218001411008774>.

LI, S.; XU, C.; XIE, M. A robust o(n) solution to the perspective-n-point problem. Pattern Analysis and Machine Intelligence, IEEE Transactions on, v. 34, n. 7, p. 1444-1450, July 2012. ISSN 0162-8828.

LIMA, J. et al. Texture-less planar object detection and pose estimation using depth-assisted rectification of contours. In: Mixed and Augmented Reality (ISMAR), 2012 IEEE International Symposium on. [S.l.: s.n.], 2012. p. 297-298.

LIMA, J. P. et al. Model based markerless 3d tracking applied to augmented reality. Journal on 3D Interactive Systems, v. 1, 2010.

LIN, D. et al. Deep lac: Deep localization, alignment and classification for fine-grained recognition. In: The IEEE Conference on Computer Vision and Pattern Recognition (CVPR). [S.l.: s.n.], 2015.

LIU, C. et al. Automatic estimation and removal of noise from a single image. Pattern Analysis and Machine Intelligence, IEEE Transactions on, v. 30, n. 2, p. 299-314, Feb 2008. ISSN 0162-8828.

LO, J.; PAULOS, E. Shrinkycircuits: Sketching, shrinking, and formgiving for electronic circuits. In: Proceedings of the 27th Annual ACM Symposium on User Interface Software and Technology. New York, NY, USA: ACM, 2014. (UIST '14), p. 291-299. ISBN 978-1-4503-3069-5. Disponível em: <http://doi.acm.org/10.1145/2642918.2647421>.

LOBAY, A.; FORSYTH, D. A. Shape from texture without boundaries. Int. J. Comput. Vision, Kluwer Academic Publishers, Hingham, MA, USA, v. 67, n. 1, p. 71-91, abr. 2006. ISSN 0920-5691. Disponível em: <http://dx.doi.org/10.1007/s11263-006-4068-8>.

LOESCH, A. et al. Generic edgelet-based tracking of 3d objects in real-time. In: 2015 IEEE/RSJ International Conference on Intelligent Robots and Systems. [S.1.]: IEEE, 2015.

LOWE, D. Fitting parameterized three-dimensional models to images. Pattern Analysis and Machine Intelligence, IEEE Transactions on, v. 13, n. 5, p. 441-450, May 1991. ISSN 0162-8828.

LOWE, D. Object recognition from local scale-invariant features. In: Computer Vision, 1999. The Proceedings of the Seventh IEEE International Conference on. [S.l.: s.n.], 1999. v. 2, p. 1150-1157 vol.2.

LOWE, D. G. Distinctive image features from scale-invariant keypoints. Int. J. Comput. Vision, Kluwer Academic Publishers, Hingham, MA, USA, v. 60, n. 2, p. 91-110, nov. 2004. ISSN 0920-5691. Disponível em: <http://dx.doi.org/10.1023/B: VISI.0000029664.99615.94>.

LU, C.-P.; HAGER, G. D.; MJOLSNESS, E. Fast and globally convergent pose estimation from video images. IEEE Trans. Pattern Anal. Mach. Intell., IEEE Computer Society, Washington, DC, USA, v. 22, n. 6, p. 610-622, jun. 2000. ISSN 0162-8828. Disponível em: <http://dx.doi.org/10.1109/34.862199>. 
MAGNENAT, S. et al. Live texturing of augmented reality characters from colored drawings. Visualization and Computer Graphics, IEEE Transactions on, v. 21, n. 11, p. 1201-1210, Nov 2015. ISSN 1077-2626.

MALIK, J.; ROSENHOLTZ, R. Computing local surface orientation and shape from texture for curved surfaces. International Journal of Computer Vision, Kluwer Academic Publishers, v. 23, n. 2, p. 149-168, 1997. ISSN 0920-5691.

MALIK, S.; MCDONALD, C.; ROTH, G. Hand tracking for interactive pattern-based augmented reality. In: Mixed and Augmented Reality, 2002. ISMAR 2002. Proceedings. International Symposium on. [S.l.: s.n.], 2002. p. 117-126.

MARCON, M. et al. 3d correspondences in textured depth-maps through planar similarity transform. In: Emerging Signal Processing Applications (ESPA), 2012 IEEE International Conference on. [S.l.: s.n.], 2012. p. 17-20.

MARSHALL, G.; STUTZ, G. Handbook of Optical and Laser Scanning, Second Edition. Taylor \& Francis, 2011. (Optical Science and Engineering). ISBN 9781439808795.

Disponível em: <https://books.google.com.br/books?id=MLWUatLv0s0C > .

MARWAH, K. et al. Compressive Light Field Photography using Overcomplete Dictionaries and Optimized Projections. ACM Trans. Graph. (Proc. SIGGRAPH), ACM, New York, NY, USA, v. 32, n. 4, p. 1-11, 2013.

MASSARWI, F.; GOTSMAN, C.; ELBER, G. Papercraft models using generalized cylinders. In: Computer Graphics and Applications, 200\%. PG '0\%. 15th Pacific Conference on. [S.l.: s.n.], 2007. p. 148-157. ISSN 1550-4085.

MATAS, J. et al. Robust wide-baseline stereo from maximally stable extremal regions. Image and vision computing, Elsevier, v. 22, n. 10, p. 761-767, 2004.

MATUSIK, W. et al. Image-based visual hulls. In: Proceedings of the 27th Annual Conference on Computer Graphics and Interactive Techniques. New York, NY, USA: ACM Press/Addison-Wesley Publishing Co., 2000. (SIGGRAPH '00), p. 369-374. ISBN 1-58113-208-5. Disponível em: <http://dx.doi.org/10.1145/344779.344951>.

MAZALEK, A.; NITSCHE, M. Tangible interfaces for real-time 3d virtual environments. In: Proceedings of the International Conference on Advances in Computer Entertainment Technology. New York, NY, USA: ACM, 2007. (ACE '07), p. 155-162. ISBN 978-1-59593-640-0. Disponível em: <http://doi.acm.org/10.1145/1255047.1255080>.

MEI, L. et al. Robust object pose estimation via statistical manifold modeling. In: Computer Vision (ICCV), 2011 IEEE International Conference on. [S.1.: s.n.], 2011. p. 967-974. ISSN 1550-5499.

MICHEL, F. et al. Pose estimation of kinematic chain instances via object coordinate regression. In: XIE, M. W. J. X.; TAM, G. K. L. (Ed.). Proceedings of the British Machine Vision Conference (BMVC). BMVA Press, 2015. p. 181.1-181.11. ISBN 1-901725-53-7. Disponível em: <https://dx.doi.org/10.5244/C.29.181>.

MIKOLAJCZYK, K.; SCHMID, C. A performance evaluation of local descriptors. IEEE Transactions on Pattern Analysis \& Machine Intelligence, v. 27, n. 10, p. 1615-1630, 2005. Disponível em: <http://lear.inrialpes.fr/pubs/2005/MS05>. 
MITANI, J.; SUZUKI, H. Making papercraft toys from meshes using stripbased approximate unfolding. ACM Trans. Graph., ACM, New York, NY, USA, v. 23, n. 3, p. 259-263, aug 2004. ISSN 0730-0301. Disponível em: <http: //doi.acm.org/10.1145/1015706.1015711>.

MIYASHITA, L. et al. 3d motion sensing of any object without prior knowledge. ACM Trans. Graph., ACM, New York, NY, USA, v. 34, n. 6, p. 218:1-218:11, out. 2015. ISSN 0730-0301. Disponível em: <http://doi.acm.org/10.1145/2816795.2818133>.

MOREL, J.-M.; YU, G. Asift: A new framework for fully affine invariant image comparison. SIAM J. Img. Sci., Society for Industrial and Applied Mathematics, Philadelphia, PA, USA, v. 2, n. 2, p. 438-469, apr 2009. ISSN 1936-4954. Disponível em: $<$ http://dx.doi.org/10.1137/080732730>.

MURASE, H.; NAYAR, S. K. Parametric eigenspace representation for visual learning and recognition. In: . [s.n.], 1993. v. 2031, p. 378-391. Disponível em: <http://dx.doi.org/10.1117/12.146641>.

MURPHY, K. P. Machine Learning: A Probabilistic Perspective. [S.l.]: The MIT Press, 2012. ISBN 0262018020, 9780262018029.

MUSÉ, P. et al. Unsupervised thresholds for shape matching. In: IEEE. Image Processing, 2003. ICIP 2003. Proceedings. 2003 International Conference on. [S.l.], 2003. v. 2, p. II-647.

NEWCOMBE, R. A. et al. Kinectfusion: Real-time dense surface mapping and tracking. In: Proceedings of the 2011 10th IEEE International Symposium on Mixed and Augmented Reality. Washington, DC, USA: IEEE Computer Society, 2011. (ISMAR '11), p. 127-136. ISBN 978-1-4577-2183-0. Disponível em: <http://dx.doi.org/10.1109/ISMAR.2011.6092378>.

NIIYAMA, R. et al. Sticky actuator: Free-form planar actuators for animated objects. In: Proceedings of the Ninth International Conference on Tangible, Embedded, and Embodied Interaction. New York, NY, USA: ACM, 2015. (TEI '15), p. 77-84. ISBN 978-1-4503-3305-4. Disponível em: <http://doi.acm.org/10.1145/2677199.2680600>.

OLBERDING, S.; WESSELY, M.; STEIMLE, J. Printscreen: Fabricating highly customizable thin-film touch-displays. In: Proceedings of the 27th Annual ACM Symposium on User Interface Software and Technology. New York, NY, USA: ACM, 2014. (UIST '14), p. 281-290. ISBN 978-1-4503-3069-5. Disponível em: $<$ http://doi.acm.org/10.1145/2642918.2647413>.

PAPAZOV, C.; BURSCHKA, D. An efficient ransac for 3d object recognition in noisy and occluded scenes. In: Proceedings of the 10th Asian Conference on Computer Vision - Volume Part I. Berlin, Heidelberg: Springer-Verlag, 2011. (ACCV'10), p. 135-148. ISBN 978-3642-19314-9. Disponível em: <http://dl.acm.org/citation.cfm?id=1964320.1964334>.

PARK, Y.; LEPETIT, V.; WOO, W. Texture-less object tracking with online training using an rgb-d camera. In: Mixed and Augmented Reality (ISMAR), 2011 10th IEEE International Symposium on. [S.l.: s.n.], 2011. p. 121-126. 
PAULUS, C. et al. Augmented reality during cutting and tearing of deformable objects. In: Mixed and Augmented Reality (ISMAR), 2015 IEEE International Symposium on. [S.l.: s.n.], 2015. p. 54-59.

PAUWELS, K.; RUBIO, L.; ROS, E. Real-time model-based articulated object pose detection and tracking with variable rigidity constraints. In: Computer Vision and Pattern Recognition (CVPR), 2014 IEEE Conference on. [S.l.: s.n.], 2014. p. 3994-4001.

PEDERSEN, E. W.; HORNBAEK, K. mixitui: A tangible sequencer for electronic live performances. In: Proceedings of the 3rd International Conference on Tangible and Embedded Interaction. New York, NY, USA: ACM, 2009. (TEI '09), p. 223-230. ISBN 978-1-60558-493-5. Disponível em: <http://doi.acm.org/10.1145/1517664.1517713>.

PEDERSEN, E. W.; HORNBÆK, K. Tangible bots: Interaction with active tangibles in tabletop interfaces. In: Proceedings of the SIGCHI Conference on Human Factors in Computing Systems. New York, NY, USA: ACM, 2011. (CHI '11), p. 2975-2984. ISBN 978-1-4503-0228-9. Disponível em: <http://doi.acm.org/10.1145/1978942.1979384>.

PEKELNY, Y.; GOTSMAN, C. Articulated object reconstruction and markerless motion capture from depth video. Computer Graphics Forum, Blackwell Publishing Ltd, v. 27, n. 2, p. 399-408, 2008. ISSN 1467-8659. Disponível em: <http: //dx.doi.org/10.1111/j.1467-8659.2008.01137.x>.

PEPIK, B. et al. 3d2pm ? 3d deformable part models. In: FITZGIBBON, A. et al. (Ed.). Computer Vision? ECCV 2012. [S.1.]: Springer Berlin Heidelberg, 2012, (Lecture Notes in Computer Science, v. 7577). p. 356-370. ISBN 978-3-642-33782-6.

PILET, J.; LEPETIT, V.; FUA, P. Fast non-rigid surface detection, registration and realistic augmentation. Int. J. Comput. Vision, Kluwer Academic Publishers, Hingham, MA, USA, v. 76, n. 2, p. 109-122, fev. 2008. ISSN 0920-5691. Disponível em: <http://dx.doi.org/10.1007/s11263-006-0017-9>.

POUPYREV, I.; NASHIDA, T.; OKABE, M. Actuation and tangible user interfaces: The vaucanson duck, robots, and shape displays. In: Proceedings of the 1st International Conference on Tangible and Embedded Interaction. New York, NY, USA: ACM, 2007. (TEI '07), p. 205-212. ISBN 978-1-59593-619-6. Disponível em: <http://doi.acm.org/10.1145/1226969.1227012>.

POUPYREV, I.; NEWTON-DUNN, H.; BAU, O. D20: Interaction with multifaceted display devices. In: CHI '06 Extended Abstracts on Human Factors in Computing Systems. New York, NY, USA: ACM, 2006. (CHI EA '06), p. 1241-1246. ISBN 1-59593-298-4. Disponível em: <http://doi.acm.org/10.1145/1125451.1125683>.

PRESS, W. H. et al. Numerical Recipes 3rd Edition: The Art of Scientific Computing. 3. ed. New York, NY, USA: Cambridge University Press, 2007. ISBN 0521880688, 9780521880688 .

PRINCE, S. J. D.; XU, K.; CHEOK, A. D. Augmented reality camera tracking with homographies. IEEE Comput. Graph. Appl., IEEE Computer Society Press, Los Alamitos, CA, USA, v. 22, n. 6, p. 39-45, nov. 2002. ISSN 0272-1716. Disponível em: <http://dx.doi.org/10.1109/MCG.2002.1046627>. 
PRITCHARD, D.; HEIDRICH, W. Cloth motion capture. Computer Graphics Forum, Blackwell Publishing, Inc, v. 22, n. 3, p. 263-271, 2003. ISSN 1467-8659. Disponível em: $<$ http://dx.doi.org/10.1111/1467-8659.00673>.

REES, E. L. Graphical discussion of the roots of a quartic equation. In: Advances in Cryptology? CRYPTO? 99. Mathematical Association of America, 1922, (The American Mathematical Monthly, v. 29). p. 51-55. Disponível em: <http: //www.jstor.org/stable/2972804>.

RENDL, C. et al. Flexsense: A transparent self-sensing deformable surface. In: Proceedings of the 27th Annual ACM Symposium on User Interface Software and Technology. New York, NY, USA: ACM, 2014. (UIST '14), p. 129-138. ISBN 978-1-4503-3069-5. Disponível em: <http://doi.acm.org/10.1145/2642918.2647405>.

SAKOE, H.; CHIBA, S. Dynamic programming algorithm optimization for spoken word recognition. Acoustics, Speech and Signal Processing, IEEE Transactions on, v. 26, n. 1, p. 43-49, Feb 1978. ISSN 0096-3518.

SALVI, J.; PAGES, J.; BATLLE, J. Pattern codification strategies in structured light systems. Pattern recognition, Elsevier, v. 37, n. 4, p. 827-849, 2004.

SATO, M.; POUPYREV, I.; HARRISON, C. Touché: Enhancing touch interaction on humans, screens, liquids, and everyday objects. In: Proceedings of the SIGCHI Conference on Human Factors in Computing Systems. New York, NY, USA: ACM, 2012. (CHI '12), p. 483-492. ISBN 978-1-4503-1015-4. Disponível em: <http://doi.acm.org/10.1145/2207676.2207743>.

SCHIETTECATTE, B.; VANDERDONCKT, J. Audiocubes: A distributed cube tangible interface based on interaction range for sound design. In: Proceedings of the 2Nd International Conference on Tangible and Embedded Interaction. New York, NY, USA: ACM, 2008. (TEI '08), p. 3-10. ISBN 978-1-60558-004-3. Disponível em: $<$ http://doi.acm.org/10.1145/1347390.1347394>.

SCHULMAN, J. et al. Tracking deformable objects with point clouds. In: Robotics and Automation (ICRA), 2013 IEEE International Conference on. [S.l.: s.n.], 2013. p. 1130-1137. ISSN 1050-4729.

SHARP, T. Implementing decision trees and forests on a gpu. In: FORSYTH, D.; TORR, P.; ZISSERMAN, A. (Ed.). Computer Vision ? ECCV 2008. [S.l.]: Springer Berlin Heidelberg, 2008, (Lecture Notes in Computer Science, v. 5305). p. 595-608. ISBN 978-3-540-88692-1.

SHIMIZU, S. et al. Object pose estimation using category information from a single image. In: Frontiers of Computer Vision (FCV), 2015 21st Korea-Japan Joint Workshop on. [S.l.: s.n.], 2015. p. 1-4.

SHOTTON, J. et al. Real-time human pose recognition in parts from single depth images. In: Computer Vision and Pattern Recognition (CVPR), 2011 IEEE Conference on. [S.l.: s.n.], 2011. p. 1297-1304. ISSN 1063-6919.

SHOTTON, J. et al. Scene coordinate regression forests for camera relocalization in rgb-d images. In: Proceedings of the 2013 IEEE Conference on Computer Vision and 
Pattern Recognition. Washington, DC, USA: IEEE Computer Society, 2013. (CVPR '13), p. 2930-2937. ISBN 978-0-7695-4989-7.

SOCHER, R. et al. Convolutional-recursive deep learning for 3d object classification. In: PEREIRA, F. et al. (Ed.). Advances in Neural Information Processing Systems 25. Curran Associates, Inc., 2012. p. 656-664. Disponível em: < http://papers.nips.cc/paper/ 4773-convolutional-recursive-deep-learning-for-3d-object-classification.pdf $>$.

STEFANOU, S.; ARGYROS, A. Efficient scale and rotation invariant object detection based on hogs and evolutionary optimization techniques. In: BEBIS, G. et al. (Ed.). Advances in Visual Computing. [S.l.]: Springer Berlin Heidelberg, 2012, (Lecture Notes in Computer Science, v. 7431). p. 220-229. ISBN 978-3-642-33178-7.

STEIMLE, J.; JORDT, A.; MAES, P. Flexpad: Highly flexible bending interactions for projected handheld displays. In: Proceedings of the SIGCHI Conference on Human Factors in Computing Systems. New York, NY, USA: ACM, 2013. (CHI '13), p. 237-246. ISBN 978-1-4503-1899-0. Disponível em: <http://doi.acm.org/10.1145/2470654.2470688>.

STURM, P.; MAYBANK, S. On plane-based camera calibration: A general algorithm, singularities, applications. In: Computer Vision and Pattern Recognition, 1999. IEEE Computer Society Conference on. [S.l.: s.n.], 1999. v. 1, p. -437 Vol. 1. ISSN 1063-6919.

SUGANO, N.; KATO, H.; TACHIBANA, K. The effects of shadow representation of virtual objects in augmented reality. In: Mixed and Augmented Reality, 2003. Proceedings. The Second IEEE and ACM International Symposium on. [S.l.: s.n.], 2003. p. 76-83.

SUMNER, R. W.; SCHMID, J.; PAULY, M. Embedded deformation for shape manipulation. ACM Trans. Graph., ACM, New York, NY, USA, v. 26, n. 3, jul. 2007. ISSN 0730-0301. Disponível em: <http://doi.acm.org/10.1145/1276377.1276478>.

TAGUCHI, Y. et al. Point-plane slam for hand-held 3d sensors. In: Robotics and Automation (ICRA), 2013 IEEE International Conference on. [S.l.: s.n.], 2013. p. 5182-5189. ISSN 1050-4729.

TEJANI, A. et al. Latent-class hough forests for 3d object detection and pose estimation. In: FLEET, D. et al. (Ed.). Computer Vision? ECCV 2014. [S.1.]: Springer International Publishing, 2014, (Lecture Notes in Computer Science, v. 8694). p. 462-477. ISBN 978-3-319-10598-7.

TODD, J. T. The visual perception of 3d shape. Trends in Cognitive Sciences, v. 8, n. 3, p. 115 - 121, 2004. ISSN 1364-6613. Disponível em: < http://www.sciencedirect.com/ science/article/pii/S1364661304000233>.

TOKUNAGA, D. M. et al. Local 3d pose estimation of feature points based on RGB-D information for object based augmented reality. In: Virtual, Augmented and Mixed Reality - 7th International Conference, VAMR 2015, Held as Part of HCI International 2015, Los Angeles, CA, USA, August 2-7, 2015, Proceedings. [S.1.: s.n.], 2015. p. 130-141.

TOKUNAGA, D. M.; NAKAMURA, R.; TORI, R. Non-photorealistic 3d video-avatar. In: SIGGRAPH '09: Posters. New York, NY, USA: ACM, 2009. (SIGGRAPH '09), p. 101:1-101:1. Disponível em: <http://doi.acm.org/10.1145/1599301.1599402>. 
TORKI, M.; ELGAMMAL, A. Regression from local features for viewpoint and pose estimation. In: Proceedings of the 2011 International Conference on Computer Vision. Washington, DC, USA: IEEE Computer Society, 2011. (ICCV '11), p. 2603-2610. ISBN 978-1-4577-1101-5. Disponível em: <http://dx.doi.org/10.1109/ICCV.2011.6126549>.

TRUCCO, E.; VERRI, A. Introductory Techniques for 3-D Computer Vision. Upper Saddle River, NJ, USA: Prentice Hall PTR, 1998. ISBN 0132611082.

TUNWATTANAPONG, B. et al. Acquiring reflectance and shape from continuous spherical harmonic illumination. ACM Trans. Graph., ACM, New York, NY, USA, v. 32, n. 4, p. 109:1-109:12, jul. 2013. ISSN 0730-0301. Disponível em: <http://doi.acm.org/10.1145/2461912.2461944>.

ULLMER, B. et al. Tangible menus and interaction trays: Core tangibles for common physical/digital activities. In: Proceedings of the $2 N d$ International Conference on Tangible and Embedded Interaction. New York, NY, USA: ACM, 2008. (TEI '08), p. 209-212. ISBN 978-1-60558-004-3. Disponível em: <http://doi.acm.org/10.1145/1347390.1347436>.

VACCHETTI, L.; LEPETIT, V.; FUA, P. Combining edge and texture information for real-time accurate $3 \mathrm{~d}$ camera tracking. In: Proceedings of the 3rd IEEE/ACM International Symposium on Mixed and Augmented Reality. Washington, DC, USA: IEEE Computer Society, 2004. (ISMAR '04), p. 48-57. ISBN 0-7695-2191-6. Disponível em: <http://dx.doi.org/10.1109/ISMAR.2004.24>.

VEERARAGHAVAN, A. et al. Dappled photography: Mask enhanced cameras for heterodyned light fields and coded aperture refocusing. ACM Trans. Graph., ACM, New York, NY, USA, v. 26, n. 3, jul. 2007. ISSN 0730-0301. Disponível em: $<$ http://doi.acm.org/10.1145/1276377.1276463>.

VIOLA, P.; JONES, M. J. Robust real-time face detection. International journal of computer vision, Springer, v. 57, n. 2, p. 137-154, 2004.

WANG, J. et al. Locality-constrained linear coding for image classification. In: Computer Vision and Pattern Recognition (CVPR), 2010 IEEE Conference on. [S.l.: s.n.], 2010. p. 3360-3367. ISSN 1063-6919.

WANNER, S.; GOLDLUECKE, B. Globally consistent depth labeling of 4d light fields. In: Computer Vision and Pattern Recognition (CVPR), 2012 IEEE Conference on. [S.l.: s.n.], 2012. p. 41-48. ISSN 1063-6919.

WATT, A. 3D Computer Graphics. 2nd. ed. Boston, MA, USA: Addison-Wesley Longman Publishing Co., Inc., 1993. ISBN 0201631865.

WERNECK, N. L.; COSTA, A. H. R. Corisco: Robust edgel-based orientation estimation for generic camera models. Image and Vision Computing, v. 31, n. 12, p. 969 - 981, 2013. ISSN 0262-8856. Disponível em: < http://www.sciencedirect.com/science/article/pii/ S0262885613001534>.

WESIERSKI, D.; HORAIN, P. Pose-configurable generic tracking of elongated objects. In: Computer Vision (ICCV), 2013 IEEE International Conference on. [S.l.: s.n.], 2013. p. 2920-2927. ISSN 1550-5499. 
WHELAN, T. et al. Deformation-based loop closure for large scale dense rgb-d slam. In: Intelligent Robots and Systems (IROS), 2013 IEEE/RSJ International Conference on. [S.l.: s.n.], 2013. p. 548-555. ISSN 2153-0858.

WIETHOFF, A. et al. Sketch-a-tui: Low cost prototyping of tangible interactions using cardboard and conductive ink. In: Proceedings of the Sixth International Conference on Tangible, Embedded and Embodied Interaction. New York, NY, USA: ACM, 2012. (TEI '12), p. 309-312. ISBN 978-1-4503-1174-8. Disponível em: <http://doi.acm.org/10.1145/2148131.2148196>.

WU, Y.; HU, Z. Pnp problem revisited. Journal of Mathematical Imaging and Vision, Kluwer Academic Publishers, v. 24, n. 1, p. 131-141, 2006. ISSN 0924-9907. Disponível em: <http://dx.doi.org/10.1007/s10851-005-3617-z>.

XIAO, X.; TOME, B.; ISHII, H. Andante: Walking figures on the piano keyboard to visualize musical motion. In: Proceedings of the International Conference on New Interfaces for Musical Expression. London, United Kingdom: [s.n.], 2014. p. 629-632.

XU, C.; PRINCE, J. Snakes, shapes, and gradient vector flow. Image Processing, IEEE Transactions on, v. 7, n. 3, p. 359-369, Mar 1998. ISSN 1057-7149.

YAMASHITA, J. et al. Tangible avatar and tangible earth: A novel interface for astronomy education. In: CHI 'O'7 Extended Abstracts on Human Factors in Computing Systems. New York, NY, USA: ACM, 2007. (CHI EA '07), p. 2777-2782. ISBN 978-1-59593-642-4. Disponível em: <http://doi.acm.org/10.1145/1240866.1241078>.

YILMAZ, A.; JAVED, O.; SHAH, M. Object tracking: A survey. ACM Comput. Surv., ACM, New York, NY, USA, v. 38, n. 4, dez. 2006. ISSN 0360-0300. Disponível em: $<$ http://doi.acm.org/10.1145/1177352.1177355>.

YUAN, L. et al. Image deblurring with blurred/noisy image pairs. ACM Trans. Graph., ACM, New York, NY, USA, v. 26, n. 3, jul 2007. ISSN 0730-0301. Disponível em: $<$ http://doi.acm.org/10.1145/1276377.1276379>.

ZHANG, H. et al. Vision-based pose estimation for space objects by gaussian process regression. In: Aerospace Conference, 2015 IEEE. [S.l.: s.n.], 2015. p. 1-9.

ZHANG, Z. A flexible new technique for camera calibration. IEEE Trans. Pattern Anal. Mach. Intell., IEEE Computer Society, Washington, DC, USA, v. 22, n. 11, p. 1330-1334, nov 2000. ISSN 0162-8828. Disponível em: <http://dx.doi.org/10.1109/34.888718>.

ZHEN, X. et al. Supervised descriptor learning for multi-output regression. In: Computer Vision and Pattern Recognition (CVPR), 2015 IEEE Conference on. [S.1.: s.n.], 2015. p. 1211-1218.

ZHENG, Y. et al. Revisiting the pnp problem: A fast, general and optimal solution. In: Computer Vision (ICCV), 2013 IEEE International Conference on. [S.l.: s.n.], 2013. p. 2344-2351. ISSN 1550-5499. 